Portland State University

PDXScholar

$12-4-2020$

\title{
Entrainment Processes for a Jet in Cross-flow: the Quantification of Turbulent Contributions and their Importance on Accurate Modeling
}

Graham Asher Freedland

Portland State University

Follow this and additional works at: https://pdxscholar.library.pdx.edu/open_access_etds

Part of the Aerodynamics and Fluid Mechanics Commons, and the Mechanical Engineering Commons Let us know how access to this document benefits you.

Recommended Citation

Freedland, Graham Asher, "Entrainment Processes for a Jet in Cross-flow: the Quantification of Turbulent Contributions and their Importance on Accurate Modeling" (2020). Dissertations and Theses. Paper 5618. https://doi.org/10.15760/etd.7490

This Dissertation is brought to you for free and open access. It has been accepted for inclusion in Dissertations and Theses by an authorized administrator of PDXScholar. Please contact us if we can make this document more accessible: pdxscholar@pdx.edu. 
Entrainment Processes for a Jet in Cross-flow: The Quantification of Turbulent Contributions and their Importance on Accurate Modeling

by

Graham Asher Freedland

A dissertation submitted in fulfillment of the requirements for the degree of

Doctor of Philosophy

in

Mechanical Engineering

\author{
Dissertation Committee: \\ Raúl Bayoán Cal, Chair \\ Gerald Recktenwald \\ Mark Weislogel \\ Stephen Solovitz \\ Dacian Daescu
}

Portland State University 


\begin{abstract}
A jet in cross flow (JICF) is examined experimentally by injecting a stream of air into crossing fluid with an aim into quantifying entrainment process and downstream evolution. The behavior of JICF is important to fields ranging from turbine-blade cooling to smokestack pollution and volcanic eruption dynamics. Existing simplified volcanic plume models are tested; most importantly, the near-field contributions of complex interconnected vortex systems, which present significant uncertainties because they assume negligible turbulence. While jets in irrotational cross-flow have been investigated, this analysis has focused on the interaction between a turbulent jet in low and highly turbulent cross-flow created by an active grid. Instantaneous velocity fields were collected over seven planes using particle image velocimetry (PIV). A center-plane $(x-y)$ and six planes parallel to the floor $(x-z)$ highlight the interaction and resulting vortex systems. Various jet-to-cross-flow velocity ratios, $R_{v}$, were collected for each plane, which allow for computation of mean statistics and Reynolds stresses. Analysis was focused in five stages: a) identification of differences in the development of the jet across various inflow conditions, b) analysis of the vortex systems through transport and critical points analysis, c) decomposition of the flow structures to identify and remove the highest-order contributions to turbulence kinetic energy and d) extraction of reduced order modeling closure terms and e) optimization of closure terms for the simplified one-dimensional model, Plumeria. These five stages provided a comprehensive description of the role of cross-flow turbulence on the development of JICF. Noteworthy findings include
\end{abstract}


significant changes in wake recovery and the near-wake recirculation region that impacted near-field entrainment; increased entrainment for high cross-flow turbulence after the collapse of the potential core due to increased engulfment and viscous nibbling between turbulent fluids; the presence of shear layer and wake vortices through critical point analysis; and the absence of entrainment and shear layer expansion near the exit. Most importantly, the negligible entrainment near the exit and impact of small scale turbulent features that must be included for any specific model to yield reasonable predictions is highlighted. By laying the foundation for a more nuanced approach to JICF, it is possible to more precisely summarize the complex features observed in this work through simplified descriptions that can be of benefit to both engineering design and geophysical modelling. 


\section{Acknowledgements}

This work would not be possible without the incredible support I have received over the last six years. I would first like to acknowledge my advisor, Dr. Raúl Bayoán Cal, who I am indebted to for his incredible support - both in taking a chance in giving me this opportunity and nurturing my curiosity throughout my graduate education. Special thanks to Dr. Stephen Solovitz, and Dr. Larry Mastin for their encouragement and discussions that helped shape the interdisciplinary research in this dissertation.

This work would also not be possible without the support of the graduate students of the Wind Energy and Turbulence Lab. Their assistance in the laboratory, discussions on theory and fundamentals and moral support at conferences helped guide my research. Special thanks to the former students who taught me how to devise and run experiments, and my committee, who tirelessly reviewed my research for my benefit and treated me as a colleague at the conclusion of my defense.

I would like to thank Portland State University's Mechanical and Materials Engineering department for their support, and the National Science Foundation for their funding through NSF grant NSF-EAR-1346580.

And of course, I must also thank the friends and family who supported me through every stage of this process. To my parents, this is not only my accomplish- 
ment, it is a recognition of the hard work and dedication you taught me. To my incredible partner, I could not have done any of this without you. Thank you for literally dragging me across the finish line. This work is dedicated to you. 


\section{Table of Contents}

Abstract

Acknowledgements

List of Tables $\quad$ ix

List of Figures $\quad$ X

$\begin{array}{lll}\text { Chapter } 1 & \text { Introduction } & 1\end{array}$

1.1 Turbulent Entrainment . . . . . . . . . . . . . . . 3

1.1.1 Vortex Systems . . . . . . . . . . . . . 5

1.2 Turbulence Modelling . . . . . . . . . . . . . . . . . 8

1.2.1 Low Order Modelling . . . . . . . . . . . . . . . . . 10

1.3 Creation of Experimental Turbulence . . . . . . . . . . . . . . 12

$\begin{array}{lll}\text { Chapter } 2 & \text { Theory and Methodology } & 16\end{array}$

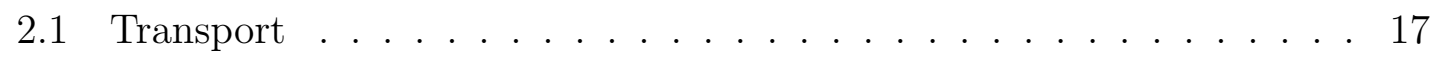

2.2 Reduced Order Entrainment Models . . . . . . . . . . . . . 21

2.3 Large Eddy Simulation . . . . . . . . . . . . . . . . . 23

2.3.1 Sub-Grid Scale Two-Equation Models . . . . . . . . . . . . . 28 
2.4 Dissipation from Modified Structure Function Method . . . . . . . . . 29

2.5 Critical Point Identification and Classification . . . . . . . . . . . . 33

2.5.1 Vortex Classification . . . . . . . . . . . . . 36

2.6 Proper Orthogonal Decomposition . . . . . . . . . . . . . . . . 40

2.7 Auto-correlation of Coherent Structures . . . . . . . . . . . . . . . . 43

$\begin{array}{lll}\text { Chapter } 3 & \text { Experimental Setup } & 44\end{array}$

3.1 Facilities . . . . . . . . . . . . . . . . . . . 44

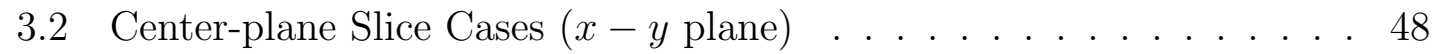

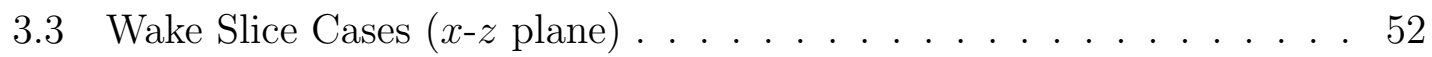

3.4 Variable Density Plume . . . . . . . . . . . . . . . . . . 54

$\begin{array}{lll}\text { Chapter } 4 & \text { Results } & 56\end{array}$

4.1 Center-plane $(x-y) \quad \ldots \ldots \ldots \ldots$

4.1.1 Mean Flow Statistics and Reynolds Stresses . . . . . . . . . . 57

4.1.2 Scaling and Characterization . . . . . . . . . . . . . 64

4.1.3 Shear Layer Identification . . . . . . . . . . . . . . . . 69

4.1.4 Transport Analysis . . . . . . . . . . . . . . . . 76

4.1.5 Summary .................... . . 84

4.2 Jet Slices $(x-z) \ldots \ldots \ldots 6$

4.2.1 Transport Analysis . . . . . . . . . . . . . . . 92

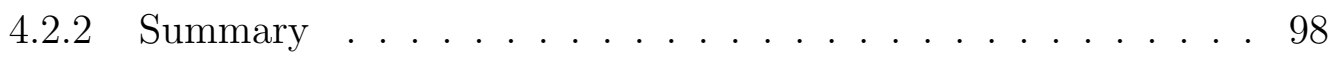

4.3 Proper Orthogonal Decomposition . . . . . . . . . . . . . . . . 99

4.3.1 POD Modes . . . . . . . . . . . . . . 100

4.3.2 Select Mode Reconstructions . . . . . . . . . . . . . . . . 107 
4.3 .3 Summary . . . . . . . . . . . . . . . . . . . . 108

4.4 Reduced Order Modelling Parameters . . . . . . . . . . . . . . . . 109

4.4.1 Eddy Viscosity . . . . . . . . . . . . . . . . . . . . . . . 109

4.4.2 Dissipation (Modified Structure Function Method) _ . . . . 115

4.4 .3 Kolmogorov Length Scales . . . . . . . . . . . . . . . . . 123

4.4.4 Modelling Closure Terms . . . . . . . . . . . . . . . . . . . . 124

4.4 .5 Summary . . . . . . . . . . . . . . . . . . 127

4.5 Critical Point Identification and Classification _ . . . . . . . . 128

4.5.1 Centerplane $(x-y) \ldots \ldots \ldots$

4.5 .2 Wake Vortices . . . . . . . . . . . . . . . . . . . . 132

4.5 .3 Summary . . . . . . . . . . . . . . . . . . . 135

4.6 Autocorrelation of Coherent Structures . . . . . . . . . . . 136

4.6.1 Coherent Structure Measurements . . . . . . . . . . . . . 136

4.6.2 Comparison of Coherent Structures . . . . . . . . . . . 140

4.6 .3 Summary . . . . . . . . . . . . . . . . 146

4.7 Low Order Model Investigation _ . . . . . . . . . . . . . 147

4.7.1 Direct Calculation of Closure Terms . . . . . . . . . . . . . 151

4.7 .2 Extracted Closure Terms . . . . . . . . . . . . . . . 156

4.7 .3 Summary . . . . . . . . . . . . . . . . 157

$\begin{array}{lll}\text { Chapter } 5 & \text { Conclusions } & 159\end{array}$

$\begin{array}{lll}\text { Chapter } 6 & \text { Future Work } & 164\end{array}$

6.1 Low Order Model Comparisons . . . . . . . . . . . . . . . . 164

6.2 Vortex System Entrainment Contributions . . . . . . . . . . 167 
6.3 Buoyant Plumes . . . . . . . . . . . . . . . . . . . . . . 169

References

Appendix A Shear Layer Identification 185

Appendix B Relative Axis Transformations 


\section{List of Tables}

Table 3.1 Experimental data for the jet including the inflow velocity, $u_{\infty}$, the jet exit velocity, $v_{\text {jet }}$, Jet-to-cross-flow velocity ratios, $R_{v}=$ $v_{\text {jet }} / u_{\infty}$, and computed far-field location, $\xi_{*}^{F F}=0.3 R_{v}^{2}$. Jet velocity was kept constant $\left(v_{\text {jet }}=26.0 \mathrm{~m} / \mathrm{s}\right)$ and the length scale was normalized relative to the jet diameter. . . . . . . . . 49

Table 3.2 Experimental data for the inflow including cross-flow velocity, $u_{\infty}$, turbulence intensity, $u_{r m s}^{\prime} / \bar{U}$, integral length scale, $L_{x} / D$, the turbulent Reynolds number, $\operatorname{Re}_{L_{x}}=u_{r m s}^{\prime} L_{x} / \nu$, and the inflow Reynolds number, $\operatorname{Re}_{D}=u_{\infty} D / \nu$ used in the current work. The length scale was normalized relative to the jet di-

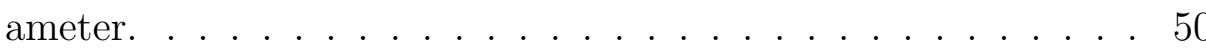

Table 3.3 Reynolds number of the inflow, $\operatorname{Re}_{\infty}=u_{\infty} D / \nu$, for each case. . 54

Table 3.4 Experimental pilot data for a variable density jet including the inflow velocity, $u_{\infty}$, the jet exit velocity, $v_{\text {jet }}$, jet-to-cross-flow velocity ratios, $R_{v}=v_{\text {jet }} / u_{\infty}$, and computed far-field location, $\xi_{*}^{F F}=0.3 R_{v}^{2}$. Jet velocity was kept constant $\left(v_{\text {jet }}=10.0\right.$ and $22.0 \mathrm{~m} / \mathrm{s}$ for Argon and Helium respectively) and the length scale was normalized by the jet diameter. . . . . . . . . . . 55 


\section{List of Figures}

Figure 1.1 Volcanic plume spread after the 1980 Mt. St. Helens eruption from Holasek \& Self $[1$, p. 8474]. . . . . . . . . . . . . . . . . 2

Figure 1.2 Sketch of the Turbulent/Non-turbulent interface and entrainment processes from Bisset et al. [2]. . . . . . . . . . . . .

Figure 1.3 Cartoon schematic of the four dominant flow features for a jet in cross-flow from Fric \& Roshko [3, p. 2]. . . . . . . . . . 6

Figure 1.4 Approaches to simplified models for a jet in cross-flow from Coelho \& Hunt [4, p. 96]. Modelling the jet as a) an entraining surface, and b) as a pair of vortices removed complexity by summarizing turbulent contributions through transport and aerodynamic principles. . . . . . . . . . . . . . 11

Figure 1.5 Volcanic plume data used for validation of volcanic plume models from Mastin et al. [5]. Using physical features of an eruption as inputs allowed comparison to converge on optimal configurations. Due to significant uncertainty, incorrect computations could have resulted in an order of magnitude

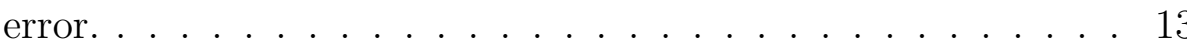

Figure 2.1 Transform of a bending jet from cartesian to relative coordinates. 20 
Figure 2.2 Dissipation results for data obtained using synthetic PIV data from Xu \& Chen [6, fig. 7]. The empirical fit (from (2.53)) yields the coefficients $\alpha$ and $\beta(i=1,2)$. . . . . . . 32

Figure 2.3 Classification of critical points projected onto the $p q$ domain from Perry \& Chong [7, fig. 2]. . . . . . . . . . . . . . 39

Figure 3.1 Schematic of PSU Wind Tunnel Test Section (not to scale). . . 46 Figure 3.2 Schematic of jet system supported by frame (not shown) to hold level and flush with the test section floor. All pipe diameters refer to inner diameter measurement. . . . . . . . . . . . 47

Figure 3.3 Process of identifying the center of the jet using a) raw PIV $\bar{V}$ data (before post-processing to reverse $x$ direction), and b) the slope of the velocity profile. This produces c), the normalized profile on a centered $x$-axis. . . . . . . . . . . . . . . 48

Figure 3.4 Velocity profile and turbulence intensity for two passive and two active grid cases. . . . . . . . . . . . . . . . 51

Figure 3.5 Schematic of SPIV windows parallel to the floor (not to scale). Each slice was spaced $\Delta y / D=1$ from $y / D=1 \rightarrow 6$ and extended from $\sim 5 D$ upstream to $\sim 18 D$ downstream of the jet exit. .................... 53

Figure 4.1 The streamwise $\bar{U}_{*}(\mathrm{a}-\mathrm{b})$, vertical $\bar{V}_{*}(\mathrm{c}-\mathrm{d})$, and out-of-plane $\bar{W}_{*}$ (e-f) mean flow statistics for one passive grid and one

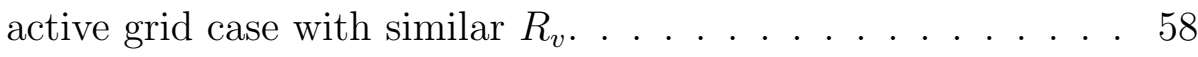


Figure 4.2 Components of Reynolds stress tensor including the streamwise (a-b) and vertical (c-d), Reynolds normal stresses, and the Reynolds shear stress (e-f) for one passive grid and one active grid case with similar $R_{v}$. . . . . . . . . . . 63

Figure 4.3 Centerline trajectories for each case, evaluated as the largest $\bar{V}$ at each height and fitted with a power law function. Near/farfield transition point $\left(\xi_{*}^{F F}=0.3 R_{v}^{2}\right)$ has been marked for each centerline $(\times)$, but exists past $x_{*}=10$ for high $R_{v}$. . . . . 65

Figure 4.4 Power law coefficients for the centerline fit, $Y=A X^{b}$ where $X$ and $Y$ are scaled by $D(\mathrm{a}, \mathrm{d}), R_{v} D(\mathrm{~b}, \mathrm{e})$, and $R_{v}^{2} D(\mathrm{c}, \mathrm{f})$. Resulting converged data fits were included for $A$. . . . . . . 66

Figure 4.5 Velocity (a, b, and c) and Reynolds stress (d, e and f) components on a relative axis along the centerline of the jet. . . . 68

Figure 4.6 Reynolds normal stress component $\overline{u^{\prime} u^{\prime}}$ along the centerline of the jet for all a) passive and b) active grid cases. The peak location (coinciding with the convergence of the normal stress peaks, marked with $\times$ ) was found to move closer to the exit as $R_{v}$ decreased. . . . . . . . . . . . . . . 70

Figure 4.7 Evaluation of the scaled location of the peak radial normal stress, $\xi_{*}^{0}$. The locations were normalized by $R_{v}^{2} D$ and fitted to highlight the relationship to $R_{v}$. . . . . . . . . . 71 
Figure 4.8 Profiles of the a) turbulence intensity, $I$, b) the squared Reynolds shear stress, $\left\langle u^{\prime} v^{\prime}\right\rangle_{r}^{2}$, and c) the squared vorticity profiles, $\left\langle\partial_{r} \bar{V}_{r}-\partial_{\xi} \bar{U}_{r}\right\rangle^{2}$ at three lengths of the jet for PG (solid) and AG (dashed). . . . . . . . . . . . . . . 72

Figure 4.9 Comparison of the JCB definition from the squared shear stress profile $\left\langle u^{\prime} v^{\prime}\right\rangle_{r}^{2}$ and the squared vorticity $\left\langle\partial_{r} \bar{V}_{r}-\partial_{\xi} \bar{U}_{r}\right\rangle^{2}$. The shear stress profile allowed for definition farther downstream. . . . . . . . . . . . . . . . . . . 73

Figure 4.10 The jet cross-flow boundary at the outer edge of the shear layer for PG (solid) and AG (dashed). Three representative cases (with similar $R_{v}$ ) for PG and AG are shown. . . . . . . . 74

Figure 4.11 The width of the jet as a function of the scaled jet length, $\xi / \xi^{0}$ for all center-plane cases. . . . . . . . . . . . . . . . . . 75

Figure 4.12 Integrated momentum and energy transport terms from Chapter 2.1 evaluated across the width of the jet for two similar $R_{v} .77$

Figure 4.13 Integrated transport terms from Chapter 2.1 along the leading edge and lee-side JCB with the normal directed into the jet (positive for entrainment). . . . . . . . . . . 78

Figure 4.14 Turbulence energy production, $\mathcal{P}$, averaged across the width

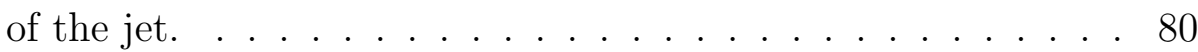

Figure 4.15 Momentum and energy transport of the inflow approaching the jet from $y_{*}=0 \rightarrow \xi^{0}$ for all cases. . . . . . . . . 81

Figure 4.16 Momentum $\Delta M / M_{\infty}$ and energy $\Delta E / E_{\infty}$ transport deficit from upstream of the jet $\left(M_{\infty}\right)$ to lee-side wake region. . . . 82 
Figure 4.17 Mean flow statistics for $x-z$ slice PIV planes at the height $y_{*}=4$ for stream-wise, vertical, and out-of-plane velocity components $\left(\bar{U}_{*}, \bar{V}_{*}\right.$, and $\bar{W}_{*}$ respectively). . . . . . . . 8

Figure 4.18 Method of defining the edges of the jet in the $x-z$ plane for $R_{v}=14, y_{*}=5$. For each case (panel a), a series of lines were extended from the center of the jet, allowing a fraction of unresolved data points to be recorded. The edge of the jet was defined as the width at half maximum of a Gaussian fit. This was repeated for b) $\theta=0 \rightarrow 2 \pi$ to provide the radius of the jet, $r_{j}(\theta) \ldots \ldots \ldots \ldots \ldots$

Figure 4.19 Three-dimensional jet volume construction (a, b) using the method in Figure 4.18 on each $x-z$ plane $(\mathrm{c}, \mathrm{d})$ for a low and high cross-flow velocity case. . . . . . . . . . . . . 90

Figure 4.20 Area of the jet conic sections at each height and the integrated

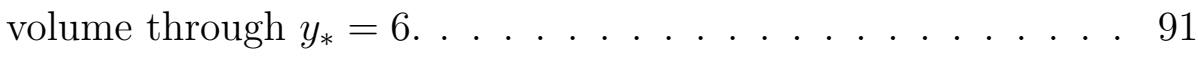

Figure 4.21 Net momentum and energy transport integrated along the closed area defined by the JCB. The positive normal was defined into the jet to denote entrainment with positive values. . 92

Figure 4.22 The upstream location, $x_{*}^{a}$, and downstream location, $x_{*}^{b}$, used to evaluate the momentum and transport deficit within the wake. 94

Figure 4.23 The wake momentum deficit, $\Delta U / U$, for each $x$-z plane plotted as a function of $x-x_{J C B} . \ldots . . . . . . . . .995$ 
Figure 4.24 Momentum and energy transport deficit, $\Delta M / M_{\infty}$ (first and third column) and $\Delta E / E_{\infty}$ (second and fourth column) respectively, comparing the inflow and downstream wake starting from the lee-side JCB. Terms were integrated from $z_{*}=-6$ to 6 to encompass both inside and out of the wake. . . . . . 96

Figure 4.25 Cumulative (percent) energy per mode for each inflow condition as a result of POD on the relative fluctuating flow-fields. . 100

Figure 4.26 Two-dimensional POD modes for velocity fluctuations normal to the centerline, $u_{r}^{\prime}$, for $R_{v} \approx 6$. The leading edge and lee-side JCB (dashed) and $\xi^{0}$ (o-o) were plotted to spatially relate key locations of energy. . . . . . . . . . . . . . . 102

Figure 4.27 Two-dimensional POD modes for velocity fluctuations tangential to the centerline, $v_{r}^{\prime}$, for $R_{v} \approx 6$. The leading edge and lee-side JCB (dashed) and $\xi^{0}$ (o-o) were plotted to spatially relate key locations of energy. . . . . . . . . . . . . 104

Figure 4.28 Two-dimensional POD modes for out-of-plane velocity fluctuations, $w_{r}^{\prime}$, for $R_{v} \approx 6$. The leading edge and lee-side JCB (dashed) and $\xi^{0}$ (o-o) were plotted to spatially relate key locations of energy. . . . . . . . . . . . . . 106

Figure 4.29 Measurement of a) the area of the jet measured from $\xi_{*}=$ $0.1 \rightarrow \xi_{*}^{0}$ for PG (solid) and AG (dashed) and b) the final area of the jet after reconstruction. . . . . . . . . . . 108 
Figure 4.30 Turbulent eddy viscosity determined from the linear viscosity model for PG (a-c) and AG (d-f). The centerline (dotted) and JCB (dashed) are included for visual comparison. The eddy viscosity has been represented on a logarithmic scale to allow for a broader range of values. . . . . . . . . . . . . . . 110

Figure 4.31 The method of evaluating the turbulent eddy viscosity on the relative axis using the shear stress and mean strain rate profile across the width of the jet for one PG and one AG case. . . . 112

Figure 4.32 Comparison of the turbulent eddy viscosity from linearly fitting the profiles in Figure 4.31 to the mean shear, two- and three-component linear eddy viscosity model within the jet from Figure 4.30. . . . . . . . . . . . . . . . . . . . . . . 113

Figure 4.33 Eddy viscosity as a function of the scaled jet length $\xi / \xi^{0}$ for low inflow turbulence (top panel) and $\xi / \xi^{F F}$ for high inflow turbulence (bottom panel). . . . . . . . . . . . 114

Figure 4.34 Turbulent dissipation calculated directly as $\varepsilon=2 \nu\left\langle s_{i j} s_{i j}\right\rangle$ for three low (a-c) and three high (d-f) cross-flow turbulence intensities. . . . . . . . . . . . . . . . 117

Figure 4.35 Separated regions of the flow-field and streamlines for evaluation of $D_{L L}$. The inflow (I) was evaluated using streamwise component, $u$. The jet (II) was evaluated using the velocity component tangential to the centerline, $v_{r}=u \sin \left(\theta_{C L}\right)+$ $v \cos \left(\theta_{C L}\right)$. The wake region (III) was evaluated in the direction of the flow at the current location, $v_{r}=u \sin \left(\theta_{\phi}\right)+v \cos \left(\theta_{\phi}\right) .118$ 
Figure 4.36 Corrected dissipation within each region determined through second order structure functions, $D_{L L}(\mathrm{x}+r)$. The inflow $(\mathrm{a}, \mathrm{d})$ was evaluated downstream $r\left(x_{*}\right)$, the jet (b, e) was evaluated tangential to the jet centerline $r\left(\xi_{*}\right)$, and the wake region $(\mathrm{c}, \mathrm{f})$ was evaluated using streamlines $r\left(\phi_{*}\right) . \quad \ldots \ldots \ldots 120$

Figure 4.37 Gradient dissipation correction factor, $\beta=\varepsilon_{s, c} / \varepsilon_{g, m}$, for several cases. . . . . . . . . . . . . . . . . . . . 121

Figure 4.38 The average ratio of turbulent production $\mathcal{P}$ to turbulent dissipation, $\varepsilon_{s, c}$ within three regions. Region I is averaged in the streamwise direction and plotted as a function of height, $y_{*}$ (a, d). Region II is averaged across the width of the jet and plotted as a function of jet length, $\xi_{*}(\mathrm{~b}, \mathrm{e})$. Region III is averaged vertically and plotted as a function of downstream location, $x_{*} \ldots \ldots \ldots \ldots \ldots \ldots \ldots \ldots \ldots$

Figure 4.39 The Kolmogorov length scale in diameters, $\eta_{*}$, determined by the MSFM. The scale was averaged across the width of the jet and plotted as a function of scaled jet length $\xi / \xi^{0} \ldots \ldots$. . . 123

Figure 4.40 Closure argument $C_{\mu}$ determined from the definition $\nu_{T} \equiv$ $C_{\mu} k^{2} / \varepsilon \ldots \ldots \ldots \ldots \ldots \ldots \ldots \ldots$

Figure 4.41 Closure argument $C_{\mu}$ determined from the definition $\nu_{T} \equiv$ $C_{\mu} k^{2} / \varepsilon$ in inflow, averaged as a function of elevation $y_{*}(\mathrm{a}, \mathrm{b})$, and the wake, averaged downstream to evaluate as a function of $y / y^{0}(\mathrm{c}, \mathrm{d})$. 
Figure 4.42 Critical points identified on the $x-z$ plane. Classified by the surrounding flow, the presence of nodes $(\mathrm{a}, \mathrm{e})$, saddles $(\mathrm{b}, \mathrm{f})$ and foci (c, g) are concentrated along the leading edge shear layer and lee-side wake region. Additional statistics on flow orientation are included $(\mathrm{d}, \mathrm{h}) \ldots . . . . . . . . . . . .131$

Figure 4.43 Methods of identification of leading edge shear layer critical points. At each segment of jet length, critical points were sorted and the distance off the centerline, $r_{c p}$ was determined (a, d). The distributions of $r_{c p}$ were also calculated (b, c). The mean critical point distance, $\bar{r}_{c p}$, was plotted (a, d), aligning with the leading edge JCB. . . . . . . . . . . . . . . 132

Figure 4.44 Critical points classified as "unstable foci" within the nearfield wake of the jet for three PG and three AG cases. . . . . . 133

Figure 4.45 Critical point locations for $R_{v}=14$ at each elevation. The jet JCB (white) was included for spatial reference. . . . . . . . 134

Figure 4.46 The instantaneous velocity field (left) and $Q$-criterion (right) within the wake of the jet. Critical points (red) were classified by the surrounding flow. For the pictured set of critical points, a repelling saddle (blue) and repelling node (green) surround an entraining node. . . . . . . . . . . . . . 135 
Figure 4.47 Two-point correlation fields evaluating equation (2.75) along the leading edge JCB (dashed) for a) $\phi_{i}=\psi_{j}=u_{r}^{\prime}$ and c) $\phi_{i}=$ $\psi_{j}=v_{r}^{\prime}$. Auto-correlation functions were interpolated along the dashed red lines. The resulting auto-correlation curves $R_{u u}(r)$ (b) and $R_{v v}(r)(\mathrm{d})$ were integrated to yield the length scale of the coherent structures. . . . . . . . . . . . 137

Figure 4.48 The length scale of coherent structures evaluated at the leading edge JCB with the normal displacement vector directed outward. The radial autocorrelation function, $R_{u u}(r)$ (from Figure 4.47b), was integrated from the peak to the first zero crossing. . . . . . . . . . . . . . . . . . 139

Figure 4.49 The integral length scale evaluated at the JCB with the displacement vector directed upstream and into the jet. The radial autocorrelation function, $R_{u u}(r)$ (from Figure 4.47), was integrated from the peak to the first zero crossing. . . . . . . . 139

Figure 4.50 Locations of similar features across cases located within the jet $(\circ)$, the near-wake recirculation region $(\diamond)$, and the down-

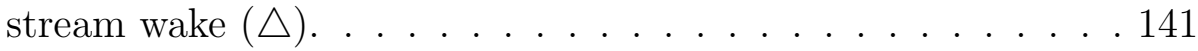

Figure 4.51 Auto-correlation of coherent structures, $R_{u u, r}$, for the streamwise fluctuating field within the wake at $x_{*}=10$ for three PG and three AG cases. . . . . . . . . . . . . . . . . 142

Figure 4.52 Auto-correlation of coherent structures, $R_{u u, r}$, for the streamwise fluctuating field within the near-wake recirculation region for three PG and three AG cases. . . . . . . . . . . . . . 143 
Figure 4.53 Two-point correlation, $R_{u u, r}$, evaluated at the leading edge $\operatorname{JCB}(\mathrm{a}, \mathrm{d})$, centerline $(\mathrm{b}, \mathrm{e})$ and lee-side $\operatorname{JCB}(\mathrm{c}, \mathrm{f})$ at $\xi^{0}$ for one PG and one AG case. . . . . . . . . . . . . . . . . . . . . 144

Figure 4.54 Plume centerline and shear layer output from Plumeria [8] identifying the dependence on $\alpha$ and $\beta$ for $R_{v}=14$. . . . . 148

Figure 4.55 Evaluation of the fit quality through residual analysis. For a constant $\alpha$, a range of $\beta$ were compared to experimental centerline data. The best $\beta$ for each $\alpha$ was determined (inset plot)......................... . . 149

Figure 4.56 Optimal $\alpha$ and $\beta$ to predict centerline (solid), center-plane area (dashed) and near-field volume (dotted) for three inflow velocities. Computed $\alpha$ and $\beta$ from mean flow statistics (red) are included for comparison. . . . . . . . . . . . . . . . 150

Figure 4.57 Entrainment coefficients a) $\alpha$ and b) $\beta$ for low and high inflow turbulence. The jet was separated and the near- and far-field

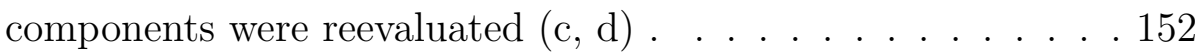

Figure 4.58 Entrainment coefficients in polar coordinates from $y_{*}=1 \rightarrow 6$. The leading edge of the jet is at $\theta=180$ for simplified recurring calculations. . . . . . . . . . . . . . . . . . . 154

Figure 4.59 Entrainment coefficients extracted from the mean flow statistics a) as a function of elevation, and b) as a single solution for each case. . . . . . . . . . . . . . . . . . 155 
Figure 4.60 Comparison between the Plumeria outputs and experimental data for the cross-sectional area $(\mathrm{a}, \mathrm{b})$ and the final rise difference, $\Delta y,(\mathrm{c}, \mathrm{d})$ at the end of the experiment centerline. . . 156

Figure 6.1 Simplified model complications for bending plume from Mastin [9, fig. 1]. . . . . . . . . . . . . . . . . . . 165

Figure 6.2 Cartoon of entrainment processes at the edge of the shear layer of a jet from Philip \& Marusic [10, fig. 6]. . . . . . . . . . . 168 


\section{Chapter 1}

\section{Introduction}

Advances in the study of turbulence have improved the ability for engineers and scientists to predict complex fluid interactions and mixing through simplified models. When the most subtle features of complex flows are accurately represented, it is possible to adapt the findings directly to engineering applications or to safeguard the public from natural disasters. One such complex fluid interaction is jets in cross-flow (JICF), a phenomenon that occurs when a fluid injected into oncoming cross-flow undergoes subsequent mixing as the jet is carried downstream. JICF has been applied to controlled systems such as coolant dispersal in heated fluid [11] and on wind turbine blades [12]. Research breakthroughs in the study of JICF have yielded efficiencies for uniform mixing during fuel injection, as well as quantifying the amount of coolant required to guarantee safe operation temperatures. However, when scaling JICF to larger, less controlled environmental flows, practical applications must account for complex conditions and factors. This would include accurately predict-

ing everything from the spread of pollution from factory chimneys $[13,14,15]$ and wastewater drains [16], to expeditiously modelling the path and concentrations of volcanic plumes [17] over populated areas.

For these applications, a critical understanding of the rate that air is absorbed 


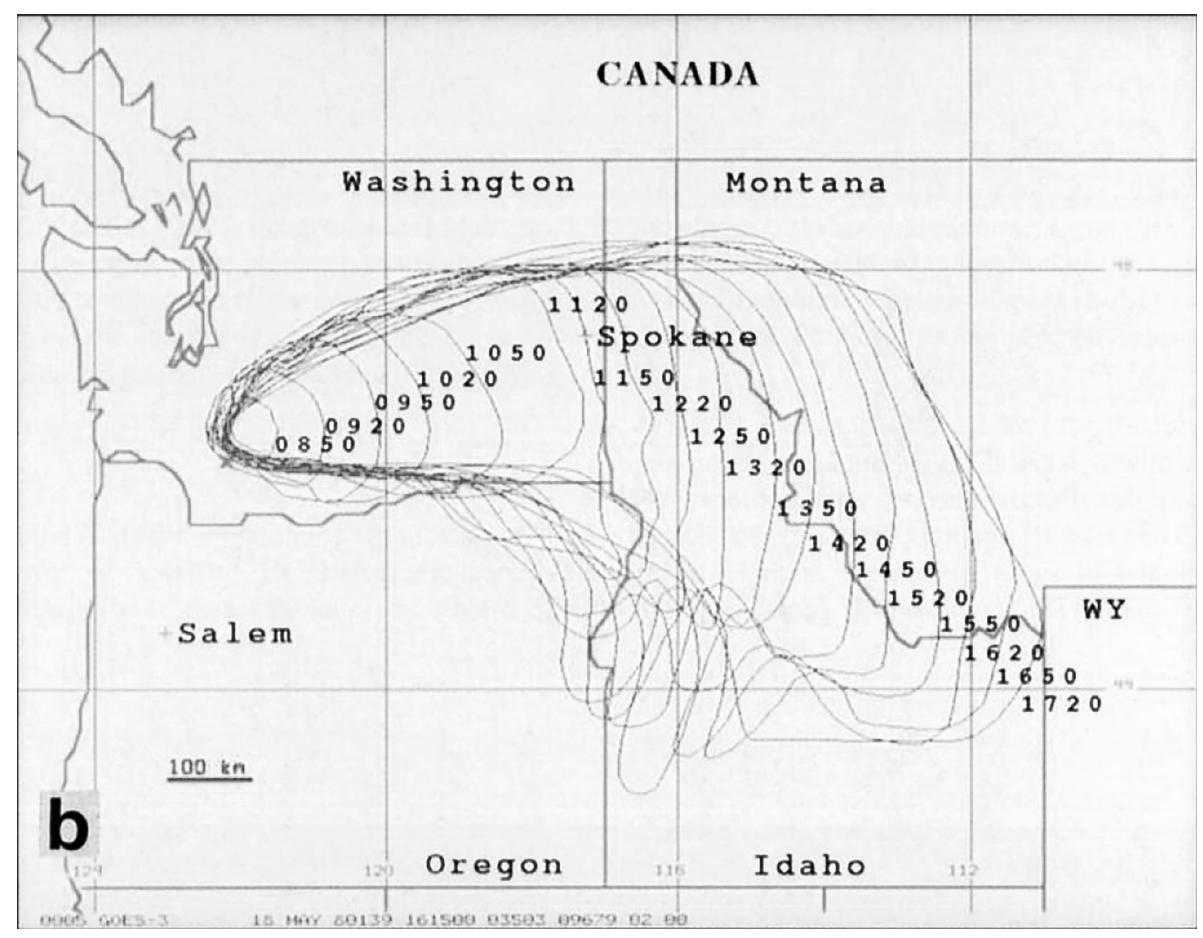

Figure 1.1: Volcanic plume spread after the 1980 Mt. St. Helens eruption from Holasek \& Self [1, p. 8474].

by the jet, a phenomenon known as entrainment, allows for prediction of the farfield concentration and extent. During a volcanic eruption, toxic plumes travel hundreds of miles over densely populated areas in a matter of hours (Figure 1.1). Therefore, rapid response time is the single most critical component when predicting the path and evolution of a plume. While understanding the complex interaction of plumes with the wind is essential, simplified models have allowed researchers to quickly describe the changes in plume concentration as ash approaches populated regions. Through decades of scientific study [18], it has been determined that when turbulent features in the flow are removed, it is possible to identify the underlying physics dictating the growth and expansion of flow within the shear layer.

Early work on descriptions of JICF focused on physical characteristics such as 
velocity and momentum ratios, as well as temperature and pressure profiles (see $[13,19,20,21,22,23,24])$. Empirical models were then developed to describe the trajectories [25] and velocity field [26]. The trajectories are characterized as a power law fit of the form $y=A x^{b}+y_{0}$, the coefficient, $A$, and exponent, $b$, scaled differently with downstream location. Near the jet, the trajectory curves have collapsed under the scaling parameter $R_{v}^{2} D$ as the jet still maintained high momentum to rise, despite the body forces imparted on it. Further downstream, a second scaling parameter, $R_{v} D$, was needed once the momentum of the jet had dissipated and was neutrally buoyant at the final rise height [19]. There have been attempts to relate the near and far-field, but ultimately they have been found to scale differently, with the near-field initially scaling as a turbulent jet and the far field scaling as a free-shear flow (see $[27,28,29,30]$ for some scaling relations). The transition between these regions occurs at approximately $\xi / R_{v}^{2} D=0.3[31]$, thereby indicating that a separation of the flow into two regions demonstrates subtle features that contribute to the near-field behavior are also essential in far-field, fully developed flows.

\subsection{Turbulent Entrainment}

While turbulent motions are chaotic by nature, methods to categorize these motions by noting similar behavioral characteristics has been theorized for decades [32]. Turbulence was assumed to be a stochastic process until Townsend theorized the presence of large eddies in energy equilibrium with a sub-structure that contains turbulent energy [33]. This led to the development of the modern theory which took a Lagrangian approach to view these "coherent structures" as regions of concentrated vorticity centered on an unstable line that move and deform as they travel in a fluid. 
These structures are present across a range of scales. Kolmogorov [34] theorized that the largest scales entrain energy, which is then transferred to smaller eddies before dissipating at the smallest scale, $\eta$. Because of this, it would be possible to measure entrainment by focusing solely on the largest coherent structures within the flow.

For JICF, turbulent features are present across a large range of scales. With highly turbulent flow contained within the shear layer with a fixed momentum profile and ambient or irrotational flow present outside, the growth of the jet must come from an outside source: ambient fluid that is drawn towards the jet and entrained at the shear layer through some method of transport [35]. Therefore, an accurate representation of entrainment requires an understanding of the flow patterns near the edges of the shear layer. As the ambient fluid approaches the edge of the jet, it comes into contact with turbulent jet at a thin boundary that separates fluids in different turbulent states known as the "laminar superlayer" [36]. This boundary is at the outermost edge of the region referred to as the turbulent/non-turbulent ( T/NT) interface. Initial investigations determined that the thickness of the $\mathrm{T} / \mathrm{NT}$ interface was on the order of the Taylor microscale [37], highlighting the importance of turbulence in the growth of the jet. A comparison of the local and global interface velocity yielded conflicting results, with the local interface velocity and thickness on the order of the Kolmogorov scale.

Investigations are further complicated by the fact that at any instantaneous moment, the T/NT interface has been found to be continuously deformed by large eddies within the turbulent region [2] (see sketch in Figure 1.2). This observation has led to the belief that the large vortices present in the turbulent flow engulfed irrotational fluid within this region, thereby expanding the shear layer [38, 39, 40]. 


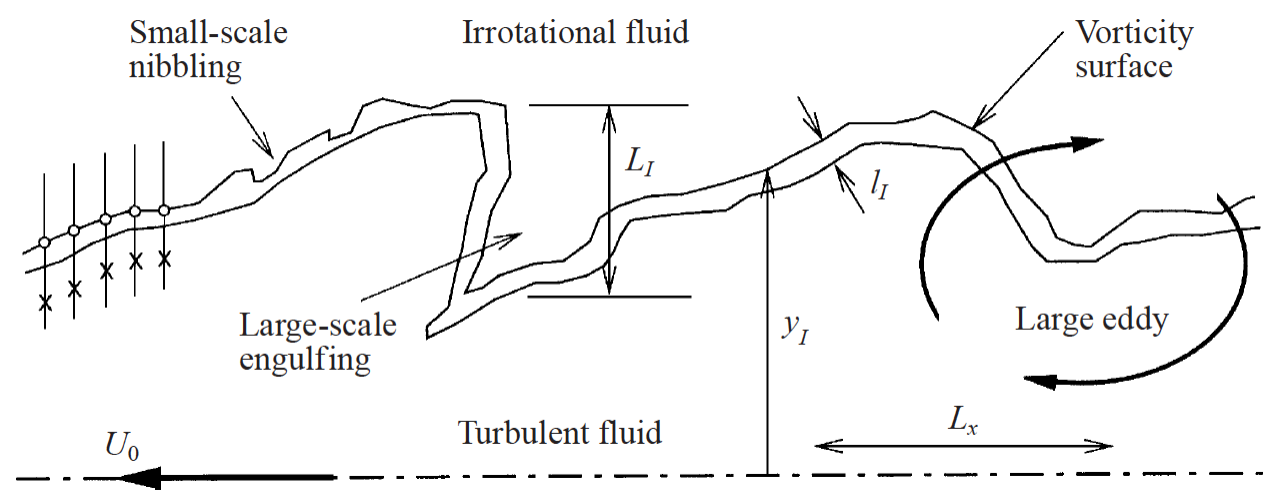

Figure 1.2: Sketch of the Turbulent/Non-turbulent interface and entrainment processes from Bisset et al. [2].

High resolution measurements have demonstrated that engulfment is not the primary source of entrainment, but rather, viscosity and molecular diffusion are the primary contributors at much smaller scales, known as "viscous nibbling" [41, 42]. For flows with large coherent structures, both processes are found to contribute to entrainment [10], with irrotational fluid drawn in through engulfment and nibbled by small scale eddies. Although the instantaneous movement of the T/NT interface was found to be independent of viscosity due to the deformation by large eddies $[43,44]$, the viscous term is the dominant contributor to turbulent entrainment, influencing the entrainment velocity [45] and the turbulent entrainment outside of the $\mathrm{T} / \mathrm{NT}$ interface [46]. In addition, it has been discovered that entrainment for a jet in potential flow is scale-independent as the entrainment velocity scales with the interface length [47].

\subsubsection{Vortex Systems}

While the smallest contributions to entrainment are significant in predicting turbulence behavior, the role of large scale coherent structures is essential to any descrip- 
tion of JICF. For an axisymmetric JICF, there is an immediate loss of self-similarity at the exit due to varying pressure around the jet profile [48]. Because the jet is a fluid rather than rigid body, these variations in pressure allow the jet to deform into four distinct complex vortex systems (see Figure 1.3).

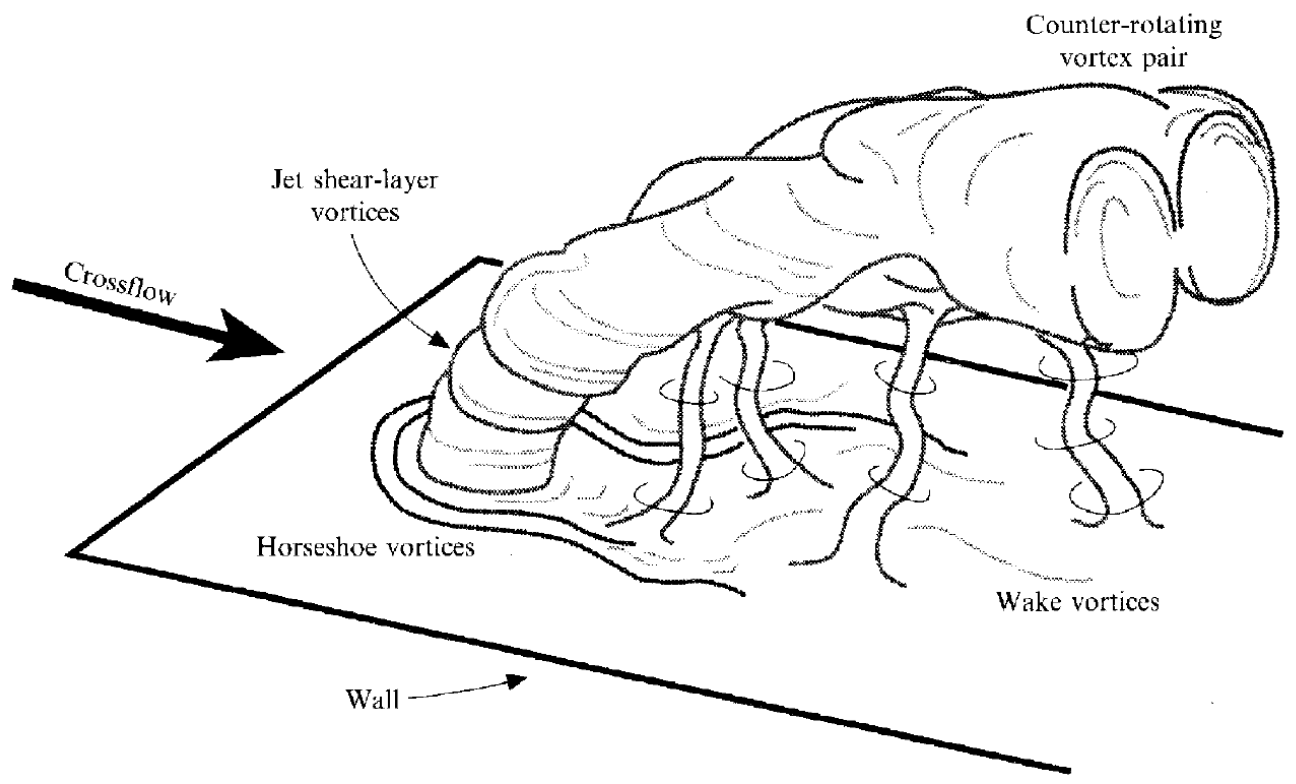

Figure 1.3: Cartoon schematic of the four dominant flow features for a jet in cross-flow from Fric \& Roshko [3, p. 2].

At the exit of the jet, vorticity is formed similar to a vortex ring [49]. These are the result of the Kelvin-Helmholtz instability of the shear layer [50, 51]. Due to the cross-flow pushing the jet downstream, these vortices are present only along the leading edge, and grow as the shear layer develops. These "shear layer vortices" are responsible for the deformation of the $\mathrm{T} / \mathrm{NT}$ interface, and similar to the axisymmetric roll-up present in vortex rings, they begin to form around the jet. Off the centerline, the cross-flow imparts the shear layer vortices with streamwise momentum, and as the vortices wrap around the jet, they converge on the lee-side and 
form two smaller vortices. Over the length of the jet, these vortices grow into two large counter-rotating vortices that are indistinguishable from the jet in the far-field. Several works have investigated and quantified the formation of these vortices (see Karagozian [52] and Cortelezzi \& Karagozian [53] for some examples), and they have been found to be dependent on the exit profile of the jet itself. Even with increases in shear layer thickness when the jet is allowed to fully develop, [54, 55], self similarity is achieved over the same downstream distance [56] for a circular exit. For more complex exit geometries, such as the fractal patterns studied in Breda \& Buxton [57], a suppression in large scale coherent structures and delay in development is observed in the near-field.

Moving attention upstream of the jet near the floor, a stagnation point is formed due to the high vertical momentum of the jet that prevents movement of cross-flow through the jet. It is here that a new vortex develops, centered on a critical point that entrains cross-flow. As this coherent structure is imparted with momentum from the cross-flow, its edges wrap around the jet to form a horseshoe vortex that extends downstream $[58,59]$. On the lee-side of the jet, the cross-flow moving around the jet (and drawn into the wake by the counter-rotating vortices) is imparted with vorticity and then shed downstream, forming a large "tornado like vortex" that extends from the wake boundary layer to the lee-side of the jet where it is entrained [3]. This structure is carried further downstream where it stretches and continues to entrain fluid within the boundary layer. Commonly compared to bluff body wakes, the shedding of vortices has been intensely investigated for decades [60]. While early research centered on circular profiles, it was the introduction of varying body shapes that allowed for the realization that sharp edges change the behavior 
of wake formation. A study by Higuchi et al. [61] of symmetric polygons observed vortex deformations when sharp edges were prominent. This was further confirmed in the work $\mathrm{Hu} \&$ Zhou [62] through PIV measurements of square profiles with various chamfered edges. And the works of Nedić et al. introduced complex shapes and fractal patterns that reduced the vortex shedding energy while increasing the duration of energy decay $[63,64,65]$.

The complexity of this work lies in an understanding that, at any instantaneous moment, the geometric profile of the jet will vary and the wake behavior will change. Within the wake, a critical point identified as the unstable focus redirects flow downstream $[59,66,67]$. The mean statistics of this location is fixed. But, within a single snapshot, this location may shift due to the variations in wake behavior. Present across all complex turbulent flows, critical points (or singularity) provide spatial locations where the magnitude of the velocity field is zero. Additionally, within a cross-section slice of a vortex, there will be a rotation of fluid focused around a critical point.

\subsection{Turbulence Modelling}

To gain a deepened understanding of how JICF can be accurately predicted using real world tools, it is necessary to understand the history behind modelling turbulent flows. After Taylor [68] applied correlation functions to rotating flows to satisfy the Navier-Stokes equations, there have been many other attempts to predict turbulent motions. Two different perspectives were successful, the most promising being the approximation of the smallest features through an eddy-viscosity term [69]. By creating a grid and defining all initial conditions (see $[70,71,72]$ for some examples), 
the Navier-Stokes equations could be solved directly to resolve all scales. This method, direct numerical simulations (DNS), required the grid to be on the order of the Kolmogorov scale. Because this was not possible for many years due to restrictions in computational power, a different approach was designed to instead define a grid greater than the smallest scales and resolve only the largest eddies. The turbulent features were then represented on a subgrid scale (SGS) and the Reynolds stress tensor could be resolved by filtering the Navier-Stokes equation [73, 74, 75]. This approach is known as large eddy simulation (LES).

However, because the smallest features are unresolved, they are approximated through a single term known as the turbulent eddy viscosity, $\nu_{T}$. Boussinesq [76] hypothesized that the "turbulent fluctuations are dissipative on the mean flow," indicating the Reynolds stress tensor is proportional to the mean strain rate, a theory that was later confirmed [77]. Other methods were developed to determine $\nu_{T}$ by relating different components within the system. For example, the $k-\epsilon$ model balanced the turbulent kinetic energy and the turbulent dissipation [78], while the Smagorinsky model used the grid size and mean strain rate with a different dimensionless coefficient [79].

The evolving theoretical frameworks then required rigorous testing to determine their validity. For example, the mean flow results of two JICF velocity ratios collected by Crabb et al. [80] and Andreopoulos \& Rodi [81] were used to validate simulations by Wille [82] and Wegner et al. [83] respectively. With more in-depth analysis of turbulent statistics within the jet, and with well established closure arguments, other simulations (see $[84,85,86]$ for some examples) confirmed the ability of LES to predict mixing and entrainment. 


\subsubsection{Low Order Modelling}

It is important to note that these modelling approaches are useful for smaller scale flows with simple conditions. For much larger domains, such as atmospheric mixing, many factors including terrain, wind variability and spatial resolution, make LES a less efficient predictive method. Instead, two different approaches have been employed to predict entrainment using simplified aerodynamic principles and an integral approach (see Figure 1.4). The first approach, which is primarily used for aerodynamic applications, models the system as a pair of counter-rotating vortices (see $[52,87]$ ), thereby providing finer accuracy by accounting for turbulent features. One drawback to this approach is higher computational cost. The second application, similar to the approach described above, involves modelling the jet as an entraining surface, where the rate of entrainment is summarized through transport analysis [4]. By removing the turbulent features from the flow, all subtle contributions are subsequently ignored.

Due to the complexities of entrainment across scales and interconnected vortex systems, it is essential to identify all velocity components and initial conditions. This requirement has made real time prediction difficult, especially under the conditions where any components are unresolved. To avoid this uncertainty, models have been simplified even further to approximate these velocity components, and the resulting mixing, as coefficients (see $[8,88,89,90]$ for some examples). Under

the revised theoretical framework based on the "Taylor entrainment assumption," for an axisymmetric turbulent jet exiting into ambient fluid, the expansion of the jet width is now determined through simplified momentum balances, as presented by Morton et a. [91]. Briggs [92, p. 335] noted: 

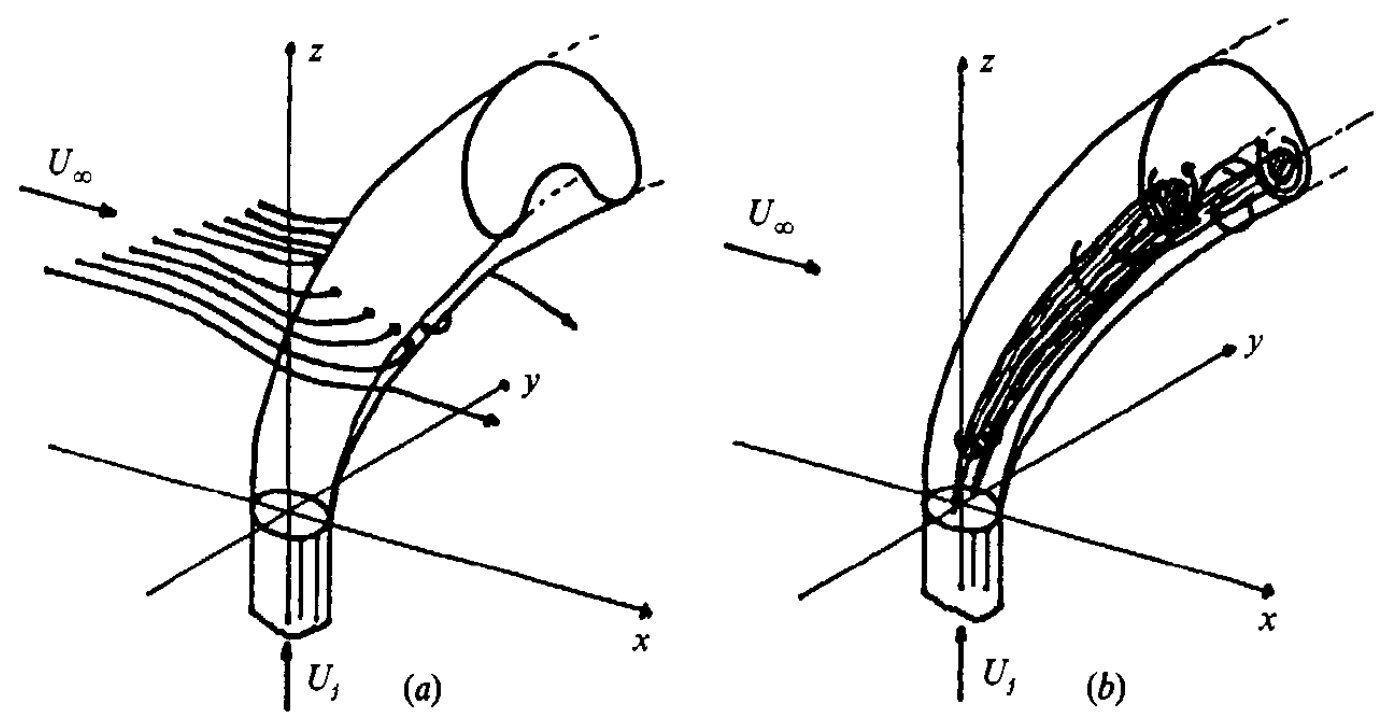

Figure 1.4: Approaches to simplified models for a jet in cross-flow from Coelho \& Hunt [4, p. 96]. Modelling the jet as a) an entraining surface, and b) as a pair of vortices removed complexity by summarizing turbulent contributions through transport and aerodynamic principles.

The Taylor assumption is based on the idea that, because turbulence in the plume is generated by the velocity shear between the plume and the ambient fluid, all turbulent velocities, including the entrainment velocity, scale locally to the mean plume vertical velocity.

This idea is further summarized through the proportionality coefficient, $\alpha$, defined as the ratio of entrained air (entering the plume) and centerline velocity. Testing under a variety of conditions has identified the entrainment velocity to be $\sim 5-6 \%$ of the centerline velocity, with variations for extreme cases [93, 94, 95]. While it is generally accepted for volcanic plumes as $\alpha=0.09$, Hewett et al. [96] found values up to $\alpha=0.15$ for special conditions.

Research has shown that in the presence of cross-flow, radial entrainment is one 
of several components that contribute to the net transport. This is especially true downstream in the far-field, where the jet is passively carried by the cross-flow and the centerline velocity is equivalent to the cross-flow. The cross-flow entrainment coefficient, $\beta$, which is defined as the ratio of the velocity normal to the centerline and the entrainment velocity [97], has now been added. And while the value of this coefficient is not as well constrained, it is generally accepted that $\beta=0.5$, but can vary between 0.4 and $1.0[20,96,98,99,100]$. Incorporation of both terms required the assumption they were additive [98].

Of course, calculations using inputs that exist over a range of values will lead to a significant difference in predictions of the far-field concentrations and shape [101, 102]. For many applications, this large range can result in differences over an order of magnitude, especially when initial conditions are not well constrained (Figure 1.5). Therefore, and investigation of the complex features through a simplified model would reduce these uncertainties by quantifying unresolved components.

\subsection{Creation of Experimental Turbulence}

Computational fluid dynamics has laid important groundwork for an understanding of the behavior of turbulence. It not only allows for a more refined visualization of complex interactions such as JICF, but it also helps better understand the underlying contributions to developing turbulence. With simplified models, the assumption of negligible free-stream turbulence ignores features that previous studies have shown contribute to entrainment and are the primary source of expansion of the shear layer. Additionally, research has shown most atmospheric conditions are turbulent with variability in both speed and direction. This poses a new question: 


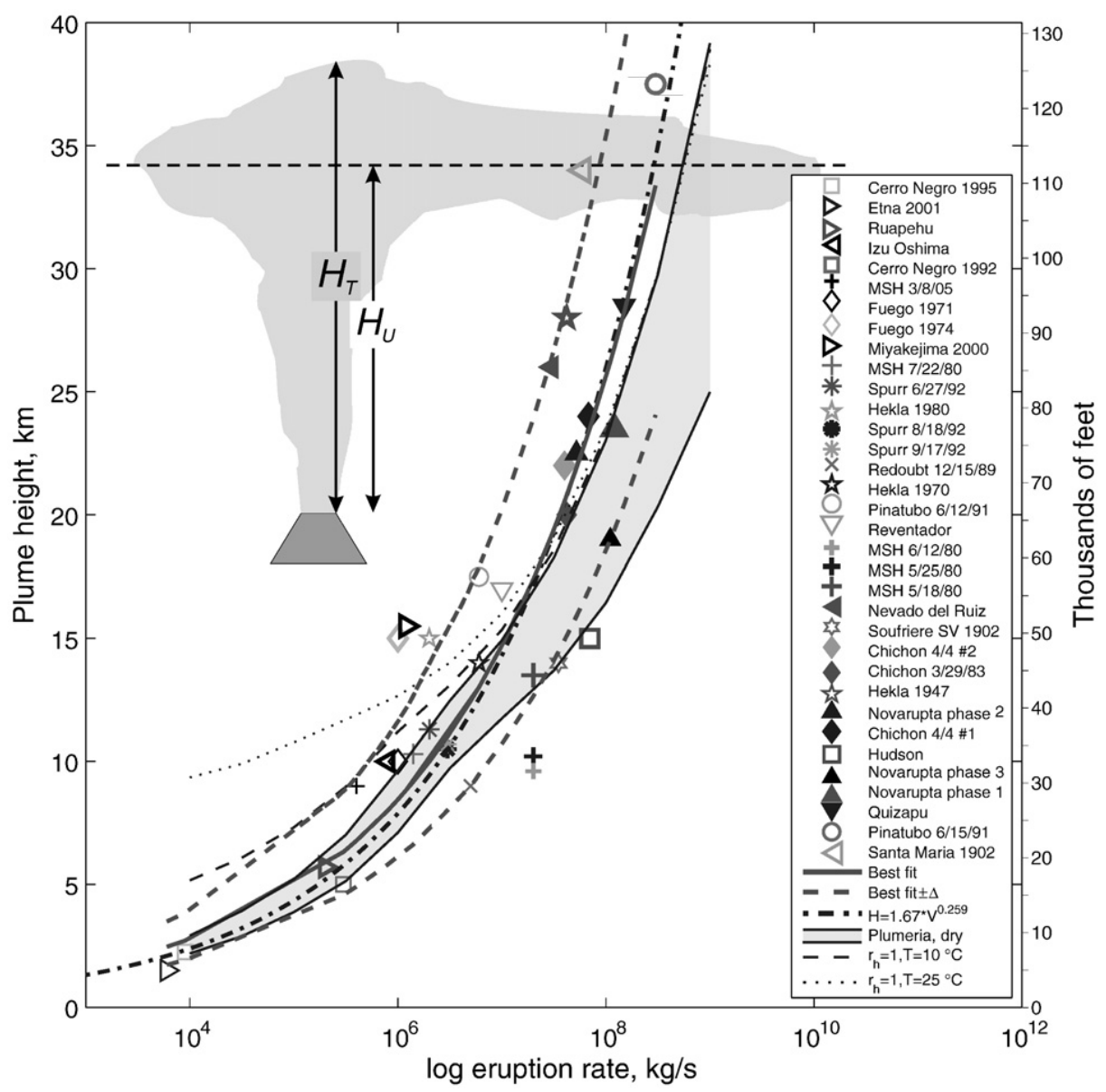

Figure 1.5: Volcanic plume data used for validation of volcanic plume models from Mastin et al. [5]. Using physical features of an eruption as inputs allowed comparison to converge on optimal configurations. Due to significant uncertainty, incorrect computations could have resulted in an order of magnitude error.

what effect does inflow turbulence have on the development of coherent structures and what are the consequences of inflow turbulence on entrainment?

For this complex system to be experimentally examined, a tightly controlled turbulent environment must be created within a test section. Historically, attaining uniform and fully developed turbulence for an experiment can be traced to the first 
passive element system devised by Simmons \& Salter [103]. It is known that turbulence is organized across multiple scales and many theories assume the turbulence is self-similar across them (see $[104,105,106,107])$. The authors placed horizontal and vertical strips in front of a test section to measure the turbulence. This passive grid system created small wakes behind the rods at the entrance to the test section, that converge into a fully turbulent inflow. With the goal of achieving higher Reynolds numbers, different approaches were employed, including adding a series of grids or differently shaped blockages. The creation of a fractal grid allowed for excitation at all scales [108, 109].

Even with these blockages, sufficiently large turbulence Reynolds numbers were not achieved, so a more direct agitation of the flow was attempted. In place of rods or patterns, blockages with controlled dynamic elements were used to excite the flow. Initially, two systems were developed: a series of controlled jets $[110,111]$ and vibrating grids $[112,113]$, but neither was able to provide uniform 3D turbulence in the test section. Then, in 1991, Makita \& Sassa [114] released the first results of an active grid system which utilized rows and columns of rotating winglets. By operating each rod independently, it became possible to simulate and control a variety of turbulent conditions in a wind tunnel including the atmospheric boundary layer [115]. This system was a breakthrough in the study of turbulent flows as it created homogeneous and quasi-isotropic turbulence with a large range of intensity levels.

In this dissertation, fundamental research for JICF was expanded upon through evaluation of the near-field in varying turbulent conditions. In forthcoming sections, the experimental setup and implementation are detailed, and subsequent analyses 
described. In the next chapter, differences in flow behavior through transport and characterization of physical features are examined. This is followed by an investigation across scales: first, identifying the energy content within the jet and extracting reduced order modeling terms to clarify unresolved contributions; and second, identifying the largest features in the flow using both instantaneous snapshots and auto-correlations. These observations are then used to support the uncertainty associated with the reduced order model, Plumeria. Together, these insights provide a comprehensive overview of near-field turbulence and serve as a foundation for future experimentation to better inform both engineering design and geophysical models. 


\section{Chapter 2}

\section{Theory and Methodology}

All methodology used for the quantification of turbulent contributions in JICF is herein outlined. It will begin with fundamental turbulent theory and an examination of fluid transport and entrainment, first approaching the problem through the application of the Navier-Stokes equations to describe transport and entrainment (Section 2.1). Next, through an investigation of well-established models, Section 2.2 will focus on reduced order models that account for turbulence contributions through closure terms (Section 2.3). After illustrating how turbulence is approx-

imated, an in depth examination of turbulent coherent structures on a variety of scales will be performed. This will include quantification of the turbulent dissipation (Section 2.4), identification of vortices through critical point analysis (Section 2.5), descriptions of the coherent structures through proper orthogonal decomposition (Section 2.6) and autocorrelation functions (Section 2.7). When viewed in total, these methodologies will summarize the most significant features in the turbulent interaction.

The fundamental theories of turbulence are continuously evolving. This is primarily due to the complexity of the natural state of turbulence, described as the "state of fluid motion which is characterized by apparently random and chaotic 
three-dimensional vorticity" [35, p. 11]. When a flow is characterized as turbulent, it is difficult to predict the motion at any instantaneous moment. Instead, a turbulent signal is broken into components through Reynolds decompositon, defined as

$$
\tilde{u}(t)=\bar{U}+u^{\prime}(t)
$$

For a velocity signal $\tilde{u}(t)$, the average and fluctuating components, $\bar{U}$ and $u^{\prime}$, are separated.

By describing the flow as an average component, highly turbulent flows are more easily predicted by eliminating the inherent randomness. From the fluctuating component, the turbulence can then be characterized through the Reynolds stresses. While the average of the fluctuations are negligible $\left(\overline{u^{\prime}}=0\right)$, the average of two

fluctuating components yields the Reynolds stress tensor, $\overline{u_{i}^{\prime} u_{j}^{\prime}}$. This is utilized to describe either the directional magnitude of the fluctuations through the normal stress $(i=j)$ or the magnitude of orthogonal components through the shear stresses $(i \neq j)$.

\subsection{Transport}

When the role of turbulence is important for analysis of transport, the Navier-Stokes momentum equation provides descriptions of different features in the flow.

$$
\rho\left[\frac{\partial u_{i}}{\partial t}+u_{j} \frac{\partial u_{i}}{\partial x_{j}}\right]=-\frac{\partial p}{\partial x_{i}}+\frac{\partial \tau_{i j}}{\partial x_{j}} .
$$

With no direct solution to the Navier-Stokes equation, the decompositon into mean and fluctuating components allows analysis of the behavior of the turbulence. 
For 2.2, this produces the Reynolds-Averaged Navier-Stokes (RANS) momentum equation

$$
\underbrace{\bar{U}_{j} \frac{\partial \bar{U}_{i}}{\partial x_{j}}}_{A}=\underbrace{-\frac{1}{\rho} \frac{\partial \bar{p}}{\partial x_{i}}}_{B}+\underbrace{\frac{1}{\rho} \frac{\partial \tau_{i j}}{\partial x_{j}}}_{C}-\underbrace{\overline{u_{j}^{\prime} \frac{\partial u_{i}^{\prime}}{\partial x_{j}}}}_{D} .
$$

This equation consists of four terms, the inertial term known as convection (A), the pressure term (B), the viscous term $(\mathrm{C})$ and the Reynolds stress term known as diffusion (D). If all of the components are known, it is possible to create a momentum budget to compare the dominant process within the flow. When evaluating velocity fields alone, it is difficult to resolve all four components. Instead a comparison between terms provides a distinction on the greatest contributor to momentum.

The convection and diffusion terms (referred to as $M_{I}$ and $M_{R S}$ ) are chosen to allow for a comparison between the inertial and Reynolds stress contributions to momentum. They are defined as

$$
M_{I, i j}=\bar{U}_{j} \frac{\partial \bar{U}_{i}}{\partial x_{j}}
$$

and

$$
M_{R S, i j}=\overline{u_{j}^{\prime} \frac{\partial u_{i}^{\prime}}{\partial x_{j}}} .
$$

For a closed control volume in space, the transport can be evaluated and compared to determine the dominant features in the flow. The volume integral is transformed into a surface integral with the divergence theorem, which allows the convective term to be represented as

$$
M_{I}=\int_{S}\left(\bar{U}_{j} \bar{U}_{i}\right) \cdot n_{i} \mathrm{~d} S
$$


and the Reynolds stress term as,

$$
M_{R S}=\int_{S}\left(\overline{u_{i}^{\prime} u_{j}^{\prime}}\right) \cdot n_{i} \mathrm{~d} S
$$

where $n_{i}$ is the unit vector normal to the surface, $S$.

For the energy transport, the momentum terms are multiplied by the velocity component, $\bar{U}_{i}$, producing the inertial energy and energy flux terms,

$$
E_{I, i j}=\bar{U}_{j} \frac{\partial K}{\partial x_{j}},
$$

and

$$
E_{R S, i j}=\frac{\partial}{\partial x_{j}}\left(\overline{u_{i}^{\prime} u_{j}^{\prime}} \bar{U}_{i}\right)
$$

where $K$ is the mean kinetic energy (per unit mass), defined as $K=1 / 2\left(\bar{U}_{i} \bar{U}_{i}\right)$. Similar to the momentum terms, a volume integral is taken over the same closed control volume, allowing the terms to be written as

$$
E_{I}=\int_{S}\left(\bar{U}_{i} K\right) \cdot n_{i} \mathrm{~d} S
$$

and

$$
E_{R S}=\int_{S}\left(\overline{u_{i}^{\prime} u_{j}^{\prime}} \bar{U}_{i}\right) \cdot n_{i} \mathrm{~d} S
$$

Comparisons across cases require reorientation of components onto a uniform axis. Velocity components are transformed relative to the jet centerline and spatially defined as a function of the distance off the centerline, $r$, and the length of the jet, $\xi$, as defined in Appendix B. Once defined, the transport terms are evaluated along 


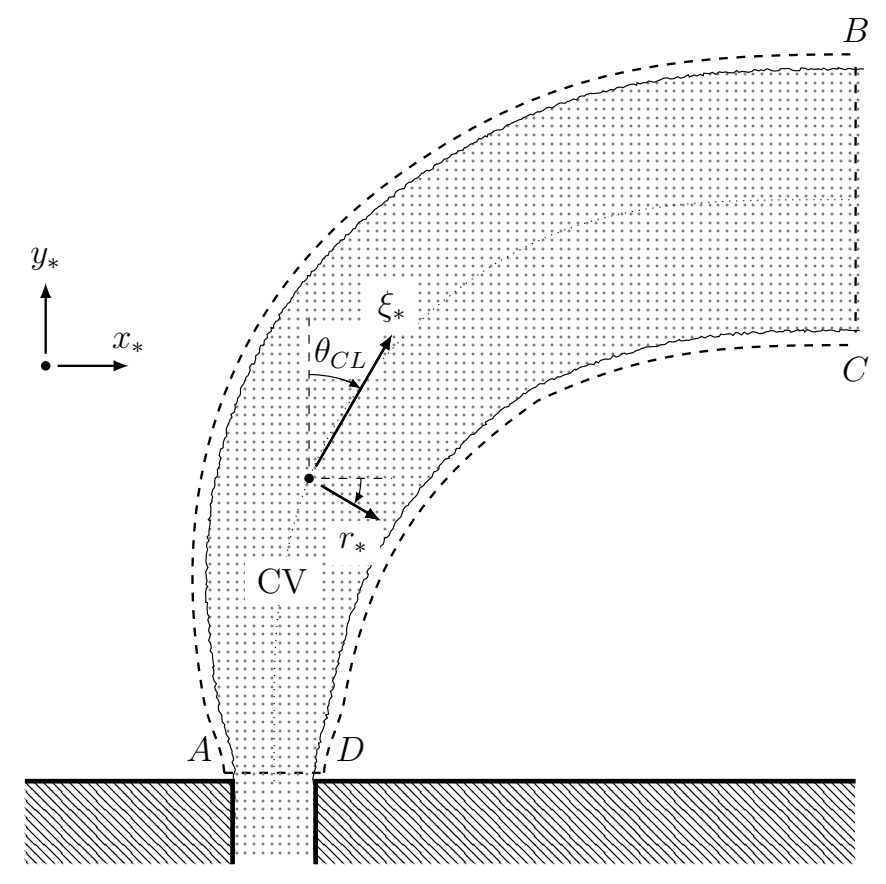

Bending Jet Axis

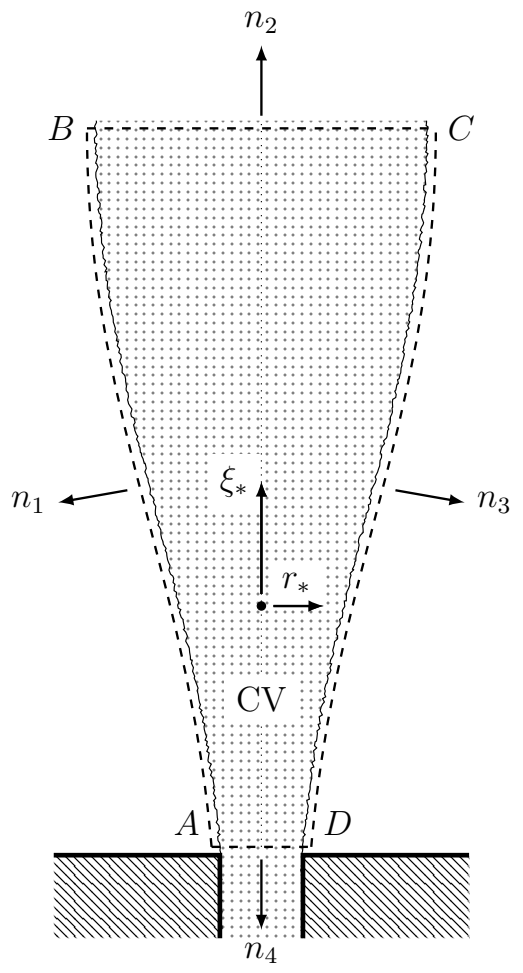

Relative Jet Axis

Figure 2.1: Transform of a bending jet from cartesian to relative coordinates.

a common feature across all cases, which for this system is the edge of the shear layer on the leading edge (LE) and lee-side (LS) represented by surface $\overrightarrow{A B}$ and $\overrightarrow{D C}$ on Figure 2.1.

The momentum and energy equation terms are then evaluated along this surface using the transformed velocity components. For the normal component, positive is defined into the control volume to easily differentiate entrained momentum and energy from losses, hence $n_{i, L E}=1$ and $n_{i, L S}=-1$. The full expansion of the momentum terms is represented as

$$
M_{I}=\int_{A}^{B}\left(\bar{U}_{r}^{2}+\bar{U}_{r} \bar{V}_{r}+\bar{U}_{r} \bar{W}_{r}\right) \mathrm{d} S-\int_{D}^{C}\left(\bar{U}_{r}^{2}+\bar{U}_{r} \bar{V}_{r}+\bar{U}_{r} \bar{W}_{r}\right) \mathrm{d} S
$$


and

$$
M_{R S}=\int_{A}^{B}\left(\overline{u^{\prime} u^{\prime}}+\overline{u^{\prime} v^{\prime}}+\overline{u^{\prime} w^{\prime}}\right) \mathrm{d} S-\int_{D}^{C}\left(\overline{u^{\prime} u^{\prime}}+\overline{u^{\prime} v^{\prime}}+\overline{u^{\prime} w^{\prime}}\right) \mathrm{d} S .
$$

Similarly, expansion of the energy terms produces,

$$
E_{I}=\int_{A}^{B} \bar{U}_{r} K \mathrm{~d} S-\int_{D}^{C} \bar{U}_{r} K \mathrm{~d} S
$$

and

$$
E_{R S}=\int_{A}^{B}\left({\overline{u^{\prime} u^{\prime} U_{r}}}+{\overline{u^{\prime} v^{\prime} V_{r}}}+{\overline{u^{\prime} w^{\prime} W_{r}}}\right) \mathrm{d} S-\int_{D}^{C}\left({\overline{u^{\prime} u^{\prime} U_{r}}}+{\overline{u^{\prime} v^{\prime} V_{r}}}+{\overline{u^{\prime} w^{\prime} W_{r}}}\right) \mathrm{d} S .
$$

Evaluated at each shear layer, the transport of momentum and energy through the jet distinguish the differences between inertial and Reynolds stress contributions for various inflow conditions.

\section{$2.2 \quad$ Reduced Order Entrainment Models}

While turbulent contributions are essential in descriptions of entrainment for JICF, reduced order models make significant assumptions to simplify the flow and remove the subtle features. For applications such as volcanic plumes (commonly referred to as maintained plumes), these models are ideal for real time predictions of trajectory and mixing rates. The theory behind the entrainment of air on maintained plumes in an ambient field begins with an evaluation of the change in mass flux as a function of the jet length, $\xi[91]$.

$$
\frac{d M}{d \xi}=2 \pi r \rho \alpha u_{c}
$$


where $\alpha$ is the radial entrainment coefficient, defined as $\alpha=u_{e} / u_{c}$ where $u_{e}$ is the entrainment velocity and $u_{c}$ is the centerline velocity. Evaluated at the shear layer edge, this coefficient has been studied thoroughly, with a commonly accepted value at $\alpha \approx 0.09$ for most conditions. When cross-flow is introduced and the plume bends downstream, two components of velocity are present at the shear layer. First proposed by Hoult et al., a component normal and tangential to the flow will each have its own entrainment coefficient, and they are additive [98]. This second component, $\beta$, was used to describe the rate of cross-flow entrainment into both a momentum-driven jet, as well as a buoyancy driven jet.

For a bending jet in a uniform horizontal cross-flow, the entrainment velocity can be defined as the inward velocity of ambient air that is entering the jet at the $\mathrm{T} / \mathrm{NT}$ interface. This is generally quantified as

$$
u_{e}=\alpha\left|u_{c}-u_{\infty} \cos \theta_{C L}\right|+\beta\left|u_{\infty} \sin \theta_{C L}\right|
$$

where $\theta_{C L}$ is the plume angle relative to the cross-flow. Models such as Plumeria [9] simplify the calculations by using this entrainment velocity to evaluate the mass flux along the length of the plume by incorporating both radial and cross-flow entrainment at the TNTI,

$$
\frac{d M}{d \xi}=2 \pi r \rho\left[\left(\alpha\left|u_{c}-v_{\|}\right|\right)^{n}+\left(\beta\left|v_{\perp}\right|\right)^{n}\right]^{1 / n}
$$

where (in our $2 \mathrm{D}$ case) $v_{\|}=u_{\infty} \cos \theta_{C L}$ and $v_{\perp}=u_{\infty} \sin \theta_{C L}$. The $n$ exponent is an empirical value that is commonly taken as $n=1.5$. For varying density gases within the plume (a common occurrence in volcanic eruptions), two forms of $\rho$ are 
used:

$$
\begin{array}{ll}
\rho=\rho_{\infty} & \text { when } \rho_{j}<\rho_{\infty} \text { (positively buoyant), } \\
\rho=\sqrt{\rho_{j} \rho_{\infty}} & \text { when } \rho_{j}>\rho_{\infty} \text { (negatively buoyant), }
\end{array}
$$

where $\rho_{j}$ and $\rho_{\infty}$ are the plume density and ambient air density respectively.

Without the known velocity components, the mass flow rate, $\mathfrak{M}$, is evaluated as a system of equations with the momentum and energy flux for buoyant plumes,

$$
\begin{gathered}
\frac{d \mathfrak{R}_{x}}{d \xi}=\frac{d M}{d \xi} U \\
\frac{d \mathfrak{M}_{y}}{d \xi}=\frac{d M}{d \xi} V \\
\frac{d \mathfrak{R}_{z}}{d \xi}=\pi r^{2}\left(\rho_{\infty}-\rho_{j}\right) g \\
\frac{d E}{d \xi}=\frac{d}{d \xi}\left[M\left(\frac{u^{2}}{2}+g Z+h_{j}\right)\right]=\left(g Z+h_{\infty}\right) \frac{d M}{d \xi}
\end{gathered}
$$

where $h$ is the specific enthalpy and $Z$ is the elevation. The entrainment coefficients provide closure for the system.

\subsection{Large Eddy Simulation}

When modelling turbulence, there are two common approaches. The first is directly

solving the Navier-Stokes equations from a known physical state referred to as Direct Numerical Simulation (DNS). The second approach simulates the largest scales and approximates the smallest scales to provide a time independent solution referred to as Large Eddy Simulation (LES). Both of these systems use the momentum and 
continuity equations defined as,

$$
\begin{aligned}
& \frac{\partial u_{i}}{\partial t}+\frac{\partial}{\partial x_{j}}\left(u_{i} u_{j}\right)=-\frac{\partial p}{\partial x_{i}}+\nu \frac{\partial}{\partial x_{j}}\left(\frac{\partial u_{i}}{\partial x_{j}}\right), \quad i, j=1,2,3 \\
& \frac{\partial u_{i}}{\partial x_{i}}=0
\end{aligned}
$$

to solve for the state of the flow. While DNS provides the most accurate results for turbulent flows, the fact that all scales must be included carries significant computational expense. Therefore, the ability to use LES accurately can be beneficial to any discipline more focused on the larger scale features and steady solution of the flow.

When modelling these flows, it is important to account for these scales. For LES, the simulation must be defined on a subgrid of length $\Delta x$, that is larger than the smallest physical scales known as the Kolmogorov microscale, $\eta$. For a flow that is statistically homogeneous and isotropic, the difference between $\eta$ and the most energetic scales, $L$, are on the order of

$$
\frac{L}{\eta}=O\left(\operatorname{Re}^{3 / 4}\right)
$$

When predicting the behavior of turbulence, it is vital to recognize the significance of both of these scales. In this situation, to resolve both scales for a Reynolds number of $\sim 16,000$ (the Reynolds number of the jet in this work) assuming $L=D$, $\sim 10^{9}$ grid points would be required per unit volume $\left(D^{3}\right)$. To work around this, the most common approach is to filter out the smallest scales and determine a discrete solution, $u_{d}$, whose difference from the exact solution, $u$, is minimized. This means 
that the general conservation equation,

$$
\frac{\partial u}{\partial t}+F(u, u)=0
$$

is approximated as

$$
\frac{\delta u_{d}}{\delta t}+F\left(u_{d}, u_{d}\right)=0
$$

To reduce the error, $e\left(u, u_{d}\right)$, it is decomposed into three components,

$$
e\left(u, u_{d}\right)=e_{\Pi}\left(u, u_{d}\right)+e_{d}\left(u, u_{d}\right)+e_{r}\left(u, u_{d}\right)
$$

where $e_{\Pi}, e_{d}$ and $e_{r}$ are the projection error, discretization error and resolution error, respectively. These all account for the approximations made on the spatial filtering [116]. Instead of completely ignoring the smallest scales, the errors are reduced by predicting the features in the flow that cannot be resolved.

For a function that is resolved in both space and time, $\phi(\boldsymbol{x}, t)$, a high-pass filter is defined as

$$
\bar{\phi}(\boldsymbol{x}, t)=\int_{-\infty}^{+\infty} \int_{-\infty}^{+\infty} \phi\left(\boldsymbol{\xi}, t^{\prime}\right) G\left(\boldsymbol{x}-\boldsymbol{\xi}, t-t^{\prime}\right) d t^{\prime} d^{3} \boldsymbol{\xi}
$$

where $G$ is the convolution kernel that is specific to the filter used and associated with the cutoff scales for time and space, $\bar{\Delta}$ and $\bar{\tau}_{c}[30]$. When (2.28) is applied to 
(2.22) and (2.23), the filtered equations yield

$$
\begin{aligned}
& \frac{\partial \bar{u}_{i}}{\partial t}+\frac{\partial}{\partial x_{j}}\left(\overline{u_{i} u_{j}}\right)=-\frac{\partial \bar{p}}{\partial x_{i}}+\nu \frac{\partial}{\partial x_{j}}\left(\frac{\partial \bar{u}_{i}}{\partial x_{j}}\right), \quad i, j=1,2,3 \\
& \frac{\partial \bar{u}_{i}}{\partial x_{i}}=0 .
\end{aligned}
$$

Because this equation includes the non-linear term $\overline{u_{i} u_{j}}$, an additional step was needed. Leonard[117] was able to further decompose the velocity in this term,

$$
\begin{aligned}
\overline{u_{i} u_{j}} & =\overline{\left(\bar{u}_{i}+u_{i}^{\prime}\right)\left(\bar{u}_{j}+u_{j}^{\prime}\right)} \\
& =\overline{\bar{u}_{i} \overline{u_{j}}}+\overline{\bar{u}_{i} u_{j}^{\prime}}+\overline{\bar{u}_{j} u_{i}^{\prime}}+\overline{u_{i}^{\prime} u_{j}^{\prime}} \\
& =\overline{\bar{u}_{i} \overline{u_{j}}}-\tau_{i j}+\frac{1}{3} \eta_{k k} \delta_{i j},
\end{aligned}
$$

where $\tau_{i j}$ is the subgrid residual stress tensor defined as $\tau_{i j}=-\left(\eta_{i j}-\frac{1}{3} \eta_{k k} \delta_{i j}\right)$ and $\eta_{i j}=\overline{\bar{u}_{i} u_{j}^{\prime}}+\overline{\bar{u}_{j} u_{i}^{\prime}}+\overline{u_{i}^{\prime} u_{j}^{\prime}}$. With all terms not exclusively dependent on large scales grouped into one term, the momentum equation is rewritten in the filtered form

$$
\frac{\partial \bar{u}_{i}}{\partial t}+\frac{\partial}{\partial x_{j}}\left(\bar{u}_{i} \bar{u}_{j}\right)=-\frac{\partial \bar{p}}{\partial x_{i}}+\nu \frac{\partial}{\partial x_{j}}\left(\frac{\partial \bar{u}_{i}}{\partial x_{j}}+\frac{\partial \bar{u}_{j}}{\partial x_{i}}\right)-\frac{\partial \tau_{i j}}{\partial x_{j}}
$$

For LES, $\tau_{i j}$ is used to summarize all of the smallest scale turbulent contributions that cannot be resolved. These contributions must be incorporated through "subgrid scale" turbulence models; these small scale features are summarized through a single term, $\nu_{T}$, known as the bulk eddy viscosity.

There are different levels of complexity to determine $\nu_{T}$. One of the most common approaches is the linear viscosity model, which relates the Reynolds stresses to the 
strain rate through the eddy viscosity, $\nu_{T}$,

$$
\tau_{i j}-\frac{1}{3} \tau_{k k} \delta_{i j}=-2 \nu_{T} S_{i j}
$$

where $S_{i j}$ is the strain rate tensor defined as $\left(\partial u_{i} / \partial x_{j}+\partial u_{j} / \partial x_{i}\right)$. This model originated from the work of Boussinesq [76], who theorized the turbulent stresses balanced the mean strain rate. From (2.33), the eddy viscosity is related to the Reynolds stress tensor and can be written explicitly as

$$
-\overline{u_{i}^{\prime} u_{j}^{\prime}}=\nu_{T}\left(\frac{\partial U_{i}}{\partial x_{j}}+\frac{\partial U_{j}}{\partial x_{i}}-\frac{2}{3} \frac{\partial U_{k}}{\partial x_{k}} \delta_{i j}\right)-\frac{2}{3} k \delta_{i j}
$$

When evaluated over a free-shear flow similar to the bending jet, the shear component is the dominant contributor to turbulence kinetic energy. Known as the linear viscosity model, (2.34) can be rewritten for $i=1, j=2$ as

$$
-\overline{u^{\prime} v^{\prime}}=\nu_{T}\left(\frac{\partial U}{\partial y}+\frac{\partial V}{\partial x}\right)
$$

where $\nu_{T}$ is the slope of a linear fitting.

For a more dynamic prediction of $\nu_{T}$, "sub-grid scale" (SGS) turbulence models were developed to use known flow properties to converge on a solution. A variety of SGS models have been proposed to solve for $\nu_{T}$. One-equation models, such as the Smagorinsky Model [79], use grid spacing and local gradients to determine $\nu_{T}$. With the benefit of DNS data, the one-equation Spalart-Allmaras model [118] evaluates sub-grid contributions through empirical relationships. More commonly, two-equation models are used in both research and engineering applications. The 
work presented in this thesis will focus on two of them.

\subsubsection{Sub-Grid Scale Two-Equation Models}

Two-equation models have been developed to use components directly related to the turbulence present within the sub-grid. The two most prominent models, $k-\varepsilon$ and $k-\omega$, are evaluated using features of the flow that directly extracted from data. The first, $k-\varepsilon$, defines the eddy viscosity as a function of the turbulence kinetic energy, $k$, and the energy dissipation rate, $\varepsilon$,

$$
\nu_{T} \equiv C_{\mu} k^{2} / \varepsilon
$$

where $C_{\mu}$ is the modelling coefficient. The two components are determined by solving the transport equations for each component in conjunction with (2.36) [78],

$$
\begin{aligned}
\frac{\mathrm{D} \varepsilon}{\mathrm{D} t} & =\frac{1}{\rho} \frac{\partial}{\partial x_{j}}\left[\frac{\mu_{T}}{\sigma_{\varepsilon}} \frac{\partial \varepsilon}{\partial x_{j}}\right]+\frac{C_{1} \mu_{T}}{\rho} \frac{\varepsilon}{k}\left(\frac{\partial u_{i}}{\partial x_{j}}+\frac{\partial u_{j}}{\partial x_{i}}\right) \frac{\partial U_{i}}{\partial x_{j}}-C_{2} \frac{\varepsilon^{2}}{k} \\
\frac{\mathrm{D} k}{\mathrm{D} t} & =\frac{1}{\rho} \frac{\partial}{\partial x_{j}}\left[\frac{\mu_{T}}{\sigma_{\varepsilon}} \frac{\partial k}{\partial x_{j}}\right]+\frac{\mu_{T}}{\rho}\left(\frac{\partial u_{i}}{\partial x_{j}}+\frac{\partial u_{j}}{\partial x_{i}}\right)-\varepsilon
\end{aligned}
$$

There are five coefficients within this system of equations. For systems of planar jets and mixing layers, these coefficients are constant [119] and defined as

$$
C_{\mu}=0.09, \quad C_{1}=1.44, \quad C_{2}=1.92, \quad \sigma_{k}=1.0, \quad \sigma_{\varepsilon}=1.3 .
$$

Variations in $C_{\mu}$ and $C_{2}$ have been noted by Launder \& Spalding [78], Launder et al. [120] and Rodi [121] for axi-symmetric jets. Defined as a function of the centerline 
velocity, $U_{c l}$,

$$
C_{\mu}=0.09-0.04 f \quad C_{2}=1.92-\frac{f}{15} \quad f=\left|\frac{Y}{2 \Delta U}\left(\frac{\partial U_{c l}}{\partial x}-\left|\frac{\partial U_{c l}}{\partial x}\right|\right)\right|^{0.2}
$$

Similar to the $k-\varepsilon$ two equation model, the $k-\omega$ model determines the unresolved contributions by simultaneously solving the transport equations for turbulence kinetic energy and the specific dissipation rate [122]. The second term, although similar in name to $\varepsilon$, is defined as

$$
\omega=\frac{\varepsilon}{\beta^{*} k}
$$

where the closure term $\beta^{*}$ is equivalent to the closure term $C_{\mu}$, generally accepted as $\beta^{*}=C_{\mu}=0.09$

The transport equations are similar but require different closure arguments. They are defined as

$$
\begin{aligned}
& \frac{\mathrm{D} k}{\mathrm{D} t}=\frac{1}{\rho} \frac{\partial}{\partial x_{j}}\left[\left(\mu+\sigma^{*} \mu_{T}\right) \frac{\partial k}{\partial x_{j}}\right]+\frac{1}{\rho} \tau_{i j}-\beta^{*} \rho \omega k \\
& \frac{\mathrm{D} \omega}{\mathrm{D} t}=\frac{1}{\rho} \frac{\partial}{\partial x_{j}}\left[\left(\mu+\sigma \mu_{T}\right) \frac{\partial \omega}{\partial x_{j}}\right]+\frac{1}{\rho} \frac{\gamma \omega}{k} \tau_{i j}-\beta \rho \omega^{2}
\end{aligned}
$$

where

$$
\tau_{i j}=\mu_{T}\left[\frac{\partial u_{i}}{\partial x_{j}}+\frac{\partial u_{j}}{\partial x_{i}}-\frac{2}{3} \frac{\partial u_{k}}{\partial x_{k}} \delta_{i j}\right]-\frac{2}{3} \rho k \delta_{i j}
$$

\subsection{Dissipation from Modified Structure Function Method}

For many sub-grid models, the definition of the closure arguments requires the small scale contributions to be accurately represented. The dissipation, $\varepsilon$, is highly 
dependent on the scales of the data, defined as

$$
\varepsilon \equiv 2 \nu\left\langle s_{i j} s_{i j}\right\rangle
$$

where $s_{i j}$ is the fluctuating strain rate tensor defined as

$$
s_{i j}=\frac{\partial u_{i}^{\prime}}{\partial x_{j}}+\frac{\partial u_{j}^{\prime}}{\partial x_{i}} .
$$

Assuming incompressibility and applying the divergence free continuity equation,

$$
\frac{\partial u_{3}^{\prime}}{\partial x_{3}}=-\left(\frac{\partial u_{1}^{\prime}}{\partial x_{1}}-\frac{\partial u_{2}^{\prime}}{\partial x_{2}}\right)
$$

one unknown is removed. Assuming local isotropy [123], the remainder of the unresolved gradients can be related to known gradients,

$$
\begin{aligned}
\left\langle\left(\frac{\partial u_{1}^{\prime}}{\partial x_{3}}\right)^{2}\right\rangle & =\left\langle\left(\frac{\partial u_{3}^{\prime}}{\partial x_{1}}\right)^{2}\right\rangle=\left\langle\left(\frac{\partial u_{2}^{\prime}}{\partial x_{3}}\right)^{2}\right\rangle=\left\langle\left(\frac{\partial u_{3}^{\prime}}{\partial x_{2}}\right)^{2}\right\rangle \\
& =\frac{1}{2}\left\langle\left(\frac{\partial u_{1}^{\prime}}{\partial x_{2}}\right)^{2}+\left(\frac{\partial u_{2}^{\prime}}{\partial x_{1}}\right)^{2}\right\rangle \\
& \left\langle\frac{\partial u_{1}^{\prime}}{\partial x_{3}} \frac{\partial u_{3}^{\prime}}{\partial x_{1}}\right\rangle=\left\langle\frac{\partial u_{2}^{\prime}}{\partial x_{3}} \frac{\partial u_{3}^{\prime}}{\partial x_{2}}\right\rangle=\left\langle\frac{\partial u_{1}^{\prime}}{\partial x_{2}} \frac{\partial u_{2}^{\prime}}{\partial x_{1}}\right\rangle .
\end{aligned}
$$

With these relations, the dissipation can be evaluated using the known gradients.

$$
\begin{aligned}
\varepsilon_{g_{m}}=4 \nu\left\langle\left(\frac{\partial u_{1}^{\prime}}{\partial x_{1}}\right)^{2}+\right. & \left(\frac{\partial u_{2}^{\prime}}{\partial x_{2}}\right)^{2}+\left(\frac{\partial u_{3}^{\prime}}{\partial x_{1}}\right)^{2}+\left(\frac{\partial u_{3}^{\prime}}{\partial x_{2}}\right)^{2} \\
& \left.+\left(\frac{\partial u_{1}^{\prime}}{\partial x_{1}} \frac{\partial u_{2}^{\prime}}{\partial x_{2}}\right)+\frac{1}{4}\left(\frac{\partial u_{1}^{\prime}}{\partial x_{2}}+\frac{\partial u_{2}^{\prime}}{\partial x_{1}}\right)^{2}\right\rangle
\end{aligned}
$$


This is referred to as the measured gradient dissipation. Once computed, $\varepsilon_{g_{m}}$ must be corrected to avoid significant error.

On the range of scales, there is an intermediate range known as the inertial sub-range (ISR), where energy from the large scale eddies is transferred down to the smallest scales. When this region is in equilibrium, the kinetic energy of the large scales is equivalent to the dissipation of the smallest scales [34]. Kolmogorov's second theorem identified the "two-thirds law", which states the within the ISR, the dissipation is directly related to the second order structure function, $D_{L L}(\boldsymbol{x}, r)$, defined as

$$
D_{L L}(\boldsymbol{x}, r)=\overline{\left(U_{L}(\boldsymbol{x}+r, t)-U_{L}(\boldsymbol{x}, r)\right)^{2}},
$$

and scales as a function of $r^{2 / 3}$. This allows for direct measurement of the dissipation as

$$
D_{L L}(\boldsymbol{x}, r)=C_{2}\left(\operatorname{Re}_{\lambda}\right)(\varepsilon r)^{2 / 3}
$$

where $C_{2}$ is a universal constant defined as $C_{2}=2.12[124,125]$. Solving for $\varepsilon_{s_{m}}$ (the measured structure function dissipation),

$$
\varepsilon_{s_{m}}=\frac{1}{r}\left(\frac{\left\langle\left(U_{L}(\boldsymbol{x}+r, t)-U_{L}\left(U_{L}(\boldsymbol{x}, t)\right)^{2}\right\rangle\right.}{C_{2}}\right)^{3 / 2} .
$$

To determine how well this method worked, it was tested against synthetic PIV data extracted from DNS to identify an empirical relationship between the PIV grid spacing, $\Delta$, and the Kolmogorov scale, $\eta[6]$. Defining the ratio $\varepsilon_{s_{m}} / \varepsilon_{s_{c}}$ where $\varepsilon_{s_{c}}$ is the corrected structure function dissipation (from DNS data) and $\Delta / \eta$, a 


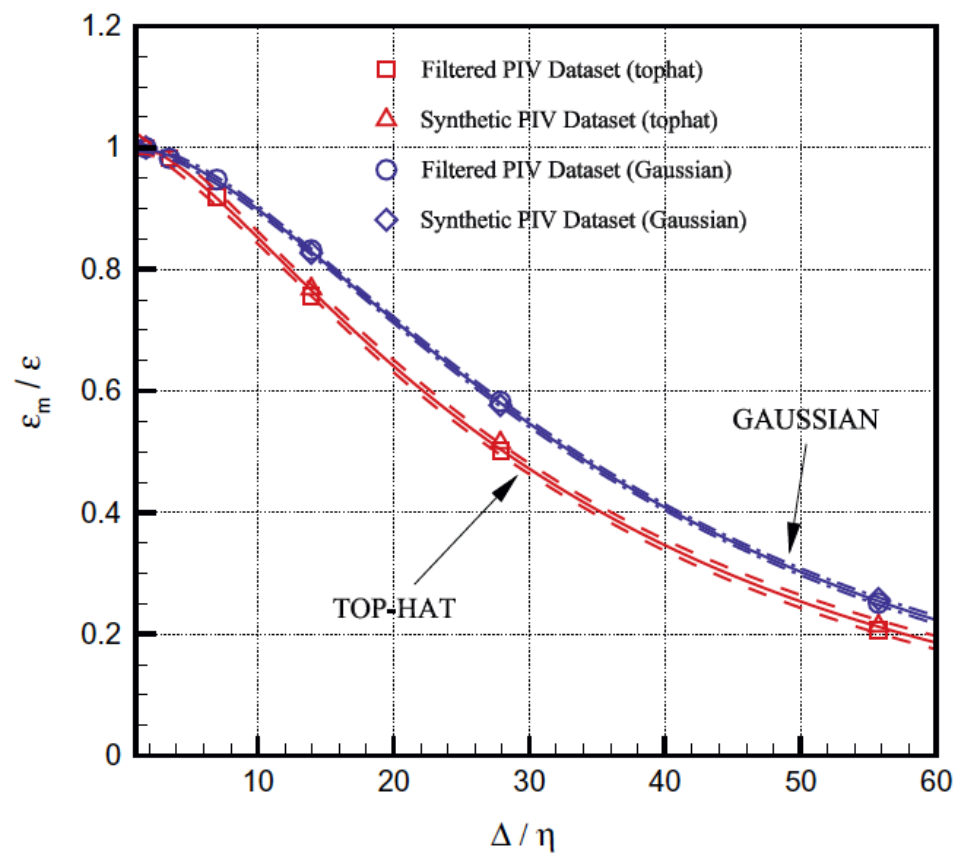

Figure 2.2: Dissipation results for data obtained using synthetic PIV data from Xu \& Chen [6, fig. 7]. The empirical fit (from (2.53)) yields the coefficients $\alpha$ and $\beta(i=1,2)$.

relationship was developed as

$$
\frac{\varepsilon_{s_{m}}}{\varepsilon_{s_{c}}}=\alpha_{1} \exp \left(\alpha_{2} \frac{\Delta}{\eta}\right)+\beta_{1} \exp \left(\beta_{2} \frac{\Delta}{\eta}\right),
$$

The values of $\alpha$ and $\beta(i=1,2)$ are determined through an empirical fit of DNS data. They are dependent on the PIV processing window filter which for this work will be Gaussian with coefficient values of

$$
\alpha_{1}=+1.2670 \quad \alpha_{2}=-0.02795 \quad \beta_{1}=-0.2737 \quad \beta_{2}=-0.1545
$$

While $\varepsilon_{s_{m}}$ and $\Delta$ are known for a given PIV data set using (2.52), $\varepsilon$ and $\eta$ are 
unknown. From the definition of the Kolmogorov length scale,

$$
\eta=\left(\nu^{3} / \varepsilon\right)^{1 / 4}
$$

and using $\varepsilon_{s_{c}}$ and $\eta$ as normalization parameters, we are left with a second equation of the form

$$
\frac{\varepsilon_{s_{m}}}{\varepsilon_{s_{c}}}=\left(\frac{\varepsilon_{s_{m}} \Delta^{4}}{\nu^{3}}\right)\left(\frac{\Delta}{\eta}\right)^{-4} .
$$

Solving (2.53) and (2.55) as a system of equations yields both $\varepsilon_{s_{c}}$ and $\eta$ for the flow.

\subsection{Critical Point Identification and Classification}

While the velocity data contains three components, the successive analysis will focus on the streamwise velocity component, $u$, and the streamwise normal component, $v$ and $w$ (for $x-y$ and $x-z$ planes respectively). From Fric \& Roshko [3], the vortical structures observed in the wake region form from instabilities in the boundary layer that are shed downstream and entrained on the lee-side of the jet. An instantaneous snapshot of the flow-field will likely not contain a velocity null in the out-of-plane component. Thus, this component is defined as negligible.

The identification of critical points (commonly using the Poincaré index to describe the topology of the velocity field), applied to turbulence by Perry \& Fairlie [126], has allowed vortices to be classified by the surrounding flow behavior. Turbulent structures have been classified as one of three topologies: nodes, saddles and foci, or a combination of the three [7]. Additionally, these features are present outside of coherent structures. Common flow features, such as stagnation and sep- 
aration points in boundary layers [127] and general flow patterns [126] where field lines intersect, are classified through critical points.

It should be noted that the approach previously mentioned will only identify first-order critical points. This means that critical points with a Poincaré index greater than 1 or less than -1 , or critical points that do not behave linearly, will not be identified. To visualize higher order critical points, a different approach, such as Scheuermann's application of Clifford algebra, must be used (see $[128,129])$. This has allowed for critical points to be identified independent of their Poincaré index.

To identify the presence of first-order critical points, Greene's Bisection Method (or topological degree theory) will be used in connection with the analytical method outlined by Gjøystdal [130]. Greene's Bisection Method was developed in response to the vorticity nulls identified in fluid dynamics[131]. Treating the critical point as the root of a function allows identification of the presence and approximation of the location. For a one dimensional function, $f(x)$, the Bisection Method identifies the presence of critical points by locating changes in sign. If one is identified over an interval, $a<x<b$, then the critical point can be located. By linearly interpolating the value halfway between the interval at $c=a+(b-a) / 2$, the critical point will exist between $c$ and the location with the opposite sign. This is then iterated until a reasonable threshold is achieved. On a more complex domain, such as a threedimensional flow-field, topological degree is used to determine the presence of a null.

From Greene [132], the topological degree, D, determines the presence of a null. Given the assumption a critical point is present at $x_{i}=x_{i 0}$, the topological degree 
is defined as

$$
\mathcal{D}=\operatorname{deg} \sum_{\sigma}\left[\operatorname{det}\left(\left.\frac{\partial J_{i j}}{\partial x_{j}}\right|_{x_{j}=x_{j 0}}\right)\right],
$$

where $J_{i j}$ is the Jacobian and $\sigma$ is the number of nulls present. Application to spatial constrained data utilizes the method of solid angles to determine the presence of a null. A volume is established (usually a box) and each face is divided along the diagonal to form two triangles. Each triangle contains a vector, $\mathbf{U}$, at each vertex. If these are connected at one vertex, a spherical triangle (a curved triangle defined on the surface of a sphere) is formed with three solid angles [130]. The area is then defined as

$$
\begin{aligned}
A=4 \tan ^{-1} & {\left[\tan \left(\frac{\theta_{1}+\theta_{2}+\theta_{3}}{4}\right) \times \tan \left(\frac{-\theta_{1}+\theta_{2}+\theta_{3}}{4}\right)\right.} \\
& \left.\times \tan \left(\frac{\theta_{1}-\theta_{2}+\theta_{3}}{4}\right) \times \tan \left(\frac{\theta_{1}+\theta_{2}-\theta_{3}}{4}\right)\right],
\end{aligned}
$$

where

$$
\theta_{1}=\cos ^{-1}\left(\frac{\mathbf{U}_{2} \cdot \mathbf{U}_{3}}{\left|\mathbf{U}_{2}\right|\left|\mathbf{U}_{3}\right|}\right), \quad \theta_{2}=\cos ^{-1}\left(\frac{\mathbf{U}_{1} \cdot \mathbf{U}_{3}}{\left|\mathbf{U}_{1}\right|\left|\mathbf{U}_{3}\right|}\right), \quad \theta_{3}=\cos ^{-1}\left(\frac{\mathbf{U}_{1} \cdot \mathbf{U}_{2}}{\left|\mathbf{U}_{1}\right|\left|\mathbf{U}_{2}\right|}\right)
$$

The solid angle is added for all triangles and the topological degree is defined as

$$
\mathcal{D}=\frac{1}{4 \pi} \sum_{i=1}^{n} A_{i}
$$

where $n$ is the number of triangles with $n=12$ for a three-dimensional space. If $D \neq 0$, a null exists within that volume. Once the presence of a null is confirmed, those cells can be saved to isolate the exact location of the critical points. From the 
thesis of Gjøystdal [130], the use of a bilinear interpolate allows for the critical point to be located will higher accuracy. For a two-dimensional velocity field, $\mathbf{U}(x, y)=$ $\left(U_{1}, U_{2}\right)$, a set of interpolation formulas are defined as

$$
\begin{aligned}
& U_{1}(x, y)=a_{1}+b_{1} x+c_{1} y+d_{1} x y \\
& U_{2}(x, y)=a_{2}+b_{2} x+c_{2} y+d_{2} x y \\
& U_{3}(x, y)=a_{3}+b_{3} x+c_{3} y+d_{3} x y
\end{aligned}
$$

where $a_{1}, b_{1}, \ldots$ are the interpolation coefficients. At the location of a critical point, $\mathbf{x}_{c r}=\left(x_{c r}, y_{c r}\right)$, the system of equations satisfy the solution

$$
U_{1}\left(x_{c r}, y_{c r}\right)=U_{2}\left(x_{c r}, y_{c r}\right)=U_{3}\left(x_{c r}, y_{c r}\right)=0
$$

Solving this system of equations with yield the location of a critical point, assuming the topological degree has identified one is present within the cell.

\subsubsection{Vortex Classification}

To further expand the description of the critical points, the topological characteristics are determined by evaluating the rate of deformation tensor $d_{i j}$, defined as

$$
d_{i j}=\left[\begin{array}{lll}
\partial_{x} u & \partial_{y} u & \partial_{z} u \\
\partial_{x} v & \partial_{y} v & \partial_{z} v \\
\partial_{x} w & \partial_{y} w & \partial_{z} w
\end{array}\right]
$$


Two problems are immediately evident. First, all out-of-plane gradients are unresolved in stereoscopic particle image velocimetry (PIV) data. Second, the vortices are not constrained to the observed plane. Shear layer vortices evolve around the edges of the jet leading to the formation of counter-rotating vortices. Within the wake region, vortices are entrained back toward the jet [3]. Therefore, to track the behavior of vortices, the out-of-plane velocity is assumed negligible. The twocomponent deformation tensor is defined as

$$
d_{i j}(x, y)=\left[\begin{array}{ll}
\partial_{x} u & \partial_{y} u \\
\partial_{x} v & \partial_{y} v
\end{array}\right] \quad \text { and } \quad d_{i j}(x, z)=\left[\begin{array}{ll}
\partial_{x} u & \partial_{z} u \\
\partial_{x} w & \partial_{z} w
\end{array}\right]
$$

A critical point, $\left(x_{c r}, y_{c r}\right)$, discovered in a velocity field is classified through the methods of Perry \& Chong [7] and Chong et al. [133]. Evaluating the deformation tensor, $d_{i j}\left(x_{c r}, y_{c r}\right)$, and projecting onto the $p-q$ domain, we assume $\lambda_{1}$ and $\lambda_{2}$ are the eigenvalues of $d_{i j}$, and are determined by solving the characteristic equation

$$
\operatorname{det}[\mathbf{A}-I]=0
$$

For the two-component deformation tensor, (2.65) is defined as

$$
\lambda^{2}+p \lambda+q=0
$$

where $p=-\operatorname{tr}\left[d_{i j}\right]$ and $q=-\operatorname{det}\left[d_{i j}\right]$. Projecting $d_{i j}$ on the $p-q$ domain categorizes a critical point into one of three categories [134] (see figure 2 in Perry \& Chong [7]):

I) The eigenvalues are real and distinct $\left(\lambda_{1} \neq \lambda_{2}\right)$. 
II) The eigenvalues are real and coincide $\left(\lambda_{1}=\lambda_{2}\right)$.

III) The eigenvalues are complex conjugates $\left(\lambda_{c r} \pm \lambda_{c i}\right)$.

Utilizing the classification methods from Furuheim \& Aansen [135], the critical points are classified according to the flow charts. For eigenvalues that fall under I or II, the surrounding flow field is classified through projection onto the $p-q$ domain, where

$$
p=-\operatorname{trace}\left[J_{i j}\right] \quad q=\operatorname{det}\left[J_{i j}\right]
$$

The relationship between $p$ and $q$ defines the flow trajectories as either a node, foci, or saddle (see Figure 2.3). The sign of $p$ indicates the stability of the node. For $p>0$, the node is stable and will entrain fluid towards the critical point. For $p<0$ the node is unstable and will repel fluid. Thus, the descriptions can provide an indication of entrainment.

When the eigenvalues are complex, the critical points are determined to be associated with vortices. For a vortex categorized under III, the square of the complex component, $\lambda_{c i}^{2}$, is defined as the swirling strength [136]. Evaluating the extent of adjacent complex roots is used to isolate vortices surrounding critical points and define the two-dimensional area [137]. While this method is useful, Hunt et al. [138] identified that this method did not account for eddies that are under strain. Within the wake region and shear layer, the complex motion indicates that this condition will be present within the flow. Thus, the connected fluid regions are identified by 


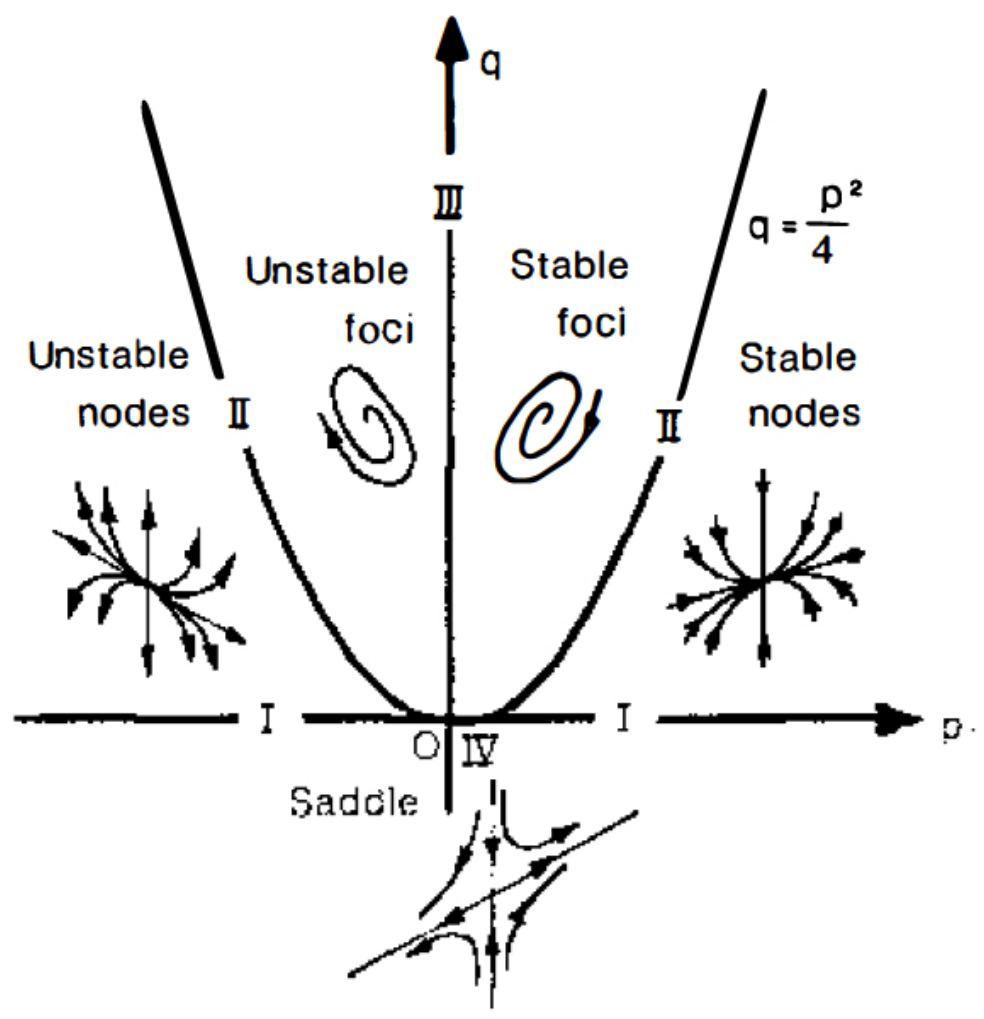

Figure 2.3: Classification of critical points projected onto the $p q$ domain from Perry \& Chong [7, fig. 2].

a positive second invariant of $d_{i j}$,

$$
Q=\frac{1}{2}\left[\left(\frac{\partial u_{i}}{\partial x_{i}}\right)^{2}-\frac{\partial u_{i}}{\partial x_{j}} \frac{\partial u_{j}}{\partial x_{i}}\right]=-\frac{1}{2} \frac{\partial u_{i}}{\partial x_{j}} \frac{\partial u_{j}}{\partial x_{i}}=\frac{1}{2}\left(\left\|\boldsymbol{\Omega}_{i j}\right\|^{2}-\left\|\boldsymbol{S}_{i j}\right\|^{2}\right)>0
$$

known as the $Q$-criterion. Tracing all connected regions provides a two-dimensional area of the vortex. 


\subsection{Proper Orthogonal Decomposition}

In highly turbulent environments, the presence of high order noise can make evaluation of Reynolds stress features difficult. For measurement systems such as PIV, removing these features can significantly improve data quality. Through a method known as proper orthogonal decomposition (POD), the dominant system features may be extracted and reordered according to their energy content. These energyordered features are captured in modes, which can be examined individually to distinguish high- from low-energy structures. They may also be used to reconstruct flow-fields ignoring high order noise. Historically, low-energy modes are most commonly omitted from POD reconstructions to highlight dominant features of a system. While POD has been used purely for its mathematical pattern recognition through Kerhunen-Loeve decomposition, Lumley proposed its application to fluid mechanics and turbulent flows [139]. The technique was then directly developed as snapshot POD by Sirovich [140]. This permitted the direct study of classical turbulence problems [141]. With improved technology such as PIV, the use of POD allowed for characterization and description of structures in turbulent flows.

Snapshot POD is regularly performed on PIV measurements, particularly those containing high energy features such as jets $[142,143,144,145]$. In the case of a round jet in cross-flow, POD analysis applied to PIV data revealed periodic variation of jet penetration in the first and second modes for both low and high velocity ratios [142]. The first two modes were also found to contain sufficient vortex-identifying information in the case of a highly turbulent swirling flow [143]. A general trend inferred from these studies is that more turbulent cases can be recreated using fewer modes, most likely due to their high-energy nature. 
For a data set from stereoscopic PIV (SPIV), a velocity field is generated of the form $\mathbf{u}\left(\mathbf{x}, \mathbf{t}^{\mathbf{n}}\right)$, where $\mathbf{u}$ contains three instantaneous velocity components, $\tilde{u}$, $\tilde{v}$, and $\tilde{w}$, $\mathbf{x}$ defines the $2 \mathrm{D}$ space $\left(x_{1}, x_{2}\right)=(x, y)$ comprised of $M$ total locations (on the snapshot) and $t^{n}$ denotes snapshot $n$ with $N$ total snapshots. Analysis is focused on the fluctuating fields, extracted through Reynolds decomposition, for three components of the velocity, the radial $u_{r}^{\prime}$, tangential $v_{r}^{\prime}$, and out-of-plane $w_{r}^{\prime}$.

Once the desired data is chosen, these flow fields are reshaped into a threedimensional $3 M \times N$ matrix, signified by $\boldsymbol{U}$ where

$$
\boldsymbol{U}=\left[\begin{array}{llll}
\boldsymbol{u}^{1} & \boldsymbol{u}^{2} & \cdots & \boldsymbol{u}^{N}
\end{array}\right]
$$

A function $\boldsymbol{\phi}$ is sought to maximize the normalized inner product of $\boldsymbol{U}$ and $\phi$,

$$
\frac{\left\langle(\boldsymbol{U}, \phi)^{2}\right\rangle}{\|\phi\|^{2}}
$$

where $(f, g)$ represents an inner product and $\langle f\rangle$ represents a mean. This occurs when $\phi$ is an eigenfunction of the two-point correlation tensor of $\mathbf{U}$ :

$$
\boldsymbol{R}\left(\boldsymbol{x}, \boldsymbol{x}^{\prime}\right)=\frac{1}{N} \sum_{n=1}^{N} \boldsymbol{U}\left(\boldsymbol{x}, t^{n}\right) \boldsymbol{U}^{T}\left(\boldsymbol{x}^{\prime}, t^{n}\right),
$$

where $\boldsymbol{x}^{\prime}$ refers to another spatial coordinate in the domain. The eigenvectors and corresponding eigenvalues are then found by

$$
\int_{\mathcal{D}} \boldsymbol{R}\left(\boldsymbol{x}, \boldsymbol{x}^{\prime}\right) \phi\left(\boldsymbol{x}^{\prime}\right) \mathrm{d} \boldsymbol{x}^{\prime}=\lambda \phi(\boldsymbol{x})
$$


where $\mathcal{D}$ is the spatial domain and $\lambda$ is a diagonal matrix of the eigenvalues of $\phi$. Once normalized, these eigenvectors $\phi$ and eigenvalues $\lambda$ act as a basis for POD analysis.

The eigenvalues along the diagonal matrix $\lambda$ are collected and sorted in descending order in a vector array. These parameters provide the energy for each given eigenvector. The modes individually reveal the energy-based development of the flow field. Because of this organization, the most energetic modes may then be selected to reconstruct the flow field. The percent energy that each of these modes holds is found by dividing each element of the eigenvalue vector array by the sum of all eigenvalues. The percent energy versus the modes reveals the number of modes necessary to reconstruct the field using a base percentage of the total energy. To reconstruct the flow fields, POD coefficients associated with each mode $a_{i}$ are calculated by re-projecting the velocity field $U$ back onto the basis of POD modes $\phi$ and integrating over the domain $\mathcal{D}$

$$
a_{i}\left(t^{n}\right)=\int_{\mathcal{D}} \boldsymbol{U}\left(\boldsymbol{x}, t^{n}\right) \phi^{(i)}(\boldsymbol{x}) \mathrm{d} \boldsymbol{x}
$$

Then, these coefficients are multiplied by their respective mode and summed

$$
\boldsymbol{U}\left(\boldsymbol{x}, t^{n}\right)=\sum_{i=1}^{N} a_{i}\left(t^{n}\right) \phi^{(i)}(\boldsymbol{x})
$$

By defining a cutoff mode, $N_{r}$, for the reconstruction, background noise and irrelevant flow features may be ignored. 


\subsection{Auto-correlation of Coherent Structures}

To determine the influence of inflow velocity and turbulence intensity on the creation of large coherent structures, two-point correlations of velocity fluctuations were employed. For two fluctuating components, $\phi_{i}$ and $\psi_{j}$, a statistical comparison between two locations can be made. Removing time dependence, the spatial correlation coefficient, $R_{i j}$, is defined as

$$
R_{i j}\left(\boldsymbol{x}_{\boldsymbol{r}}, \boldsymbol{x}\right)=\frac{\left\langle\phi_{i}\left(\boldsymbol{x}_{\boldsymbol{r}}\right) \psi_{j}(\boldsymbol{x})\right\rangle}{\sqrt{\left\langle\phi_{i}^{2}\left(\boldsymbol{x}_{\boldsymbol{r}}\right)\right\rangle} \sqrt{\left\langle\psi_{j}^{2}(\boldsymbol{x})\right\rangle}}
$$

where $\boldsymbol{x}_{\boldsymbol{r}}$ a local reference location defined as $\boldsymbol{x}_{\boldsymbol{r}}=\left(x_{r}, y_{r}\right)$. This can be approached in two ways, by evaluating the size of coherent structures, and comparing the correlation field at similar locations over two velocity field. Integration of the profile defines the size of the largest features, a similar method to the integral length scale. The second approach compares different cases to identify similar turbulent features in the the flow. For example, within the shear layer of the jet, comparisons between high and low cross-flow velocity provides a statistical comparison of the extent of similar coherent structures in radically different conditions. 


\section{Chapter 3}

\section{Experimental Setup}

This section outlines the procedures, protocols and experimental conditions that have been designed to constrain the jet to allow for the examination of turbulent features that are primarily present in the near-field. It will begin with an overview of the equipment and facilities at Portland State University, and will describe how the Wind Energy and Turbulence Lab provided the flexibility to generate reliable and comprehensive data specific to this research (Section 3.1). Section 3.2 provides details for data collection and procedures for centerline planes, Section 3.3 for wake slice planes and Section 3.4 for variable density plumes.

\subsection{Facilities}

The experiments were performed at Portland State University in a closed-circuit wind tunnel with a test section length of $5 \mathrm{~m}$ and a cross section of $0.8 \mathrm{~m} \times 1.2 \mathrm{~m}$ (shown in Figure 3.1). The free-stream wind speed has an adjustable working range of $2 \mathrm{~m} / \mathrm{s}$ to $20 \mathrm{~m} / \mathrm{s}$ in the test section. To create turbulence in the test section, an active grid system was used similar to Makita \& Sassa [114]. It consisted of an array of $10 \mathrm{~cm}$ square winglets attached to 6 horizontal and 7 vertical rods, each independently controlled by servo motors that can rotate clockwise or counterclockwise at 
a set rotational speed through a LabView control panel. The rods could be fixed to create a passive grid system, restricting the test section to under $5 \%$ turbulence intensity [103]. To create homogeneous and quasi-isotropic turbulence, the motors were allowed to run at random rotational speeds permitting up to $\sim 25 \%$ turbulence intensity in the test section.

The jet was inserted through the floor perpendicular to the cross-flow as shown in Figure 3.1 and aligned with a digital level to confirm the angle was $90.0^{\circ} \pm<0.1^{\circ}$ to the tunnel floor. This was later confirmed through PIV analysis of the jet at the floor to identify any out-of-plane or downstream motion along the centerline. To guarantee uniform turbulence within the test section, the jet was located $\sim 2.85 \mathrm{~m}$ downstream of the active grid. Compressed air from the laboratory facility was fed through a pressure regulator and allowed to mix with a seeding line for $\sim 0.85 \mathrm{~m}$. The velocity of the jet exiting into the test section was indicated with a pressure gauge and then verified through PIV. The jet apparatus (shown schematically in Figure 3.2) consisted of several interchangeable segments. The exit inner diameter $(D=9.525 \mathrm{~mm}(0.375 \mathrm{in})$.$) was constant through all experiments.$

Stereoscopic particle image velocimetry (SPIV) data was collected within one window focused on the exit of the jet to capture the near-field interaction. For the centerline plane, the two cameras placed outside of the tunnel were focused on the exit of the jet with an interior angle of $2 \theta \approx 40^{\circ}$. For the slice planes, the window was focused on planes parallel to the floor spaced vertically in increments of one jet diameter $(1 D)$.

The cameras were calibrated to a standard Type 33 plate to provide the 2D window with a region of out-of-plane spatial resolution. The laser was first projected 


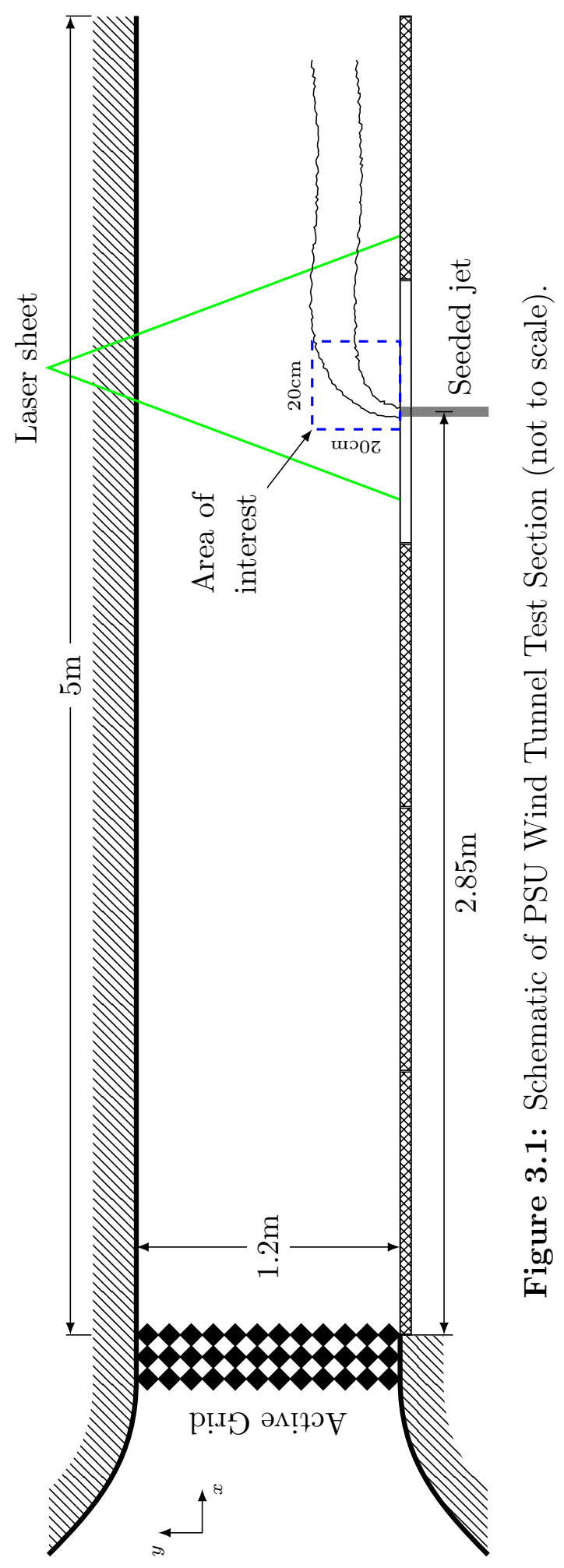




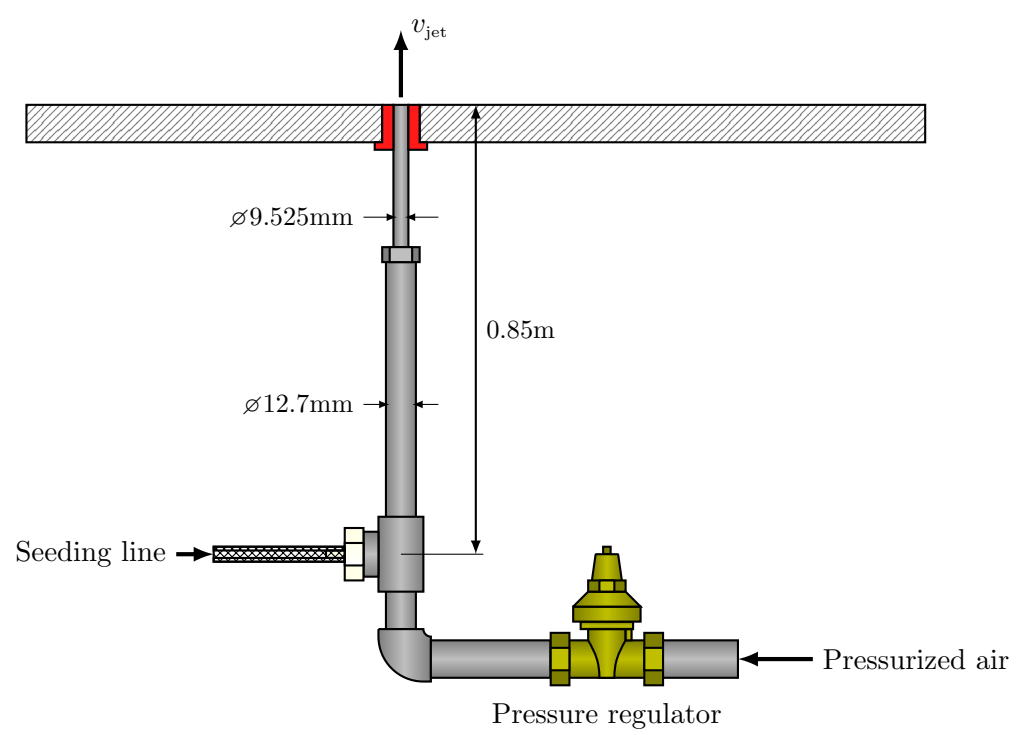

Figure 3.2: Schematic of jet system supported by frame (not shown) to hold level and flush with the test section floor. All pipe diameters refer to inner diameter measurement.

on the floor without the sheet optics to align with the center of the tunnel. The optics were then introduced and the laser sheet was focused on the target. The thickness was measured as $<2 \mathrm{~mm}$ at the thickest location. Through the PIV software, both cameras were first focused on the plate and adjusted until a centered difference of $<1$ pixels was achieved. Self-correction was repeated at decreasing interrogation windows from $128 \times 128$ to $16 \times 16$ pixels.

To ensure convergence to a mean, 2500 SPIV image sets were collected for the low turbulence cases and 3000 sets were collected for the high turbulent cases. The free-stream velocity measurements (the jet was absent) converged by 500 snapshots for low turbulence and 800 for high turbulence. Due to the increased turbulence of the jet, the number of required snapshots was increased and convergence was confirmed after testing. This also confirmed the absence of secondary flows within 

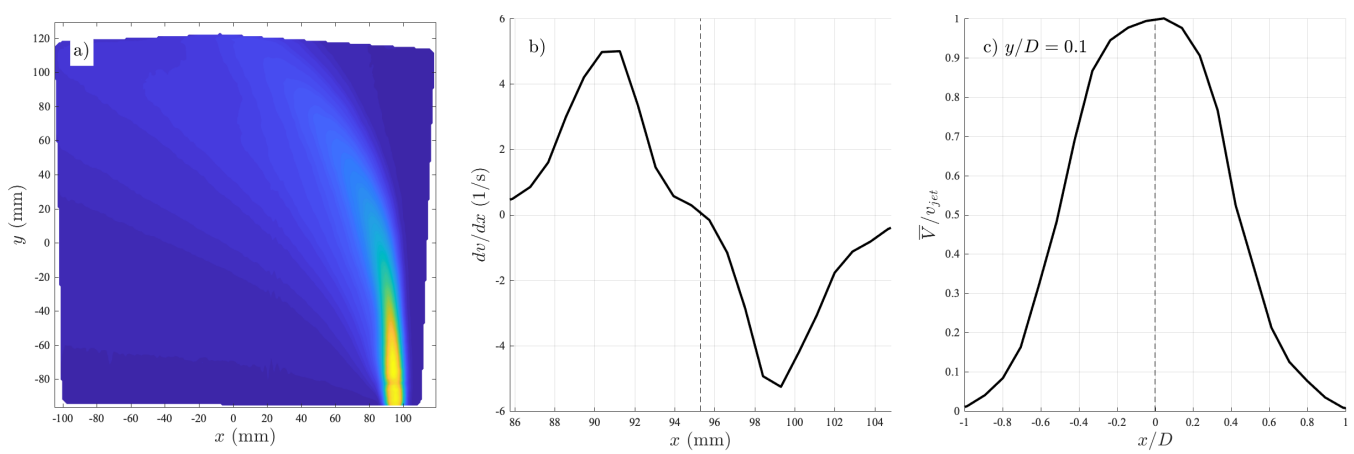

Figure 3.3: Process of identifying the center of the jet using a) raw PIV $\bar{V}$ data (before post-processing to reverse $x$ direction), and b) the slope of the velocity profile. This produces c), the normalized profile on a centered $x$-axis.

the test section.

The resulting measurement window was approximately $0.2 \mathrm{~m} \times 0.2 \mathrm{~m}$. Raw images were processed into vector fields using a multi-pass FFT based correlation algorithm. The size of the interrogation windows was reduced for each iteration (twice at $32 \times 32$ pixels and twice at $16 \times 16$ pixels), providing a vector resolution of approximately $0.1 \mathrm{~mm}$. The uncertainty calculation from DaVis PIV software utilized the methods of Wieneke[146], and did not exceed 5\%.

\subsection{Center-plane Slice Cases $(x-y$ plane $)$}

Quantification of the inflow was performed after collection through open tunnel PIV samples of 500 snapshots for the low and 800 for the high turbulent cases. Experimental data was taken at seven inflow speeds for passive grid and six for active grid (see Table 3.1). The pressure regulator was adjusted by hand and locked in place. The dynamic pressure was then measured via a pitot probe at the exit, allowing for the computations of the mean velocity. 


\begin{tabular}{|c|c|c|c|c|}
\hline & $u_{\infty}(\mathrm{m} / \mathrm{s})$ & $v_{\text {jet }}(\mathrm{m} / \mathrm{s})$ & $R_{v}$ & $\xi_{*}^{F F}$ \\
\hline \multirow{7}{*}{ 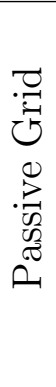 } & 1.86 & 26.0 & 13.97 & 58.55 \\
\hline & 2.68 & 26.0 & 9.71 & 28.28 \\
\hline & 3.45 & 26.0 & 7.53 & 17.01 \\
\hline & 4.30 & 26.0 & 6.05 & 10.98 \\
\hline & 5.10 & 26.0 & 5.09 & 7.77 \\
\hline & 5.94 & 26.0 & 4.38 & 5.76 \\
\hline & 6.81 & 26.0 & 3.82 & 4.38 \\
\hline \multirow{6}{*}{ 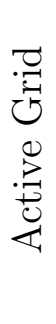 } & 2.08 & 26.0 & 12.52 & 47.03 \\
\hline & 2.45 & 26.0 & 10.62 & 33.84 \\
\hline & 2.93 & 26.0 & 8.89 & 23.71 \\
\hline & 3.28 & 26.0 & 7.92 & 18.82 \\
\hline & 3.67 & 26.0 & 7.09 & 15.08 \\
\hline & 4.42 & 26.0 & 5.88 & 10.37 \\
\hline
\end{tabular}

Table 3.1: Experimental data for the jet including the inflow velocity, $u_{\infty}$, the jet exit velocity, $v_{\text {jet }}$, Jet-to-cross-flow velocity ratios, $R_{v}=v_{\text {jet }} / u_{\infty}$, and computed far-field location, $\xi_{*}^{F F}=0.3 R_{v}^{2}$. Jet velocity was kept constant $\left(v_{\text {jet }}=\right.$ $26.0 \mathrm{~m} / \mathrm{s}$ ) and the length scale was normalized relative to the jet diameter.

The velocity data was used to identify the origin of the jet during each experiment. As shown in Figure 3.3, the velocity profile was extracted close to the exit $(y / D=0.1)$. The slope of the velocity profile was computed and the root was defined as the center. A log-log velocity profile with a turbulence intensity $\sim 10 \%$ along the centerline was observed. The centerline velocity of the jet was kept constant at $v_{\text {jet }}=26.0 \mathrm{~m} / \mathrm{s}\left(\operatorname{Re}_{D} \sim 16,700\right)$ to develop the velocity ratio of the jet, $R_{v}=v_{\text {jet }} / u_{\infty}$, where $u_{\infty}$ is the cross-flow velocity. The ratios are within the range evaluated by Smith \& Mungal [31], allowing study of the scaling parameter, $R_{v}^{2} D$. The downstream distance within the PIV window was also chosen to encompass the near-field of the jet $\left(\xi / R_{v}^{2} D \leq 0.3\right)$. 


\begin{tabular}{|c|c|c|c|c|c|}
\hline & $u_{\infty}(\mathrm{m} / \mathrm{s})$ & $u_{r m s}^{\prime} / \bar{U}$ & $L_{x} / D$ & $\operatorname{Re}_{L_{x}}$ (inflow) & $\operatorname{Re}_{D}$ (inflow) \\
\hline \multirow{7}{*}{ 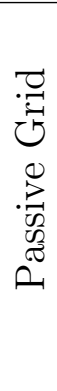 } & 1.86 & 0.0592 & 1.171 & 83.0 & 979.8 \\
\hline & 2.68 & 0.0331 & 2.301 & 131.5 & 1410.3 \\
\hline & 3.45 & 0.0317 & 2.189 & 154.1 & 1817.2 \\
\hline & 4.30 & 0.0325 & 2.185 & 196.8 & 2264.1 \\
\hline & 5.10 & 0.0341 & 2.223 & 249.0 & 2687.6 \\
\hline & 5.94 & 0.0317 & 2.333 & 282.4 & 3124.2 \\
\hline & 6.81 & 0.0368 & 1.783 & 287.2 & 3583.4 \\
\hline \multirow{6}{*}{ 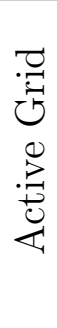 } & 2.08 & 0.1655 & 5.926 & 1311 & 1093.5 \\
\hline & 2.45 & 0.2407 & 4.455 & 1687 & 1288.7 \\
\hline & 2.93 & 0.2395 & 4.543 & 2049 & 1539.8 \\
\hline & 3.28 & 0.1792 & 6.789 & 2570 & 1728.7 \\
\hline & 3.67 & 0.1887 & 7.064 & 3144 & 1929.8 \\
\hline & 4.42 & 0.2053 & 7.318 & 4273 & 2326.3 \\
\hline
\end{tabular}

Table 3.2: Experimental data for the inflow including cross-flow velocity, $u_{\infty}$, turbulence intensity, $u_{r m s}^{\prime} / \bar{U}$, integral length scale, $L_{x} / D$, the turbulent Reynolds number, $\operatorname{Re}_{L_{x}}=u_{r m s}^{\prime} L_{x} / \nu$, and the inflow Reynolds number, $\operatorname{Re}_{D}=u_{\infty} D / \nu$ used in the current work. The length scale was normalized relative to the jet diameter.

At first, the active grid was turned off with all winglets rotated parallel to the inflow. With blockages present in the flow, this case represents a low turbulent inflow (measured to be $\sim 3-6 \%$ ) and will be referred to as "passive grid" (PG). Next, the active grid was activated using a controller that provides a random rotational speed within the range of $\pm 0.5-2$ rotations every second. Each motor was operated independently, resulting in a random speed for each rod. This case, termed "active grid" (AG), produced turbulence intensities between $16-25 \%$. The jet was placed $2.85 \mathrm{~m}$ downstream to allowed for a fully developed boundary layer profile, as seen in Figure $3.4 \mathrm{a}$ at two different velocity ratios. The corresponding turbulence intensity 

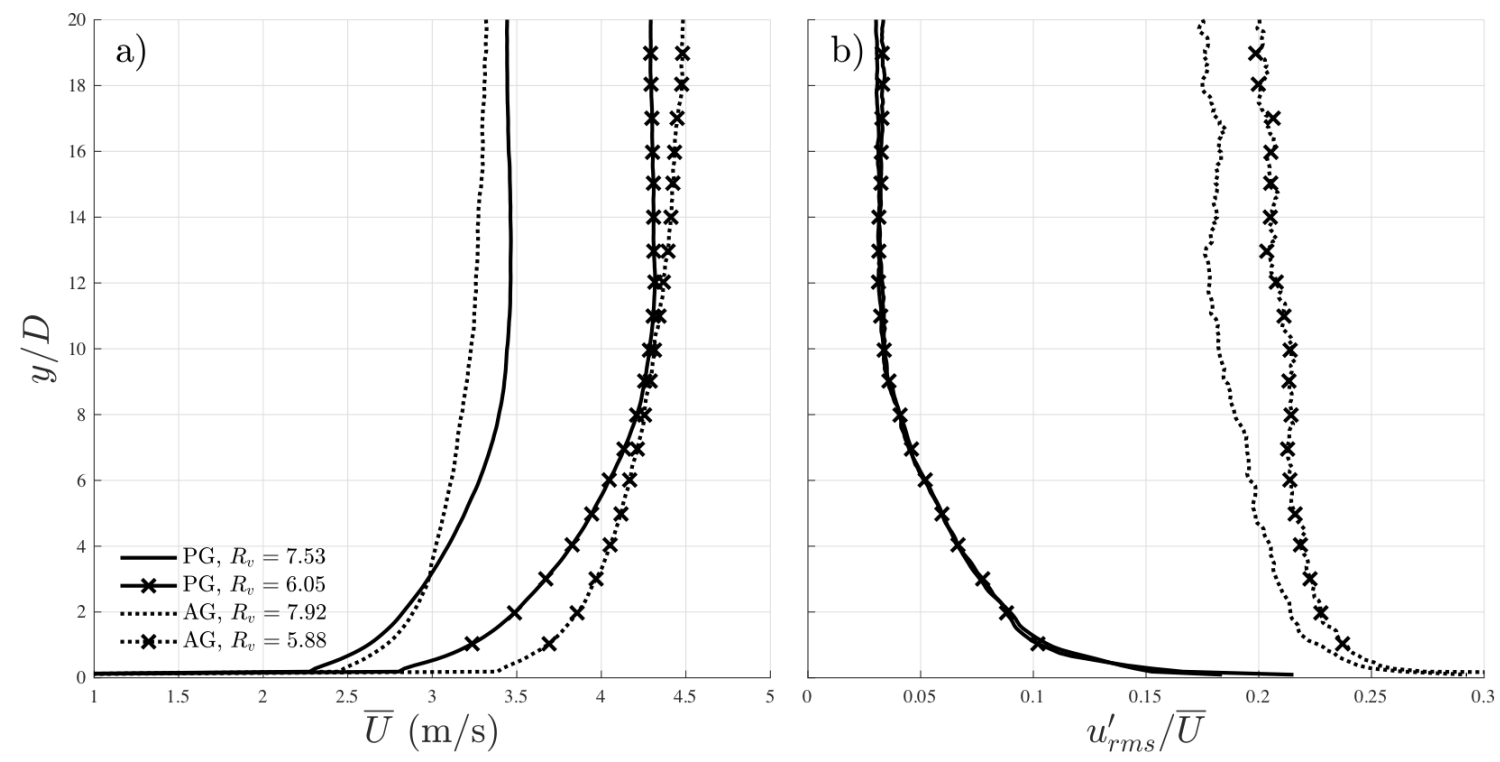

Figure 3.4: Velocity profile and turbulence intensity for two passive and two active grid cases.

profile is presented in Figure 3.4b. To further classify the turbulence, the integral length scale, $L_{x}$, was computed using a two-point correlation with the cross-flow data, as

$$
L_{x}=\int_{0}^{R} \frac{\langle\tilde{u}(x, n) \tilde{u}(x+r, n)\rangle}{\langle\tilde{u}(x, n) \tilde{u}(x, n)\rangle} d r
$$

where $n, r$, and $R$ are number of snapshots, the absolute length away from location $x$, and the length of the window, respectively. The outcome of the measurements are shown in Table 3.2. For the PG cases, the length scales are $L_{x} / D \approx 1-2.5$ while for the $\mathrm{AG}$ cases they are more than double, up to a range of $L_{x} / D \approx 4.5-7$. Because of this difference, the PG and AG cases provided an opportunity to examine the importance of turbulent eddy size in jet interactions.

To examine wake behavior, the Reynolds number of the inflow was computed as $\operatorname{Re}_{\infty}=u_{\infty} D / \nu$ assuming a constant diameter of $D=9.525 \mathrm{~mm}$. While this 
measurement does not account for the increasing jet area, it is useful to distinguish bluff body behavior around the jet. This range indicates that the wake will likely contain vortex shedding.

\subsection{Wake Slice Cases $(x-z$ plane $)$}

For additional information on the behavior of the cross-flow, experiments were developed that focused on slices parallel to the floor at increasing heights (see Figure 3.5). The cameras were aligned from above the tunnel out-of-plane of the jet $( \pm z)$. To align the various planes, a frame was built for the Type 33 plate to maintain the center relative to the jet exit. The jet was determined to be $\Delta x=50 \pm 0.5 \mathrm{~mm}$ and $\Delta z=2 \pm 0.5 \mathrm{~mm}$ from the center of the alignment plate. After collection, all planes were aligned to the jet center using this offset. The window captured data both upstream $(\sim 50 \mathrm{~mm})$ and downstream $(\sim 150 \mathrm{~mm})$ to fully encompass the wake region. Additional data was collected for each case with the jet over-seeded to yield empty data where the fluid body was present. The negligible data was counted to describe the extent of the jets fluid body at each elevation.

The experimental matrix was compiled for 3 inflow velocities with the active grid deactivated and parallel to the inflow (passive grid). Six different planes spaced at increments of $\Delta y / D=1$ were chosen to capture the developing region of the jet (before the collapse of the core) and at the collapse (for the last case). These cases were designed to investigate the effects of the inflow velocity and fluid jet body on the wake region. Due to the presence of a boundary layer, the inflow velocity approaching the jet at each height would be different. Therefore, the cases were classified by their cross-flow Reynolds number, $\operatorname{Re}_{\infty}=u_{\infty} D / \nu$, which are listed in 


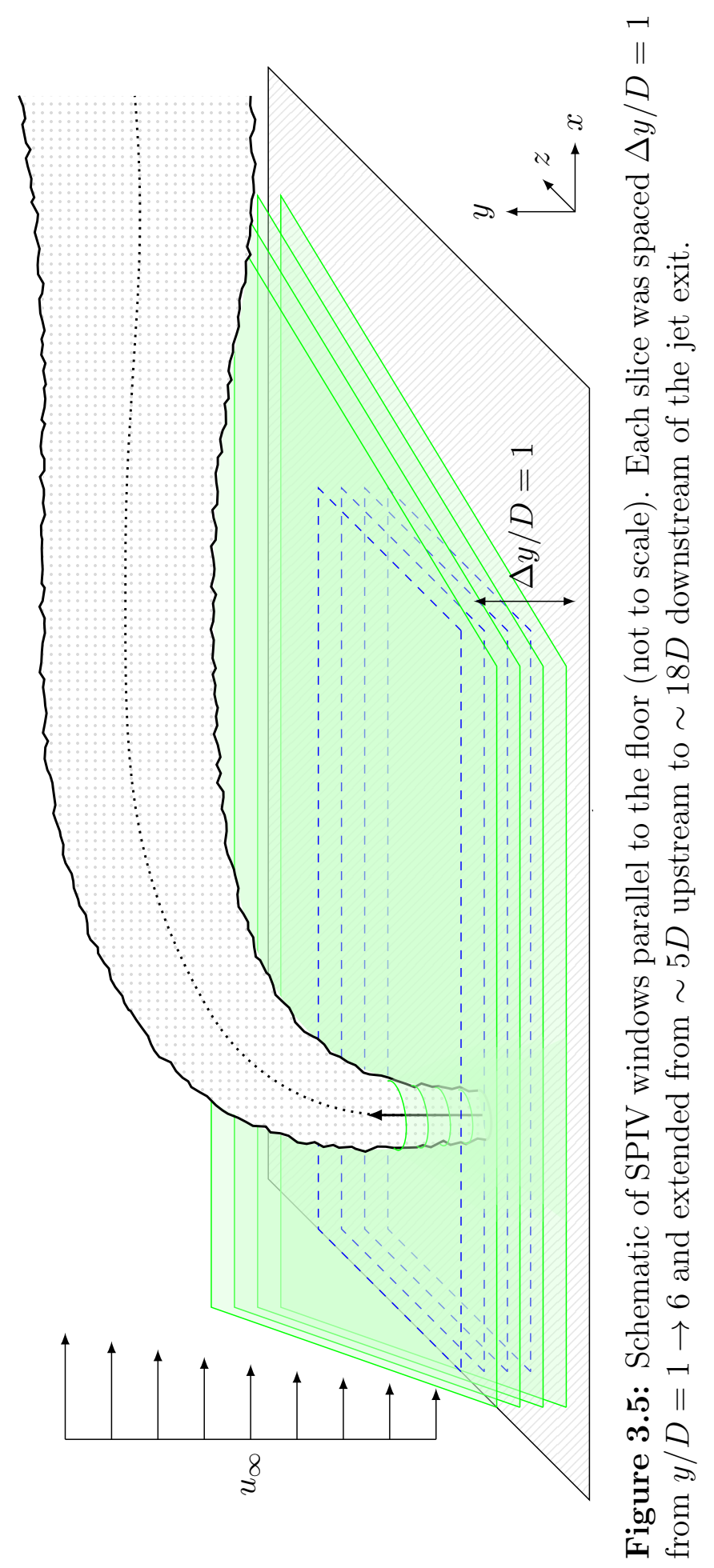




\begin{tabular}{|c|c|c|c|c|}
\hline & \multicolumn{3}{|c|}{ Inflow Cases } \\
\hline & & I & II & III \\
\hline \multirow{6}{*}{ 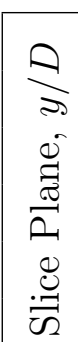 } & 1 & 858 & 1,445 & 2,164 \\
\hline & 2 & 908 & 1,585 & 2,046 \\
\hline & 3 & 921 & 1,597 & 2,191 \\
\hline & 4 & 950 & 1,628 & 2,331 \\
\hline & 5 & 969 & 1,648 & 2,425 \\
\hline & 6 & 986 & 1,715 & 2,492 \\
\hline
\end{tabular}

Table 3.3: Reynolds number of the inflow, $\operatorname{Re}_{\infty}=u_{\infty} D / \nu$, for each case.

Table 3.3. The inflow velocity was measured from 3-5D upstream of the jet.

\subsection{Variable Density Plume}

To investigate buoyant plumes, an experiment was designed focusing on two gases, argon (negatively buoyant) and helium (positively buoyant). Each gas was provided by Airgas in Portland, Oregon and were connected to a pressure regulator attached to the jet. The gases were fed at a constant pressure and the inflow velocity was obtained through PIV.

To date, some initial pilot data has been collected. The experimental matrix can be found in Table 3.4. For each case, 2500 image pairs are collected and processed to provide stereoscopic PIV data. 


\begin{tabular}{|c|c|c|c|c|}
\hline & $u_{\infty}(\mathrm{m} / \mathrm{s})$ & $v_{\text {jet }}(\mathrm{m} / \mathrm{s})$ & $R_{v}$ & $\xi_{*}^{F F}$ \\
\hline \multirow{5}{*}{$\begin{array}{l}\approx \\
0 \\
\infty 0 \\
\dot{4}\end{array}$} & 1.86 & 10.0 & 5.43 & 8.84 \\
\hline & 2.25 & 10.0 & 4.43 & 5.91 \\
\hline & 2.66 & 10.0 & 3.75 & 4.22 \\
\hline & 3.07 & 10.0 & 3.25 & 3.17 \\
\hline & 3.49 & 10.0 & 2.87 & 2.47 \\
\hline \multirow{3}{*}{ 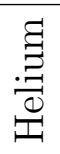 } & 1.86 & 22.0 & 11.82 & 41.97 \\
\hline & 2.66 & 22.0 & 8.26 & 20.46 \\
\hline & 3.48 & 22.0 & 6.31 & 11.95 \\
\hline
\end{tabular}

Table 3.4: Experimental pilot data for a variable density jet including the inflow velocity, $u_{\infty}$, the jet exit velocity, $v_{\text {jet }}$, jet-to-cross-flow velocity ratios, $R_{v}=v_{\text {jet }} / u_{\infty}$, and computed far-field location, $\xi_{*}^{F F}=0.3 R_{v}^{2}$. Jet velocity was kept constant $\left(v_{\text {jet }}=10.0\right.$ and $22.0 \mathrm{~m} / \mathrm{s}$ for Argon and Helium respectively) and the length scale was normalized by the jet diameter. 


\section{Chapter 4}

\section{Results}

This section presents an the analyses and additional insights gained from the data generated by the experimental design outlined in Chapter 3. The first step in this analyses included recognition and description of features, leading to the ability to note distinct changes to flow in the presence of turbulence. The results were then reoriented onto a relative flow-field to describe motions as a function of the jet

length, allowing comparisons across all cases. Next, changes due to both inflow and free-stream turbulence were quantified, which in turn informed calculations that focused on two features of the flow: the near-field wake and turbulent mixing within the jet. Finally, successive outcomes were treated through established methods of analysis to extract information on the turbulent features.

\subsection{Center-plane $(x-y)$}

From the cases outlined in Chapter 3.2, mean statistics were collected from raw images. Using software designed to provide PIV correlations, instantaneous snapshots of velocity data were generated that were cropped to focus on the specific area of interest. All velocities were non-dimensionalized by the jet exit velocity, $v_{\text {jet }}$, and represented as $u_{*}=\tilde{u} / v_{\text {jet }}, v_{*}=\tilde{v} / v_{\text {jet }}$ and $w_{*}=\tilde{w} / v_{\text {jet }}$. All lengths, including the 
spatial axes, were non-dimensionalized by the jet diameter, $D=9.525 \mathrm{~mm}$, and represented as $x_{*}=x / D, y_{*}=y / D$ and $z_{*}=z / D$. All subsequent length scales were represented in diameters. Each case was decomposed into mean and fluctuating velocity components from (2.1).

\subsubsection{Mean Flow Statistics and Reynolds Stresses}

The mean flow statistics, $\bar{U}_{*}, \bar{V}_{*}$ and $\bar{W}_{*}$, are presented in figure 4.1. All mean velocity components highlight the development of the jet and the cross-flow interaction. The PG and AG case shown possess a similar velocity ratio within the range of $7.5<R_{v}<8$.

The streamwise velocity, $\bar{U}_{*}$, shown in figures 4 .1a-b, displays four distinct behaviors of interest:

i) The negligible velocity along the leading edge of the jet;

ii) The negligible streamwise component that transitions to high streamwise velocity as the jet bends downstream;

iii) The negative (upstream) velocity on the lee-side of the jet; and

iv) The negligible velocity downstream of the negative velocity region that returns to the cross-flow velocity on the downwind side.

Along the leading edge of the jet $\left(x_{*} \approx-0.5\right)$, the streamwise velocity decreases becoming negligible near the exit indicating a stagnation point at the jet/crossflow boundary. Moving away from the floor, the velocity was small but no longer negligible while moving downstream. This indicates that at the exit, when the 


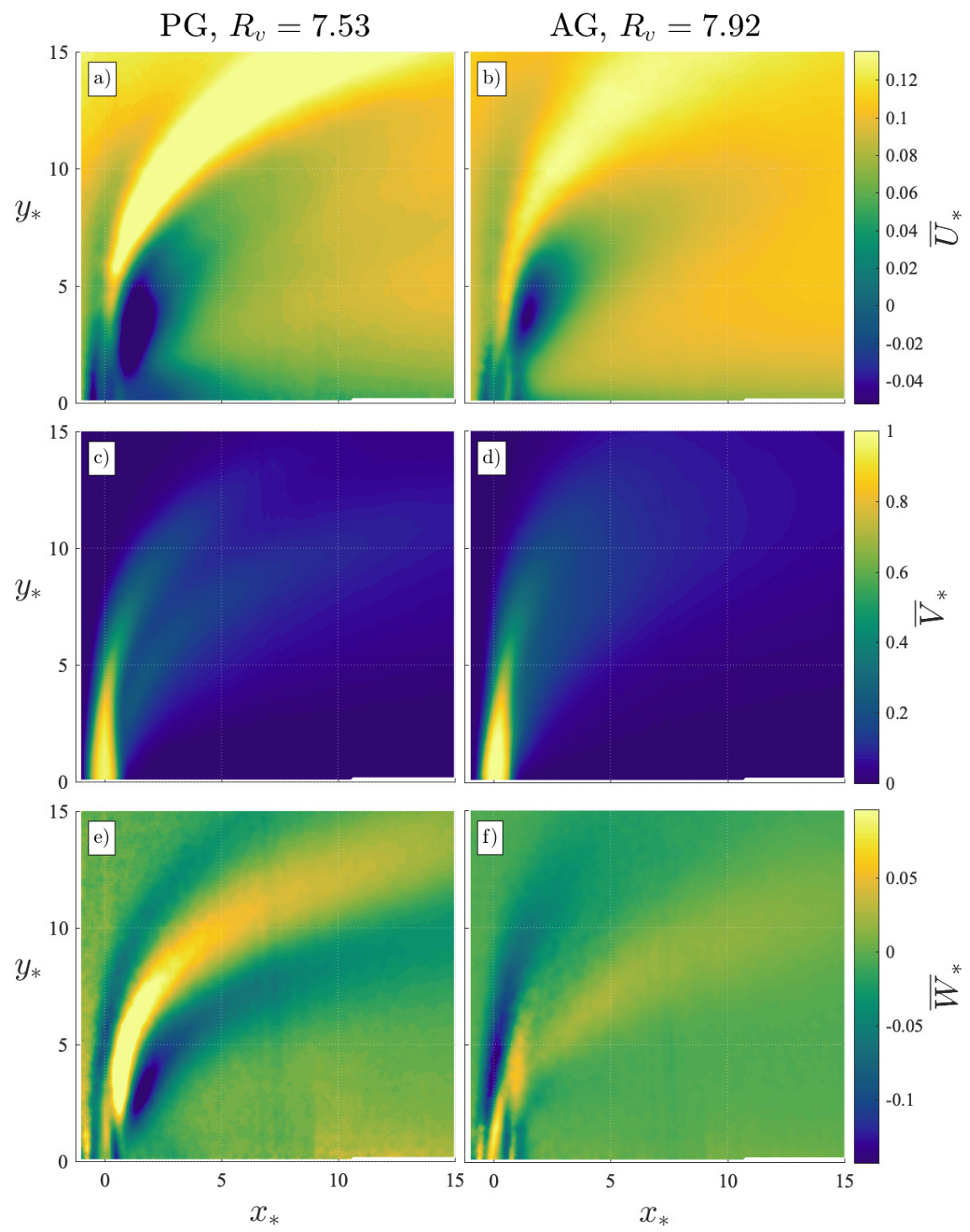

Figure 4.1: The streamwise $\bar{U}_{*}(\mathrm{a}-\mathrm{b})$, vertical $\bar{V}_{*}(\mathrm{c}-\mathrm{d})$, and out-of-plane $\bar{W}_{*}$ (e-f) mean flow statistics for one passive grid and one active grid case with similar $R_{v}$. 
shear layer of the jet was negligible, the cross-flow observes a bluff body in the flow it cannot move through. It is deflected up the leading edge or around the jet. Above this, the small magnitude of the velocity indicates an entraining surface similar to the near-field of a free-jet where stagnant flow is drawn in.

On the other side of this boundary, within the core of the jet, the negligible streamwise velocity confirms the jet was normal to the cross-flow and that the cross-flow does not initially move through the jet. Moving away from the exit, the jet begins to bend downstream and the purely vertical velocity at the exit was gradually redirected downstream. The combination of these two features identify that the cross-flow applies a body force to the jet while the shear layer entrains fluid as it is decelerated near the boundary.

With increased turbulence within the cross-flow, the interactions change. The thickness of the negligible velocity along the leading edge was compressed, and the expansion of the jet as it carried downstream increases. This is the first indication of changes in entrainment along the leading edge. For the jet to expand, the turbulence within the jet must expand into the cross-flow. In a fully turbulent environment, this process was accelerated, resulting in increased entrainment and less cross-flow deflected around the jet.

On the lee-side of the jet, the upstream motion of the flow indicates there was a wake region that was formed due to the difference in pressure from the leading edge. Cross-flow that was deflected around the jet was entrained and recirculated. At the edge of this region was a small interface of negligible streamwise velocity before the cross-flow resumes downstream movement. This was an indicator of an "unstable focus", a critical point near the exit of the jet numerically predicted by 
Sykes et al. [66], observed by Kelso et al. [59] and later corroborated by Hasselbrink \& Mungal [67], who found it to be present even in the absence of a wall boundary. Similar to the leading edge, the wake region was significantly influenced by highly turbulent inflow. For the PG case, the cross-flow does not return to the cross-flow velocity immediately downstream of the unstable focus. Along the floor, a boundary layer was formed that extends up to $y_{*} \approx 3$. From the stagnation point upstream of the jet, a separation of flow forms the horseshoe vortex system that wraps around the jet [58]. As identified in Fric \& Roshko [3], this vorticity was carried downstream as a wall vortex system within the wake region that continues to interact with the jet.

For the AG cases, this region of recirculated flow was clearly smaller. Along the floor the boundary layer thickness was reduced and the reduced streamwise velocity recovers closer to the unstable focus. Similar to the behavior of a bluff body, this wake deficit decay was dependent on the inflow velocity. Similar to the wake behavior observed by Wu \& Faeth [147], Legendre et al. [148] for spheres and Eames et al. [149] for cylinders, the wake recovery was dependent on inflow turbulence. For low inflow turbulence, the streamwise velocity deficit was still present $x_{*}=$ 15 downstream while for high turbulence, the wake deficit disappears $x_{*}=\sim 10$ downstream. Therefore, the wake region of the jet behaved similar to wake behind a rigid bluff body.

In the vertical mean velocity component, $\bar{V}_{*}$, shown in figures $4.1 \mathrm{c}$-d, the transition to a bending jet was observed. The largest velocities were found within a narrow column at the exit $\left(-0.5 \leq x_{*} \leq 0.5\right)$ which expanded and decreased in magnitude as the jet bends downstream. The jet became asymmetric downstream, 
showing a distinct boundary on the leading edge and a wider region of low velocity on the lee-side. Within the wake region, the PG cases contained a small region on increased vertical velocity that appeared to be a secondary flow under the jet. As illustrated by Cortelezzi \& Karagozian [53], as the counter-rotating vortices were formed, the cross-flow that was near the jet was drawn into the center-plane. Once drawn in, this fluid could not escape and continues to be entrained by the jet along the lee-side boundary.

When discussing the out-of-plane component, $\bar{W}_{*}$, (Figure 4.1e-f) it should first be noted that there should have been negligible out-of-plane movement. From Cortelezzi \& Karagozian [53] the presence of counter-rotating vortices within the jet and wake region (observed on the $x-z$ plane) indicated that out-of-plane velocity would be present everywhere besides the centerline of the jet. Although the laser sheet was centered on the jet, its thickness $\left(\Delta z_{*} \approx 0.2\right)$ would capture particles within the counter-rotating vortices or the entrained cross-flow within the wake region. Therefore this velocity component was used to describe the mean movement of particles into and out of the center-plane, not the mean velocity at $z_{*}=0$. Additionally, the out-of-plane velocity indicates there was asymmetry to the flow further downstream. For rotating bluff bodies such as those studied in Diaz et al. [150], the wake profile became asymmetric when the body was not solid. For a highly vortical body in the flow, a slight asymmetry would be expected. Therefore, the formation of the counter-rotating vortices are not exactly on the center-plane and skewed to one side of the jet.

For the passive grid case seen in Figure 4.1e, there was out-of-plane velocity in the cross-flow approaching the jet. On the lee-side, there was a large region of 
higher magnitude velocity within the wake region. This indicated that the cross-flow moves around the jet and was entrained in the wake region. When the cross-flow turbulence intensity was increased, the out-of-plane component on the leading edge increased in magnitude, as seen in Figure 4.1f. This region spreads along the length of the jet. The flow entrained on the lee-side dramatically decreased in magnitude, becoming almost negligible downstream. With higher turbulence, the wake region and resulting counter-rotating vortices were reduced in extent.

While the velocity components display the interaction between the jet and crossflow, they did not provide sufficient information to distinguish their boundaries. For this, the fluctuating velocity components, $u^{\prime}, v^{\prime}$ and $w^{\prime}$, were used to compute the Reynolds stresses, with two normal and one shear component presented in Figure 4.2. Although the turbulence kinetic energy could also be computed, only the individual normal stresses were shown to better distinguish the orientation of the fluctuations.

Examining the streamwise normal stress component, $\overline{u^{\prime} u^{\prime}}$ (presented in the figures $4.2 \mathrm{a}-\mathrm{b})$, the streamwise fluctuations were small near the exit and present only at the edges of the jet. This indicated that the shear layer was beginning to form here. Moving up $\left(+y_{*}\right)$ from the exit, the stresses increased in magnitude as the shear layer expands inward towards the center of the jet. Once the shear layers converge, the stresses reach a maximum preferentially located on the lee-side of the jet. Since the jet initially did not have a streamwise component, the location denoted where the full width of the jet began to bend downstream with the cross-flow. Continuing along the path of the jet, the magnitude began to decrease and expand while leaving a distinct boundary on the lee-side of the jet.

The vertical Reynolds normal stress, $\overline{v^{\prime} v^{\prime}}$, shown in figures $4.2 \mathrm{c}-\mathrm{d}$, was present 


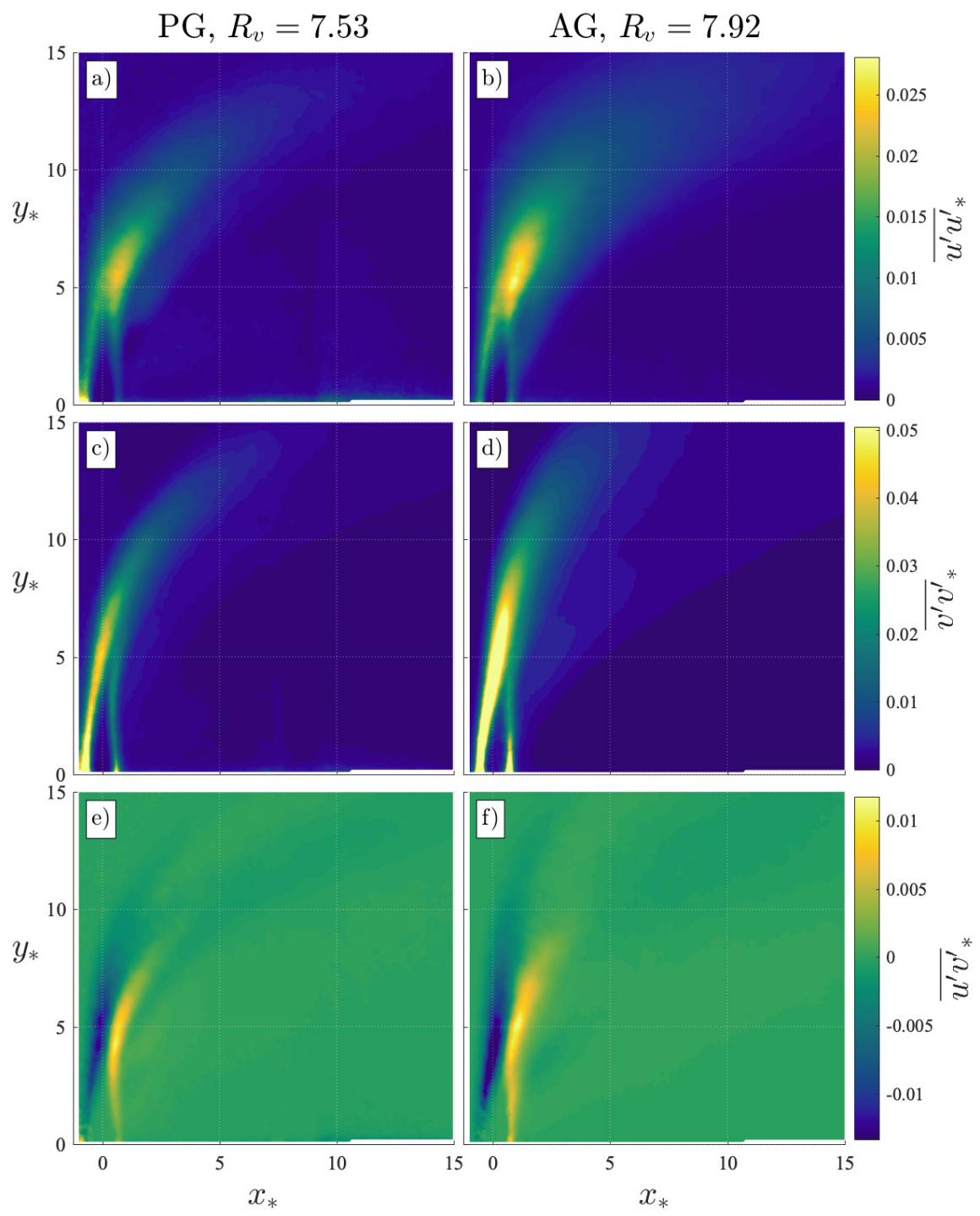

Figure 4.2: Components of Reynolds stress tensor including the streamwise (a-b) and vertical (c-d), Reynolds normal stresses, and the Reynolds shear stress (e-f) for one passive grid and one active grid case with similar $R_{v}$. 
in similar locations as $\overline{u^{\prime} u^{\prime}}{ }_{*}$. The leading edge and lee-side shear layers were present close to the exit and converge at the center of the jet to reach a maximum. Along the leading edge shear layer, the high magnitude for both normal stress components highlight the formation of shear layer vortices and the interaction with the crossflow. For the AG cases, the magnitude of the stresses was greater than the PG cases despite the constant Reynolds number of the jet. This increase in stresses along the leading edge must have been a direct result of interaction with the increased turbulence intensity.

With the Reynolds shear stress, $\overline{u^{\prime} v^{\prime}} *$ (figures $4.2 \mathrm{e}-\mathrm{f}$ ), the leading edge and leeside shear layers were the only significant contributions providing the most direct description of the boundaries of the jet. The largest shear stress occurs where these two shear layers converge, approaching each other before expanding outward again. As the turbulence intensity was increased, this expansion was more rapid.

\subsubsection{Scaling and Characterization}

To examine the jet development, the centerline was determined for each test case using the method outlined in Appendix B. Figure 4.3 displays the trajectories for all cases. The length of the centerline was computed up until the near/far-field cutoff, $\xi_{*}=0.3 R_{v}^{2}$ which has been designated as $\xi^{F F}$ and has been marked with a $\times$. Due to the difference in effective scaling parameters, the near and far-field were evaluated separately. Within the near field of the jet, the curves showed similar behavior. For all cases, the jet rises as it was pushed downstream, illustrating behavior similar to a power law. For high $R_{v}$ cases, the jet did not bend immediately as the inflow did not impart enough momentum to force it downstream. As $R_{v}$ decreases, this delay 

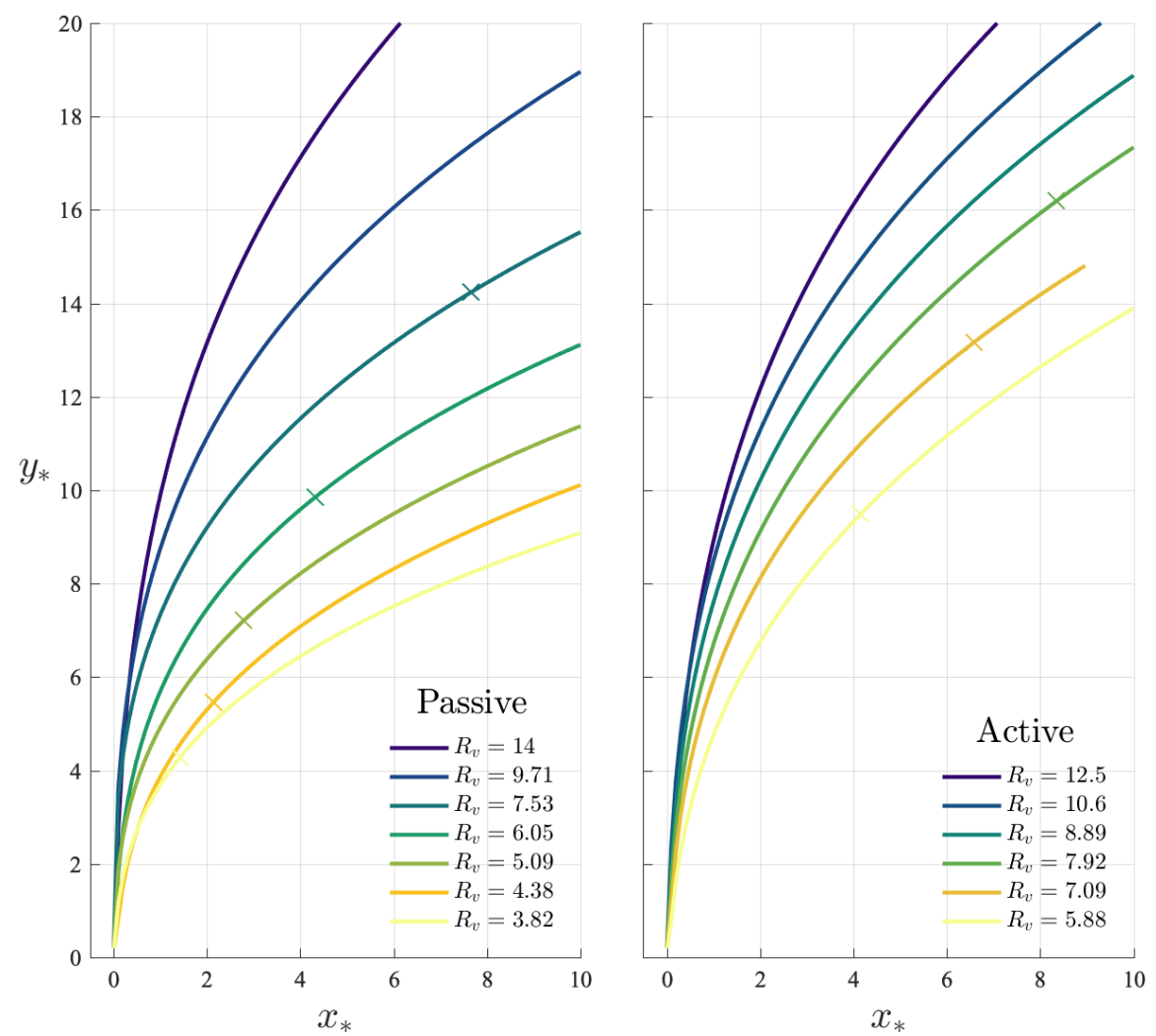

Figure 4.3: Centerline trajectories for each case, evaluated as the largest $\bar{V}$ at each height and fitted with a power law function. Near/far-field transition point $\left(\xi_{*}^{F F}=0.3 R_{v}^{2}\right)$ has been marked for each centerline $(\times)$, but exists past $x_{*}=10$ for high $R_{v}$.

disappeared and the jet bends immediately at the exit. Therefore, the centerline was defined from the location the jet was no longer vertical $\left(x_{*}>0\right)$ to $\xi^{F F}$ and was fit with a power law of the form $y=A x^{b}+c$ with coefficient $A$, exponent $b$, and offset $c$. The offset is only present on two PG cases.

Examining the fit coefficients in Figure 4.4, three different scaling parameters provided different descriptions of the jet centerline, a) the jet diameter, b) the farfield scaling, $R_{v} D$, and c) the near field scaling parameter, $R_{v}^{2} D[19]$. The spatial 

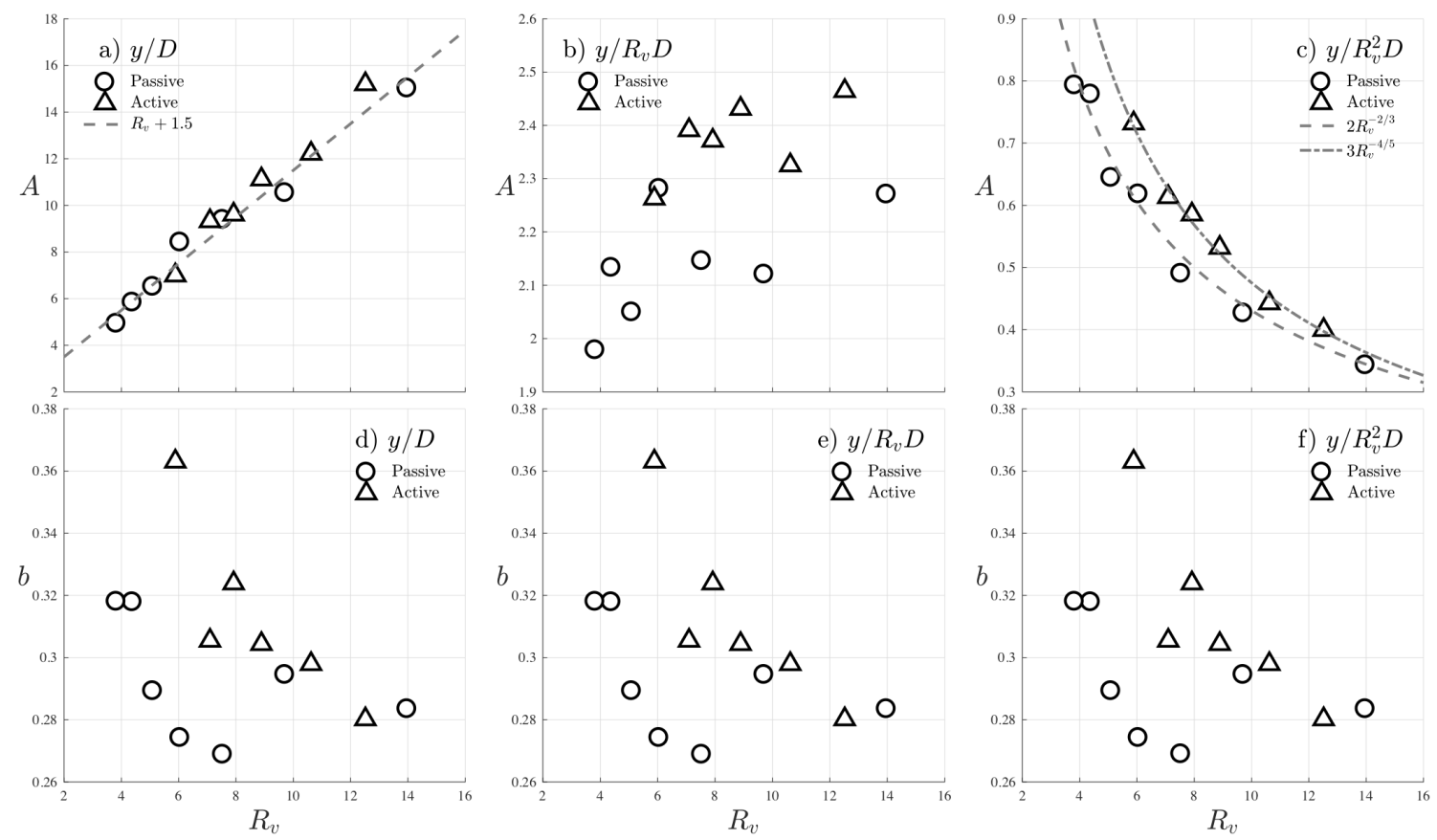

Figure 4.4: Power law coefficients for the centerline fit, $Y=A X^{b}$ where $X$ and $Y$ are scaled by $D(\mathrm{a}, \mathrm{d}), R_{v} D(\mathrm{~b}, \mathrm{e})$, and $R_{v}^{2} D(\mathrm{c}, \mathrm{f})$. Resulting converged data fits were included for $A$.

data were scaled to define the centerline as

$$
Y=A X^{b}+c \quad \text { for } \quad X=x / C, \quad Y=y / C,
$$

where $C$ was the scaling parameter. Nondimensionalizing with the diameter alone, the coefficient $A$ was independent of inflow turbulence and linearly decreased with $R_{v}$ as

$$
A=R_{v}+1.5
$$

As the inflow velocity was increased, the jet would not achieve the same final rise, so $A$ would decrease. When scaled with the far-field parameter, no convergence was 
achieved and was no longer beneficial for describing this region of the jet. Finally, the near-field parameter was unable to fully collapse the data. Two distinct curves were achieved for PG and AG, which indicated the turbulence influenced the scaling of the jet. The two curves are defined as

$$
\begin{aligned}
& A=2 R_{v}^{-2 / 3} \text { for Passive Grid } \\
& A=3 R_{v}^{-4 / 5} \text { for Active Grid }
\end{aligned}
$$

The exponent serves to describe the concavity of the jet, which was independent of scaling parameters (Figure 4.4d-f). With varying inflow velocity, the PG cases range between $b=0.25$ and 0.33 while the AG cases extend up to $b=0.36$. The exponent was largest at lower $R_{v}$ as the jet bends even more with increased inflow velocity. These values were comparable to the values discussed by Margason [18]. For increased turbulence intensity, the exponent was $\sim 15 \%$ larger, indicating the rise and concavity of the jet was influenced by turbulence.

Since each case had variations in trajectory, the original coordinate system made comparisons challenging. The axis transform defined in Appendix B allowed the flow-field to be represented using a radial distance off the centerline, $r_{*}$, and as an axial location along the centerline, $\xi_{*}$ (see Figure 2.1 in Appendix B for a detailed description). Transformation of the velocity components also provided the mean entrainment velocity, $\bar{U}_{r}$, and cross-flow velocity, $\bar{V}_{r}$, both of which were used in approximations of entrainment. Additionally, the Reynolds stresses were also computed by transforming each instantaneous snapshot and computing the relative fluctuations. In Figure 4.5, the transformed flow-fields maintained the most significant features from the mean flow and Reynolds stresses. The velocity components 

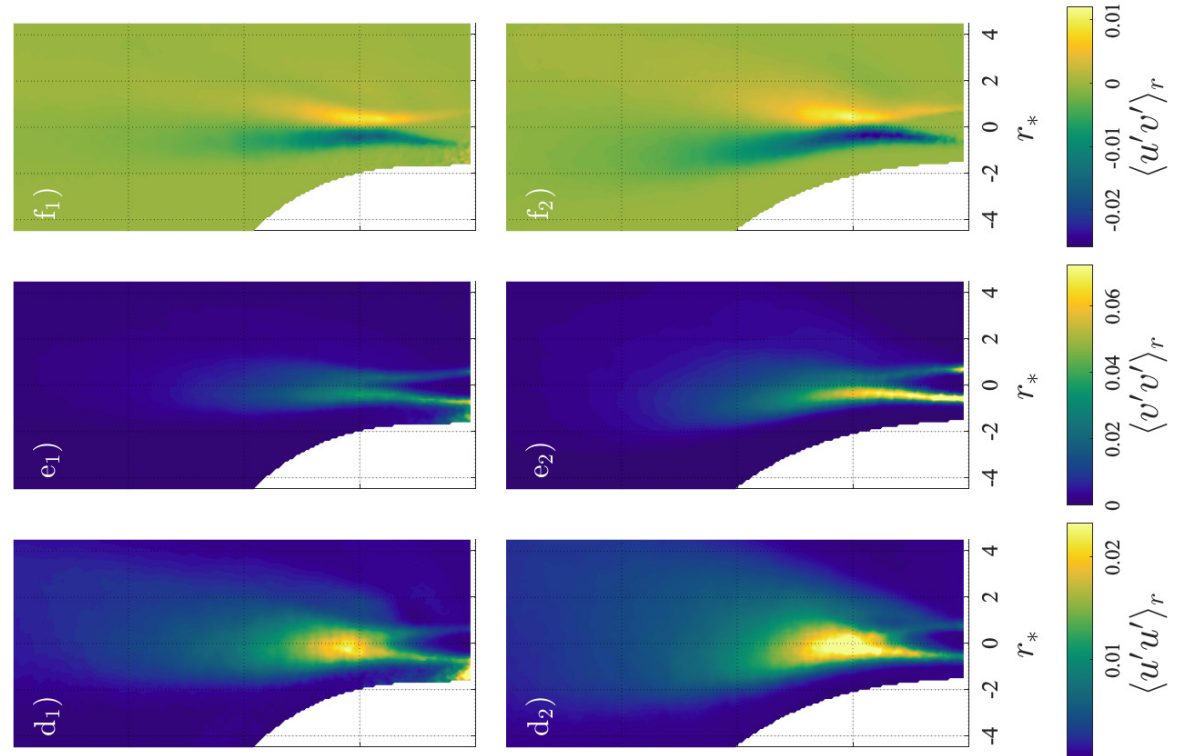

ชั
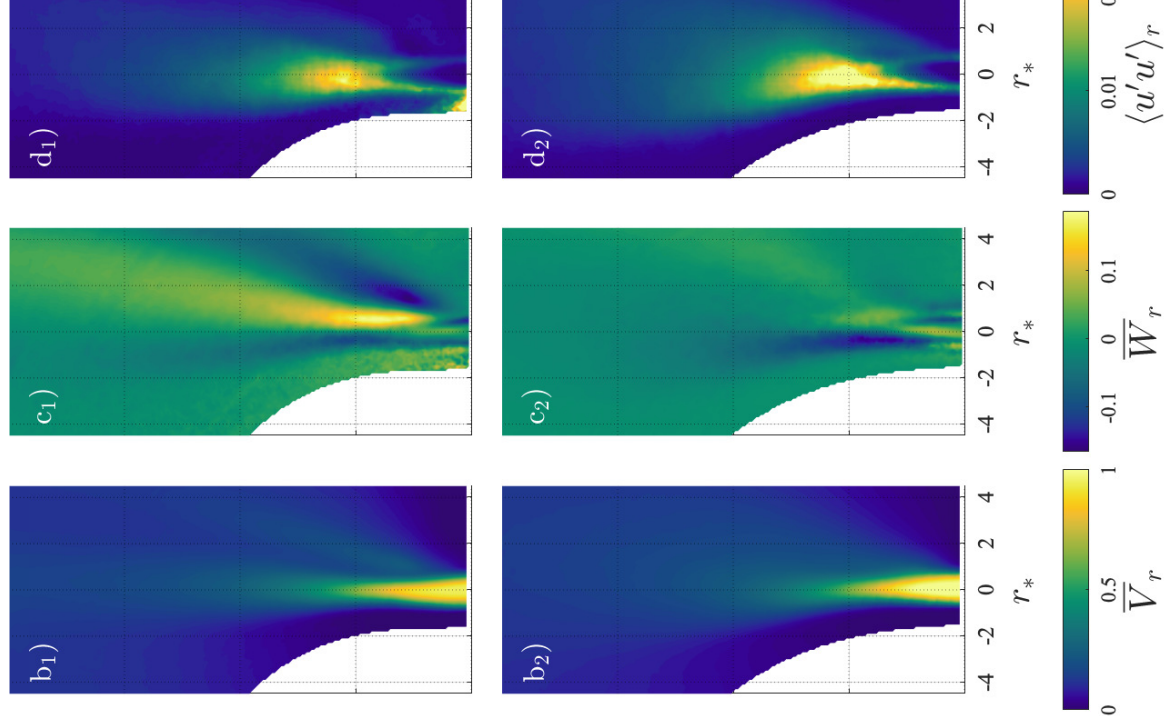

흠

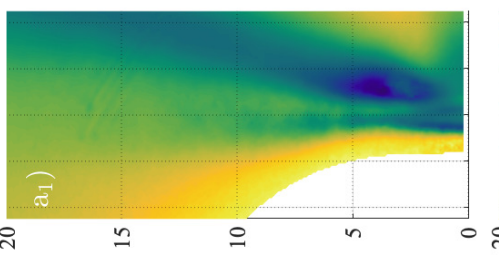

$$
R_{v}=7.53
$$

Passive Grid
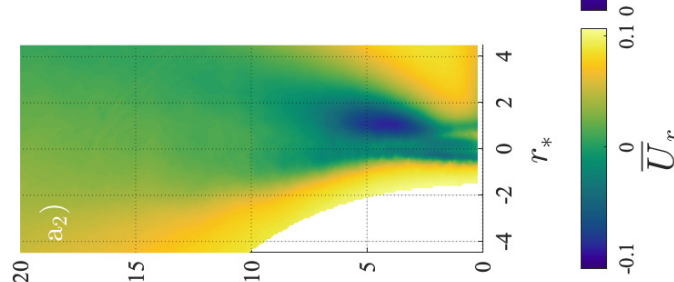

$R_{v}=7.92$

Active Grid

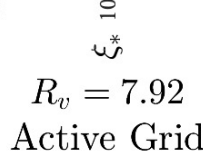


contain the lee-side wake region $\left(4.5 \mathrm{a}_{1,2}\right)$, decay of the jet velocity $\left(4.5 \mathrm{~b}_{1,2}\right)$ and the out-of-plane movement $\left(4.5 \mathrm{c}_{1,2}\right)$ was maintained. The Reynolds stresses provided a more direct description of the development of the jet. The collapse of the core coincides with the largest radial fluctuations, $\overline{u^{\prime} u^{\prime}}\left(4.5 \mathrm{~d}_{1,2}\right)$; the tangential fluctuations, $\overline{v^{\prime} v^{\prime}}$, peaked along the leading edge where shear layer vortices have been identified [50] $\left(4.5 \mathrm{e}_{1,2}\right)$; and the shear stress identified two distinct peaks within the jet that converged at the collapse of the potential core and expanded outward $\left(4.5 \mathrm{f}_{1,2}\right)$. From these components, a description of the development of the jet could be compared across the inflow conditions.

\subsubsection{Shear Layer Identification}

On the current axis, comparisons across $R_{v}$ would not describe similar flow features due to the variations in development. For all cases, a universal feature that provided similar stages of development was identified by focusing on the normal stress component $\overline{u^{\prime} u^{\prime}}$ (Figure $4.5 \mathrm{~d}_{1-2}$ ). Along the centerline, the normal stresses increased, reaching a maximum before decreasing slowly over the length of the jet (Figure 4.6). This peak, which coincided with the location of where the two shear stress peaks converged, describes the fluctuations of the jet along the centerline, or the "flapping" of the jet as it began to bend downstream. Therefore this peak was defined as the collapse of the core and the transition from strong to bending jet and referred to as $\xi^{0}$.

This location was found for all cases and occurred closer to the jet exit with increased inflow velocity. Similar to the power law coefficients, this length was scaled with the near- and far-field scaling parameters. Without scaling (Figure 4.7a) the 


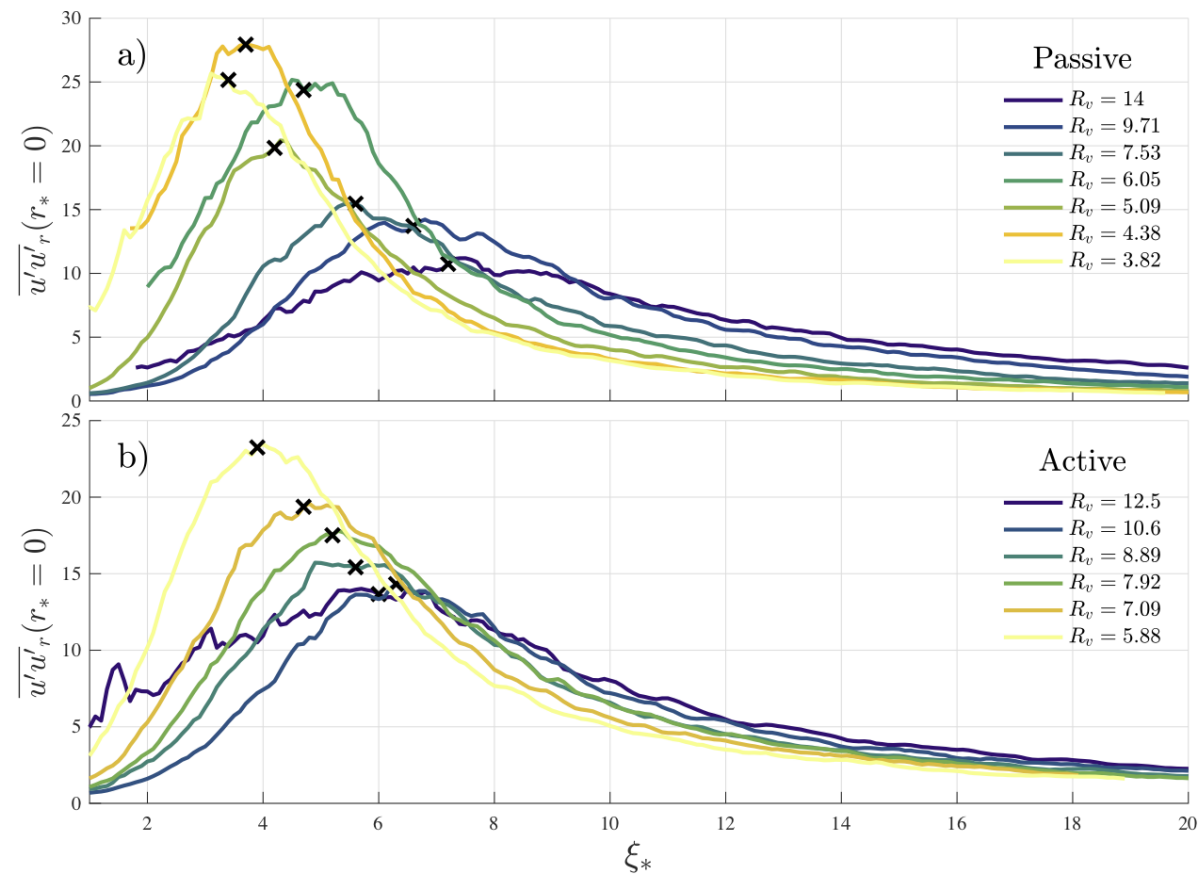

Figure 4.6: Reynolds normal stress component $\overline{u^{\prime} u^{\prime}}{ }_{r}$ along the centerline of the jet for all a) passive and b) active grid cases. The peak location (coinciding with the convergence of the normal stress peaks, marked with $\times$ ) was found to move closer to the exit as $R_{v}$ decreased.

PG and AG cases were similar but did not collapse to one curve. Excluding the largest $R_{v}$ for $\mathrm{PG}$ and $\mathrm{AG}$, a linear fit provided two similar equations,

$$
\begin{array}{ll}
\xi_{*}^{0}=0.5 R_{v}+2.65 & \text { for Passive Grid } \\
\xi_{*}^{0}=0.5 R_{v}+2.1 & \text { for Active Grid }
\end{array}
$$

A similar slope indicates these two curves could be collapsed. Because $\xi^{0}$ occured close to the exit, the far-field parameter separated the data further (Figure 4.7b). Using the near-field scaling parameter, $R_{v}^{2} D$, all the data collapsed to a power law relationship similar to the trajectory coefficient (Figure 4.7c) that followed the 

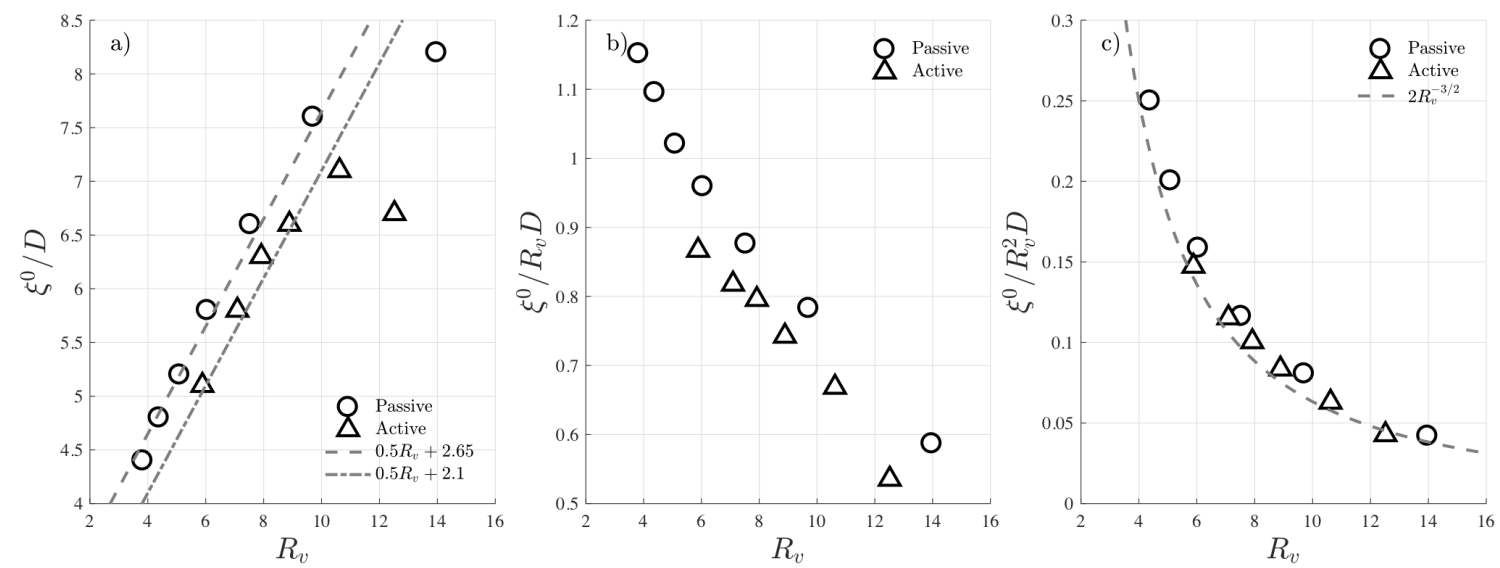

Figure 4.7: Evaluation of the scaled location of the peak radial normal stress, $\xi_{*}^{0}$. The locations were normalized by $R_{v}^{2} D$ and fitted to highlight the relationship to $R_{v}$.

relationship

$$
\xi^{0} / R_{v}^{2} D=2 R_{v}^{-3 / 2}
$$

This provided two insights into the development of the jet. First, the turbulence intensity did not affect the development of the jet, as the trajectory and collapse of the core were dependent on the velocity ratio alone. Second, the collapse of the core and transition to bending jet at $\xi^{0}$ could be defined using only the physical features and initial conditions of the jet. Since this trend was common among all cases, a unifying length scale was defined to aid further analysis.

For the purpose of defining a control volume to evaluate transport, the leading edge and lee-side shear layer edges were chosen. This allowed for both transport analysis and evaluation of entrainment parameters. For a jet in potential or irrotational flow, the edges were defined using the turbulence profile to identify when the turbulence became negligible at the outer boundary of the T/NT interface. However, the presence of free-stream turbulence suggested there was no location of negligible 

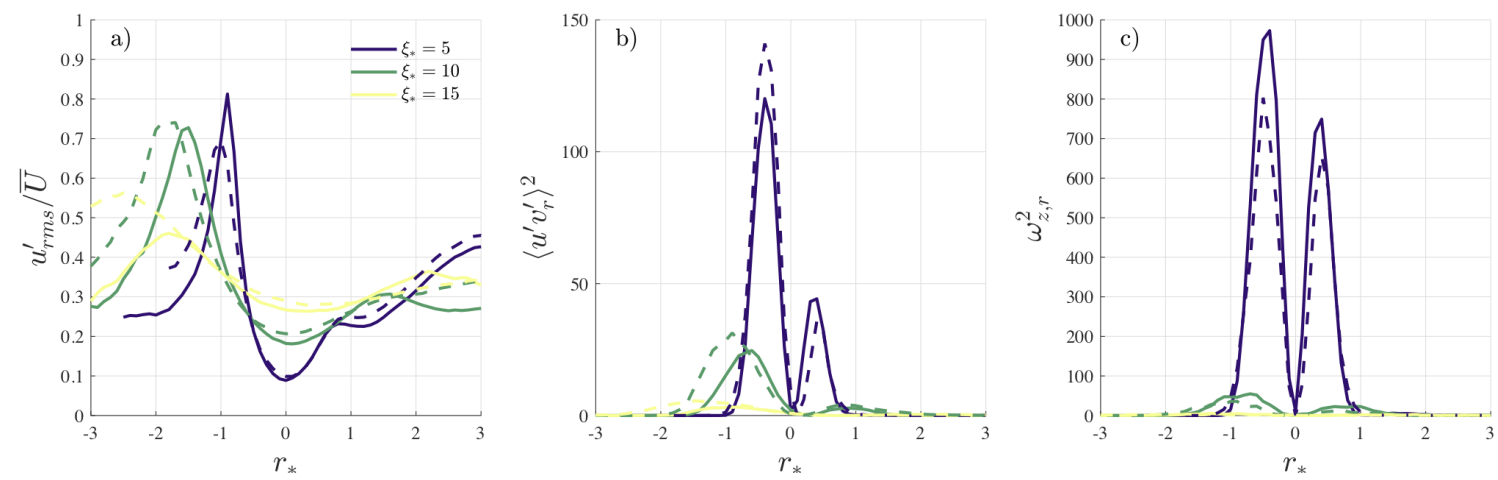

Figure 4.8: Profiles of the a) turbulence intensity, $I$, b) the squared Reynolds shear stress, $\left\langle u^{\prime} v^{\prime}\right\rangle_{r}^{2}$, and c) the squared vorticity profiles, $\left\langle\partial_{r} \bar{V}_{r}-\partial_{\xi} \bar{U}_{r}\right\rangle^{2}$ at three lengths of the jet for PG (solid) and AG (dashed).

intensity at any length along the jet (see Figure 4.8a). Moving radially outward, both sides of the jet contained no distinguishable boundary with the lee-side intensity greater in the wake region than in the jet. Between the PG and AG cases, the profiles remained similar throughout the length of the jet for a similar $R_{v}$. The AG turbulence intensity peaked further from the centerline, confirming the observation of increased expansion.

Two methods were proposed to determine the boundary of the jet. First, the jet was described through the fluctuating field, the shear stress $\left\langle u^{\prime} v^{\prime}\right\rangle_{r}$ (from Figure $4.5 \mathrm{f}_{1,2}$ ) provided a sinusoidal profile at each $\xi$ that was only present at the meeting of normal velocity components. The squared Reynolds shear stress profile (Figure 4.8b) minimized small fluctuations near the edges to isolate the shape of the jet. Second, the jet was described through the instantaneous field using the vorticity field, $\omega_{z}=\left\langle\partial_{r_{*}} \bar{V}_{r}-\partial_{\xi_{*}} \bar{U}_{r}\right\rangle$. Within the near-field of the jet, both the vorticity and its square were similar to the shear profile with two distinct peaks (Figure 4.8c).

Both methods were tested to determine the differences and the optimal fitting parameters (Figure 4.9). Details on the method used to evaluate the shear layer 


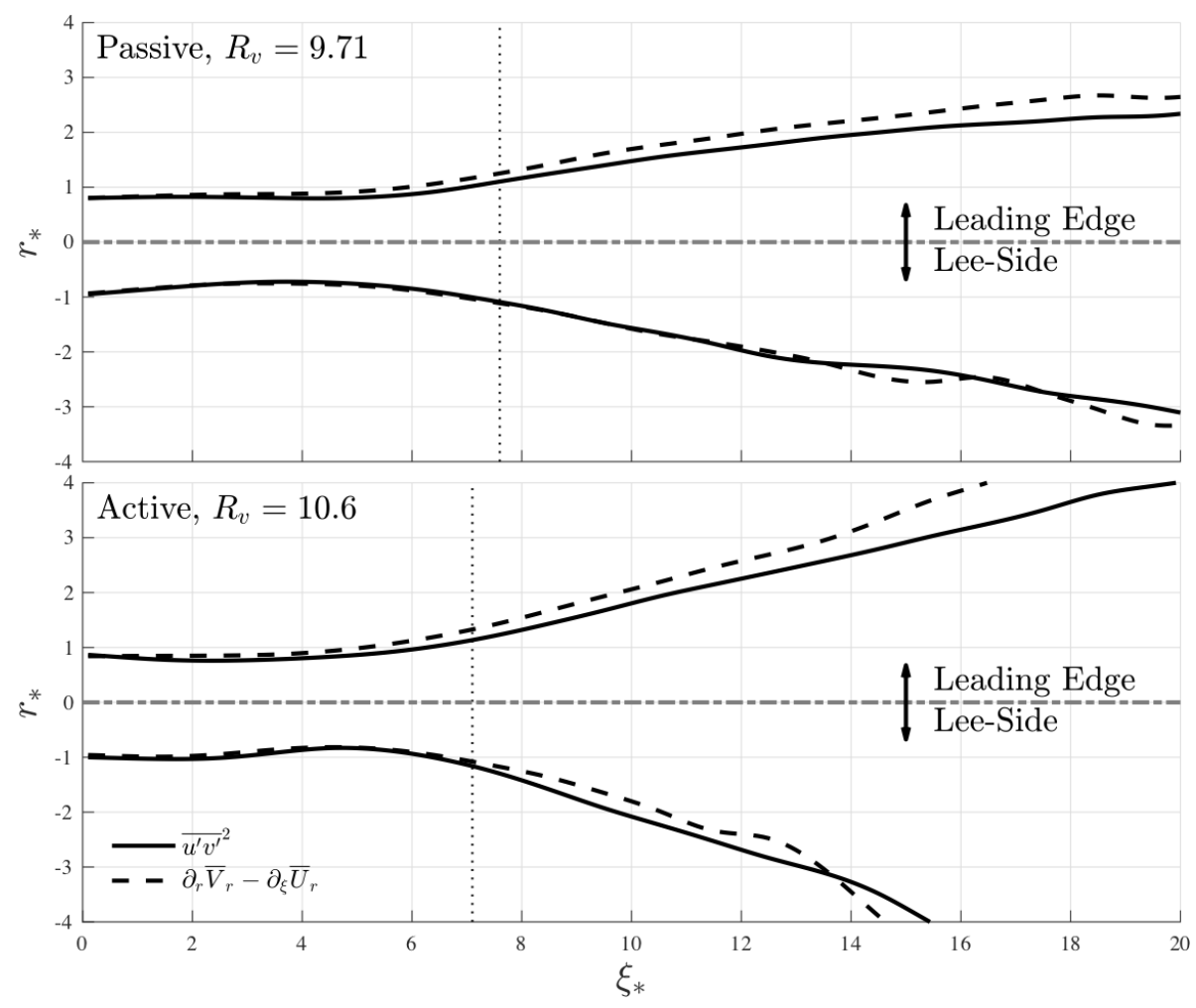

Figure 4.9: Comparison of the JCB definition from the squared shear stress profile $\left\langle u^{\prime} v^{\prime}\right\rangle_{r}^{2}$ and the squared vorticity $\left\langle\partial_{r} \bar{V}_{r}-\partial_{\xi} \bar{U}_{r}\right\rangle^{2}$. The shear stress profile allowed for definition farther downstream.

can be found in Appendix A. Within the near-field of the jet, the shear stress and vorticity both provided good agreement on the shear layer location. After the convergence of the shear layers, the definitions began to differ. On the leading edge, the vorticity described a wider jet than the shear stresses. On the lee-side, the same trend occurred with large variability for far-field identification, primarily due to the jet becoming indistinguishable in the mean flow statistics. Therefore, both definitions would be useful in the near-field, but the shear stress profile provided better far-field identification. Therefore, for all further results discussed in the subsequent sections of chapter 4 , the shear layer edges have been evaluated using 


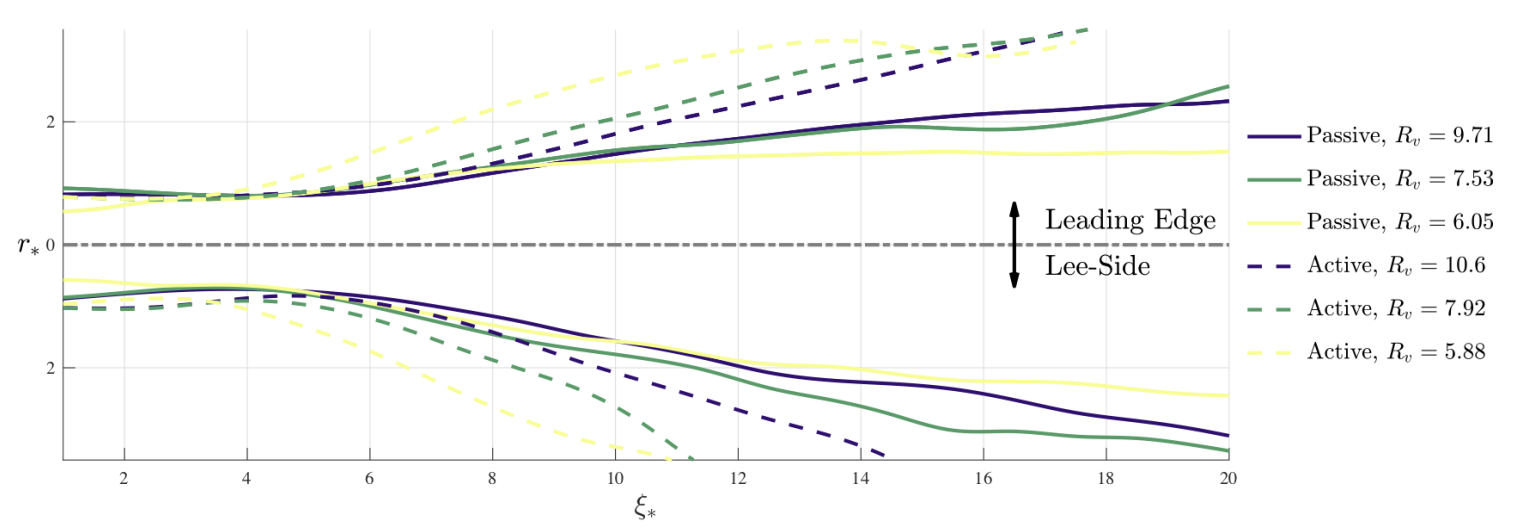

Figure 4.10: The jet cross-flow boundary at the outer edge of the shear layer for PG (solid) and AG (dashed). Three representative cases (with similar $R_{v}$ ) for $P G$ and $A G$ are shown.

the shear stress profile. In addition, the edges have been defined as the jet-to-crossflow boundary (or JCB).

Figure 4.10 displays the shape of the JCB for three similar $R_{v}$. Initially, the jet remains narrow $\left(r_{*}< \pm 1\right)$, with a similar half width on the leading edge and leeside. Then the jet narrows, reaching a minimum radius before expanding outwards after the convergence of the shear layers. As identified by Kelso et al. [59], this decreasing radius appears as the jet begins to bend downstream. On the leading edge, the shear layer is compressed due to the cross-flow applying a body force to the jet. On the lee-side, the formation of counter rotating vortices decreases the center-plane radius (similar to figure $9 \mathrm{~b}$ in Kelso et al. [59]).

Plotting the width of the jet, $\delta_{*}$, the growth before and after the collapse of the core were measured through the scaled length $\xi / \xi^{0}$ in Figure 4.11. For all cases, after the collapse of the core, both the leading edge and lee-side began to expand. For PG cases, the expansion remained similar for high $R_{v}$ while decreasing for low $R_{v}$ as the jet was close to the floor and could not expand more. This suggested that 


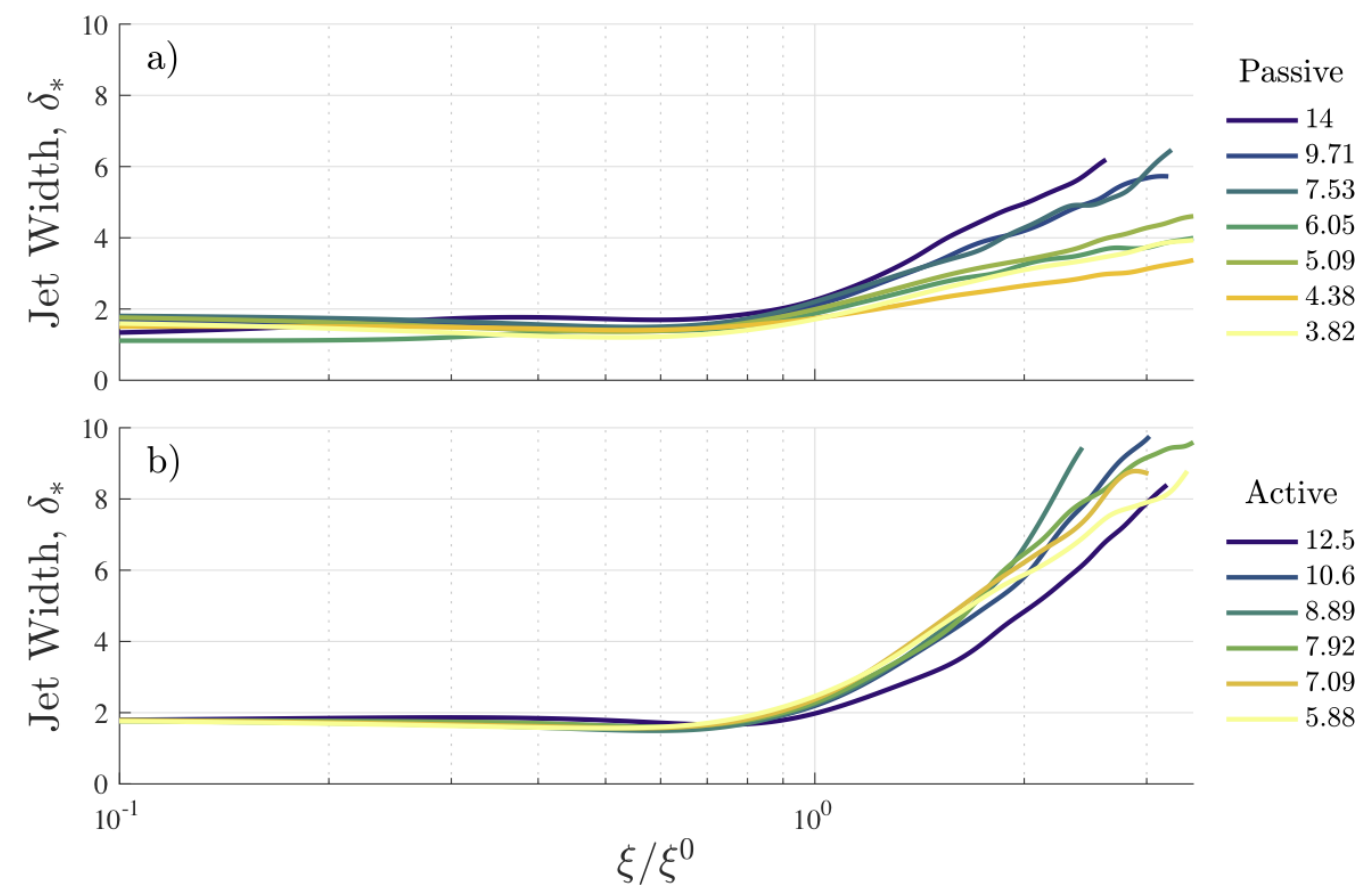

Figure 4.11: The width of the jet as a function of the scaled jet length, $\xi / \xi^{0}$ for all center-plane cases.

in a low turbulent environment, the entrainment that contributes to the expansion of the shear layer was reduced. For the AG cases, the opposite happens. As $R_{v}$ is decreased and the jet was reoriented downstream, the presence of turbulence in the cross-flow allowed greater expansion of the shear layer.

Within the near-field $\left(\xi / \xi^{0}<1\right)$, both the PG and AG cases were similar. The jet remained nearly a constant width of $\delta \sim 1.5-2$ approaching the collapse of the core. A minimal drop in width was observed near $\xi^{0}$, followed by a rapid expansion. For the PG cases, as $R_{v}$ decreased, the expansion of the jet and resulting width, $\delta_{*}$ decreased as well. This was a result of the body forces and floor preventing both leading edge and lee-side expansion. In comparison, the AG width collapsed after $\xi^{0}$ for low $R_{v}$. As observed in the inflow conditions, the length scale of the turbu- 
lence increased notably with inflow velocity. Therefore, with larger turbulent eddies present, the shear layer expanded into the cross-flow as the viscous contributions increased and overpowered the inertial body forces that reduced expansion in PG cases.

\subsubsection{Transport Analysis}

Full descriptions of the shape of the jet advance the understanding of JICF by identifying the increased mixing and expansion of the jet in high inflow turbulence. As shown earlier in Figure 4.1a and b, the extent of the lee-side recirculation region and wake region boundary layer were altered by high turbulence intensity cross-flow. Note that Figure 4.6 identified that the increase of turbulence kinetic energy that peaked at the collapse of the potential core highlighted a transfer from inertial to turbulent momentum and energy.

\subsection{4a Jet Transport}

To better understand the development of the jet and lee-side, changes to inertial and Reynolds stress transport of the Reynolds Averaged Navier-Stokes equation were investigated. The transport terms were first evaluated along the jet axis, $\left(r_{*}, \xi_{*}\right)$. A control surface extending the width of the shear layer was defined at each $\xi_{*}$ and equations (2.6), (2.7), (2.10), and (2.11) were evaluated using the mean flow statistics and Reynolds stresses. Figure 4.12 presents plots for two similar $R_{v}$, as a function of the scaled length $\xi / \xi^{0}$, to observe changes relative to the collapse of the potential core.

Similar to a turbulent free-jet, the majority of the inertial momentum and en- 

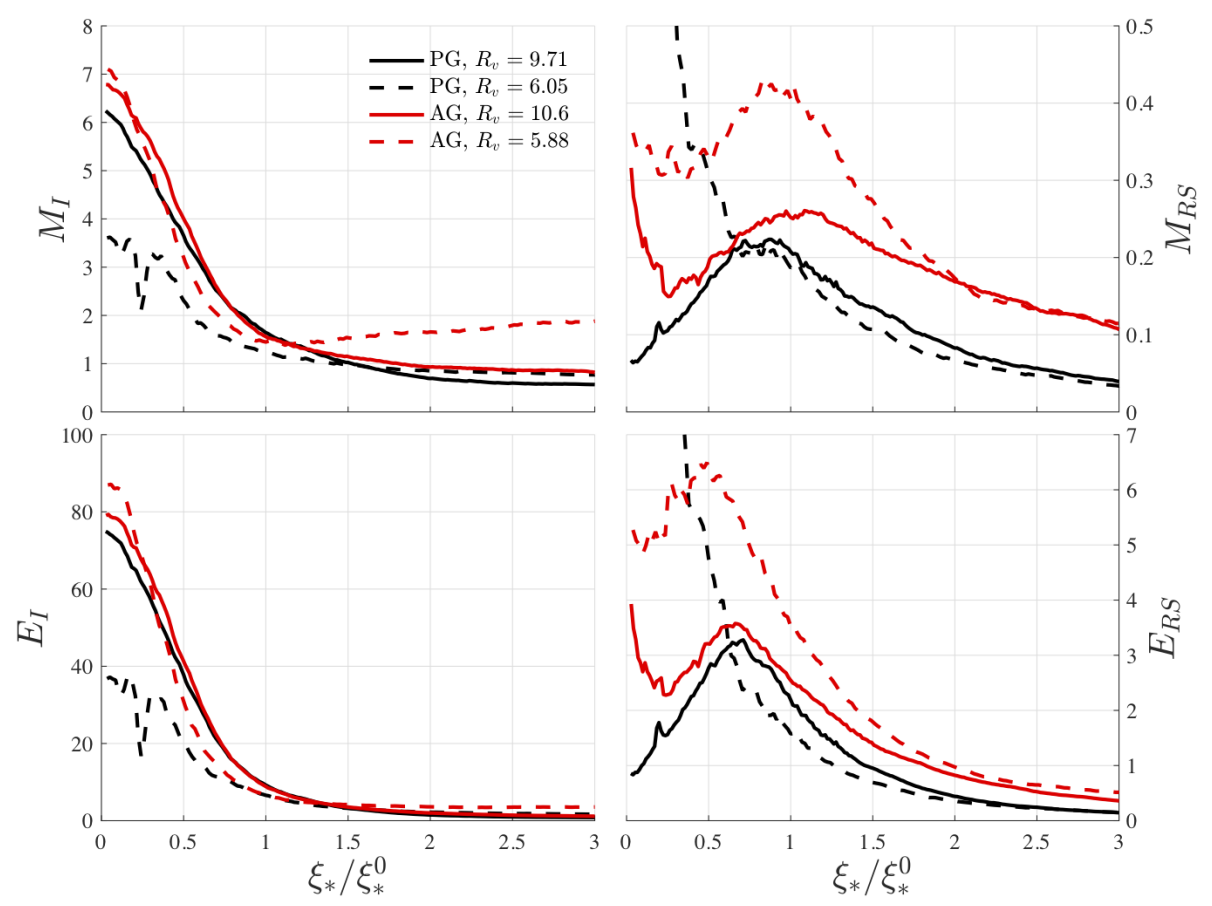

Figure 4.12: Integrated momentum and energy transport terms from Chapter 2.1 evaluated across the width of the jet for two similar $R_{v}$.

ergy at the exit was lost over the length of the jet. Losses across inflow conditions collapsed when scaled by the $\xi^{0}$. As the shear layers expand and vortex systems develop, a significant portion of the inertial momentum and energy would be lost. In the evaluation of the Reynolds stress contributions, the formation of the shear layer increased the presence of turbulence, but only reaches $\sim 5-10 \%$ the inertial losses. After the collapse of the core at $\xi^{0}$, the inertial terms began to converge and the Reynolds stress contributions reached a maximum, declining in magnitude and converge in the far-field. While the inertial terms behaved similarly, increases in Reynolds stress contributions were found in AG cases that persisted farther downstream. Increases of $50-100 \%$ from similar PG cases and changes across $R_{v}$ were observed despite negligible variations in the PG cases. This could be attributed to 

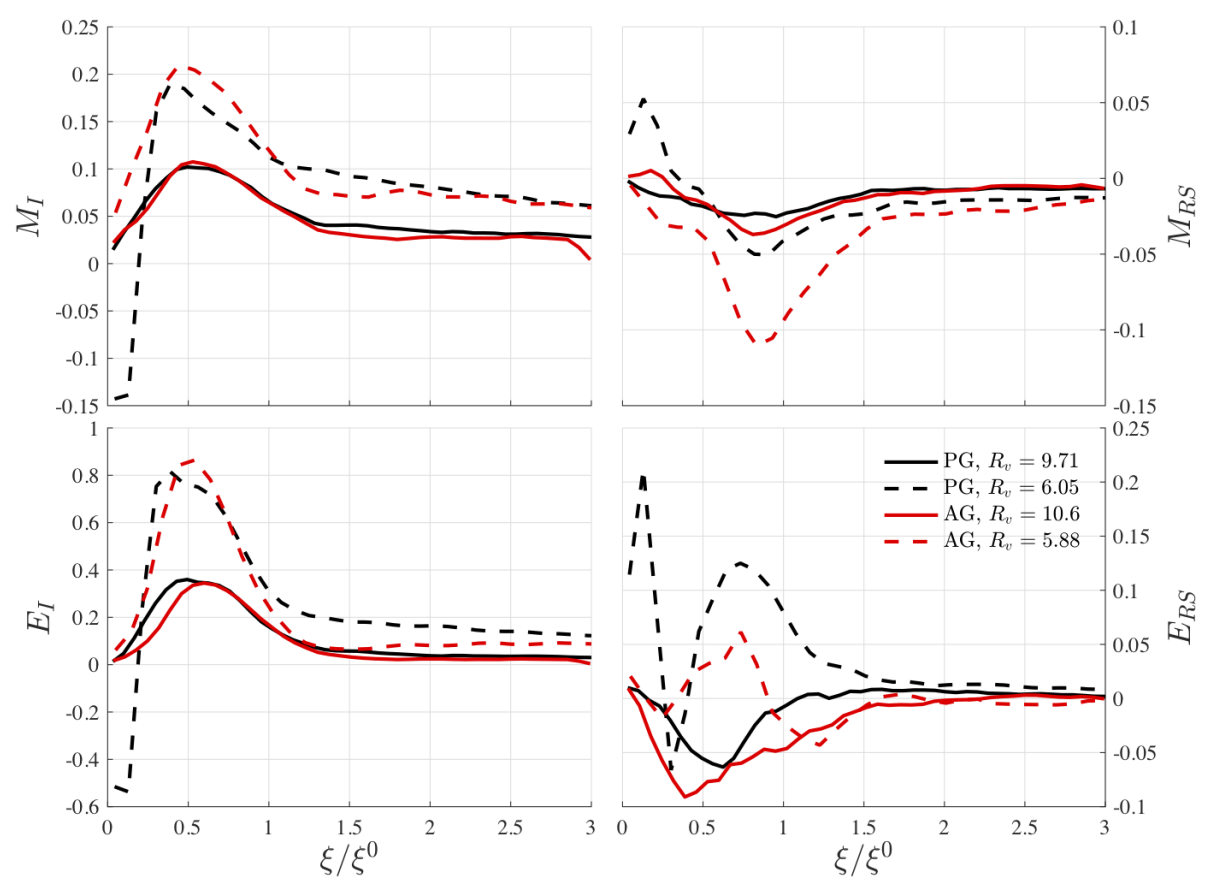

Figure 4.13: Integrated transport terms from Chapter 2.1 along the leading edge and lee-side JCB with the normal directed into the jet (positive for entrainment).

the changes to the shear layer expansion and entrainment (from Figure 4.11). In low inflow turbulence, the expansion was similar across cases. In high inflow turbulence, increased cross-flow velocity yielded greater expansion into the surrounding flow.

At the shear layer edge, entrainment is both a viscous and large scale phenomenon. Therefore, the changes to momentum and energy transport were additionally explored by measuring the transport through the JCB. The inertial and Reynolds stress terms were integrated from $\xi=\left[0, \xi^{0}\right]$ along the leading edge and leeside JCB to help focus on the decreases in inertial terms and increases in Reynolds stress contributions. The normal was defined into the jet to establish entrainment as positive. The results are presented in Figure 4.13.

The inertial terms identify entrainment that occurred within the near-field. 
While truly smaller than the exit profile of the jet (less than 1\%), the magnitude of entrainment increases with inflow velocity and could be attributed to engulfment at the edge of the shear layer. As the cross-flow velocity increased, more inertial momentum and energy was contained within the cross-flow. After the collapse of the core, the PG cases appeared to converge while the AG cases continued to decline further downstream. Therefore, the difference in the far-field was most likely due to the increased entrainment for AG cases as turbulent cross-flow was entrained by the turbulence jet.

The diffusion term, $M_{R S}$, confirms that the Reynolds stress contributions were lost through the JCB, with the largest losses immediately before $\xi^{0}$. With increased turbulence intensity, the magnitude of the losses doubled, an indication of increased expansion of the shear layer. The energy flux, $E_{R S}$, was similar for low cross-flow velocity. Energy moved out of the jet as viscous nibbling imparted the surrounding cross-flow with turbulence kinetic energy. As the cross-flow velocity increased, a region of entrained energy was present before $\xi^{0}$. For low inflow turbulence, the entrained energy persisted into the far-field. Near the collapse of the potential core, as the shear layers converge, the transport changed direction and an equal quantity of turbulent energy was expelled in the AG cases. This shift in transport, which coincided with the collapse of the core, was most likely the result of the rapid expansion of the shear layer. With the presence of turbulent fluctuations in the cross-flow, the jet was able to expand into the cross-flow through viscous nibbling [41, 42]. This was confirmed through quantification of the production of turbulence, $\mathcal{P}$, defined as $\mathcal{P}=\overline{u_{i}^{\prime} u_{j}^{\prime}}\left(\partial \bar{U}_{i} / \partial x_{j}\right)$, and presented in Figure 4.14.

Similar to the energy flux, the production peaked before the collapse of the core. 


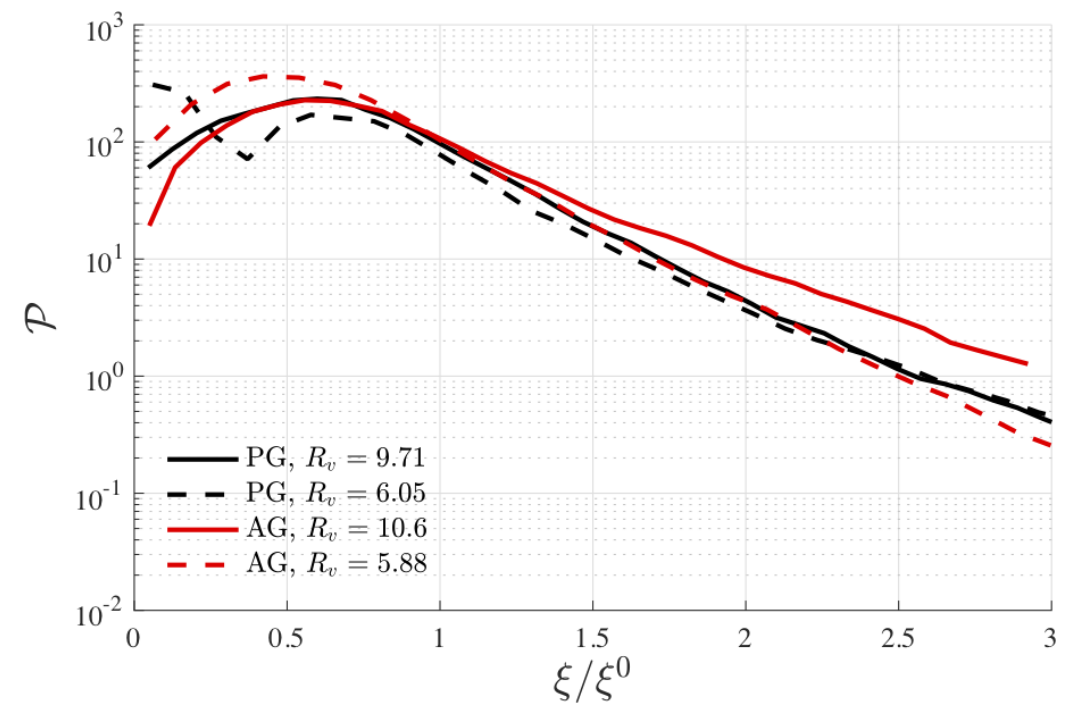

Figure 4.14: Turbulence energy production, $\mathcal{P}$, averaged across the width of the jet.

The greatest production of turbulence kinetic energy, which is near 50\% larger than the exit inertial energy, was present when the shear layers began to converge. The decrease immediately before the collapse of the core corresponded with the expansion of the shear layer into the inflow and wake region (from Figure 4.10). There were no observable changes to turbulence production in the presence of high turbulence intensity cross-flow. Therefore, the change in direction of energy flux was not a result of increased energy within the jet but instead the transport of energy out of the jet.

\subsection{4b Center-plane Wake Region}

To identify the lost Reynolds stress contributions observed in Figure 4.13, transport analysis was applied to measure the wake deficit. Due to the body forces on the leading edge, the majority of the transport through JCB before $\xi^{0}$ was assumed to 


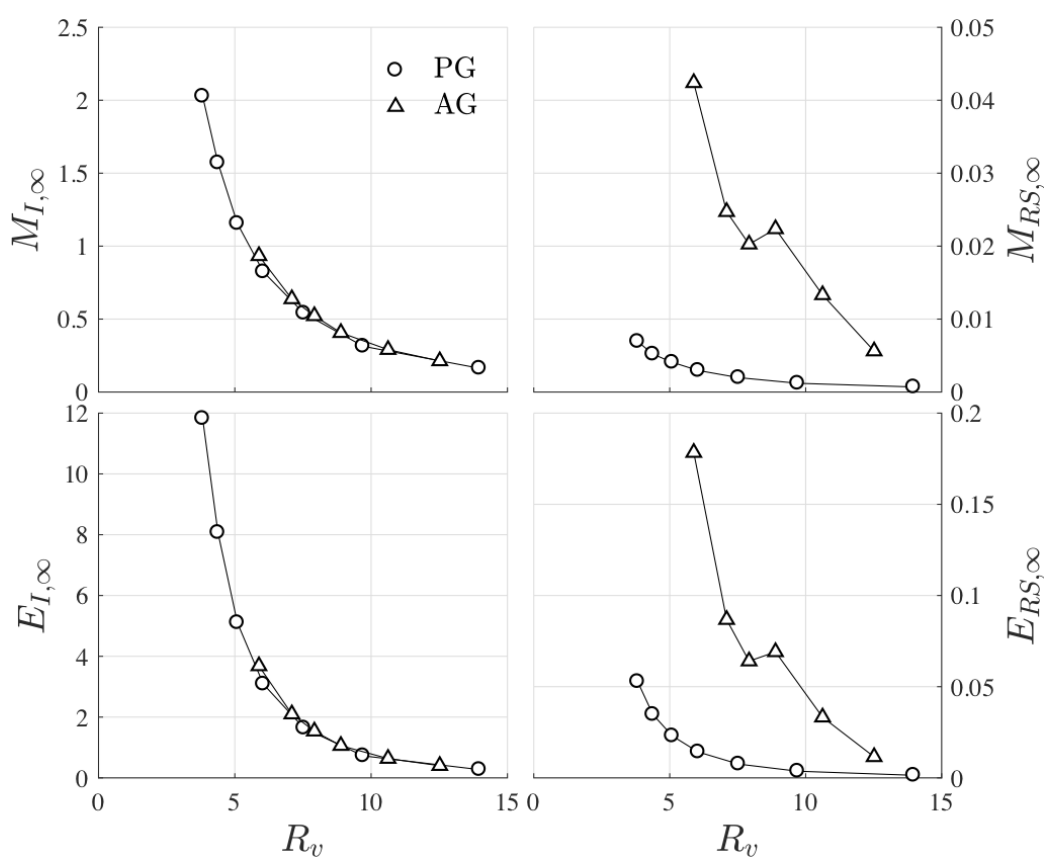

Figure 4.15: Momentum and energy transport of the inflow approaching the jet from $y_{*}=0 \rightarrow \xi^{0}$ for all cases.

be directed into the wake region. Therefore, the momentum and energy transport was compared upstream of the jet in the cross-flow, $M_{\infty}$ and $E_{\infty}$, and on the leeside moving downstream. The wake deficit was then computed as $\Delta M / M_{\infty}$ where $\Delta M=M_{\infty}-M\left(x_{*}\right)$. The control surfaces extended from $y_{*}=0 \rightarrow y^{0}$ to encompass the near-field region with the normal defined downstream.

To further describe the transport in the wake, the inflow was first quantified in Figure 4.15. The inertial terms were considered independent of inflow condition and collapsed as a function of $R_{v}$. The Reynolds stress contributions highlighted the increased turbulence intensity with a significant increase in diffusion and energy flux by nearly an order of magnitude.

The momentum and energy within the wake were then evaluated moving away from the jet in Figure 4.16. The downstream distance was defined as $\Delta x=x_{*}-x_{*}^{J C B}$, 

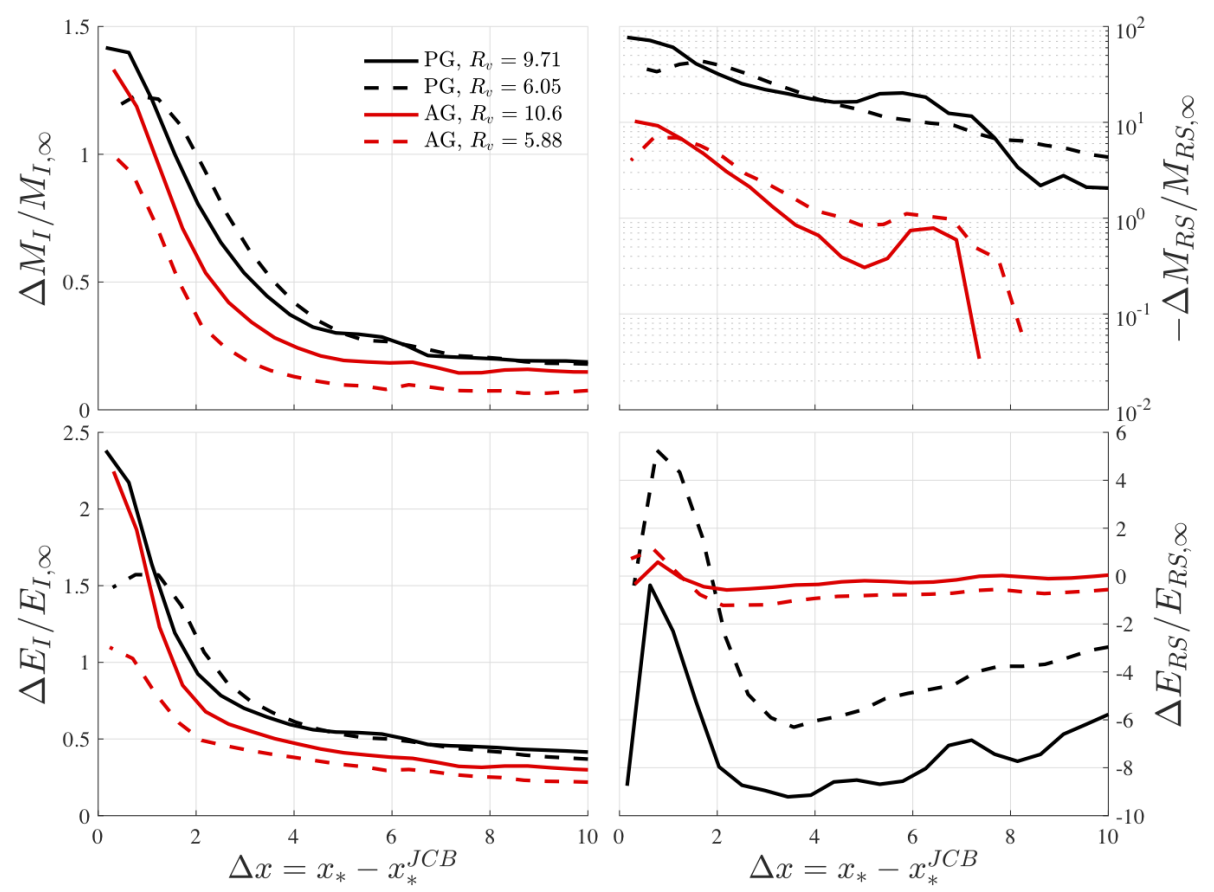

Figure 4.16: Momentum $\Delta M / M_{\infty}$ and energy $\Delta E / E_{\infty}$ transport deficit from upstream of the jet $\left(M_{\infty}\right)$ to lee-side wake region.

where $x_{*}^{J C B}$ was the downstream location of the lee-side JCB. The transport deficit was then described similar to bluff body wakes. The results yielded profiles of $M_{I}$ and $E_{I}$ that were similar to bluff body wake behavior. Both $M_{I}$ and $E_{I}$ were $>1$, due to the recirculation region that draws fluid toward the jet, while all remaining fluid continued downstream, decreasing the deficit as the wake recovers in the far field [30]. The same was observed for low inflow velocity, with additional inertial momentum and energy entrained in the lee-side recirculation region. The near wake contained approximately $50 \%$ of the inflow momentum and approximately $150 \%$ of the energy. The majority of the cross-flow would not be entrained on the lee-side, but the large magnitude present indicated the jet imparts inertial momentum and energy on the cross-flow. 
As the inflow velocity was increased, the magnitude of $M_{I}$ and $E_{I}$ decrease in the near-wake. The reduced size of the wake region due to increased bending of the jet decreased the magnitude of entrained inertial momentum and energy. For the PG cases, the near-wake changes did not influence the far-field wake. By $\Delta x_{*}=4$, the PG cases converged, allowing the far-field wake to be described independent of inflow conditions. With increased turbulence intensity, the recovery of the wake remains dependent on inflow velocity. The profiles appeared similar but did not converge downstream.

The Reynolds stress contributions described the wake recovery further. The diffusion, $M_{R S}$, was plotted as $-\Delta M_{R S} / M_{R S, \infty}$ to account for significant increase in the near-wake. For the PG cases, the wake was two orders of magnitude greater than the inflow. This region has been known to be highly turbulent due to the vortex shedding that had been observed previously [3]. These turbulent features persist far downstream and wake recovery was not observed in the window. Within the near wake, the AG deficits were nearly an order of magnitude smaller than the PG cases (the same as the inflow profiles). The wake recovery was also observed, as $M_{R S}$ decreases below the inflow conditions by $\Delta x=8$ downstream. The nearwake was highly turbulent but the presence of high cross-flow turbulence reduced the difference and allowed the fluctuations within the wake to spread off the centerplane. The energy flux behavior was similar downstream, with the PG cases nearly an order of magnitude greater than AG.

Closer to the jet, the near wake behavior was vastly different. For low inflow velocities, $E_{R S}$ was small and directed downstream. As the inflow velocity increased, the energy flux in the near-wake was directed upstream towards the jet. Most likely 
as the result of the counter-rotating vortices, the increased turbulence closer to the jet accounted for the entrained $E_{R S}$ observed in Figure 4.13. The shed wake vortices were entrained by the jet along the JCB, as observed by Fric \& Roshko [3]. In the present data set, as the high inflow velocity deflected the jet downstream closer to the floor, the spike in $E_{R S}$ was likely the results of increased vortex strength increasing the entrainment. This result was dramatically muted for the AG cases. Coupled with the increased wake recovery, it was posited that the presence of turbulence allowed the vortex systems on the lee-side to dissipate quickly, while allowing the surrounding flow to return close to inflow conditions.

\subsubsection{Summary}

Collection of instantaneous snapshots of the flow-field produced the velocity and Reynolds stresses which identified differences to the initial interaction as the incoming cross-flow turbulence intensity was increased. Most notably, the movement of cross-flow around the jet and entrainment within the recirculation region upstream of the unstable focus would be altered. As the cross-flow velocity increased, the jet bent closer to the floor, compressing the recirculation region of the wake. In the presence of increased turbulence intensity, out-of-plane movement and the boundary layer thickness downstream was reduced. From the Reynolds stresses, three turbu-

lent features were observed: the collapse of the potential core in $\overline{u^{\prime} u^{\prime}}$, the growth of shear layer vortices in $\overline{v^{\prime} v^{\prime}}$ and the expansion of the shear layer in $\overline{u^{\prime} v^{\prime}}$.

The centerline of the jet was defined using a power law relationship. A coordinate transformation reoriented the flow-field into components normal and tangential to the jet. The collapse of the potential core was observed when the radial normal 
stress peaked and was defined as $\xi^{0}$. The jet trajectory and peak location each showed a dependence on the scaling factor $R_{v}^{2} D$. The location of the JCB, defined as the location of negligible relative shear stress, $\overline{u^{\prime} v^{\prime}} r$, helped clarify the role of inflow turbulence on the rate of expansion. The presence of turbulence within the cross-flow allowed the jet to expand into the cross-flow more easily on the leading edge, leading to a far greater half-width. The lee-side showed less dependence on the turbulence intensity.

The measurement of transport through the jet highlighted the key features and standout observations from the statistics. The majority of inertial momentum and energy losses occurred before $\xi^{0}$. The Reynolds stress contributions were small but decreased after the collapse of the core. Measuring the transport through the shear layer identified small increases in inertial transport (entrainment) and turbulent transport leaving the jet. The increases and drops correlate well with the production of turbulence. Therefore, the jet was found to entrain inertial momentum and energy from the cross-flow, convert it to turbulence kinetic energy, which then entrained additional cross-flow through viscous nibbling.

For high inflow velocities, a spike in energy flux into the jet was observed near the collapse of the core. Measurements within the wake observed upstream transport in the near-wake region. These were determined to be the highly turbulent wake vortices reattaching to the lee-side of the jet. High inflow turbulence significantly reduces this behavior while increasing wake recovery.

In total, highly turbulent inflow was found to a) increase shear layer expansion due to free-stream fluctuations, b) increase wake recovery due to mixing with crossflow outside the wake, and c) change the near-wake recirculation region. Therefore, 
an investigation of the changes in turbulent features would be important in any simplified description of jets in cross-flow.

\subsection{Jet Slices $(x-z)$}

To investigate the variations in wake behavior, six PIV planes (outlined in Chapter 3.3) captured the near-field interaction and wake region before the collapse of the potential core. From Table 3.3, three inflow velocities were tested that provided a range of Reynolds numbers $\left(\operatorname{Re}_{\infty}\right)$ that will create varying wake behavior. Re was computed at multiple heights as $\operatorname{Re}_{\infty}=\bar{U} D / \nu$, using the upstream mean velocity, $\bar{U}$, to describe the behavior of the interaction similar to bluff body wakes.

In Figure 4.17 , the streamwise $\left(\bar{U}_{*}\right)$, vertical $\left(\bar{V}_{*}\right)$, and out-of plane velocies $\left(\bar{W}_{*}\right)$, were presented at $y_{*}=4$. Three distinct features were present within the flow-

field: the reduction in streamwise velocity, $\bar{U}_{*}$, approaching the leading edge; the accelerated flow around the sides of the jet; and the wake region where a momentum deficit existed.

As flow approached the jet, the deceleration in $\bar{U}_{*}$ highlights similarities to bluff body dynamics. The deceleration of velocity at the leading edge was due to a stagnation point that prevented the cross-flow from penetrating the jet. Off the centerline $(z \neq 0) \bar{W}_{*}$ would be equal and opposite as flow was redirected around the jet. The cross-flow was accelerated around the jet (the highest magnitude $\bar{U}_{*}$ ) and entrained on the lee-side. Similar to the results observed in Figure 4.1, a recirculation region existed where $\bar{U}_{*}<0$ and $\bar{V}_{*}>0$ as cross-flow was trapped within this region, imparted with vertical momentum and entrained by the jet in a secondary flow along the lee-side JCB. Moving away from the jet, the $\bar{U}_{*}$ crossed 


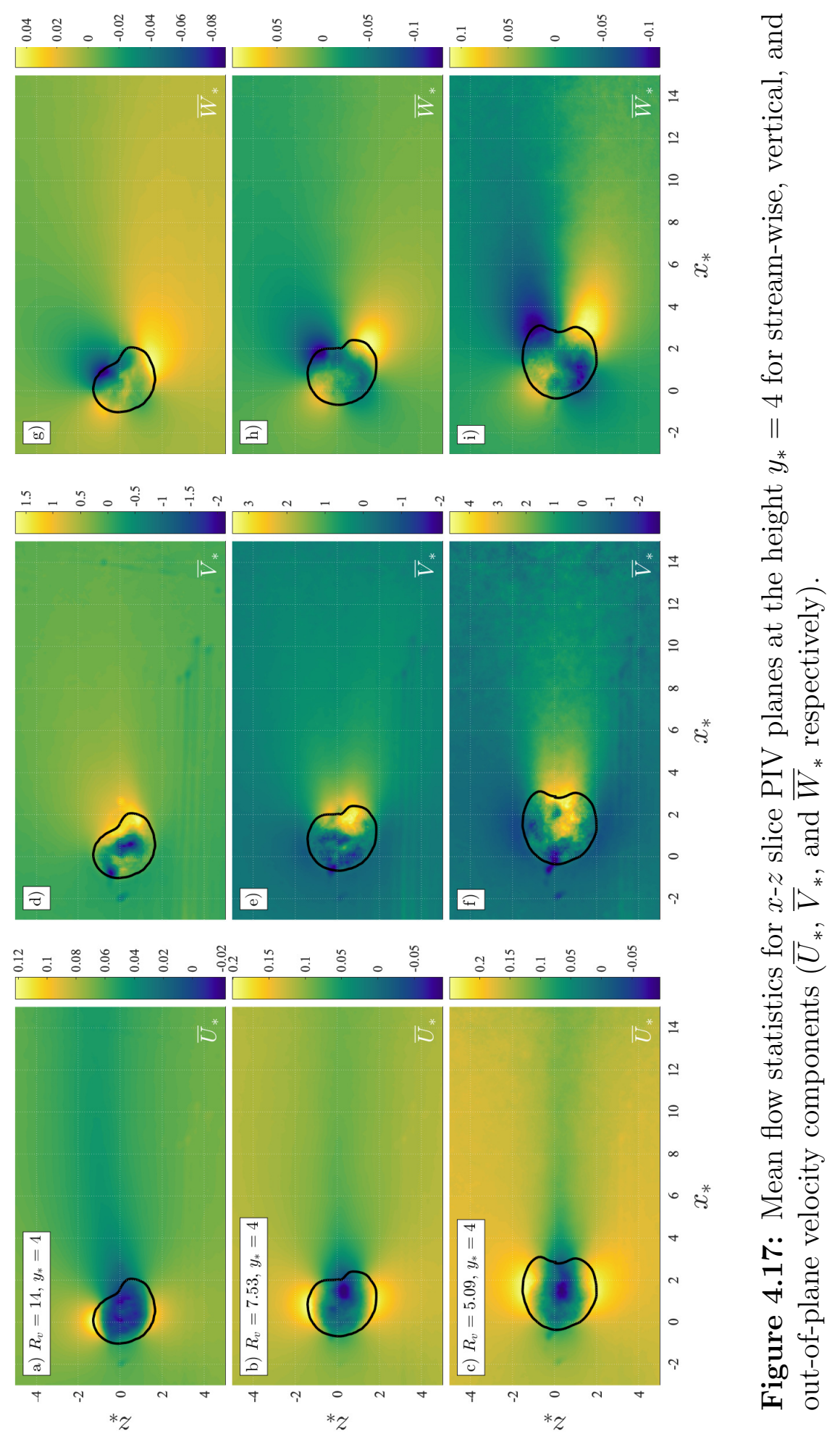


zero and a wake extended to the edge of the window. For bluff bodies, the wake was a product of the drag forces imparted on the body. When Re becomes sufficiently large, vortices were formed within the recirculation region and shed downstream. For a jet in cross-flow, it was assumed that this behavior would change for two reasons. First, the jet is not a rigid body and oscillations will likely form at low Re. Second, the jet will bend downstream, which in turn will entrain the shed vortices [3].

For the low inflow velocity case in Figure 4.17a-c, the wake drifted off the centerline but re-converged behind the jet for increasing cross-flow velocity. This was a product of the complex shape of the jet. In classical bluff body wake investigations, the drift off the center-line did not appear due to the rigid shape and symmetric area. The jet is a fluid body that deformed due to body forces and turbulence, therefore any instability favored on one side of the jet would cause a loss of symmetry. As the inflow velocity was increased, the same instabilities were present, but the increased momentum of the cross-flow prevented significant deformation, yielding a more symmetric wake.

This assumption was confirmed by evaluating the JCB at each height. The ratio of unresolved snapshots to total snapshots at each location, $N_{0}\left(x_{*}, z_{*}\right)=n_{0} / n_{s}$, was recorded from the over-seeded snapshots (Figure 4.18a). The origin of the jet was defined by the downstream centerline location from the centerline data (Figure 4.3). A line was extended to the edge of the window and $N_{0}$ was interpolated. The resulting profile was fit with a Gaussian exponential function to define the width at half maximum. This protocol was chosen to account for the dynamic boundary of the jet. This was repeated by revolving the line around the center of the jet to 

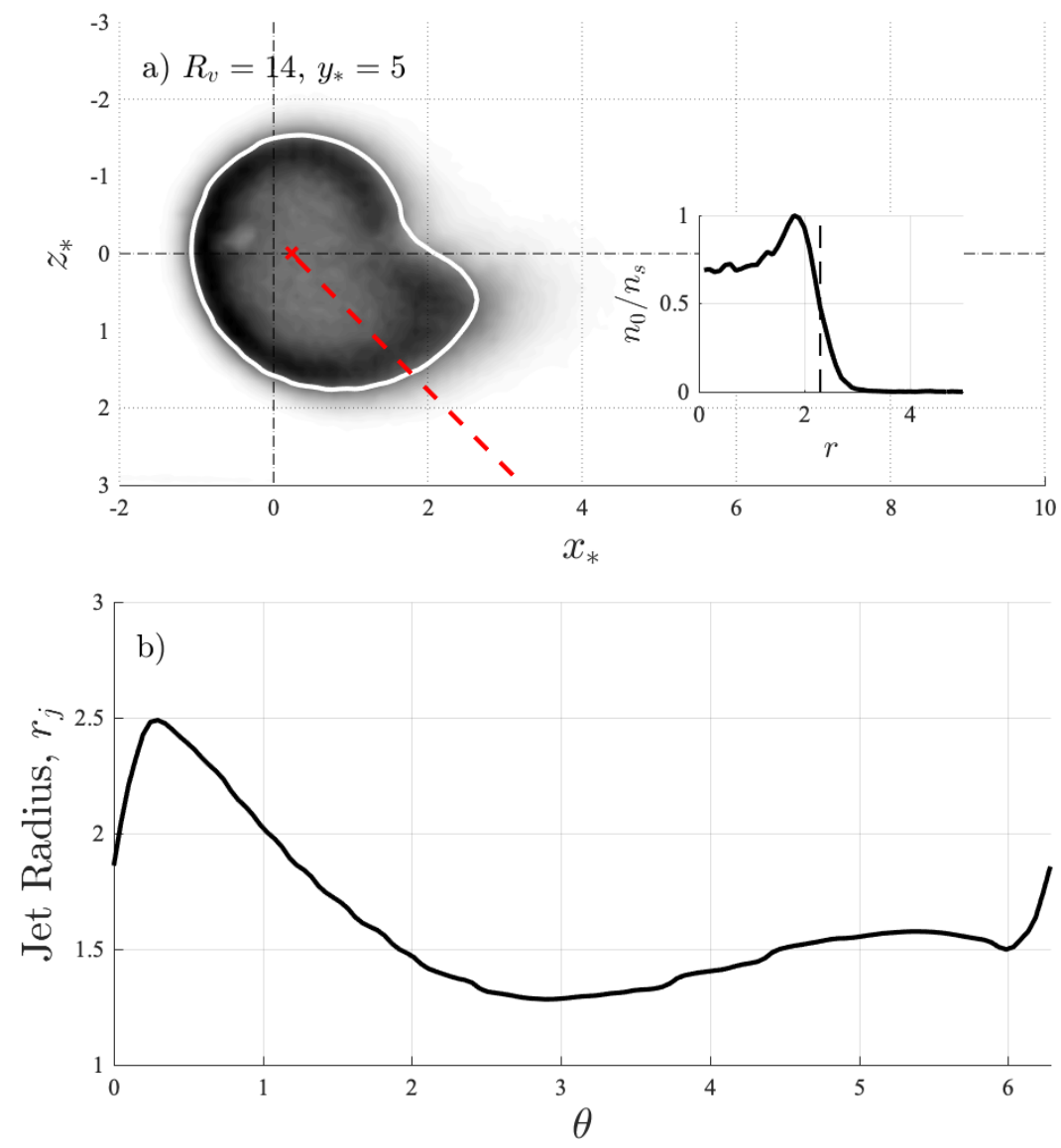

Figure 4.18: Method of defining the edges of the jet in the $x-z$ plane for $R_{v}=14, y_{*}=5$. For each case (panel a), a series of lines were extended from the center of the jet, allowing a fraction of unresolved data points to be recorded. The edge of the jet was defined as the width at half maximum of a Gaussian fit. This was repeated for b) $\theta=0 \rightarrow 2 \pi$ to provide the radius of the jet, $r_{j}(\theta)$.

define the radius of the jet as a function of the angle, $r_{j}(\theta)$.

A three dimensional volume was constructed from the conic sections in Figure 4.19. As expected, all self-similarity was absent at $y_{*}=1$ within the jet [48] and a highly skewed profile remained. For the low $\operatorname{Re}_{\infty}$ case (Figure 4.19a), the skewed profile expanded through $y_{*}=5$ before it began to recede and a more symmetric profile formed. For the high $\operatorname{Re}_{\infty}$ case (Figure 4.19b), the skewed profile was present 

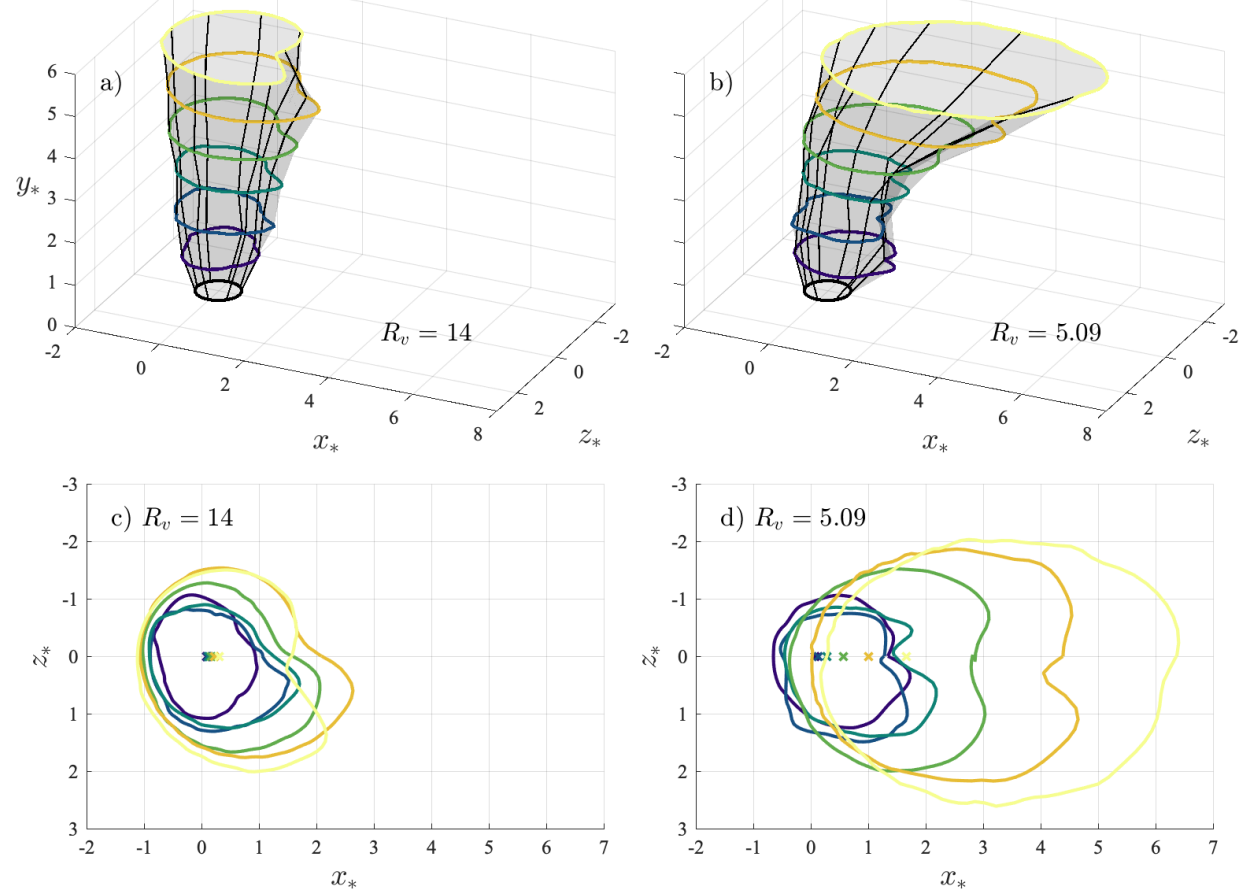

Figure 4.19: Three-dimensional jet volume construction (a, b) using the method in Figure 4.18 on each $x-z$ plane $(\mathrm{c}, \mathrm{d})$ for a low and high crossflow velocity case.

up to $y_{*}=3$ when the high inflow velocity provided uniform forcing to create a symmetric profile. The formation of two counter-rotating vortices was observed, but the skewed profile confirmed entrainment was not axisymmetric near the exit.

Similar to the JCB measurements on the center-plane (Figure 4.10), the leading edge did not expand into the cross-flow. The lee-side boundary expanded downstream, first through the shear layer vortices wrapping around the jet and forming two small peaks, as identified in Kelso et al. [59] (see Figure 9b in Kelso et al. for a full schematic). These peaks evolve into the counter-rotating vortices as they converged on the center-plane. The high out-of-plane component observed in the $x-y$ 

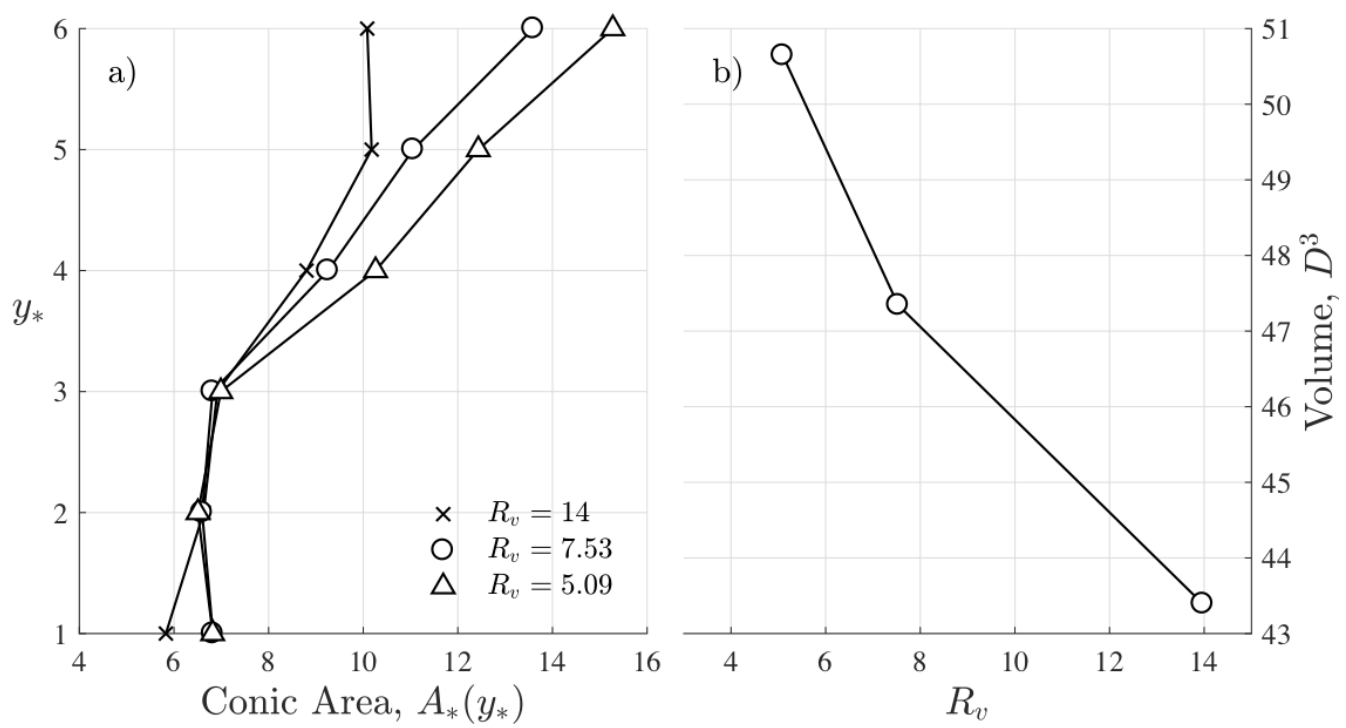

Figure 4.20: Area of the jet conic sections at each height and the integrated volume through $y_{*}=6$.

plane (Figure 4.1) coincided with these features, extending downstream and drawing cross-flow into the wake region. After the collapse of the potential core, occurring in $R_{v}=5.09$ between $y_{*}=5$ and 6 , the leading edge and lee-side boundaries began to expand. This was the same outcome observed in the previous section.

Computing the cross-sectional area, $A_{*}$ (Figure 4.20), a near constant area was observed in the near-field for $y_{*} \leq 3$. Despite changes in the conic profile of the jet, the formation of counter-rotating vortices extended the jet downstream out-ofplane, as the center-plane width (at $z_{*}=0$ ) was compressed on the lee-side. For $y_{*} \leq 3$, the jet did not expand but was deformed by the vortex systems. Once the vortex systems began to develop and the shear layer forms, the area increased. 

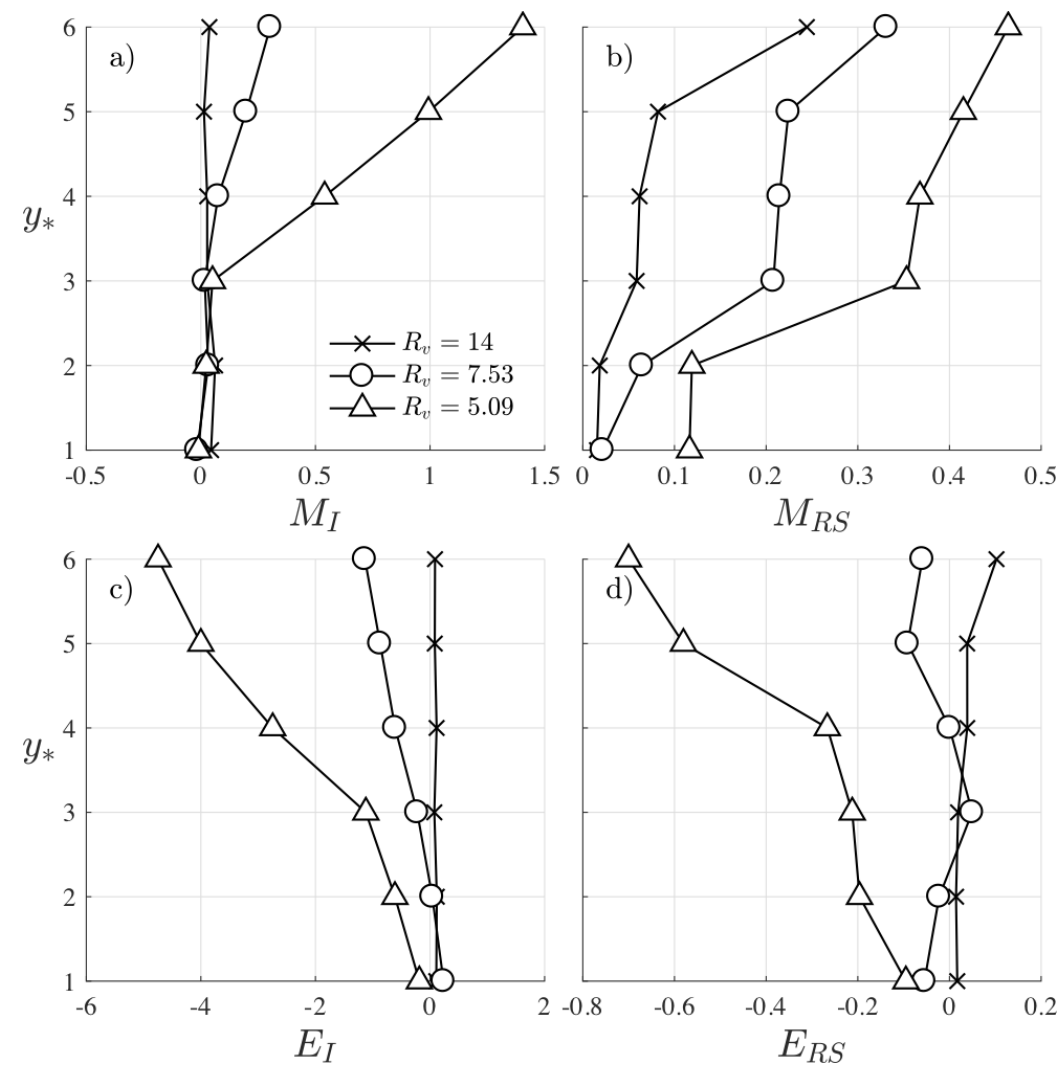

Figure 4.21: Net momentum and energy transport integrated along the closed area defined by the JCB. The positive normal was defined into the jet to denote entrainment with positive values.

\subsubsection{Transport Analysis}

To examine the mechanics reducing the jet expansion near the exit, the transport terms from 2.1 were evaluated. Along the defined JCB from Figure 4.18, the velocity components were transformed through the relative axis method (Appendix B) to provide the velocity components normal and tangential to the boundary. The normal was defined into the jet and the transport terms were integrated over the closed area. A positive value corresponded to transport into the jet.

In Figure 4.21a, the convection, $M_{I}$, was negligible through $y_{*}=3$, a confirma- 
tion that entrainment did not occur. In the $x-y$ plane (Figure 4.13 ), the entrained momentum increased until $\xi / \xi^{0}=0.5$ and then decreased, converging at a constant net transport. Including only the leading edge and lee-side, where cross-flow was directed into the jet and the width as compressed, the net transport was positive. With the addition of radial measurements around the entire jet, the deformation due to the vortex systems reduced the net convection to zero.

The diffusion term, $M_{R S}$, was positive as Reynolds stress contributions were entrained by the jet. As the counter-rotating vortices formed, the turbulent shear layer vortices were pushed around the radius of the jet and entrained on the lee-side. These large vortices, which contained high turbulent kinetic energy, were trapped on the lee-side and entrained into the jet.

For the energy terms, the role of inflow velocity was critical. Both the inertial energy, $E_{I}$, and energy flux, $E_{R S}$, described the turbulent features within the jet. Most importantly, this included the shear layer expansion and vortex systems. With increasing inflow velocity, the inertial energy expanding the jet increased. Close to the exit, where the jet did not expand, the shear layer formation and entrainment of deflected cross-flow to the lee-side of the jet was observed. The energy flux, a description of viscous nibbling at the JCB, did not begin to substantially grow until after $y_{*}>4$. The small magnitude features were most likely the formation of the shear layer and vortex systems that interact with the cross-flow.

This transport expanded the understanding of the near-field behavior. First, the presence of turbulence kinetic energy and negligible inertial transport near the exit indicate delayed entrainment. Second, the formation of the shear layer and vortex systems deformed the jet on the lee-side wake while the cross-flow prevented 


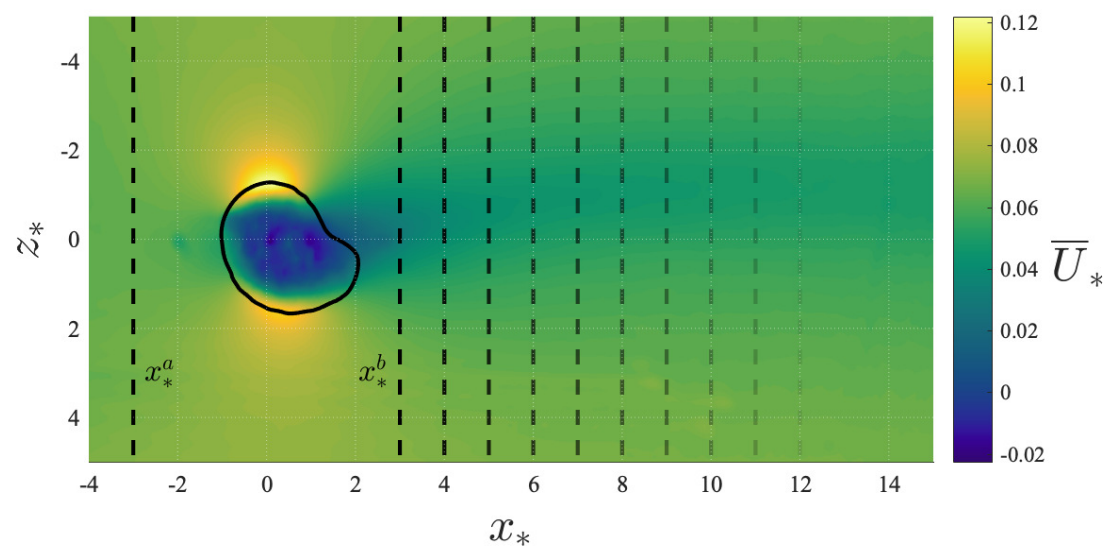

Figure 4.22: The upstream location, $x_{*}^{a}$, and downstream location, $x_{*}^{b}$, used to evaluate the momentum and transport deficit within the wake.

expansion elsewhere.

The lost energy was investigated through transport analysis within the wake. The momentum and energy deficit were determined under the same method as section 4.1.4b. Two locations were defined, the first upstream of the jet at $x_{*}^{a}=-3 D$ and the second at a location $x_{*}^{b}$ downstream of the jet (see Figure 4.22). The velocity profiles, $\bar{U}_{*}^{a}\left(z_{*}\right)$ and $\bar{U}_{*}^{b}\left(z_{*}\right)$, were used to compute the momentum deficit (Figure 4.23),

$$
\Delta U / U=\left(\bar{U}_{*}^{a}-\min \left(\bar{U}_{*}^{b}\right)\right) / \bar{U}_{*}^{a} .
$$

Due to the bending motion of the jet, it was plotted as a function of $x_{*}^{b}-x_{*}^{J C B}$, the distance from the lee-side JCB. For $\operatorname{Re}_{\infty} \sim 900$, the $\Delta U / U$ is greatest near the floor. Moving away from the floor $\left(+y_{*}\right)$, the deficit drops from $\sim 0.3$ to $\sim 0.15$ at $y_{*}=6$ as the jet entrained more momentum from the cross-flow as the shear layer grew. For increasing $\mathrm{Re}_{\infty}$, the momentum deficit near the floor increased. As $R_{v}$ was decreased, the deficit increased, dropping below $\Delta U / U=0.1$ by $10 D$ for 


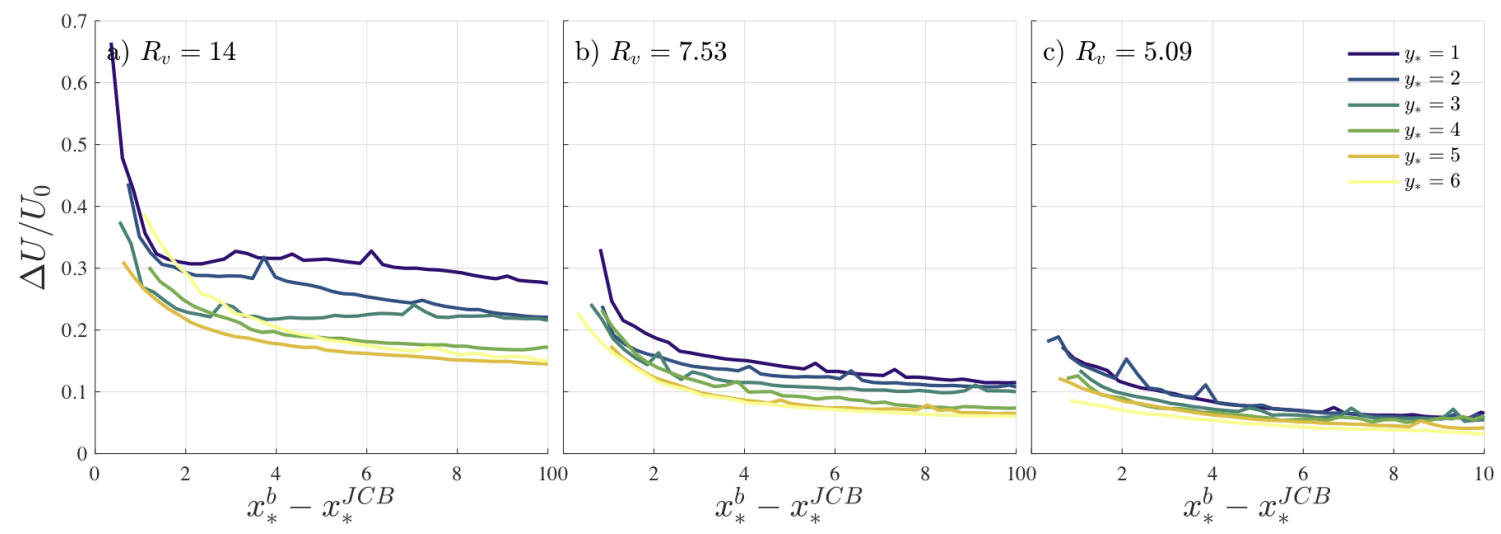

Figure 4.23: The wake momentum deficit, $\Delta U / U$, for each $x-z$ plane plotted as a function of $x-x_{J C B}$.

$\operatorname{Re}_{\infty} \sim 1600$ and by $3 D$ for $\operatorname{Re}_{\infty}>2000$. Therefore, more momentum was entrained by the jet as $R_{v}$ decreased.

Evaluating the momentum and energy transport terms through the wake allowed a different perspective for the results shown in Figure 4.16. Evaluated on the $x-z$ plane, the movement of fluid around the jet was accounted for and the disappearance of Reynolds stress contributions were investigated in Figure 4.24.

On the $x-y$ plane, the net inertial momentum within the wake decreased with distance due to the entrained $M_{I}$ present in the recirculation region. On the $x$ $z$ plane, the wake transport was nearly equivalent to the inflow at the JCB for low cross-flow velocity. Further downstream, the inertial momentum appeared to move vertically within the wake as it developed downstream. While momentum recovery was expected, $\Delta M_{I} / M_{\infty}$ increased moving downstream. With increasing height, the downstream magnitude decreased as the wake recovered to upstream conditions. With increased inflow velocity, the same trends were observed, but with an increase in $M_{I}$ of $\sim 10 \%$. From Figure 4.21a, the momentum deficit should 

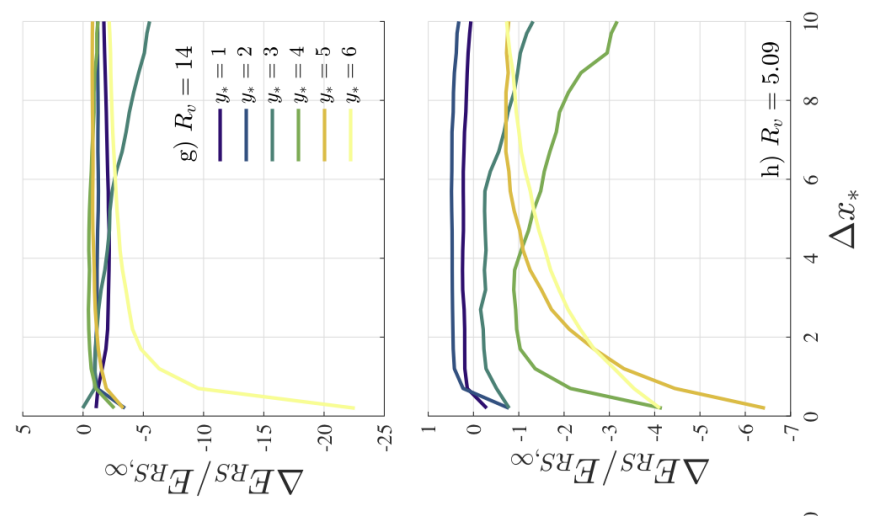

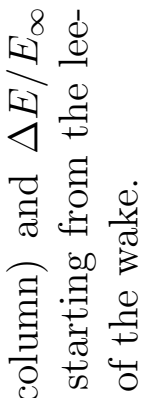
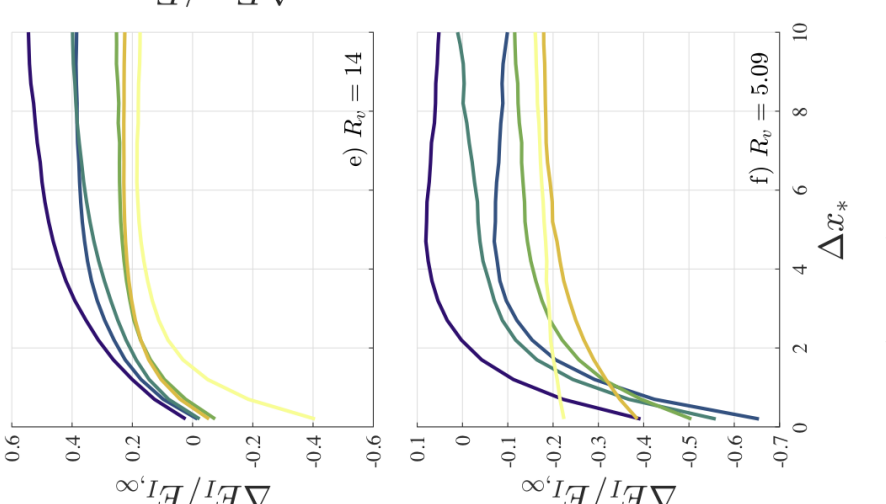

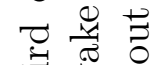

$3 \overrightarrow{7}$

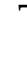

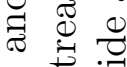

药

응

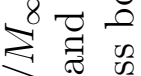

₹

$\exists . \exists$ ठ

.
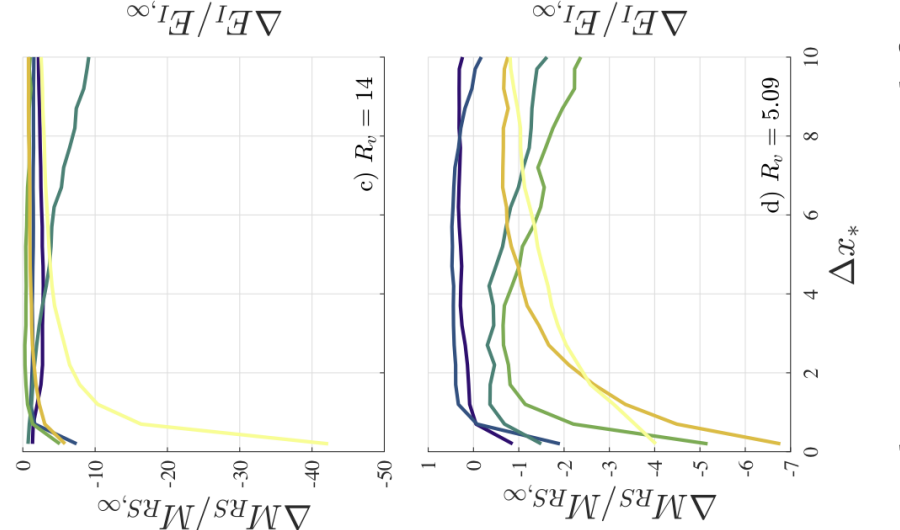

- 0

는

ㄴ.

की

元

¿ II

के

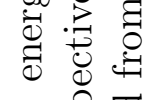

च.

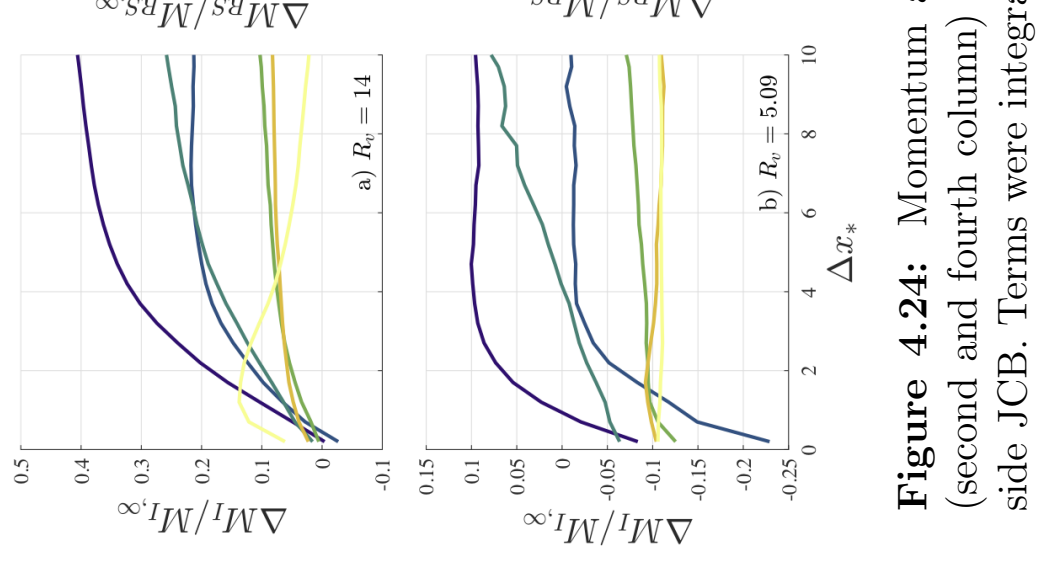


have decreased with height as more inertial momentum was entrained by the jet. Instead, for $y_{*}>3$, the transport appeared to remain constant throughout the wake at a greater magnitude than the inflow. This vertical movement was a result of the wake vortices. From Fric \& Roshko [3], the vortices "erupt" from the floor and reattach to the jet, drawing fluid from the boundary layer to the lee-side of the jet. Additionally, the presence of a secondary flow along the lee-side JCB draws entrained fluid from the recirculation region up along the lee-side JCB and into the counter-rotating vortices. This was present in the $\bar{V}_{*}$ component of Figure 4.17d-f. Therefore, the losses of inertial momentum were the result of the vortex systems entraining cross-flow near the jet that dissipated downstream.

The inertial energy, $E_{I}$, tells a similar story. In low cross-flow velocity, $E_{I}$ was nearly equivalent to the inflow on the lee-side and decreased in magnitude moving downstream. Close to the floor, the losses persisted throughout the wake, but for higher elevations, the inertial energy converged to a constant value at $\Delta x_{*}>5$. For increased cross-flow velocity, the inertial energy within the wake increased and was greater in magnitude than the inflow. In Figure $4.21 \mathrm{c}$, the strong increase in $E_{I}$ was a direct result of interaction with the jet. At high elevations, both the energy imparted on the cross-flow and the vertical movement of energy was observed in the vortex systems. While convergence was achieved similar to the low inflow cases, the wake contained up to $20 \%$ more inertial energy downstream. This was the same result observed in the $x-y$ plane.

The Reynolds stress contributions confirmed the presence of highly turbulent features in the near wake. The shed vortices, secondary flow and deformation of the jet increased the diffusion, $M_{R S}$, observed near the jet. Moving away from the 
jet, these features converged to a greater magnitude than the inflow. Interestingly, for elevations with the range of $2<y_{*}<4$, a continuous increase in diffusion was recorded. This region coincided with the top of the boundary layer within the wake (Figure 4.1a and b). Within the wake, the shed vortices were connected to the jet as they were shed downstream. After the jet was fully developed in the far-field, the trajectory was directed downstream. The counter-rotating vortices were still present and entrained cross-flow into the wake region. Therefore, this region of continued increase in Reynolds stress contributions was the interaction of momentum and energy entrained by the vortex systems and the boundary layer in the far-wake of the jet.

\subsubsection{Summary}

The introduction of the $x-z$ plane provided a different perspective on the behavior of the cross-flow interaction with the jet. The movement of cross-flow around the jet confirms bluff body behavior and the recirculation region and secondary flow are observed on the lee-side. A wake drift and skewed shape were observed in low cross-flow, and was corrected with increased cross-flow velocity.

Evaluation of the edge of the jet allowed direct calculation of the changes in both area and volume. The jet was found to remain a constant area close to the floor before expanding primarily downstream into the wake. Deformations on the lee-side boundary were the result of movement of shear layer vortices around the jet, which form the counter-rotating vortices. These were also observed for high cross-flow velocities.

Measurement of the momentum and energy transport through the jet identified 
nearly negligible entrainment close to the exit as the shear layers began to form. Additionally, the net momentum transport was entrained into the jet while the net energy transport was lost. This describes the contributions of the vortex systems in the entrainment of momentum from the cross-flow while the turbulent shear layer and high inertial energy of the jet expanded outward.

Evaluation of the wake region identified a few key observations. The presence of shed wake vortices and the secondary flow pulled momentum up from the floor. The movement of momentum decreased the inertial transport within the wake, preventing full recovery and the Reynolds stress contributions confirmed the presence of turbulent features in the near-wake that dissipate in the far-field. Within the elevation $2<y_{*}<4$, the highly turbulent features observed were the interaction of the entrained cross-flow from the counter-rotating vortices and the boundary layer. These observations confirmed the vortex systems and near-wake behavior are necessary in any description of entrainment.

\subsection{Proper Orthogonal Decomposition}

While turbulence was found to play a role in the expansion of the jet, a description of the energy content would allow identification of how features within the jet and wake region were influenced, as well. Utilizing snapshot proper orthogonal decomposition as described in Chapter 2.6, the fluctuating fields were decomposed to isolate the most energetic regions of the flow.

A total of 2500 and 3000 flow-fields were generated for PG and AG respectively. The fluctuating components were projected onto the POD basis to generate modes with eigenvalues proportional to the turbulence kinetic energy. Ordered from the 


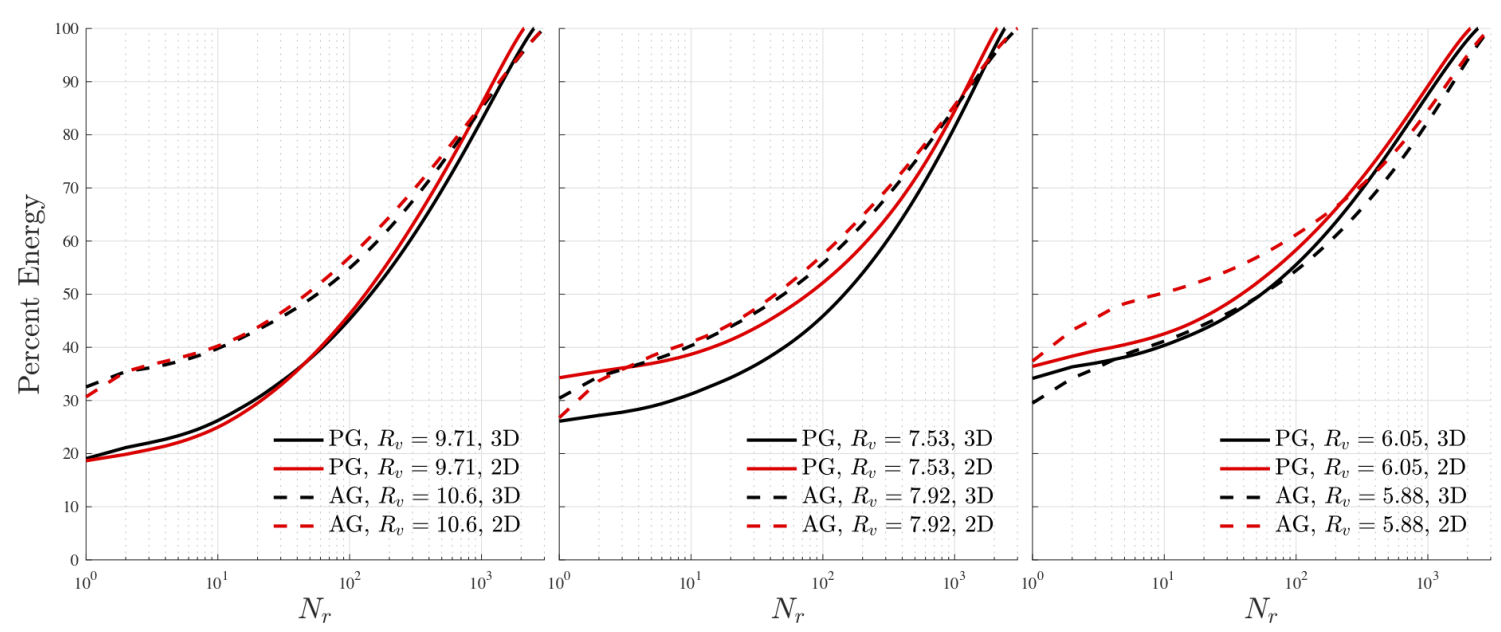

Figure 4.25: Cumulative (percent) energy per mode for each inflow condition as a result of POD on the relative fluctuating flow-fields.

most to least significant contributions to the total energy, all three velocity components were included to determine the importance of out-of-plane motions.

\subsubsection{POD Modes}

Each mode contains eigenvalues, $\lambda_{n}$, whose cumulative sum accounts for the total integrated turbulence kinetic energy. Represented as a percentage of the total energy, the cumulative energy is presented in Figure 4.25 for both two and threecomponents. For the PG cases, greater inflow velocity increased the cumulative energy contained in the first mode. Therefore the energy contained in the most significant features increased. Comparisons between two- and three-component decomposition identified that the out-of-plane component was not the most significant contributor. The greater increase in first mode cumulative energy for two-component and the gradual increase for three-component indicated this component is instead summarized in higher order modes. 
For the AG cases, the two- and three-component decomposition had similar behavior. Remaining constant at $\sim 30 \%$, the three-component decomposition remained independent of inflow velocity. As more energy was added to the system from the cross-flow, the energy contained in the most significant features increased proportional to the total increase. Similarly, the two-component decomposition behaved similarly but only for higher $R_{v}$. For the lowest $R_{v}$ case, the first mode increased, indicating the out-of-plane contributions were found in higher order features.

While the cumulative energy provided insight into the concentration of the turbulence, visualization of individual modes allowed for a more thorough description of the structures within the flow-field. For the relative flow-fields, each component was used to illustrate the largest energy fluctuations moving a) normal to the jet centerline, b) tangential to the centerline and c) entering and exiting the centerplane. These are presented in figures $4.26,4.27$ and 4.28 respectively. The JCB and $\xi^{0}$ was marked to better describe and identify the locations of features.

In Meyer et al. [145], the cartesian coordinate system was decomposed. In the streamwise component, the first mode primarily contained structures upstream of the jet in the cross-flow within the wake region on the lee-side, after the collapse of the core. For successive modes, the features were focused away from the exit along the centerline. A similar result was observed in the present study with the first mode containing large features outside the shear layer both upstream and within the wake region. The most significant features after the first mode described the jet immediately after the collapse of the core around $\xi^{0}$. This was determined to represent the "flapping" of the jet as the core collapsed and the jet became a free- 

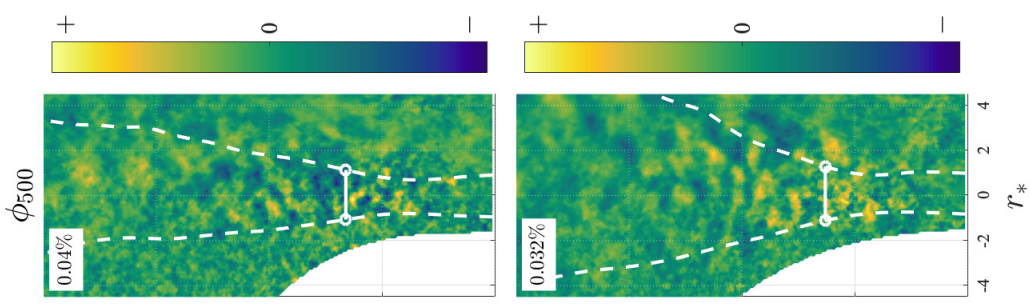

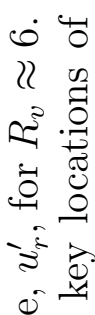
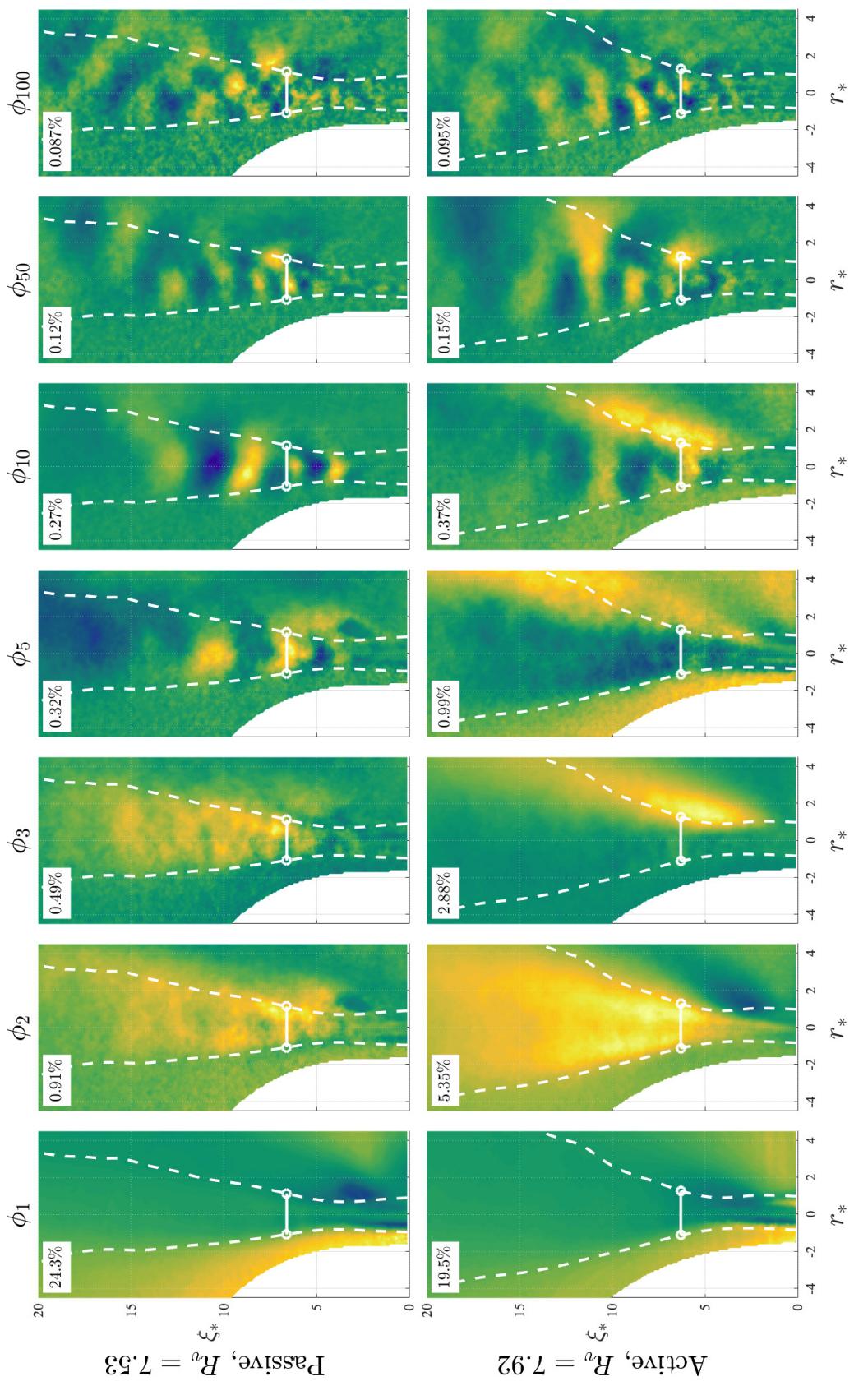

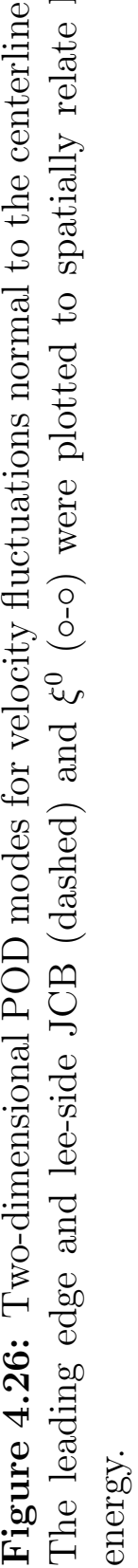


shear flow. For the PG cases, these features contained $\sim 30 \%$ of the energy. For successive modes, the energy was concentrated within the shear layers from $\xi^{0}$ and beyond. These include smaller features that decreased in size until the highest order fluctuations were spread across the entire flow-field.

The first mode of the PG cases encompassed $\sim 25 \%$ of the total energy to describe the flow outside the jet, with the remainder of modes describing smaller features. With turbulent cross-flow, additional features w present that were significant enough to be separated from the first mode. For AG, the first mode included the incoming flow and wake region followed by the jet after $\xi^{0}$. This description of the jet beyond $\xi^{0}$ was interrupted by additional modes that solely described the wake region. As turbulent cross-flow entrained in the wake region, it accounted for $\sim 3 \%$ of the total energy. Additionally, higher order modes included features in the wake region and inflow, indicating higher order fluctuations that were present within the cross-flow. The shear layer expansion was related to the presence of small scale fluctuations. Hence, presence of features at the JCB in higher order modes indicated a significant change in entrainment that increased the jet width.

For the tangential component, $v_{*}^{\prime}$, the PG and AG cases remained similar. The first mode was only present within the jet below $\xi^{0}$ as the shear layers converged and the core collapsed. When coupled with the normal component, the simplest interactions of the jet were described with more than $20 \%$ of the total energy: the energetic inflow, the collapse of the core of the jet and the wake region on the lee-side. The second mode adds to this description and the contributions were concentrated along the leading edge of the jet. This was determined to the be the formation of the jet shear layer vortices along the leading edge. The jet experienced a body force 


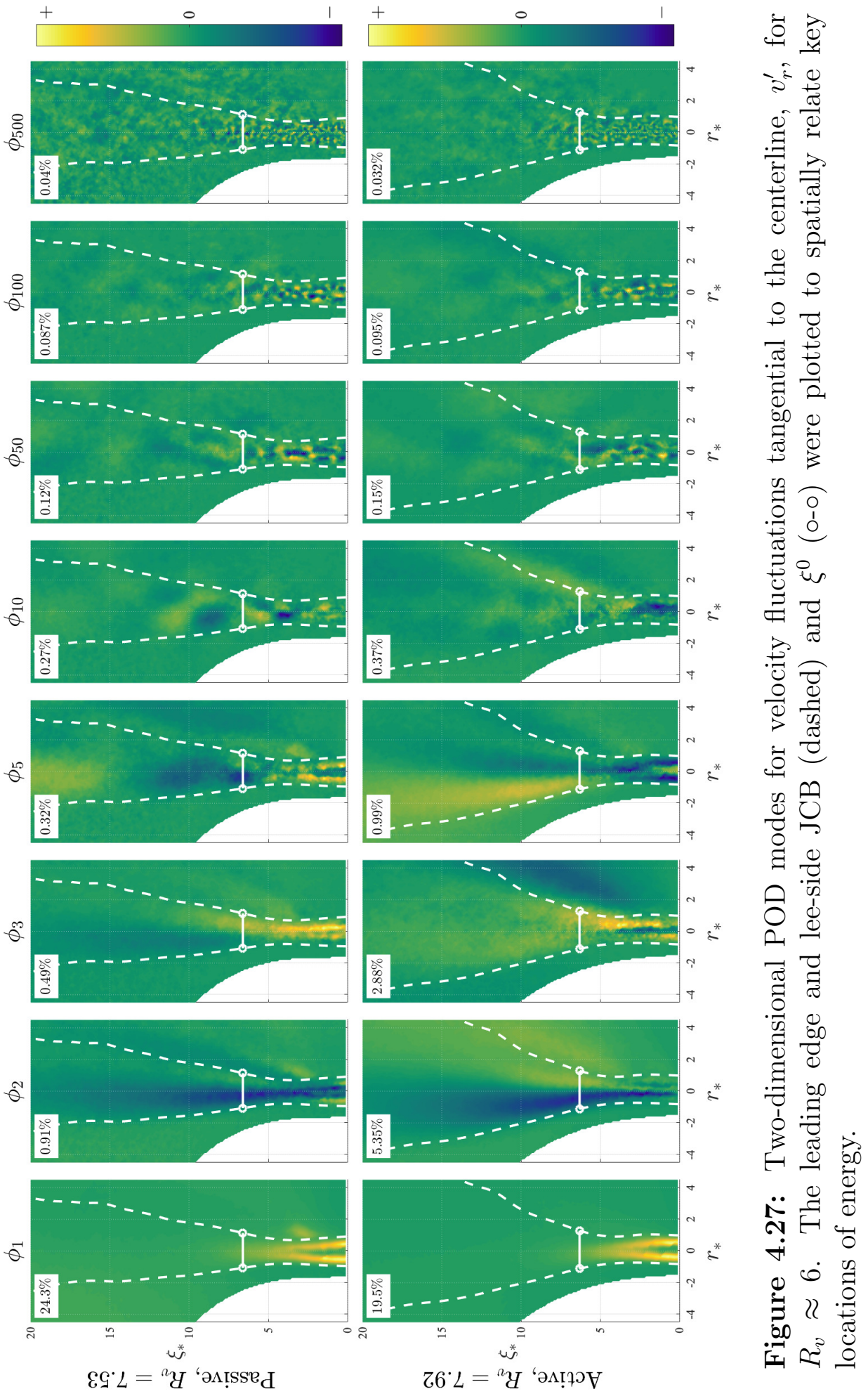


on the leading edge from the cross-flow that created large fluctuations and turbulent energy.

Higher order contributions were primarily focused within the jet before $\xi^{0}$. Within a highly turbulent flow, energy was transferred from the large to smaller scales. Because the tangential component was present only within the jet and in the wake region, the presence of energy contributions at higher modes was the result of this cascade of energy. Comparing the high- and low-turbulent inflow, the tangential decomposition appeared to be similar. While additional modes were focused along the leading edge, this was the result of the smaller turbulent features in the inflow interacting with the turbulent jet.

For the out-of-plane component, the first mode indicated that the inflow turbulence did change the most energetic regions of the jet. For the PG case, energy was concentrated along the shear layers and within the wake region before $\xi^{0}$. This was determined to be the reorientation of the shear layer vortices leading to the formation of counter-rotating vortices (see Kelso et al. figure 9 [59]). On the lee-side, the small region near the exit that changed magnitude identified the location where the orientation of the out-of-plane velocity changed as the cylindrical cross-section changed. Successive modes described the further development as the small vortices that formed on the side of the jet converged on the centerline forming the large counter-rotating vortices.

Comparisons to the AG cases identified changes to the most significant features of the jet. Within the first mode, the most energetic region shifted from lee-side to the leading edge of the jet. While this regions was present in the first mode of PG cases, the absence of the lee-side features indicated a change in wake behavior. This 

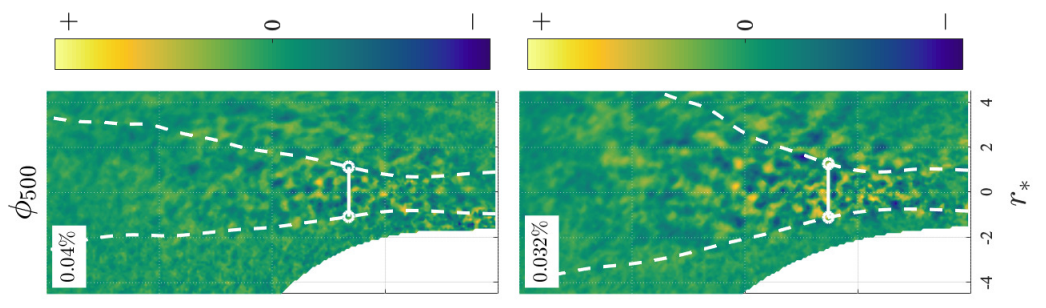

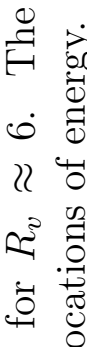
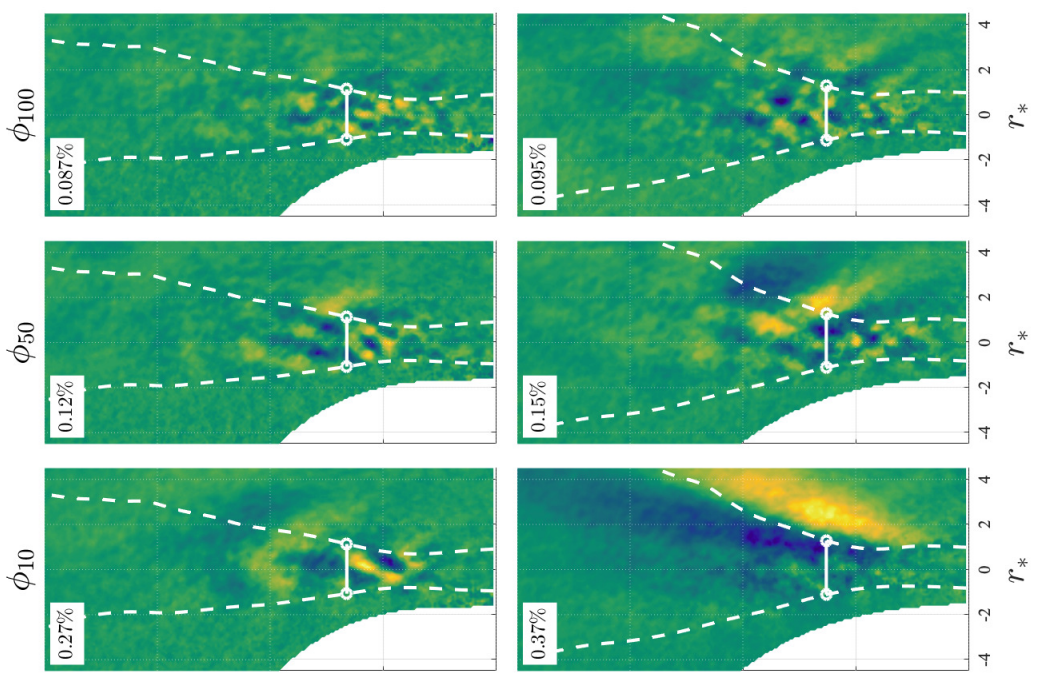

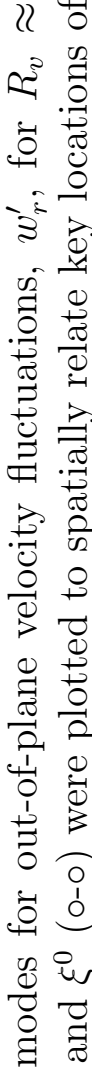
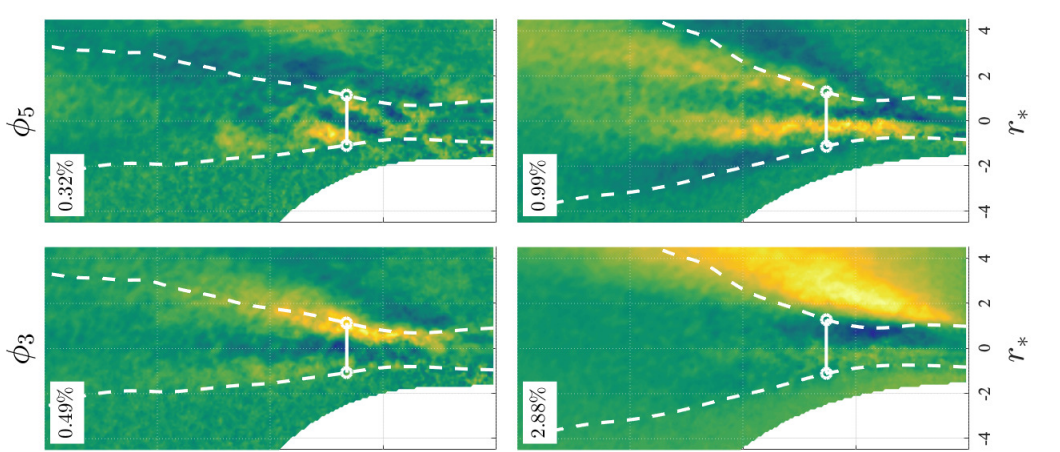

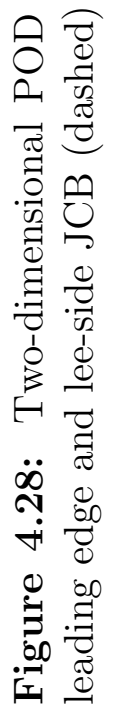
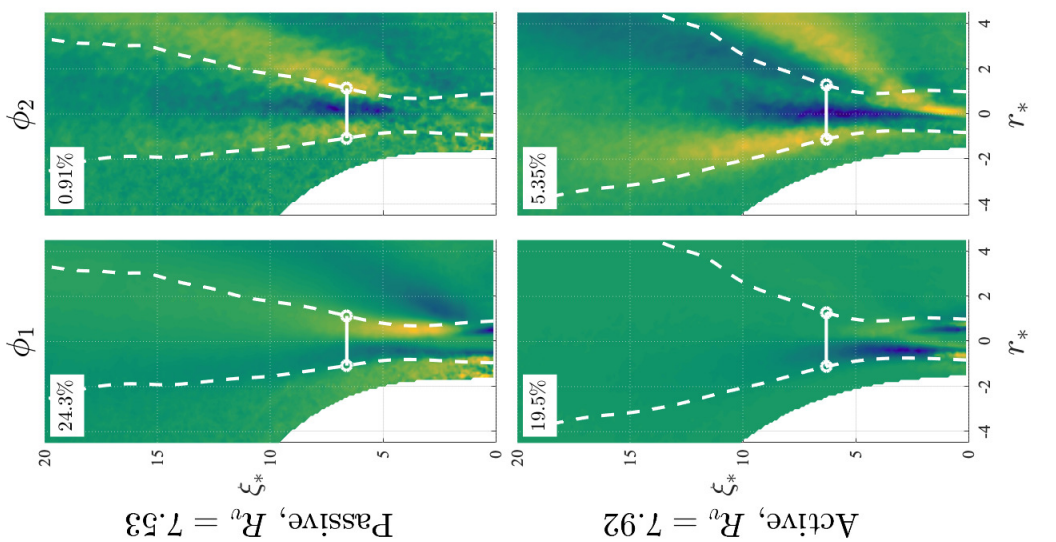
region was not present until successive modes, when both the entire wake region and the lee-side shear layer were included. Therefore, the increased higher order fluctuations of the inflow resulted in a shift from low to higher order contributions.

\subsubsection{Select Mode Reconstructions}

By limiting the number of modes included in the reconstruction of the flow-field, the smallest features were eliminated. It was not trivial to determine the number of modes required to represent the flow field and Reynolds stresses. For this, a range of modes were used to reconstruct the fluctuating field and the Reynolds stresses. For each reconstruction, the JCB was evaluated using the method described in Appendix A.

The quality of the reconstructions were evaluated by identifying how the reintroduction of energetic features distort the JCB. For each case, the cross-sectional area of the jet was computed for each mode from $\xi=\left[0, \xi^{0}\right]$ (Figure 4.29a). As the most significant features were reintroduced, the area of the jet decreased, reaching a minimum and then increasing until the full reconstruction was complete.

From the modes, the location of the most energetic contributions were focused within the jet and the cross-flow, followed by interactions at the JCB. As energy was introduced back into the system, the jet was large at first as the cross-flow was neglected. With the cross-flow reintroduced, the jet narrowed until the jet and cross-flow had been included without the highest order fluctuations. Therefore, an accurate description of the near-field of the jet should be selected when the area has been minimized. Evaluating the final area before the collapse of the core in Figure $4.29 \mathrm{~b}$, the near-field area of the jet was directly related to the velocity ratio 

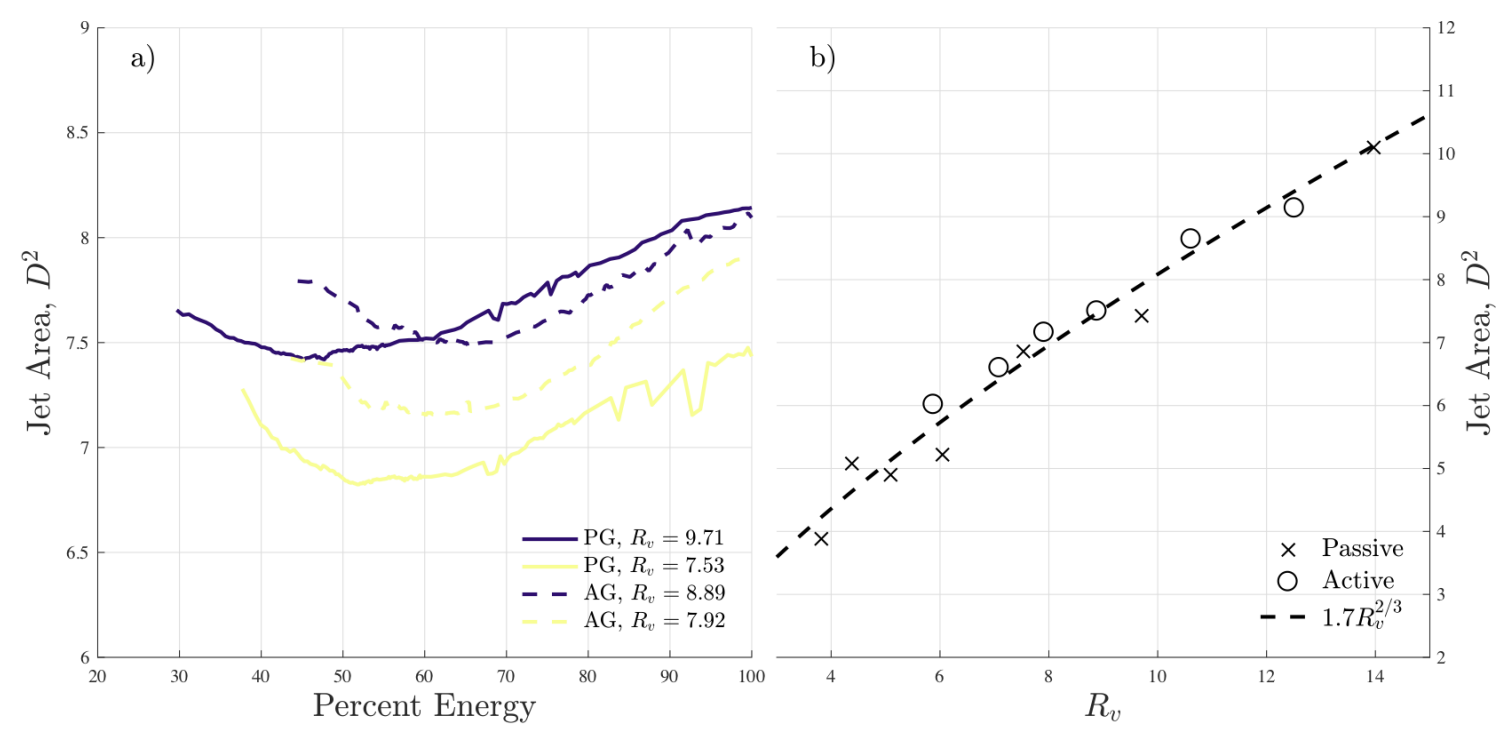

Figure 4.29: Measurement of a) the area of the jet measured from $\xi_{*}=0.1 \rightarrow$ $\xi_{*}^{0}$ for PG (solid) and AG (dashed) and b) the final area of the jet after reconstruction.

and independent of the inflow conditions. The decreased area is a result of increasing body forces from the cross-flow. Identifying these key features distinguished the near-field of the jet as independent of free-stream turbulence.

\subsubsection{Summary}

The jet was decomposed using snapshot POD, which displayed the most energetic flow features. This first identified how the structures were ordered. The most energetic regions were found in the lee-side wake region. Smaller contributions were found where the shear layers converged, with the least energy in random fluctuations. Evaluation of the JCB for reduced order reconstructions change the near-field area, shrinking and expanding as the cross-flow and higher order features were reintroduced respectively. At the smallest area, when only the cross-flow and jet were included, the jet was compressed by the inflow and the addition of free-stream tur- 
bulence did not alter the expansion.

\subsection{Reduced Order Modelling Parameters}

While identifying the changes to the largest coherent structures provides a richer description to large scale engulfment, the small scale viscous nibbling remains unresolved due to the resolution of the PIV data. Thus, an investigation of small scale features was approached to quantify the turbulent features that could not be directly observed. The methodology outlined in section 2.3 was applied to compute and compare the smallest scale features to inform future models.

\subsubsection{Eddy Viscosity}

For LES models, the filtered Navier-Stokes equations account for unresolved components through the turbulent eddy viscosity, $\nu_{T}$. This was computed directly through the linear eddy viscosity model [122]. The gradients were determined using the Akima spline method [151] to reduce strong variations without smoothing values. From Beresh et al. [152], the turbulent eddy viscosity was determined by linearly fitting (2.34) for all known gradients and Reynolds stresses, assuming continuity and out of plane derivatives were negligible.

The simplest form of $\nu_{T}$ is evaluated first using the shear component from (2.35) (Figure 4.30a and d). Along the centerline, $\nu_{T}$ exhibited asymptotic behavior due to $\left\langle u^{\prime} v^{\prime}\right\rangle$ and $S_{12}$ crossing zero, which was similar to those observed in Beresh et al.[152]. Outside the lee-side JCB, artifacts from the PIV processing yielded spikes in $\nu_{T}$. For low inflow turbulence, features such as the wake region and JCB were bordered by large changes in magnitude due to the changing orientation of the flow 

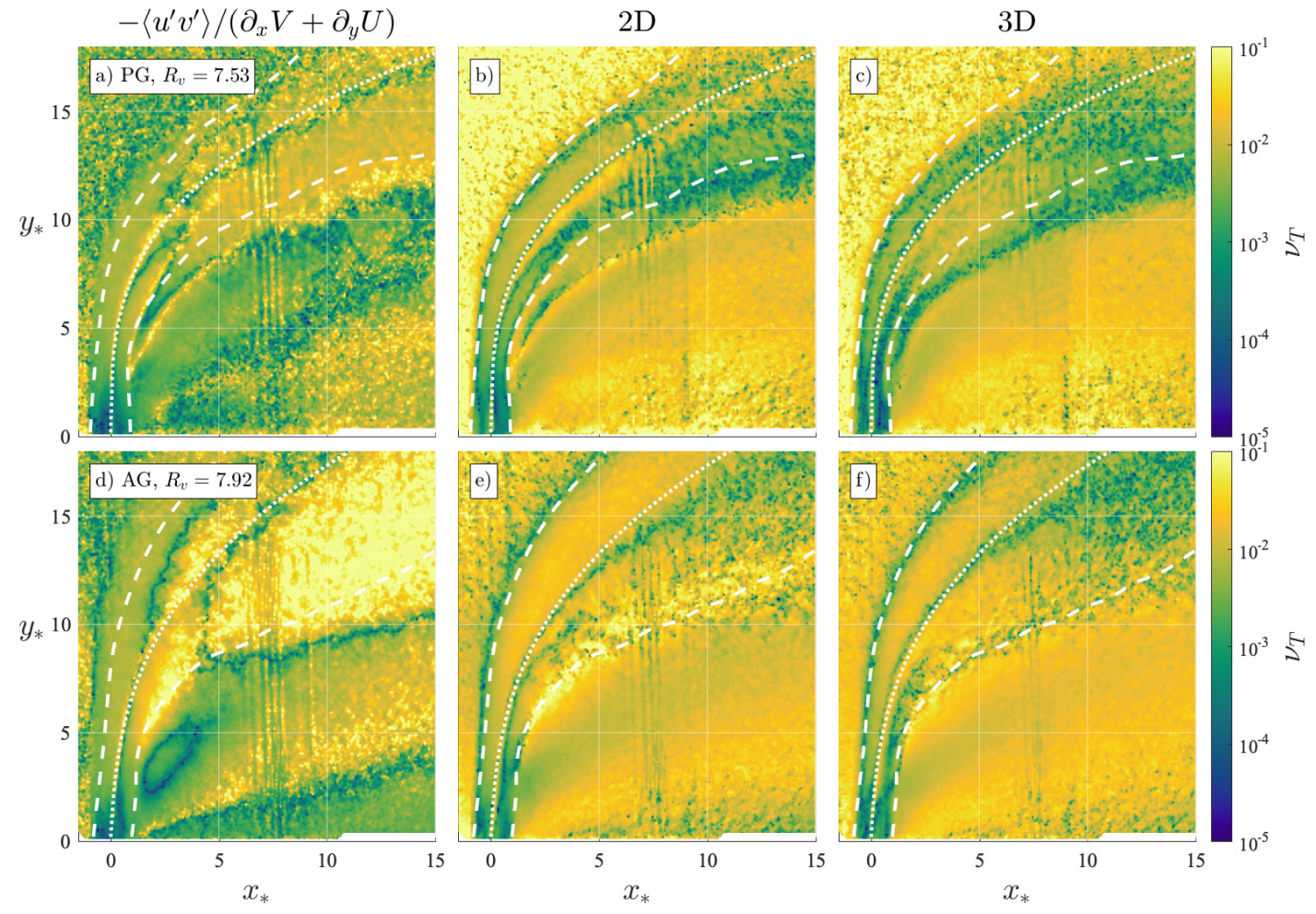

Figure 4.30: Turbulent eddy viscosity determined from the linear viscosity model for PG (a-c) and AG (d-f). The centerline (dotted) and JCB (dashed) are included for visual comparison. The eddy viscosity has been represented on a logarithmic scale to allow for a broader range of values.

relative to the exit conditions. For AG, high magnitude sub-grid contributions were present along the lee-side JCB as the jet bends downstream which extended to the far-field. The recirculation region and a boundary layer profile were bordered by orders of magnitude changes in $\nu_{T}$. The one component solution only described regions of high magnitude sub-grid contributions due to the shear induced by the orthogonal jet and cross-flow. Energy was still contained in other components, therefore consideration of additional contributions was necessary.

Introduction of the remaining components of the two- and three-component lin- 
ear viscosity model removed the effects of inflection points in the gradients. Ignoring out-of-plane velocity, the two-component solution (Figure 4.30, panels b and e) dramatically improved the inflow and wake regions behavior and removed sharp centerline asymptotic behavior. For PG, where out-of-plane movement were higher than similar AG cases, the large variations within the jet were reduced but not optimal. Incorporation of the third component (Figure 4.30, panels $\mathrm{c}$ and $\mathrm{f}$ ) further improved the inflow and lee-side wake region, but only offered minimal improvement within the jet. A drop of $\nu_{T}$ of nearly an order of magnitude was observed along the edge of the shear layer due to changes in the velocity gradients before the Reynolds stresses and zero crossings. Due to the changing orientations of the flow, out-of-plane movement and high anisotropy, it was expected that the linear model will have some shortfalls with the assumptions.

While the linear model provided reasonable ranges to describe unresolved contributions, further simplification was made to reduce the amplifications observed in $\nu_{T}$. The velocity components were transformed from the $x-y$ plane to components normal and tangential to the jet centerline (see Appendix B). The gradients were re-evaluated along this axis to measure changes relative the jets length, $\xi$, and the radial distance off the centerline, $r$. The simplified form of the linear viscosity model (equation (2.35)) was evaluated for the shear component $\left\langle u_{*}^{\prime} v_{*}^{\prime}\right\rangle_{r}$ and strain component $S_{12, r}$. This was chosen to incorporate the shear layer growth and shear layer vortices, regions of high turbulence kinetic energy. Starting from the exit of the jet, the strain components were plotted against the shear stress from the leading edge to lee-side JCB. A linear fit across the resulting profile, as presented in Figure 4.31, allowed $\nu_{T}$ to be described using only the length of the jet. 


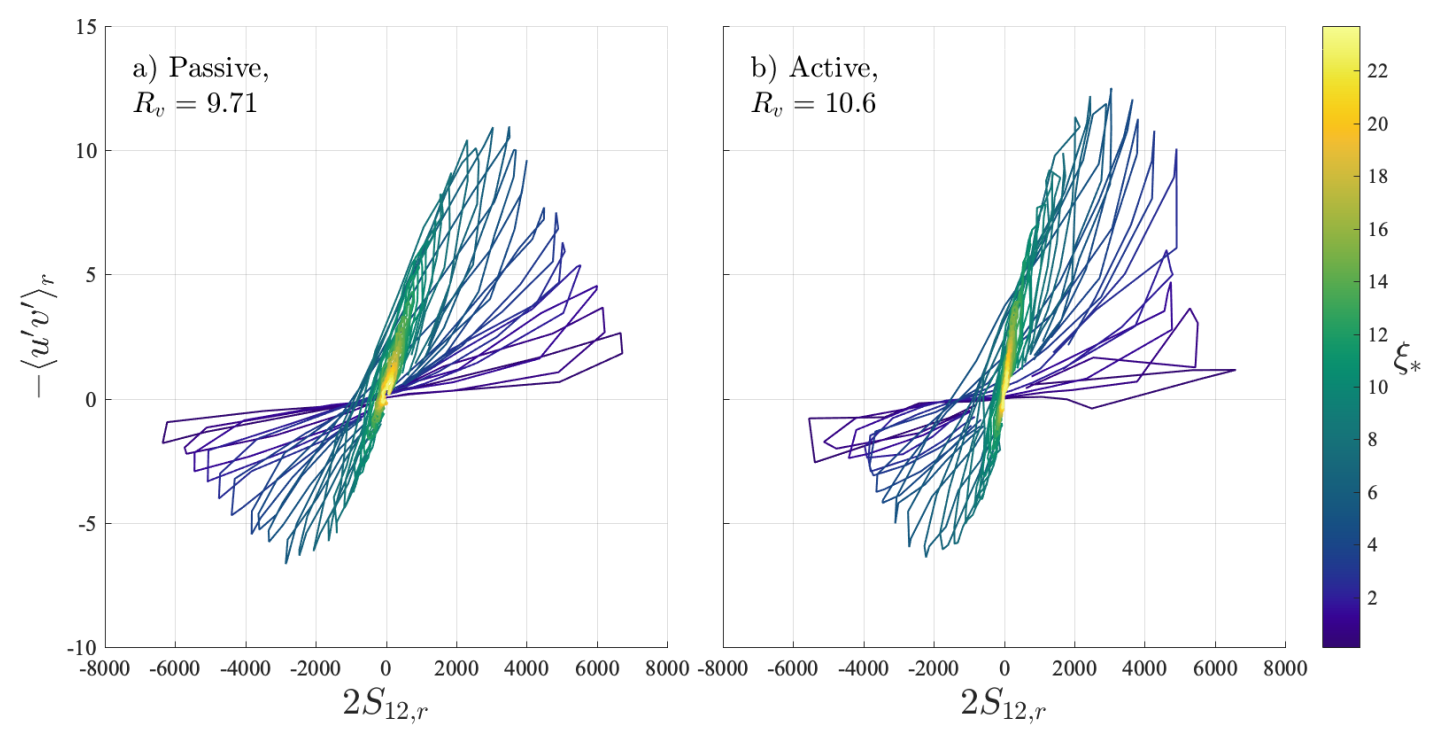

Figure 4.31: The method of evaluating the turbulent eddy viscosity on the relative axis using the shear stress and mean strain rate profile across the width of the jet for one PG and one AG case.

The result of the linear fitting was compared to the linear viscosity model to determine the proximity to more complex approximations. From the results obtained in Figure 4.30, $\nu_{T}$ was evaluated on the jet axis $(r-\xi)$. Scalar values were evaluated at the same locations using bivariate spline interpolation [153]. Averages were taken across the radius of the jet (within the JCB) and plotted as a function of jet length in Figure 4.32. Within the near-field, the linear viscosity model yielded large variations that fluctuated over an order of magnitude. In the far-field, large fluctuations appeared in the one component linear model while the two- and three-components were reduced. The addition of the second and third components increased the subgrid contributions as additional energy was added to system. Evaluation of the shear component on the relative axis across the width of the jet removed the fluctuations yielding a distinct curve that increased from zero at the exit and converged 


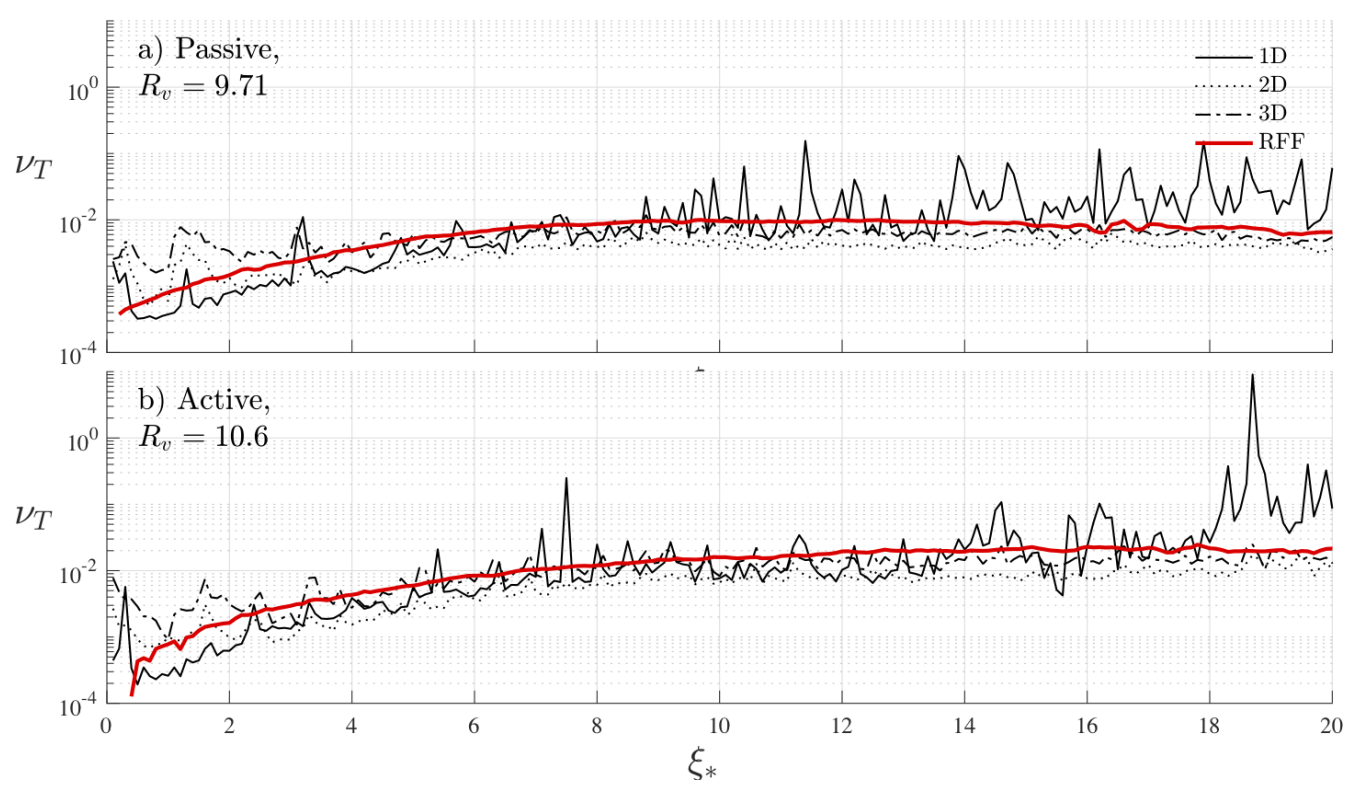

Figure 4.32: Comparison of the turbulent eddy viscosity from linearly fitting the profiles in Figure 4.31 to the mean shear, two- and three-component linear eddy viscosity model within the jet from Figure 4.30.

on a nearly constant value in the far-field. Additionally, the smoothed curve was greater than the two and three-component curves, indicating an increase in sub-grid contributions to the total energy.

Evaluation of $\nu_{T}$ through the linear viscosity assumption created a mean profile across the jet that fluctuated over orders of magnitude. The uncertainty in PIV processing $(\sim 3-5 \%)$ was amplified through the gradients. By fitting a region of the flow that behaves linearly (the width of the jet in this case), variability of $\nu_{T}$ was reduced as a function of the jet length. Near the exit, $\nu_{T} \approx 0$ when the jet was unaffected by the cross-flow. As the jet developed and becomes fully turbulent, $\nu_{T}$ increased. This described an increase in sub-grid contributions that were unresolved on the PIV grid. Once a peak was achieved, the gradual decrease in $\nu_{T}$ indicates more energy was dissipated than transferred from larger eddies. 

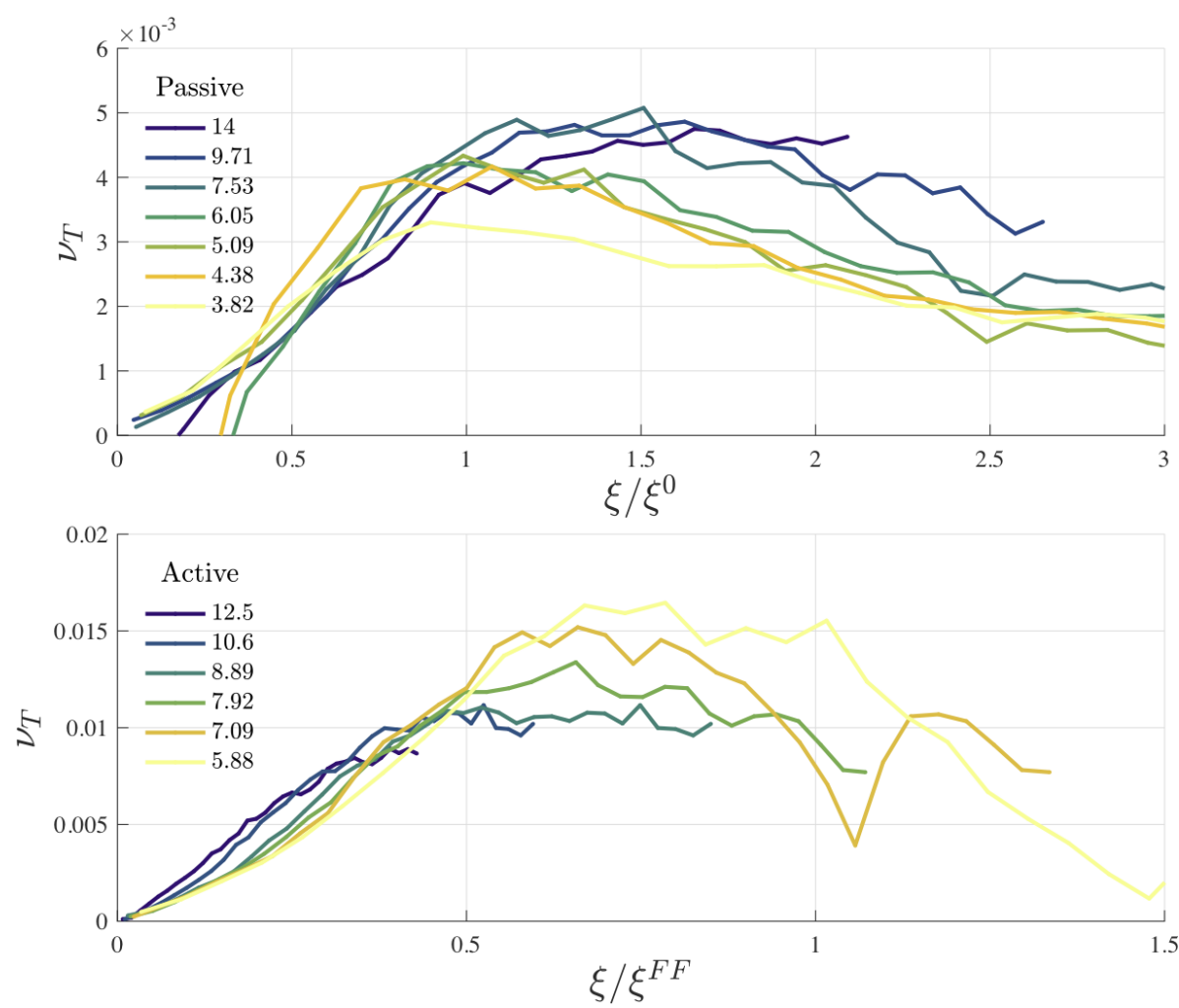

Figure 4.33: Eddy viscosity as a function of the scaled jet length $\xi / \xi^{0}$ for low inflow turbulence (top panel) and $\xi / \xi^{F F}$ for high inflow turbulence (bottom panel).

Similar to the other features, a comparison between cases as a function of jet length did not collapse the curves. From Freedland et al. [154], the collapse of the potential core as the shear layers converged was independent of inflow turbulence and followed an empirical relationship. This location, $\xi^{0}$, was used to scale the jet length as $\xi / \xi^{0}$ (top of figure 4.33) for the PG cases. The sub-grid contributions increased before $\xi^{0}$, peaked within the range of $0.003 \leq \nu_{T} \leq 0.005$ and decreased through the remainder of the jet. This described the dominant physical processes that contributed to the growth of the jet. Entrainment can occur on both large and small scales through the processes of engulfment and viscous nibbling respectively. 
As the jet developed, the small scale features within the shear layer entrained an increasing magnitude of energy. After the collapse of the core, the small scale features decreased as the counter-rotating vortices formed and the energy is primarily contained in the largest eddies.

In high turbulence inflow, sub-grid contributions continued to grow after the collapse of the core. The small scale fluctuations in the cross-flow were entrained and the expansion of the shear layer increased. This added additional small scale features through viscous nibbling yielding a converged $\nu_{T}$ nearly four times the peak of the PG cases. Therefore the far-field transition point, $\xi_{*}^{F F}=0.3 R_{v}^{2}[31]$, was chosen to scale the jet length (figure 4.33b). Approaching this location, the sub-grid contributions reach a peak at $\xi / \xi^{F F}=0.5$ and then drop off significantly. From the out-of-plane component, the absence of out-of-plane motion indicated the counterrotating vortices are not present. Therefore, the large vortices are no longer present and the increased expansion within the shear layer is comprised of small scale contributions. It should be noted that this result did not provide an exact quantification of $\nu_{T}$. It did provide a qualitative initial comparison between inflow conditions and identified the importance of sub-grid contributions in simplified models and the influence of free-stream turbulence.

\subsubsection{Dissipation (Modified Structure Function Method)}

The ability to determine correct magnitudes of dissipation despite limitations in spatial resolution provided the most benefit to optimizing modelling parameters. As outlined in Chapter 2.4, the dissipation could be computed through its definition $\varepsilon \equiv 2\left\langle s_{i j} s_{i j}\right\rangle$ with the assumption of incompressibility and divergence-free continuity 
which yielded (2.49). For experimental techniques such as PIV, direct calculation of this parameter was inaccurate. Similar to the Nyquist rate in signal processing, a significantly lower resolution incorrectly predicted fluctuating gradients. When squared, this error was amplified and could lead to an under-prediction in dissipation by more than an order of magnitude [155].

The gradients of the fluctuating velocity components were evaluated over each snapshot through a five point slope differencing method [151]. The resulting measured gradient dissipation, $\varepsilon_{g}$, was determined by solving (2.49) directly and presented in Figure 4.34.

The greatest dissipation was concentrated within the core of the jet regardless of inflow conditions. The most energetic regions (similar to the TKE) was focused along the shear layer near the floor, converging and expanding, then decreasing in magnitude through the remainder of the jet. As observed in the results of Miller et al.[156], $\varepsilon \sim \mathcal{O}\left(10^{6}\right)$, while the computed gradients are almost two orders of magnitude lower. This was corrected by relating the dissipation to the second order structure function $D_{L L}$ through Kolmogorov's two-thirds law [34]. If the grid spacing was equivalent to the smallest scales, then the measured structure function dissipation, $\varepsilon_{s, m}$, would be equivalent to the true dissipation. As the grid spacing was increased, the accuracy exponentially decreased [6]. Utilization of the Modified Structure Function Method corrected the dissipation through empirical relationships determined between DNS data and a filtered lower resolution form (see full description of method in Chapter 2.4). This yielded both the true dissipation and the smallest scales within the flow.

From (2.50), the second order structure function was defined over two locations, 

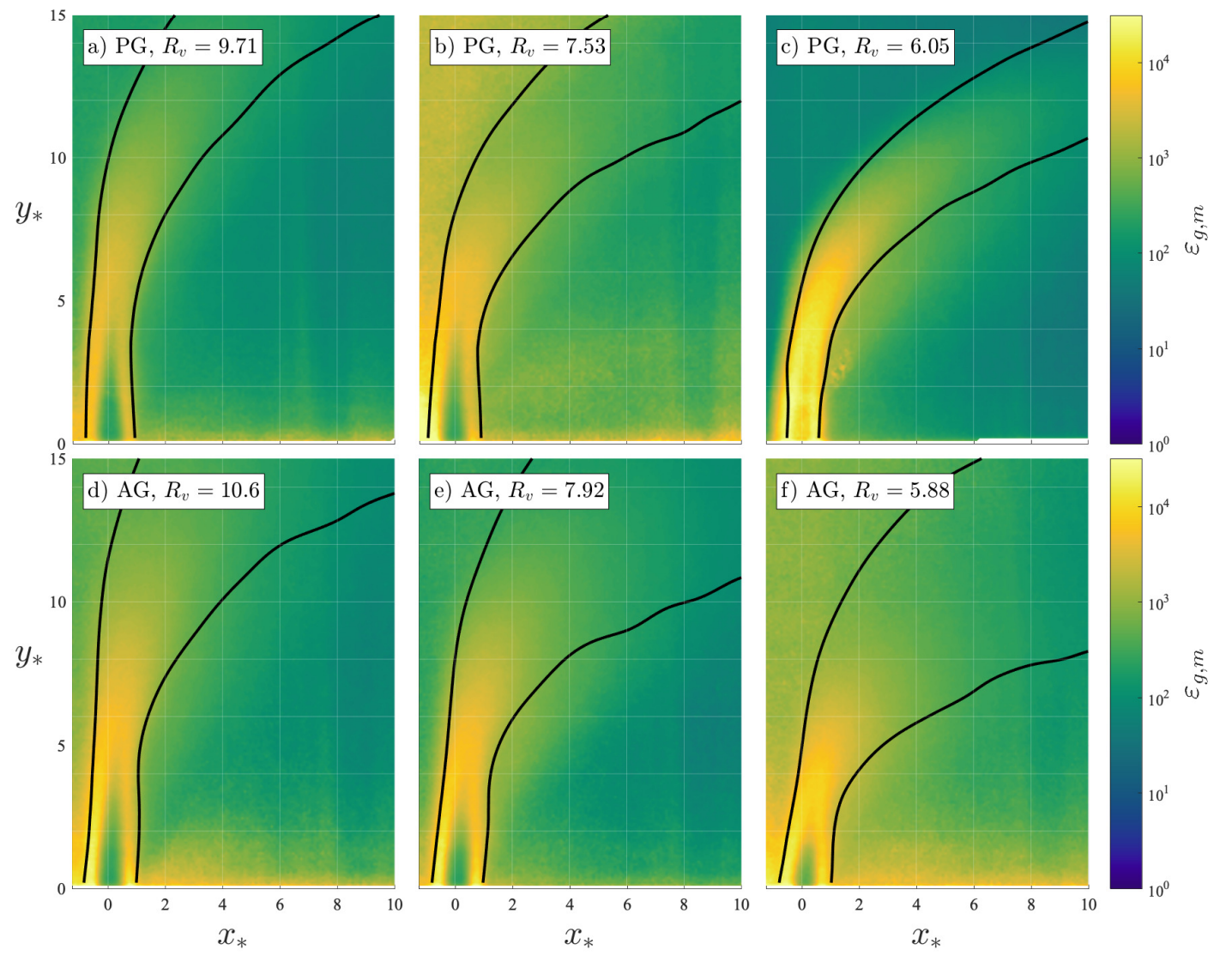

Figure 4.34: Turbulent dissipation calculated directly as $\varepsilon=2 \nu\left\langle s_{i j} s_{i j}\right\rangle$ for three low (a-c) and three high (d-f) cross-flow turbulence intensities.

$\mathbf{x}$ and $\mathbf{x}+r$. The offset, $r$ was best defined in the direction of fluid motion to fully capture the turbulent features. The cross-flow could be assumed quasi onedimensional and was evaluated without any additional reorientation of the flow. Within the jet and wake region, the flow was multi-directional. The bending motion within the jet and unstable focus within the wake region created an additional level of complexity. Therefore, to evaluate $D_{L L}(\mathbf{x}+r)$ in only one dimension, the path of a particle at location $\left(x_{0}, y_{0}\right)$ is determined and $r$ would evaluated along it. For each case, the flow-field was divided into three regions. The inflow (I) was evaluated 

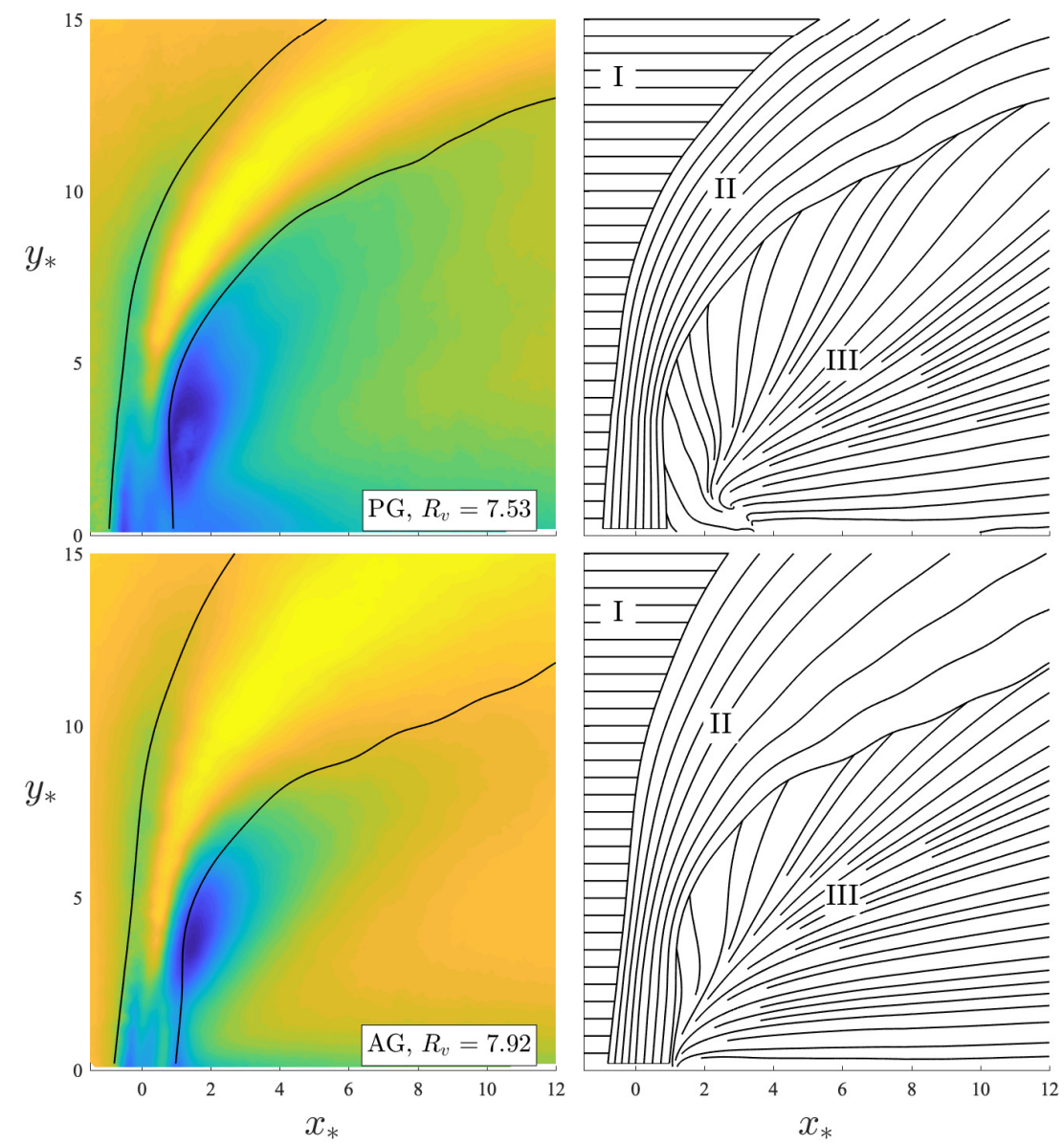

Figure 4.35: Separated regions of the flow-field and streamlines for evaluation of $D_{L L}$. The inflow (I) was evaluated using streamwise component, $u$. The jet (II) was evaluated using the velocity component tangential to the centerline, $v_{r}=u \sin \left(\theta_{C L}\right)+v \cos \left(\theta_{C L}\right)$. The wake region (III) was evaluated in the direction of the flow at the current location, $v_{r}=u \sin \left(\theta_{\phi}\right)+v \cos \left(\theta_{\phi}\right)$.

downstream $(r=r(x))$ to account for streamwise turbulence. The jet (II) was evaluated on the same axis as $\nu_{T}$, with vectors tangential to the centerline, $r=r(\xi)$. Within the wake region (III), a streamline $\phi(\mathbf{x}, \tau)$ was defined as originating at location $\left(x_{0}, y_{0}\right)$ and would be tangential to the magnitude of the velocity. The time offset, $\tau$, was defined to extend the streamline using the velocity components $\bar{U}$ and 
$\bar{V} . D_{L L}$ was evaluated along the full streamline $\left(r=r_{\phi}\right)$ with the velocity component tangential to the streamline. The regions were separated using the defined JCB for each case. A schematic of this method is presented in Figure 4.35.

The dissipation for each region was distinguished with the corresponding numerical superscript: $\varepsilon^{I}$ for the inflow, $\varepsilon^{I I}$ within the jet, and $\varepsilon^{I I I}$ within the wake region. Once $\varepsilon_{s, c}$ was determined for each region, the flow-field was rebuilt to visually identify regions of significant turbulent energy. Three cases for PG and AG are compiled in Figure 4.36 .

The dissipation was found to be largest within the jet primarily along the leading edge JCB where shear layer vortices have been found. As the shear layers converged and the potential core collapsed, $\varepsilon_{s, c}$ peaked within the jet and began to decrease downstream. This highlighted the transition to a fully turbulent jet. Within the wake region, the influence of the unstable focus created a significant drop in $\varepsilon_{s, c}^{I I I}$ surrounding the entrainment region. While a clear boundary was defined in the mean velocity, this boundary was not static. An evaluation of $D_{L L}$ would yield a negative correlation that averaged near zero.

The corrected gradients, $\varepsilon_{g, c}$, was obtained through the correction factor $\beta$, defined as $\beta=\varepsilon_{s, c} / \varepsilon_{g, m}$ (Figure 4.37). Within the jet, the gradients under-predicted the dissipation by an order of magnitude in the near field. This was an expected result due to the unresolved fluctuating gradients and high turbulence kinetic energy. Both upstream of the jet and downstream in the wake region, in regions with low turbulence kinetic energy, the gradients over-predicted the dissipation by more than an order of magnitude. Similar to the problems observed in the linear eddy viscosity model, this error was most likely the result of the assumption of local isotropy. By 

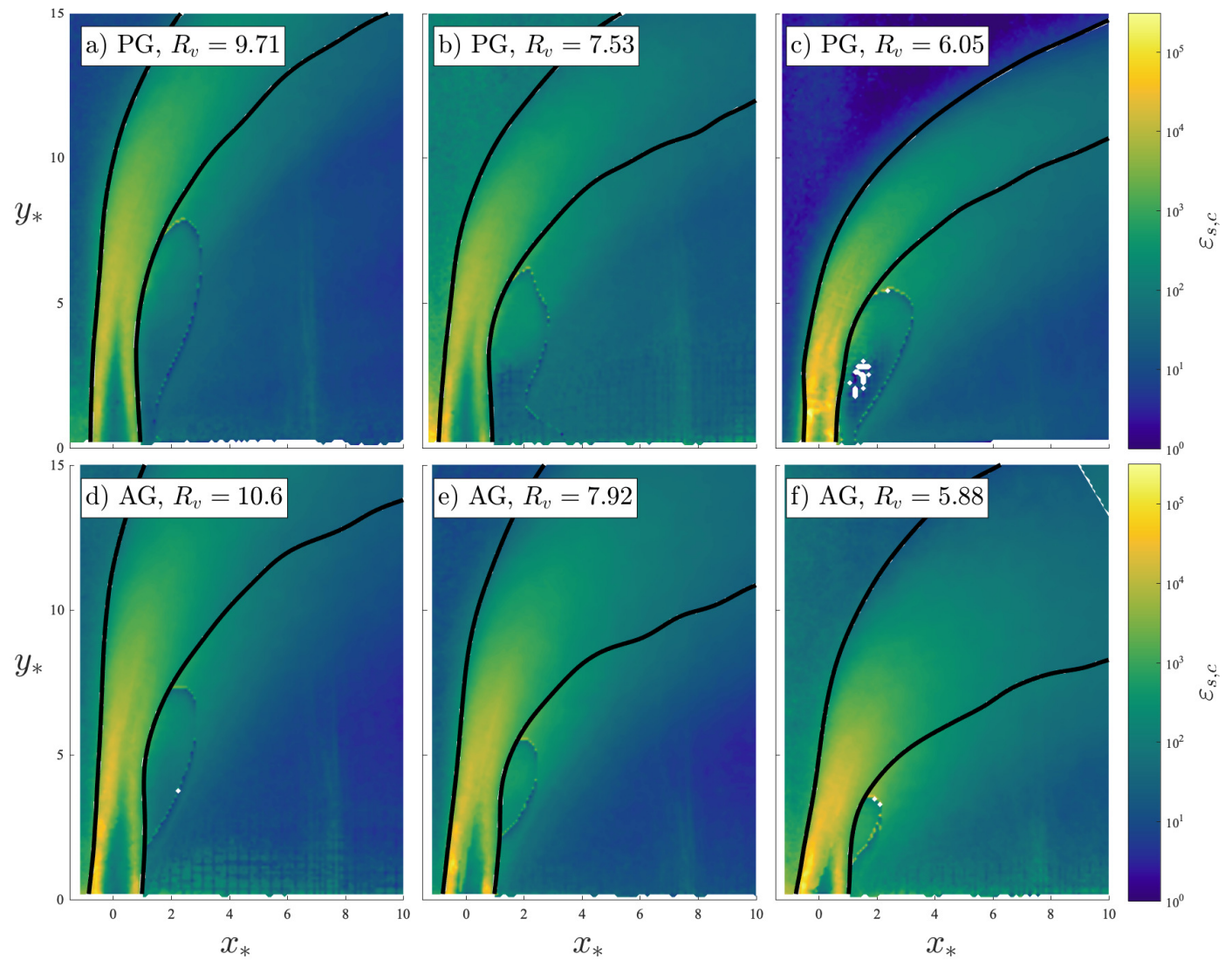

Figure 4.36: Corrected dissipation within each region determined through second order structure functions, $D_{L L}(\mathbf{x}+r)$. The inflow $(\mathrm{a}, \mathrm{d})$ was evaluated downstream $r\left(x_{*}\right)$, the jet (b, e) was evaluated tangential to the jet centerline $r\left(\xi_{*}\right)$, and the wake region $(\mathrm{c}, \mathrm{f})$ was evaluated using streamlines $r\left(\phi_{*}\right)$.

replacing unresolved gradients with known components, contributions that should have been negligible were incorporated into the solution.

While confirmation of these results required resolved data of $\mathcal{O}(\Delta / \eta) \sim 1$, a comparison to the turbulent production could determine if the correct order of magnitude is achieved. The production, $\mathcal{P}$, defined as

$$
\mathcal{P}=\overline{u_{i}^{\prime} u_{j}^{\prime}} \frac{\partial U_{i}}{\partial x_{j}}
$$



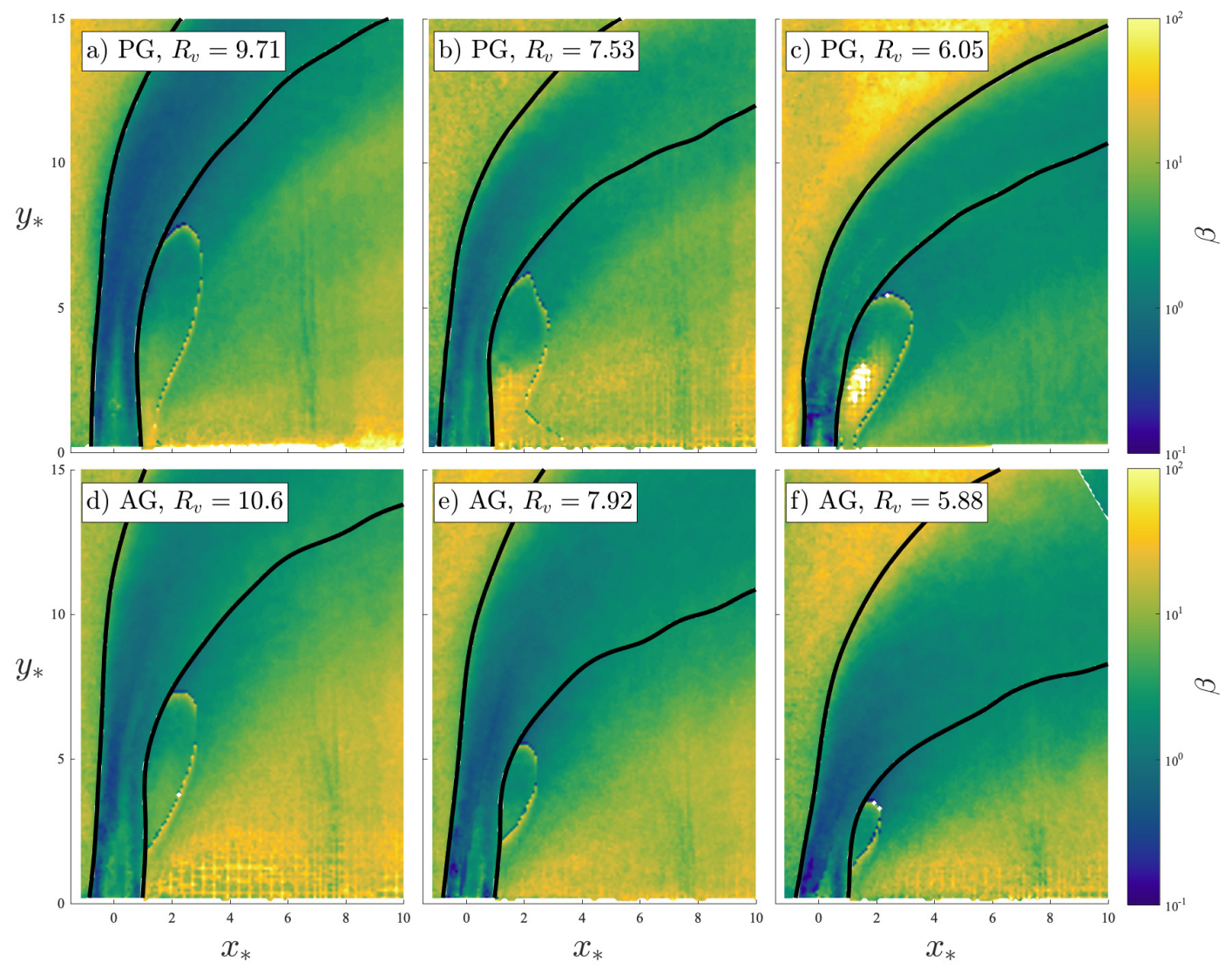

Figure 4.37: Gradient dissipation correction factor, $\beta=\varepsilon_{s, c} / \varepsilon_{g, m}$, for several cases.

should be equivalent to the dissipation $(\mathcal{P} / \varepsilon \approx 1)[30]$. The largest component of the production was chosen to compare to the dissipation within each region. To reduce complexity, $\mathcal{P} / \varepsilon$ was averaged to observe changes over a single axis. For the inflow (I), the streamwise shear index $i=1, j=1$ was dominant due to the quasi onedimensional of the cross-flow with variations in $\varepsilon$ and $\mathcal{P}$, a result of the boundary layer (Figure 4.38a and d). Thus, the field was averaged to observe $\left\langle\mathcal{P} / \varepsilon_{s, c}^{I}\right\rangle\left(y_{*}\right)$. Within the jet (II), the vertical shear component $i=2, j=2$ is dominant due to the turbulent jet. The same approach from Chapter 4.4.1 was applied. Averaging across 


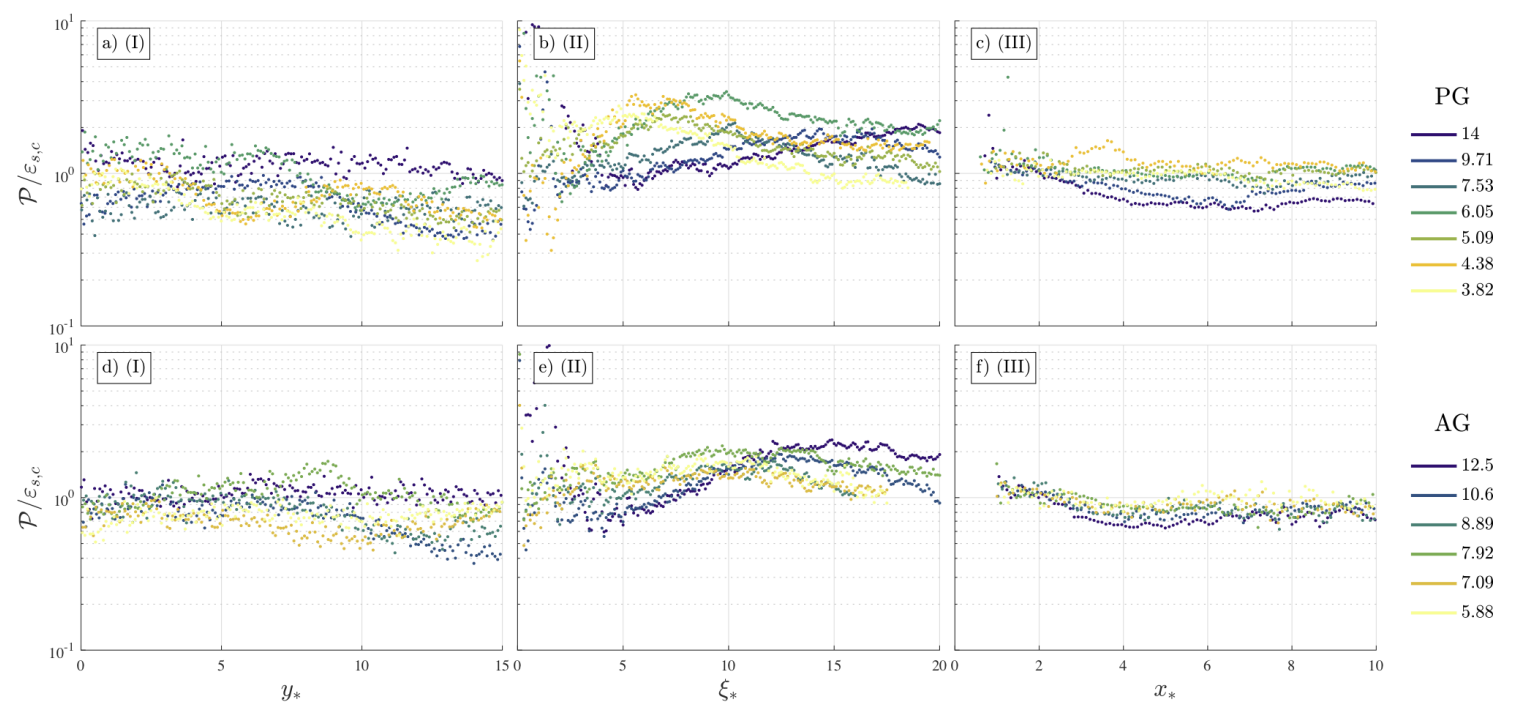

Figure 4.38: The average ratio of turbulent production $\mathcal{P}$ to turbulent dissipation, $\varepsilon_{s, c}$ within three regions. Region $\mathrm{I}$ is averaged in the streamwise direction and plotted as a function of height, $y_{*}(\mathrm{a}, \mathrm{d})$. Region II is averaged across the width of the jet and plotted as a function of jet length, $\xi_{*}(\mathrm{~b}, \mathrm{e})$. Region III is averaged vertically and plotted as a function of downstream location, $x_{*}$.

the width of the jet allowed variations to be observed as $\mathcal{P} / \varepsilon_{s c}^{I I}\left(\xi_{*}\right)$ (Figure $4.38 \mathrm{~b}$ and e). Within the wake region (III), all components of turbulent production were computed and the greatest contribution was compared. The ratios were averaged vertically to observe changes in $\mathcal{P} / \varepsilon_{s c}^{I I I}\left(x_{*}\right)$ moving downstream within the wake (Figure $4.38 \mathrm{c}$ and $\mathrm{f}$ ).

For all regions, the dissipation and production were balanced within an order of magnitude. The inflow and wake region remained nearly constant throughout the flow-field, fluctuating around $\mathcal{P} / \varepsilon_{s c}^{I, I I I} \sim 1$. Therefore, the MSFM was able to accurately resolve the dissipation despite the large PIV grid spacing. Within the jet, where significant turbulent energy was present across a large range of scales, $\mathcal{P} / \varepsilon_{s c}^{I I} \sim 1$. Near the exit, the high variability was a result of low dissipation within the potential core. After the collapse, the dissipation and production became 


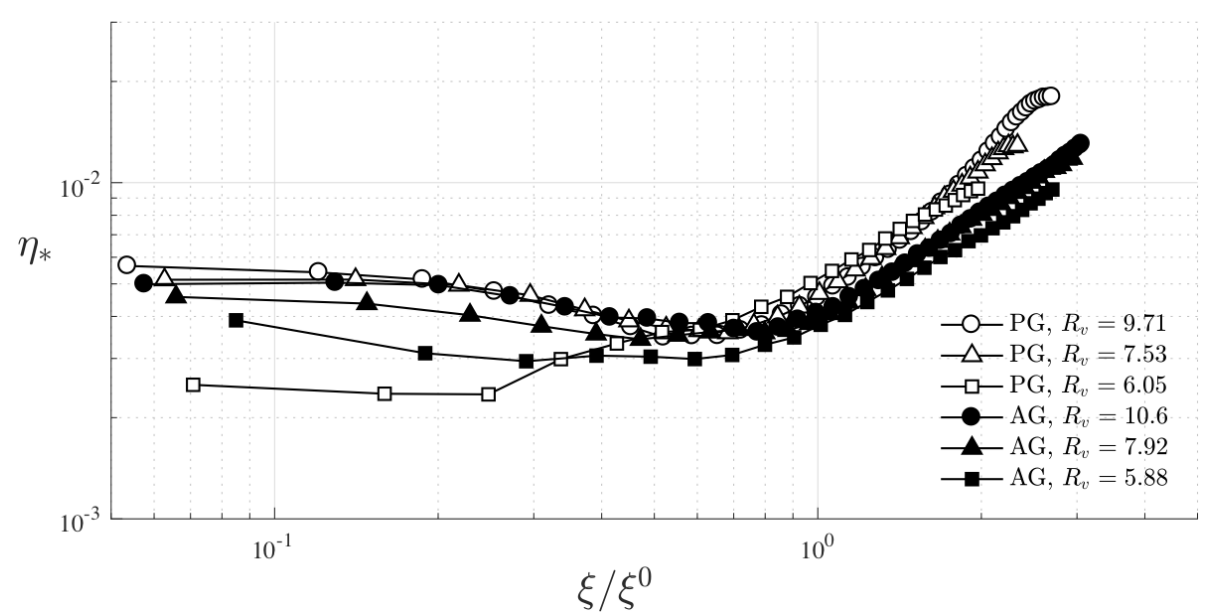

Figure 4.39: The Kolmogorov length scale in diameters, $\eta_{*}$, determined by the MSFM. The scale was averaged across the width of the jet and plotted as a function of scaled jet length $\xi / \xi^{0}$.

unbalanced, with $\mathcal{P} / \varepsilon_{s c}^{I I} \sim 2-5$. Occurring independent of inflow conditions, this imbalance identified an under-prediction of $\varepsilon_{s, c^{\prime}}^{I I}$.

\subsubsection{Kolmogorov Length Scales}

An additional property obtained through the Modified Structure Function Method was $\Delta / \eta$, where $\Delta$ is the PIV resolution and $\eta$ is the Kolmogorov length scale. In Figure $4.39, \eta$ was plotted for three similar $R_{v}$. In the near-field of the jet, the smallest scales were more than two orders of magnitude smaller than the jet. As the shear layer developed and the turbulent production from Figure 4.14 reached a peak $\left(\xi / \xi^{0} \sim 0.5\right)$, the smallest turbulent features were present. After the collapse of the core, $\eta$ increased exponentially.

The exponential increase in $\eta$ occurred at a constant slope which varied for different inflow conditions. As energy was entrained at larger scales, it is cascaded down to the smallest scales and dissipated through viscosity [30]. The larger slope for the 
PG cases indicated that the unresolved energy dissipation was higher, a trend previously observed in the turbulent eddy viscosity (from Figure 4.33). The unresolved energy contributions converged to a constant $\nu_{T}$ as the net energy did not change. The reduced slope for AG occurred due to the additional sub-grid contributions in the cross-flow entrained by the jet. This length scale will be beneficial for future work and will be compared with turbulent features in future sections.

\subsubsection{Modelling Closure Terms}

Utilizing the simplified model of turbulent eddy viscosity and corrected dissipation, closure terms for LES models were extracted. As outlined in Chapter 2.3, models such as $k-\varepsilon$ balanced physical properties using constant coefficients to summarize sub-grid contributions. While these terms were well constrained, the dependence on additional terms in specific conditions complicated the calculation of the flows in varying states of turbulence. Therefore, a direct computation of different closure terms across different regions of the flow was performed. The flow-field was divided into the three regions, as shown in the schematic shown earlier in Figure 4.35. The inflow (I), jet (II), and wake (III), were described as a function of a common length scale.

Beginning with the jet, the most energetic feature of the flow, the closure term, $C_{\mu}$ was determined within the jet through the $k-\varepsilon$ eddy viscosity model, $\nu_{T}=$ $C_{\mu} k^{2} / \varepsilon[78]$. Using the simplified description of $\nu_{T}$ from Chapter 4.4.1, the corrected dissipation $\varepsilon_{s c}^{I I}$ and the turbulence kinetic energy (TKE), $k=u_{i}^{\prime} u_{j}^{\prime}$ from the mean flow statistics to described the jet.

The dissipation and TKE were not constant profiles because of the shear layer 

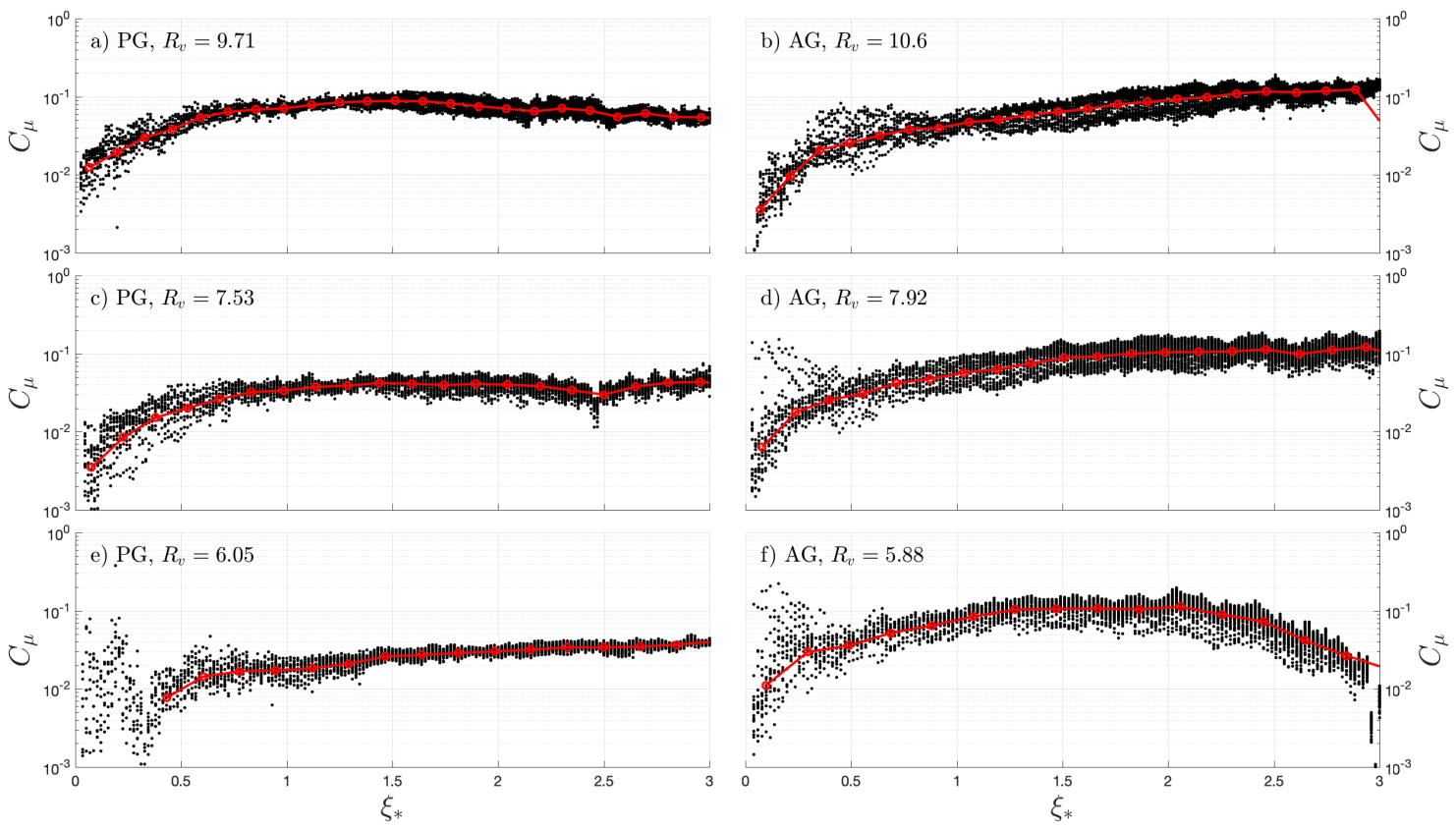

Figure 4.40: Closure argument $C_{\mu}$ determined from the definition $\nu_{T} \equiv$ $C_{\mu} k^{2} / \varepsilon$.

growth and absence of turbulent energy along the centerline (see Figure 4.36). Therefore, a simplified description, presented in Figure 4.40, was used by averaging the values across the width of the jet and over increments of $\xi_{*}=1$. Within the near field, the variations in $C_{\mu}$ spanned nearly an order of magnitude. Physically, this described the high turbulent kinetic energy present as vortex systems began to form and the energy had not been dissipated. When scaled by $\xi^{0}$, the range of values was contained within a small range of values. For PG, the mean converged to nearly a constant value while it continued to grow for AG. Similar to the turbulent eddy viscosity, the convergence observed in the PG cases was a result of a constant ratio of TKE and $\varepsilon$ within the jet. Energy contained in the larger scales was dissipated without additional energy added at smaller scales. Therefore, 

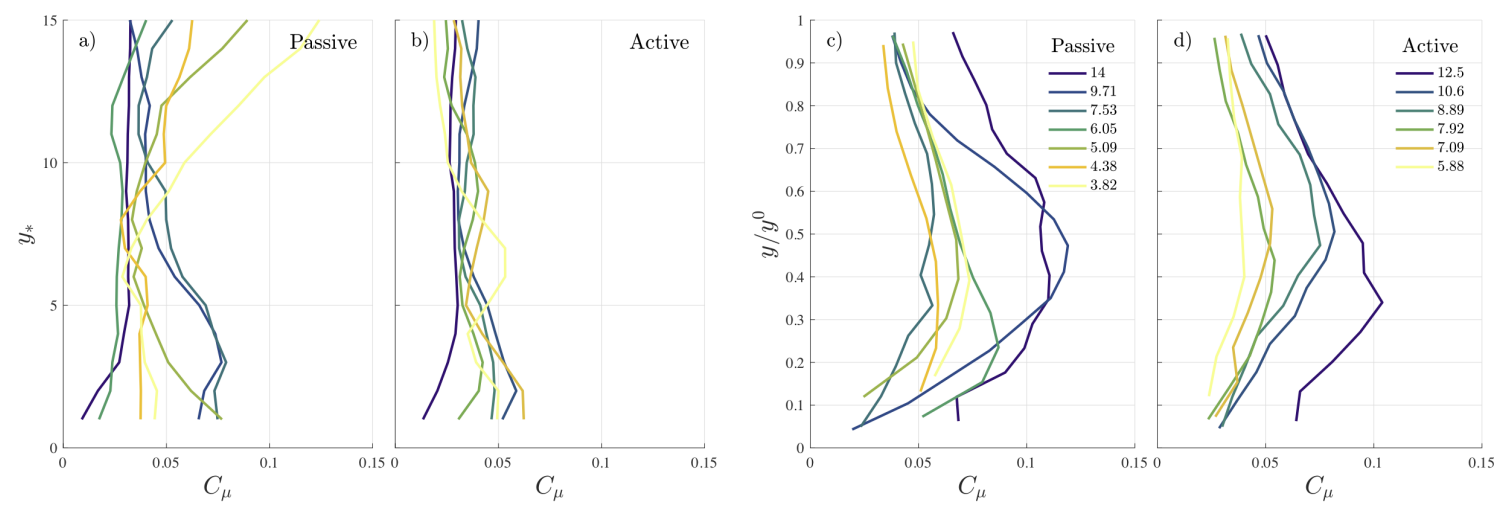

Figure 4.41: Closure argument $C_{\mu}$ determined from the definition $\nu_{T} \equiv C_{\mu} k^{2} / \varepsilon$ in inflow, averaged as a function of elevation $y_{*}(\mathrm{a}, \mathrm{b})$, and the wake, averaged downstream to evaluate as a function of $y / y^{0}(\mathrm{c}, \mathrm{d})$.

additional considerations were not necessary in the far-field. For AG, the additional small scale turbulent energy contained within the cross-flow was observed in the dissipation but not the TKE. Clearly, additional energy must be accounted for until it is fully developed in the far-field.

Away from the jet, the inflow (I) (from Figure 3.4 in Chapter 2) was described as a fully developed boundary layer with low and high turbulence intensity. In Figure 4.41a and $\mathrm{b}$, the profiles ranged between $0.02<C_{\mu}<0.1$ for PG and $0.02<C_{\mu}<0.06$ for AG. Acknowledging the dissipation was accurate within an order of magnitude, and the resulting values agreed with closure terms of $C_{\mu}=0.09$ for free-stream turbulence [78]. The wake region (III) contained a boundary layer that extended downstream and was averaged to evaluate as a function of $y_{*}$. The wake formation was primarily present before the collapse of the potential core. The height at $\xi^{0}$ was defined as $y^{0}$ and was used to normalize the height. In Figure $4.41 \mathrm{c}$ and d, a similar profile across cases peaked within $0.3<y / y^{0}<0.5$. 


\subsubsection{Summary}

In this section, the unresolved contributions and reduced order model closure terms have been directly computed. An investigation of the turbulent eddy viscosity term used in sub-grid models was quantified using the linear viscosity model, assuming continuity and negligible out-of-plane derivatives. Predicted asymptotic behavior along the centerline and JCB was present in the single shear component. Improvements noted by Beresh et al. [152] and Miller et al. [156] were applied for both twoand three-component solutions. The introduction of the third component amplified the shortfall of the method when the vortex systems present in the flow with significant out-of-plane gradients were ignored.

The turbulent eddy viscosity was instead evaluated on the relative flow-field for the shear component $\left(\left\langle u^{\prime} v^{\prime}\right\rangle_{r}\right.$ and $\left.S_{12, r}\right)$. The linear viscosity model was evaluated over the width of the jet, yielding a converged solution defined as a function of the jet length. A fundamental description of the unresolved contributions were determined by scaling the jet length by $\xi^{0}$ and $\xi^{F F}$ for PG and AG respectively. The additional small scale energy present within the cross-flow was entrained by the jet, increasing $\nu_{T}$ until it was fully developed and self-similarity returned.

Sub-grid contributions were known to transfer down scales (through the energy cascade) until they were dissipated at the Kolmogorov length scale. With this in mind, the smallest scales were then investigated. The dissipation, $\varepsilon$, was first computed directly through the fluctuating gradients, which was known to be inefficient, and corrected through the Modified Structure Function Method. The second order structure functions were evaluated along streamlines to account for non-uniform flow orientation. The results yielded significant improvements in predicted dissipation 
that balanced the turbulent production. The gradients over-predicted the inflow and wake region where significantly reduced turbulent energy was present. Within the jet, similar to the turbulent eddy viscosity, the under-prediction of dissipation was a result of increased turbulent energy contained in eddies smaller than the PIV resolution. The analysis also yielded the Kolmogorov length scale of the flow, which exponentially increased more slowly in high turbulence intensity inflow.

These results were used to extract the $k-\epsilon$ closure term $C_{m u}$. In the nearfield, $k \gg \varepsilon$ due to the significant turbulent energy created by the Kelvin-Helmholtz instabilities. $C_{\mu}$ was an order of magnitude smaller than the accepted value of $C_{\mu}=0.09$ [78] within this region. As the shear layer expanded and the unresolved contributions grew, $C_{\mu}$ converged within the range of $0.05<C_{\mu}<0.15$. These were commonly accepted ranges for turbulent flows, which confirms that the linear viscosity model and Modified Structure Function Method have extracted reasonable sub-grid approximations.

\subsection{Critical Point Identification and Classification}

Investigations of the coherent structures and critical points within the flow have been used to quantify changes observed in previous sections. Beginning with the $x-y$ plane, critical point analyses were performed and results along the leading edge shear layer and lee-side wake region were isolated. The $x-z$ planes were then evaluated within the lee-side wake region and around the edge of the jet.

From the method outlined in Chapter 2.5, the topological degree, D, was computed for each snapshot. A null was identified when $D \neq 0$. To account for the cancellation of two equal and opposite nulls, a triangle with a solid angle area of 
$A_{i}=0$ was divided in half and reevaluated. This process was repeated a total of three times, with all $D \neq 0$ evaluated through bilinear interpolation (from Gjøystdal [130]) to determine the presence of a critical point which was found at the spatial location of a critical point, $\boldsymbol{x}_{c p}=\left(x_{c p}, y_{c p}\right)$. Additional processing was required to determine extraneous solutions outside the current interrogation area.

Critical points identified were first classified by their $Q$-criterion $((2.68))$. For $Q>0$, a vortex was determined to be present and a border was defined surrounding all positive values. The instantaneous vorticity field of the surrounding area was defined as $\omega=\partial_{x} v-\partial_{y} u$, and the circulation was evaluated along the boundary as

$$
\Gamma=\oint \omega \mathrm{d} s
$$

All critical points were further classified through transformation to $P Q R$ space and sorted through the methods outlined in Chapter 2.5.1.

\subsubsection{Centerplane $(x-y)$}

The classified critical points on the $x-y$ plane are presented in Figure 4.42. It was assumed that two regions would to contain a significant number of critical points: a) the leading edge shear layer due to the shear layer vortices; and b) the wake vortices and unstable focus in the near wake that extended downstream. Regardless of classification, these regions were heavily populated with nulls. Along the leading edge of the jet, significantly more saddles (Figure $4.42 \mathrm{~b}, \mathrm{f}$ ) and foci (Figure 4.42c, g) were present than nodes (Figure 4.42a, e), but no differences could be determined between low and high turbulence intensity. Within the wake region, critical 
points were primarily absent in the recirculation region, but heavily concentrated immediately outside.

Additional classification through stability did not provide any indication of unique behavior between cases (statistics on flow orientation, Figure $4.42 \mathrm{~d}$ and $\mathrm{h}$ ). Thus, the critical points were further investigated through separation by location, up- or downstream of the jet centerline. The leading edge critical points were examined first. Utilizing the axes rotations defined in Freedland et al. [154] (Appendix B), the centerline was defined for each case. The trajectory locations were noted at increments of $\Delta \xi=0.1 D$. At each increment, the slope of the centerline was used to define two orthogonal lines at $\pm \Delta \xi_{*}=0.05$, which extended from the centerline to the edge of the window (see Figure 4.44a). The normal distance off the centerline, $r_{c p}$, was defined for all vortices upstream of the centerline.

Next, the average normal distance, $\bar{r}_{c p}$, was determined from a statistical analysis of all critical points within the domain (Figure 4.44b and c). The mean was then superimposed on the leading edge JCB, as defined in Chapter 4.1.2. Using this method, it was noted that the average location of vortex formation aligned well with the previous definition of the JCB (Figure 4.44a and d). In these analysis, the shear layer vortices were assumed to only consist of stable critical points, but did not yield notable statistical variations. Additional considerations will need to be made on the surrounding fluid using instantaneous snapshots.

Within the center-plane wake region, the presence of the unstable focus, defined on the $p-q$ domain as $q>4 p^{2}$ and $p<0$, repelled entrained fluid into the recirculation region or downstream into the wake. In Figure 4.44, the identification of all unstable foci confirmed the presence of these flow features, but was unable to 


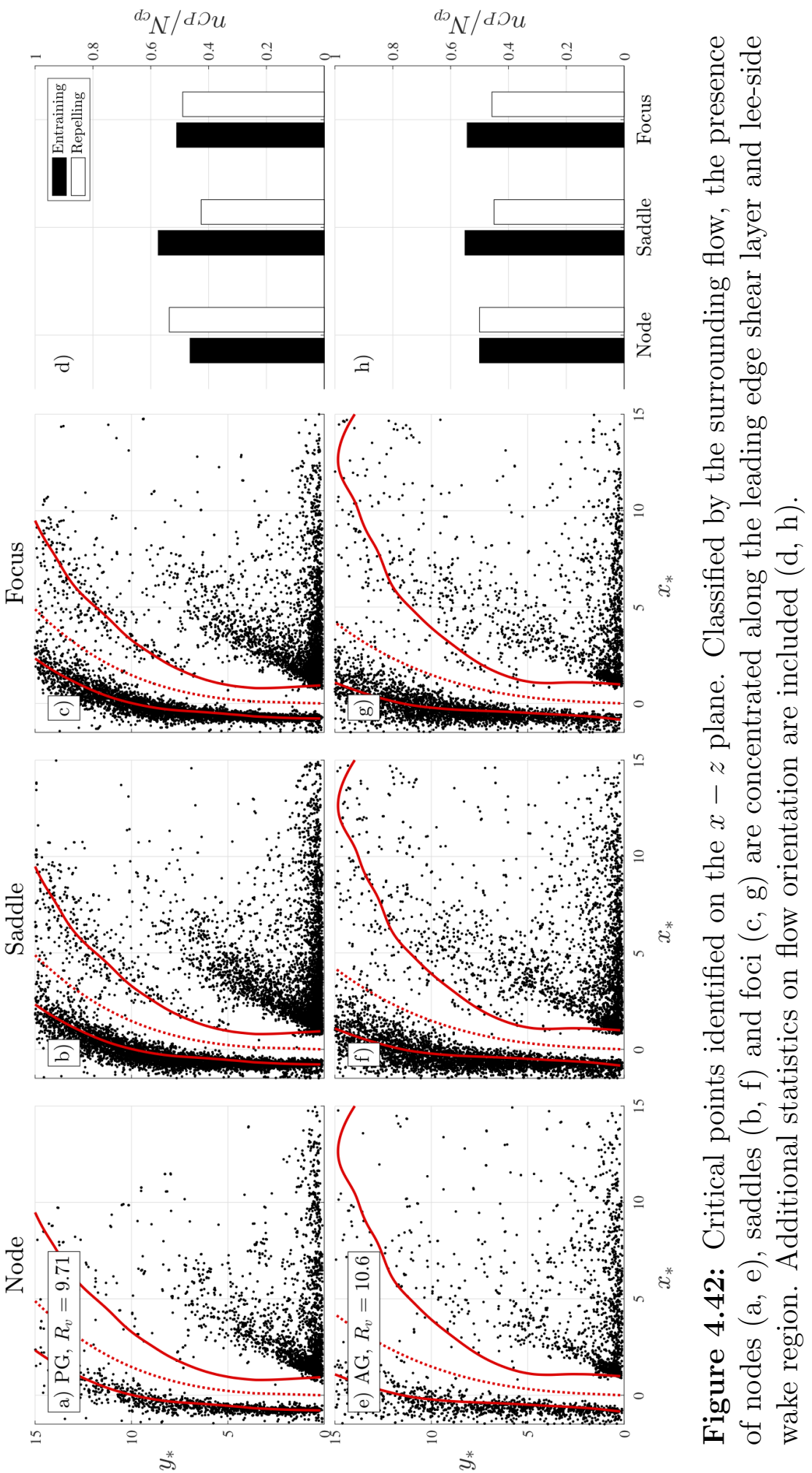



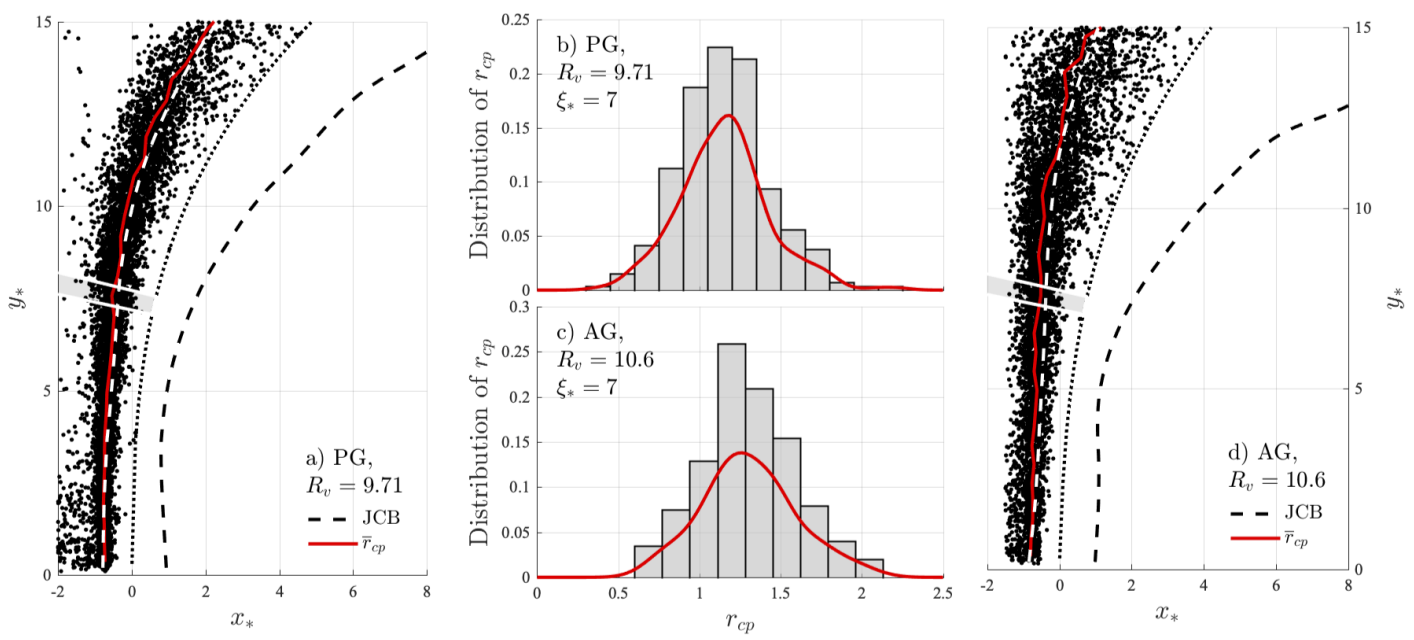

Figure 4.43: Methods of identification of leading edge shear layer critical points. At each segment of jet length, critical points were sorted and the distance off the centerline, $r_{c p}$ was determined $(\mathrm{a}, \mathrm{d})$. The distributions of $r_{c p}$ were also calculated (b, c). The mean critical point distance, $\bar{r}_{c p}$, was plotted (a, d), aligning with the leading edge JCB.

provide an exact location. Additional analysis on the mean flow fields was unable to locate any critical points. Similar to the shear layer vortices, the contributions and extent of these features will require additional analyses of instantaneous structures near the exit of the jet.

\subsubsection{Wake Vortices}

The same method was applied to each $x-z$ plane over all snapshots to provide a representative sample set. Figure 4.45 summarizes the results for the lowest crossflow velocity. Independent of elevation, the critical points were focused around and within the JCB and downstream within the wake. For the low cross-flow velocity case with skewed wake behavior (from Figure 4.17), the critical points were spread evenly throughout the wake near the floor (Figure 4.45a) but were constrained and 

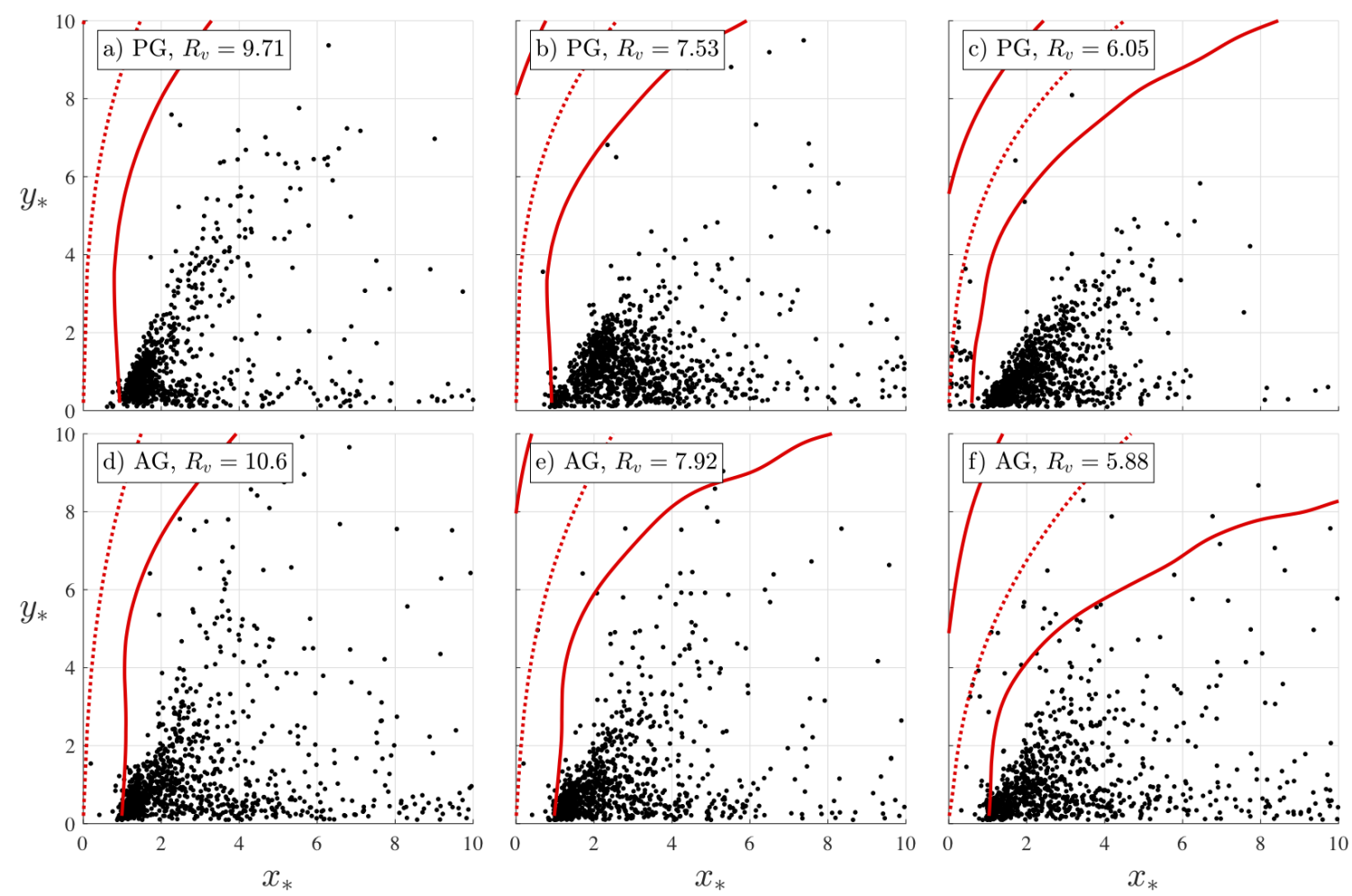

Figure 4.44: Critical points classified as "unstable foci" within the near-field wake of the jet for three PG and three AG cases.

extended in the direction of jet deformations with increasing elevation. Moving away from the floor, concentrations of critical points formed at the leading edge, which extended around the jet by $y_{*}=6$. These features were identified as the shear layer vortices, with the turbulent jet engulfing cross-flow as the shear layer formed.

Within the wake, where a prominent vortex system was present, the critical points were classified through projection onto the $p-q$ domain (from section 2.5.1). The resulting projection revealed a large quantity of critical point pairs, most notably entraining nodes with repelling critical points in close proximity. An example of this relationship is shown in the left panel of Figure 4.46. Critical point pairs were part of the same vortex and influenced the surrounding flow in tandem. Therefore, 

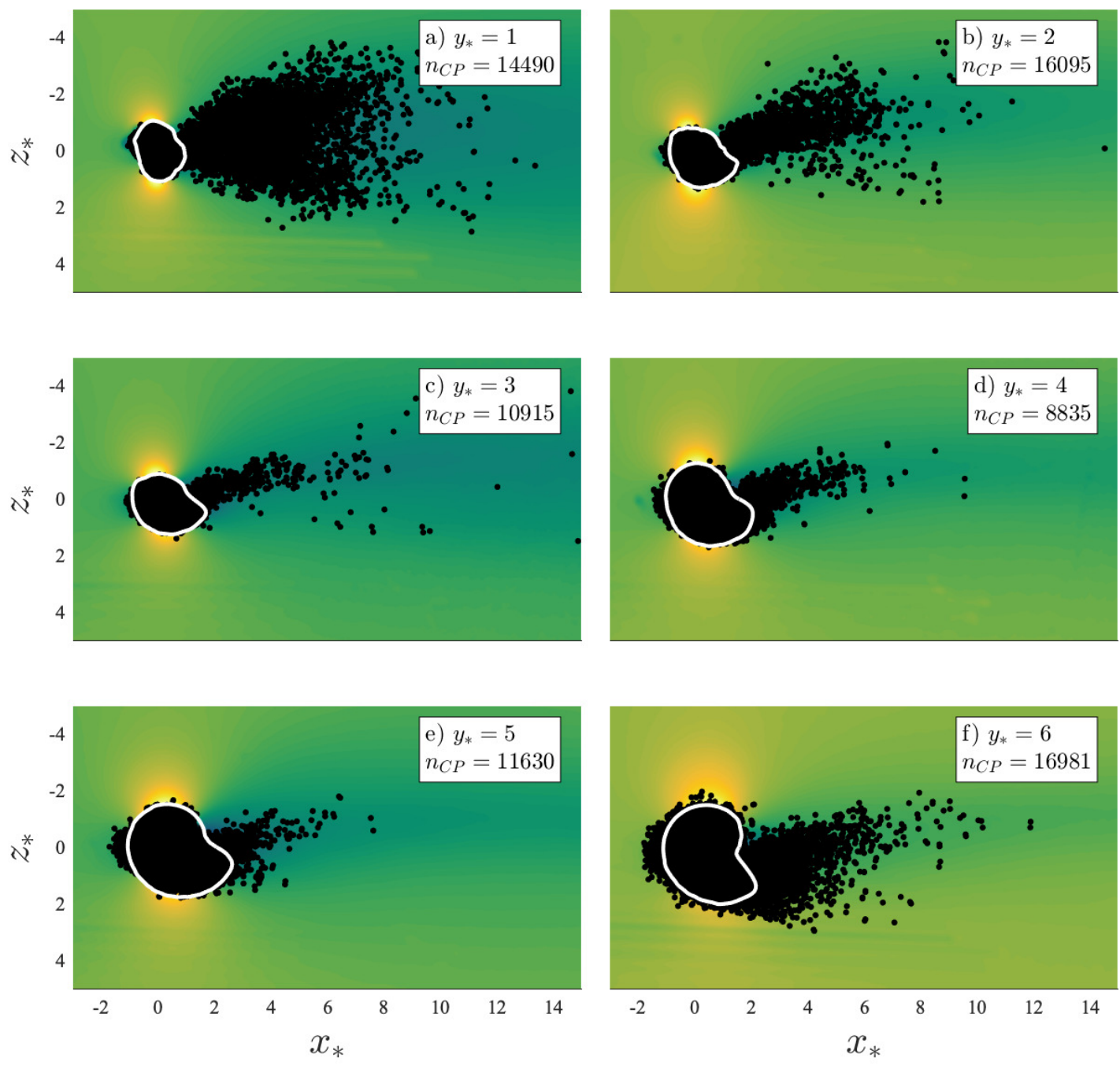

Figure 4.45: Critical point locations for $R_{v}=14$ at each elevation. The jet JCB (white) was included for spatial reference. 

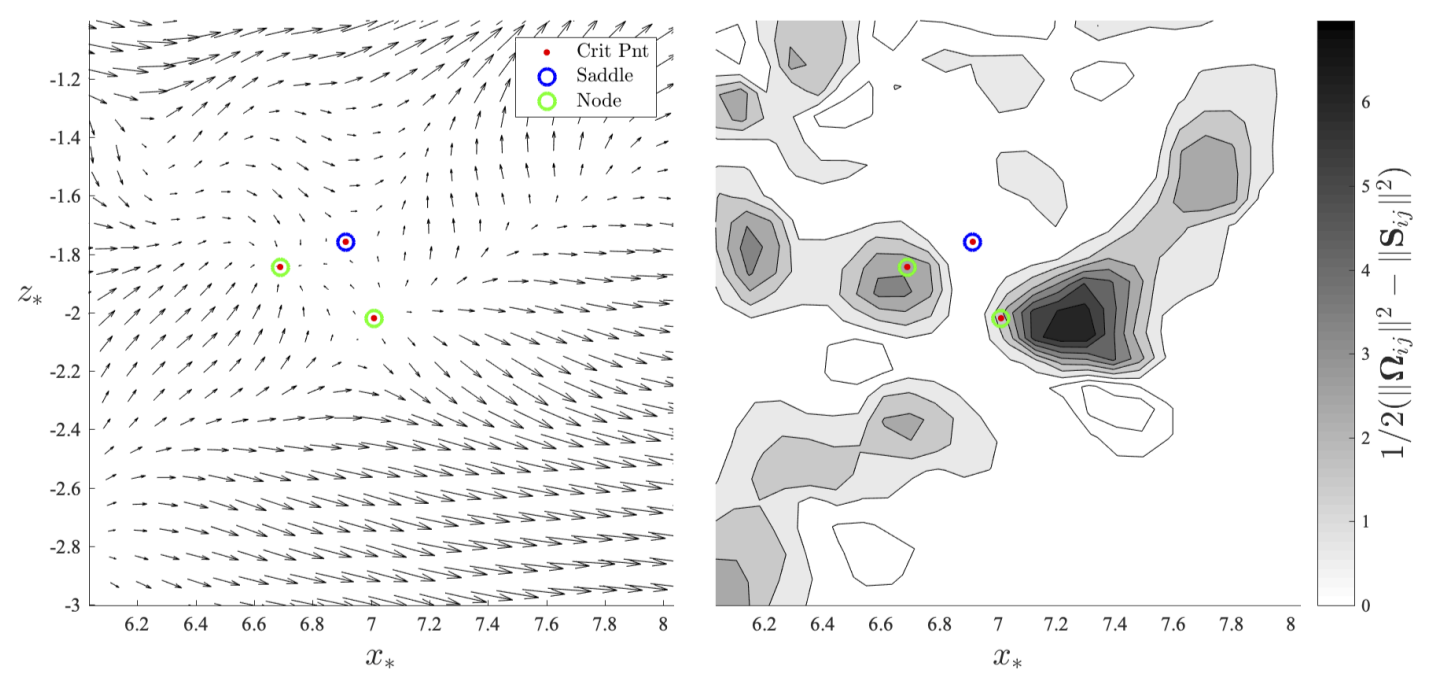

Figure 4.46: The instantaneous velocity field (left) and $Q$-criterion (right) within the wake of the jet. Critical points (red) were classified by the surrounding flow. For the pictured set of critical points, a repelling saddle (blue) and repelling node (green) surround an entraining node.

each pair was grouped to measure the size of each vortex.

The evaluation of the size of the vortices was performed through the second invariant, or $Q$-criterion, a sample of this data has been plotted in the right panel of Figure 4.46. The large connected peaks were interpreted as the extent of the vortex, with the measurement of the area providing a surface for evaluation of the vortex circulation, $\Gamma$, and a closed volume for transport analysis. In previous reports on wake vortices, the "explosion" of vorticies containing boundary layer fluid were entrained by the lee-side of the jet. It is believed that quantification of these would further improve descriptions of entrainment through the vortex systems.

\subsubsection{Summary}

In this section, the critical points within the velocity field were identified through topological degree and Greene's Bisection Method, and classified through projection 
on the $p-q$ domain. Evaluation of the $x-y$ velocity fields clarified the presence of the shear layer vortices and unstable foci in the near wake. Similarly, evaluation on the $x-z$ plane delineated the wake vortices and jet boundary.

These features provided an instantaneous location with which to assess their influence on the surrounding fluid. Interpretation of the $Q$-criterion quantified the extent of vorticies by identifying adjacent positive peaks. These then defined a boundary in which to measure the vortex circulation and transport, and will be recruited to more fully describe turbulent contributions to entrainment for JICF. This will be discussed further in chapter 6 .

\subsection{Autocorrelation of Coherent Structures}

This section focuses on the comparison of coherent structures within the flow. Twopoint auto-correlation functions (from section 2.7) were applied to the fluctuating velocity field on the $x-y$ and $x-z$ plane to measure coherent structure size and compare features across cases.

\subsubsection{Coherent Structure Measurements}

At an instantaneous momentum, the T/NT interface was deformed by the large eddies that engulfed cross-flow and entrained through viscous nibbling [2]. The smallest scales within the flow had been quantified previously in Section 4.4.3. Therefore, a quantification of the largest features within the flow was desired to describe the length scales of the energy cascade. On the $x-y$ plane, the influence of turbulent cross-flow on the shear layer vortex size was examined. The spatial correlation coefficient, $R_{i j}$, from (2.75) was evaluated along the leading edge JCB. The angle 

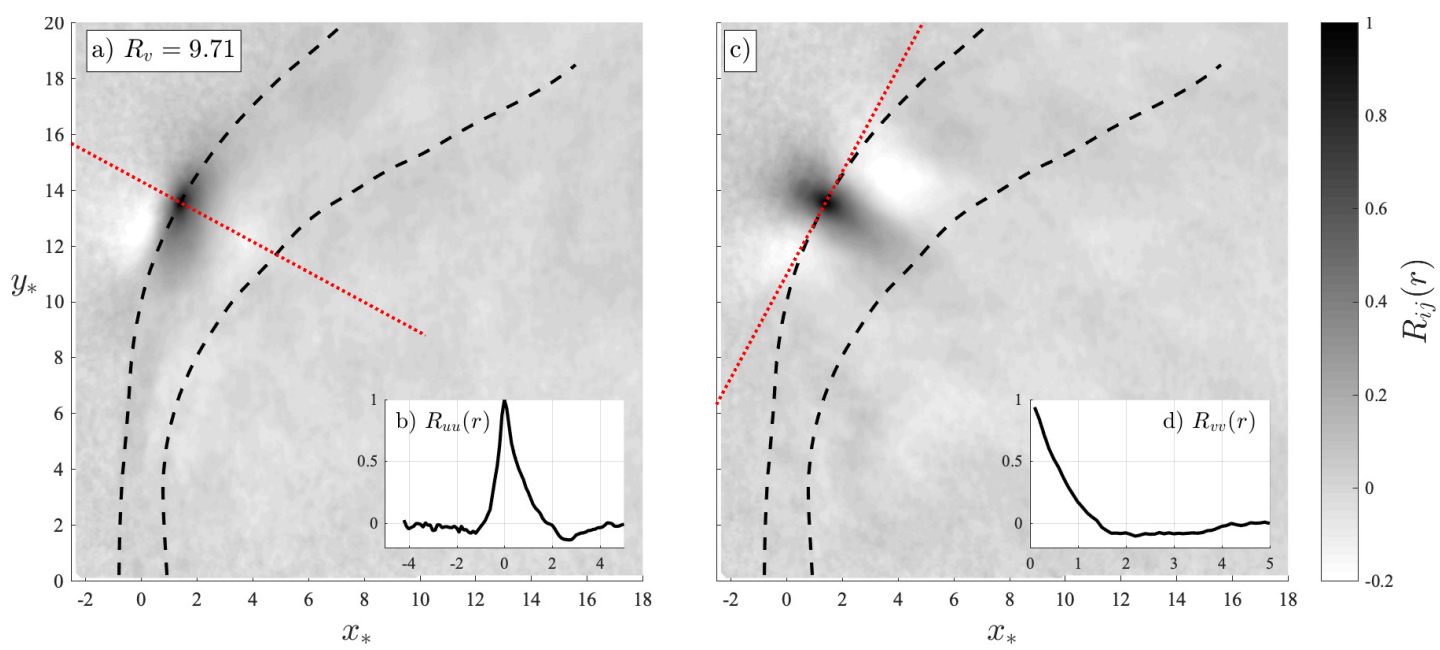

Figure 4.47: Two-point correlation fields evaluating equation (2.75) along the leading edge JCB (dashed) for a) $\phi_{i}=\psi_{j}=u_{r}^{\prime}$ and c) $\phi_{i}=\psi_{j}=v_{r}^{\prime}$. Autocorrelation functions were interpolated along the dashed red lines. The resulting auto-correlation curves $R_{u u}(r)$ (b) and $R_{v v}(r)(\mathrm{d})$ were integrated to yield the length scale of the coherent structures.

relative to the cross-flow, $\theta_{L E}$, was determined and the velocity components were transformed using the procedures outlined in Appendix B. Reorientation of the flow allowed measurement of the eddying motions in the direction of the flow. Two evaluations were then made. First, to measure the penetration of the coherent structures upstream and into the shear layer, the fluctuating components were defined normal to the JCB, where $\phi_{i}=\psi_{j}=u_{r}^{\prime}$. Then, to identify coherent structures moving along the leading edge JCB, the tangential fluctuating components were evaluated as $\phi_{i}=\psi_{j}=v_{r}^{\prime}$. An example is presented in Figure 4.47a and c.

For $R_{u u}(r)$ (Figure 4.47b), the autocorrelation functions were evaluated in both directions to measure the eddy penetration into the cross-flow $(r<0)$ and into the jet $(r>0) . \quad R_{v v}(r)$ (Figure $\left.4.47 \mathrm{~d}\right)$ was evaluated in the direction of fluid motion $(r>0)$. The integral length scale, defined as $L=\int R_{i j}(r) d r$, was evaluated from 
the peak to the first zero crossing for consistency throughout measurements. The measurement of the shear layer vortices in two dimensions provided two descriptions of the flow: a) the "reach" of coherent structures outside the shear layer and into the jet $\left(R_{u u}(r)\right)$, and $\left.\mathrm{b}\right)$ the length scale of the developing vortices moving along the $\operatorname{JCB}\left(R_{v v}(r)\right)$.

The reach of coherent structures described the distance off the JCB that a shear layer vortex could engulf cross-flow upstream. When cross-flow was engulfed by the largest eddies along the JCB, it became turbulent through viscous nibbling and was imparted with turbulent energy. This energy was transferred to smaller scales through the energy cascade. Accordingly, an evaluation of the reach of turbulent structure was sought to provide another description of the shear layer vortices. The length scale was determined at each $\xi_{*}$, with the normal directed out into the crossflow and $R_{u u}(r)$ was integrated from the peak to the first zero crossing. The results for select cases were presented in Figure 4.48.

Similar to previous results, the role of cross-flow turbulence was minimal in the near-field. Before the collapse of the core, the PG and AG cases increased linearly and cross-flow turbulence increased the rate of growth. At the collapse of the core, the coherent structures were $\sim 20 \%$ larger for AG. For $\xi / \xi^{0}>1$, the PG cases continued to linearly increase in size. Similar to the JCB definitions from section 4.1.2, the AG was dramatically impacted by the increased inflow turbulence. The coherent structures exponentially increased through the remainder of the jet. Therefore, the eddies present along the leading edge of the jet were able to engulf cross-flow much farther upstream than the PG case, significantly increasing the shear layer expansion. 


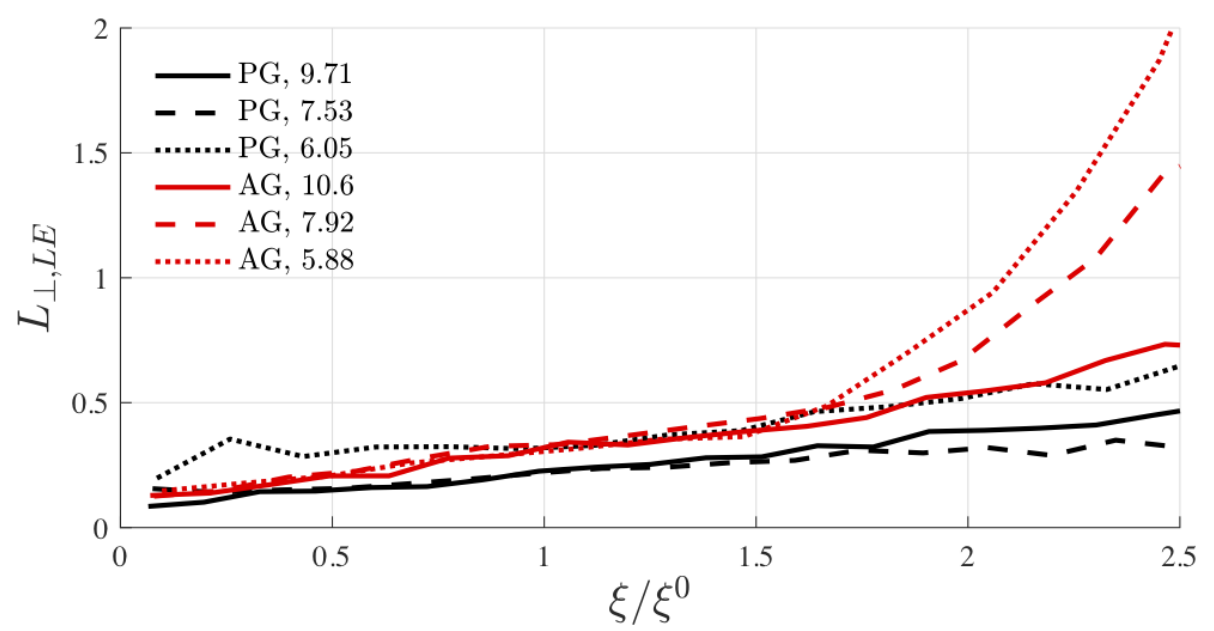

Figure 4.48: The length scale of coherent structures evaluated at the leading edge JCB with the normal displacement vector directed outward. The radial autocorrelation function, $R_{u u}(r)$ (from Figure 4.47b), was integrated from the peak to the first zero crossing.
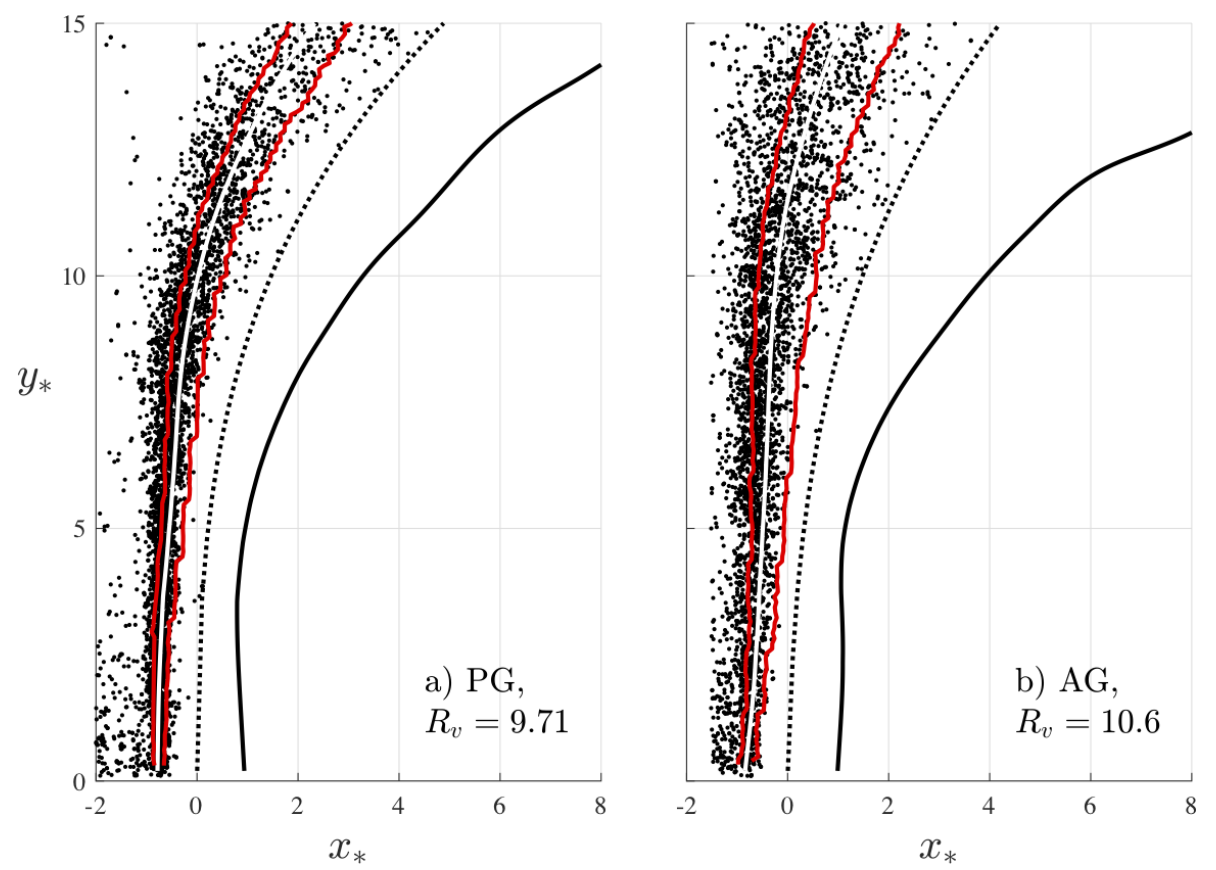

Figure 4.49: The integral length scale evaluated at the JCB with the displacement vector directed upstream and into the jet. The radial autocorrelation function, $R_{u u}(r)$ (from Figure 4.47), was integrated from the peak to the first zero crossing. 
These lengths will provide additional considerations for future analysis. In section 4.5.1, the large quantity of critical points complicated calculations of summary statistics. Projecting the length scales off the JCB, the regions of correlated features provided a reduced region for classification of critical points (Figure 4.49). A combination of the measured length of coherent structures and instantaneous features of the flow reduced the complexity, providing significant filtering of extraneous features.

\subsubsection{Comparison of Coherent Structures}

Use of the two-point correlation function, $R_{i j}$, helped to identify the extent of coherent structures within the flow-field, but was also useful in comparing features across varying flows. For example, contrasting the turbulent features in the jet for varying cross-flow velocity was accomplished by evaluating similar features at varying locations.

On the $x-y$ plane, three regions were investigated (Figure 4.50) and points were chosen based on measured features from previous sections and prior reports from the literature. These were, first, within the jet, the peak production of turbulence (from Figure 4.14); second, the collapse of the potential core (from Figure 4.6); and third, the far-field transition point (as defined by Smith \& Mungal [31]). For low inflow cases, an additional location within the fully turbulent jet enhanced the analyses and was defined at $\xi=2 \xi^{0}$. On the lee-side of the jet in the near-wake, the recirculation region was defined at the center. In the wake moving downstream, three locations were chosen to directly compare development of the wake region at $x_{*}=5,10$, and 15. All velocity components were transformed and evaluated 

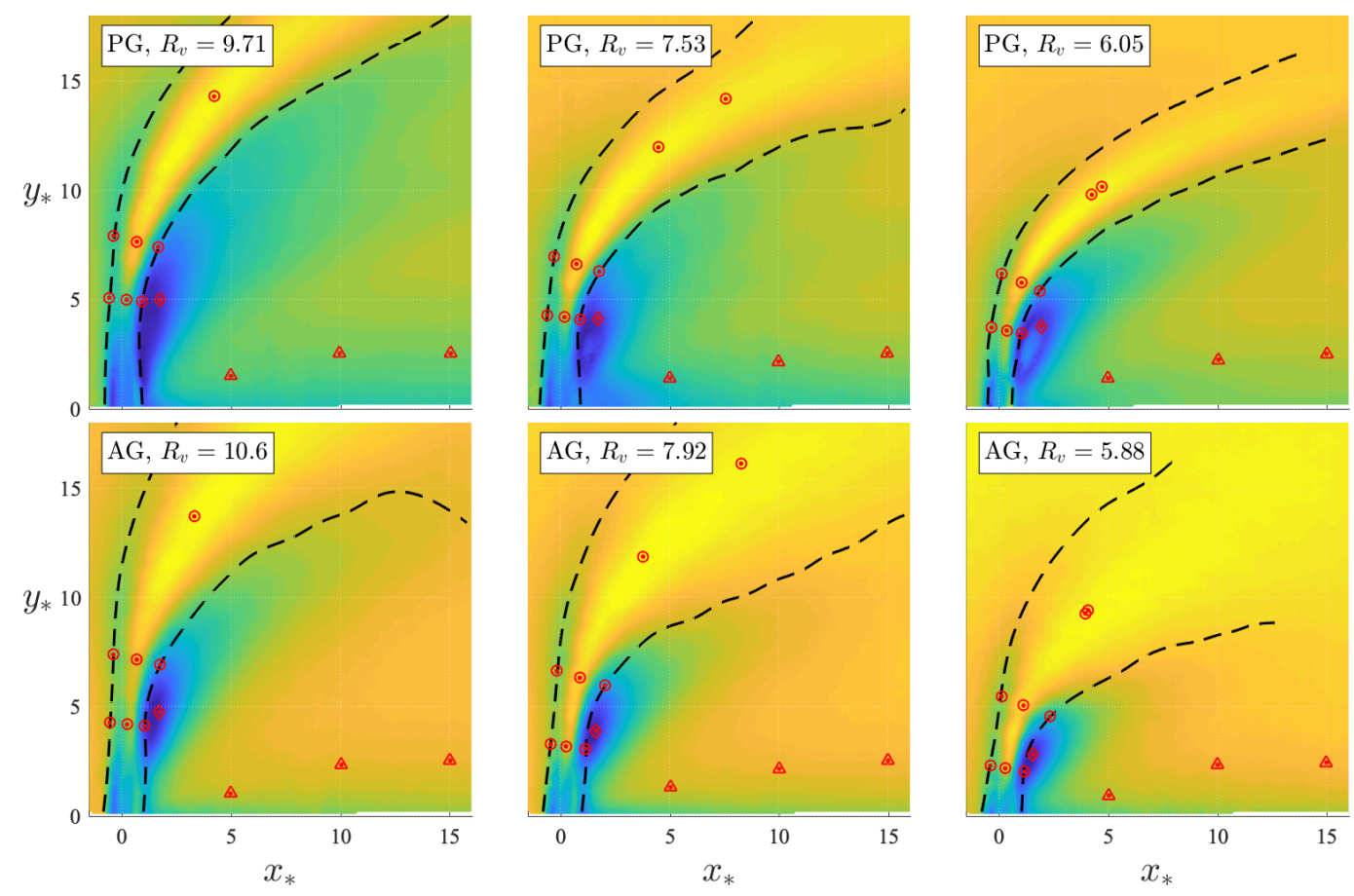

Figure 4.50: Locations of similar features across cases located within the jet $(\circ)$, the near-wake recirculation region $(\diamond)$, and the downstream wake $(\triangle)$.

tangential to the magnitude of velocity $\left(R_{u u, r}\right)$ similar to the results in section 4.4 (see Figure 4.35). The resulting correlation maps were compared across similar crossflow velocities. Three interesting results will be reviewed in the context of variations in wake behavior: the streamwise fluctuations within the wake (at $x_{*}=10$ ), in the center of the near-wake recirculation region and within the jet (at $\left.\xi^{0}\right)$.

Within the wake region, the role of turbulent cross-flow had been identified through transport. It was observed that the wake recovery increased for the AG cases. This has been previously observed in the published literature (see [147, 148, 149]). Evaluating $R_{u u, r}$ at $x_{*}=10$ (Figure $4.51 \mathrm{a}, \mathrm{b}, \mathrm{c}$ ), a location where the wake had recovered for AG but not for PG, complimentary correlations reconfirmed this observation. For PG, the coherent structures were uncorrelated $1 D$ up and 

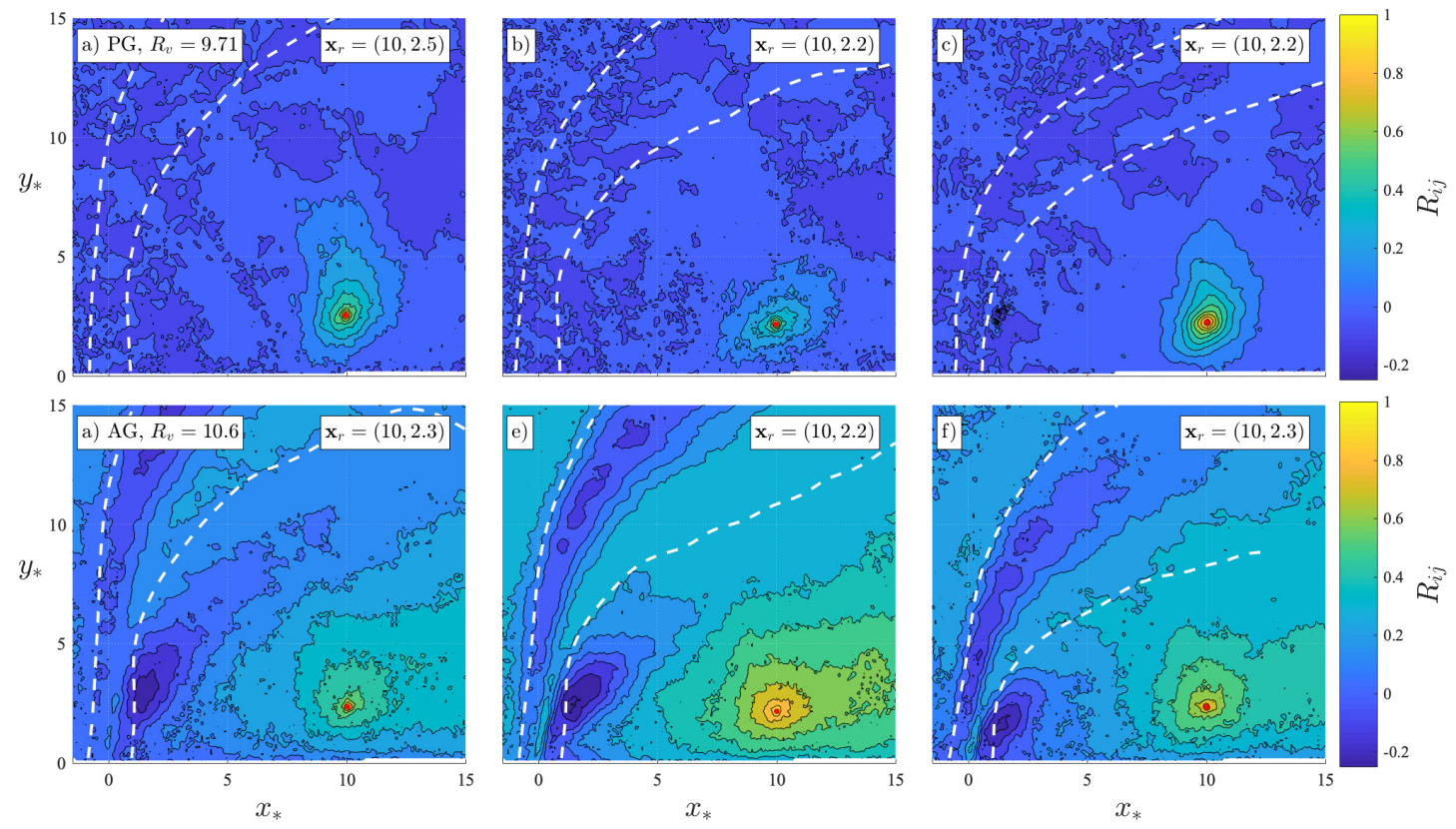

Figure 4.51: Auto-correlation of coherent structures, $R_{u u, r}$, for the streamwise fluctuating field within the wake at $x_{*}=10$ for three PG and three AG cases.

downstream and to the inflow, an indication the wake had not recovered and the features present were unique in the flow.

For the AG cases, the significant increase of correlated features reinforced the idea that the wake had significantly recovered by $x_{*}=10$ (Figure $4.51 \mathrm{~d}$, e, f). The reasonable correlations $\left(R_{u u, r}>0.35\right)$ extending several diameters up- and downstream of the jet and in the inflow indicated that the coherent structures were different, but behaved similarly to other features in the flow. Interestingly, the nearwake recirculation region yielded a negative correlation coefficient. Therefore, the vortex shedding in the near-wake did not correlate downstream and was shown to dissipate quickly as the wake returned closer to inflow conditions.

This finding reinforced previous observations from the transport, specifically 
those related to Figure 4.16. The large differences to the inflow for PG transport described significantly different features in the wake that persisted downstream. The small increase and quicker return of Reynolds stress contributions to inflow conditions for AG indicated the coherent structures would be similar in the wake for AG.
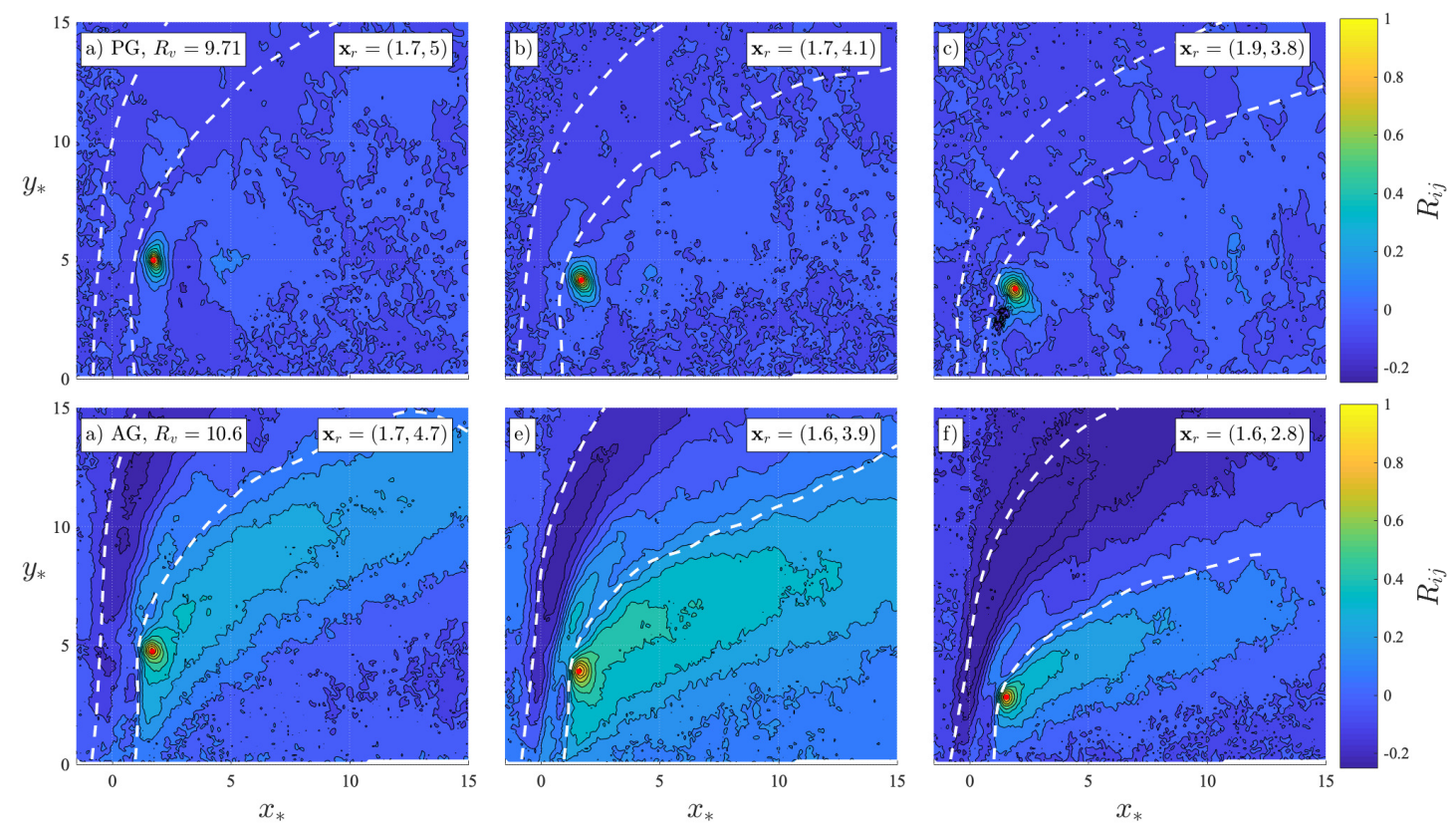

Figure 4.52: Auto-correlation of coherent structures, $R_{u u, r}$, for the streamwise fluctuating field within the near-wake recirculation region for three PG and three AG cases.

The near-wake region was then investigated to identify the negative correlations observed. In Figure 4.52, $R_{u u, \perp}$ was evaluated at the center of the near-wake recirculation region. Similar to the wake, the near-wake behavior varied significantly for low and high inflow turbulence. For PG (Figure 4.52a-c), low coherence indicated the secondary flow evolved over the length of the jet. The formation of the counter- 

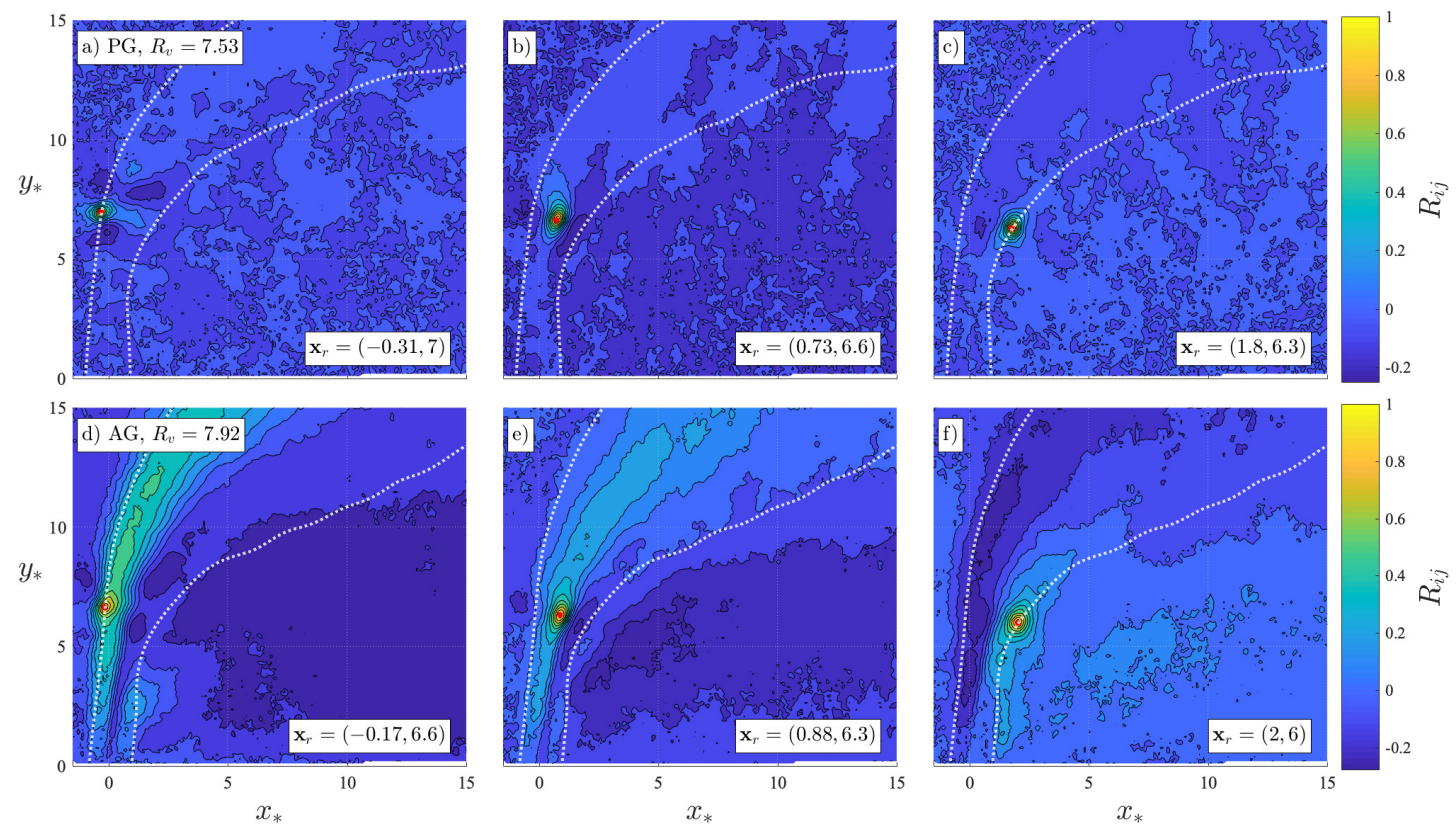

Figure 4.53: Two-point correlation, $R_{u u, r}$, evaluated at the leading edge JCB (a, d), centerline (b, e) and lee-side JCB (c, f) at $\xi^{0}$ for one PG and one AG case.

rotating vortices entrained cross-flow on the lee-side of the jet and the location it entrained had significant impact on the coherent structures present. For AG, strong coherence extended along the entire near-wake and within the JCB of the jet. Thus, the entrained cross-flow, which already contained turbulent fluctuations, created a more uniform flow on the lee-side. The negative coherence to the leading edge of the jet indicated the entrained cross-flow on the lee-side did not mix within the jet.

This was confirmed evaluating the $R_{u u, r}$ within the jet at three sites: the leading edge, centerline, and lee-side at $\xi=\xi^{0}$ (Figure 4.53). The inflow turbulence was found to have a dramatic influence on the coherence of turbulence within the jet. For PG (Figure 4.53a-c), all coherence dissipated close to $\mathbf{x}_{r}$, indicating the turbulent 
features at the collapse of the core were unique. The emerging vortex systems, which continuously developed until the return of self-similarity, impacted the turbulent behavior and removed all similarity in the flow.

For the AG case, which was previously found to contain far more small scale contributions, strong coherence extended along the jet length. At the leading edge (Figure 4.53d), where shear layer vortices were observed, $R_{u u, r}>0.5$ was recorded several diameters up and downstream. The centerline exhibited similar behavior (Figure 4.53e), but a reduction of $\sim 20 \%$ coherence was observed. While the turbulent features were less similar, the extended coherence reinforced the persistence of sub-grid contributions recorded in section 4.4.1. Interestingly, the lee-side of the jet negatively correlated to both the leading edge and centerline. The opposite was observed for $R_{u u, r}$ at the lee-side JCB (Figure 4.53f).

The coherence along the JCB that extended into the wake and the negative correlations along the leading edge confirms that the two regions were influenced by different turbulent features. The leading edge, which continuously interacted with and entrained cross-flow, did not dissipate as additional turbulent energy was added to the system. And the entrainment of cross-flow through shear layer vortices was unable to alter the coherent structures significantly until after self-similarity returns. On the lee-side, coherence between the recirculation region and lee-side of the jet was observed (similar to the results in Figure 4.51). The turbulent cross-flow entrained by the jet in the near-wake was similar to the turbulence further downstream in the wake. This similarity in the wake, coupled with the absence of out-of-plane velocity, could be attributed to the dissipation of the counter-rotating vortices. The vortices mixed the entrained flow within the jet, removing all coherence with other regions of 
the flow (Figure 4.53a-c). In addition, this would also explain the rapid expansion of the shear layer into the wake region, as the jet was not bound by the self-contained vortex system and could expand as a free-shear flow downstream into the wake.

\subsubsection{Summary}

In this section, descriptions of turbulent features through autocorrelation functions confirmed observations from previous sections and improved upon our understanding of jet and wake behavior. Definition along the JCB allowed the measurement of the size of the coherent structures present. Integration of $R_{i j}(r)$ normal to the JCB defined the reach of the shear layer vortices into the cross-flow and the distance within the jet that would be present. Similar to the expansion of the shear layer, the role of turbulent inflow dramatically increased the reach upstream of coherent structures, an indication of increased engulfment.

The evaluation of the wake region also confirmed the increased wake recovery for AG cases. The strong increase in correlated features within wake and negative correlation to the near-wake confirmed that the turbulent features returned closer to inflow conditions by $x_{*}=10$. This result had been observed previously through momentum and energy transport analyses.

The coherent structures present in the near-wake recirculation region did not correlate outside the immediate vicinity for $\mathrm{PG}$, indicative of changing turbulent features. The stronger coherence that was present for AG confirmed that the entrained flow was not altered significantly by the jet. Investigation within the jet identified two regions of coherence: the leading edge to the centerline, and the leeside of the jet. The two anti-correlated regions suggested two distinct turbulent 
features that did not mix. A clear reduction in turbulent mixing could be explained by the dissipation of the counter-rotating vortices, which circulated entrained flow in two self-contained coherent structures. The jet would then expand as a free-shear flow and turbulent features would persist further downstream.

\subsection{Low Order Model Investigation}

In previous sections, the turbulent features within the flow had been calculated directly or summarized through bulk terms. Once complete, these calculations could accurately describe the general behavior of the flow. However, analyses such as these are time intensive and in the event of a catastrophic natural occurrence such as a volcanic eruption, would be too slow to create accurate predictive models that could effectively warn nearby populations. This section describes the development of the

jet, which was optimized for the reduced order model, Plumeria [8], to remove all turbulent features from the flow. Evaluation of the system could then occur as a one dimensional mass balance through the simplified closure terms, $\alpha$ and $\beta$.

From McNeal et al. [157], optimized entrainment coefficients were extracted by evaluating the convergence of the modelled centerline to the experimental data. But this situation only accounted for one output and was not able to incorporate the shear layer growth. Defining initial conditions and the two closure terms, Plumeria generated spatial locations along the centerline $(x, y)$ and a radius, $r$, at each location. These results were then compared to the centerline and shear layer results (in chapters 4.1.2 and 4.1.3) for three inflow velocities. Assuming axisymmetric expansion, the centerline and shear layer edges were defined and compared for a small range of $\alpha$ and $\beta$ in Figure 4.54. 

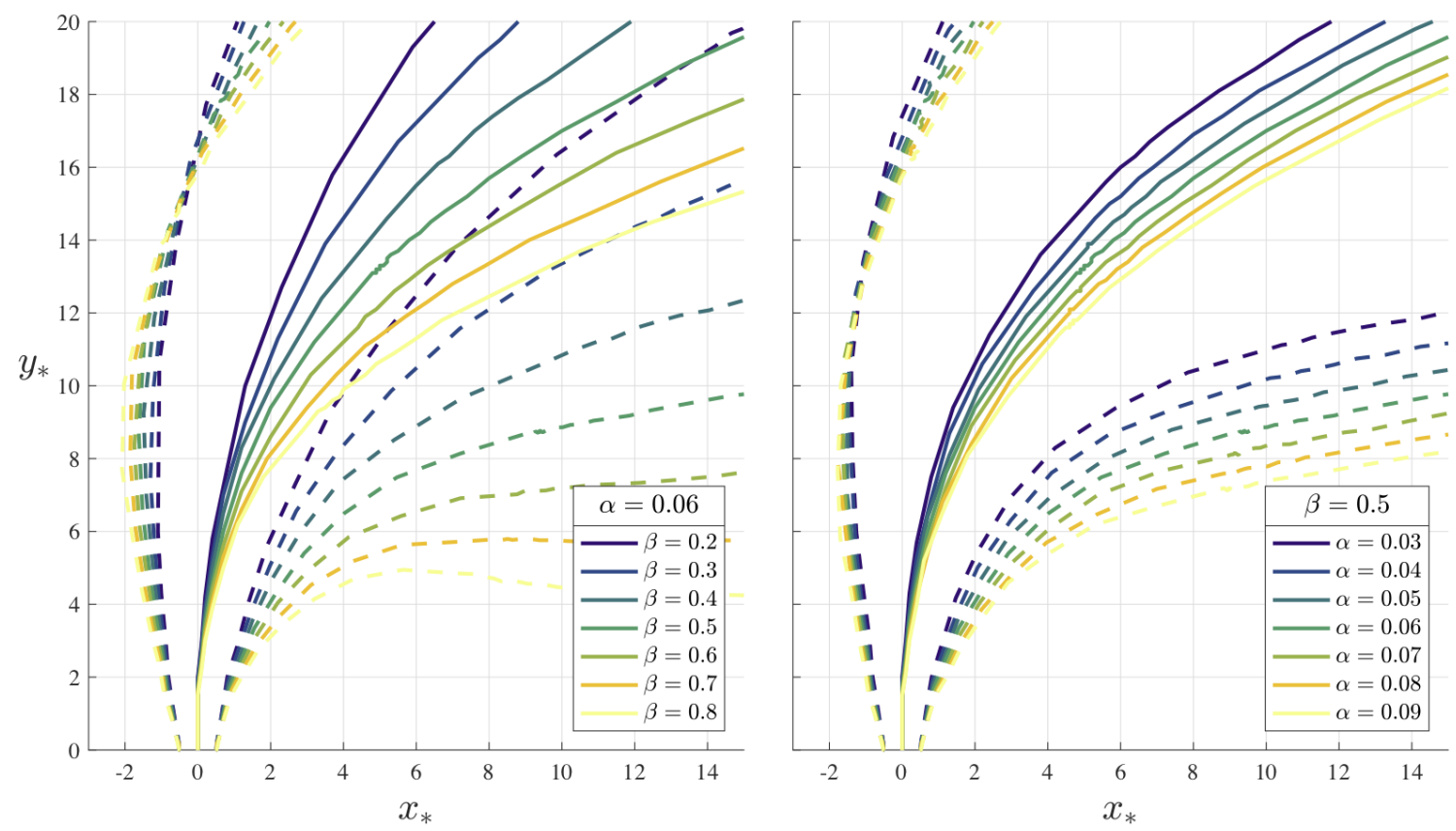

Figure 4.54: Plume centerline and shear layer output from Plumeria [8] identifying the dependence on $\alpha$ and $\beta$ for $R_{v}=14$.

The baseline investigation identified the strong dependence of $\beta$ on both the centerline and shear layer expansion for a constant $\alpha$. From the definition of the entrainment velocity (Equation (2.17)), the cross-flow contributions began after the plume began to deflect. With higher $\beta$, the cross-flow entrainment continued to increase and yielded a greater prediction of plume size. For variations in $\alpha$, the final plume shape was not as severely impacted over the full range of values. These observations provided insight into optimization of both $\alpha$ and $\beta$ terms. For the PG cases, the minimized shear layer expansion would benefit from a fixed $\beta$, while AG cases would benefit from a larger range of $\beta$.

Matching the center-lines through residual analysis, the "goodness of fit" was computed as

$$
1-\sum\left(y_{C L}-y_{P l}\right)^{2} / \sum\left(y_{C L}-\overline{y_{C L}}\right)^{2} .
$$




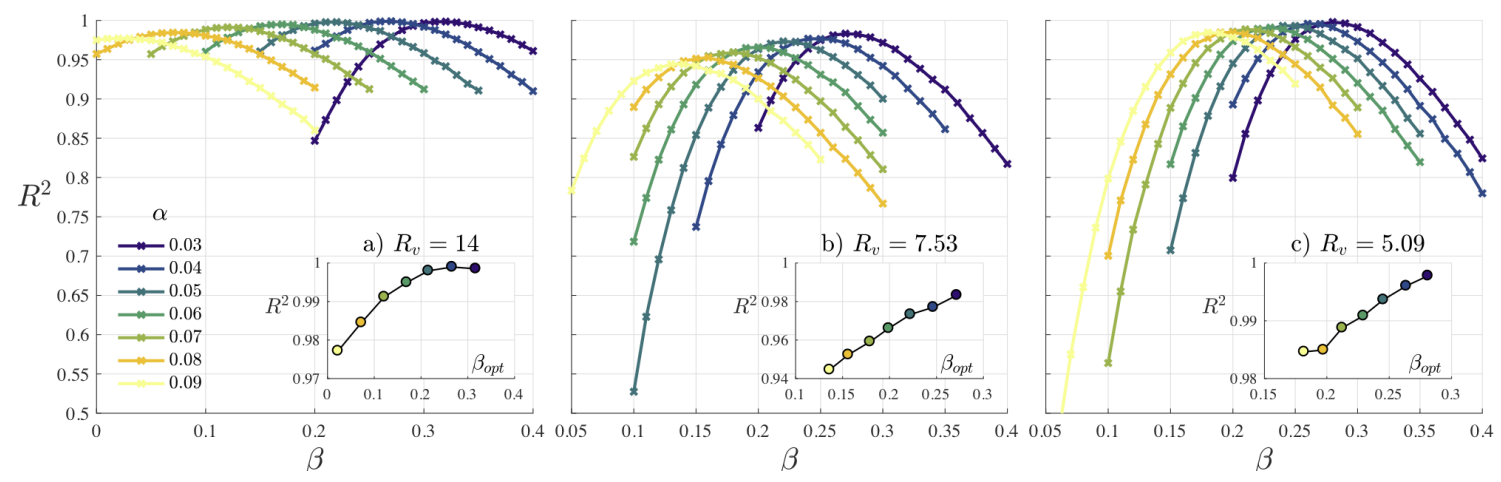

Figure 4.55: Evaluation of the fit quality through residual analysis. For a constant $\alpha$, a range of $\beta$ were compared to experimental centerline data. The best $\beta$ for each $\alpha$ was determined (inset plot).

The results can be found in Figure 4.55. After optimization of $\beta$, not all values of $\alpha$ yielded an optimal fit. In Figure 4.55a, the best $\alpha$ and $\beta$ were determined by extrapolating the peak. For increasing cross-flow velocity, the results did not allow accurate extrapolation. For $R_{v}=7.53$ (Figure 4.55b), the theoretical optimal $\alpha$ would be negative, indicating an over-prediction of cross-flow entrainment and correction with lost radial entrainment.

Keeping these results in mind, the plume shape was then compared by evaluating the two-dimensional area determined in section 4.1.3. Similar to the previous comparison, the optimal $\beta$ was determined for a range of $\alpha$ and has been presented in Figure 4.56. The extracted values from Figure 4.57 (red) and optimal centerline terms (dashed) were included for comparison. With the added complexity of shear layer expansion, the optimal $\beta$ values were nearly half the optimal centerline results. So, while the theoretical models could not match the centerline exactly, the complex near field expansion of the jet was predicted with compromises to the centerline trajectory.

Finally, the $x$-z plane jet shape was employed to optimize the near-field volume. 


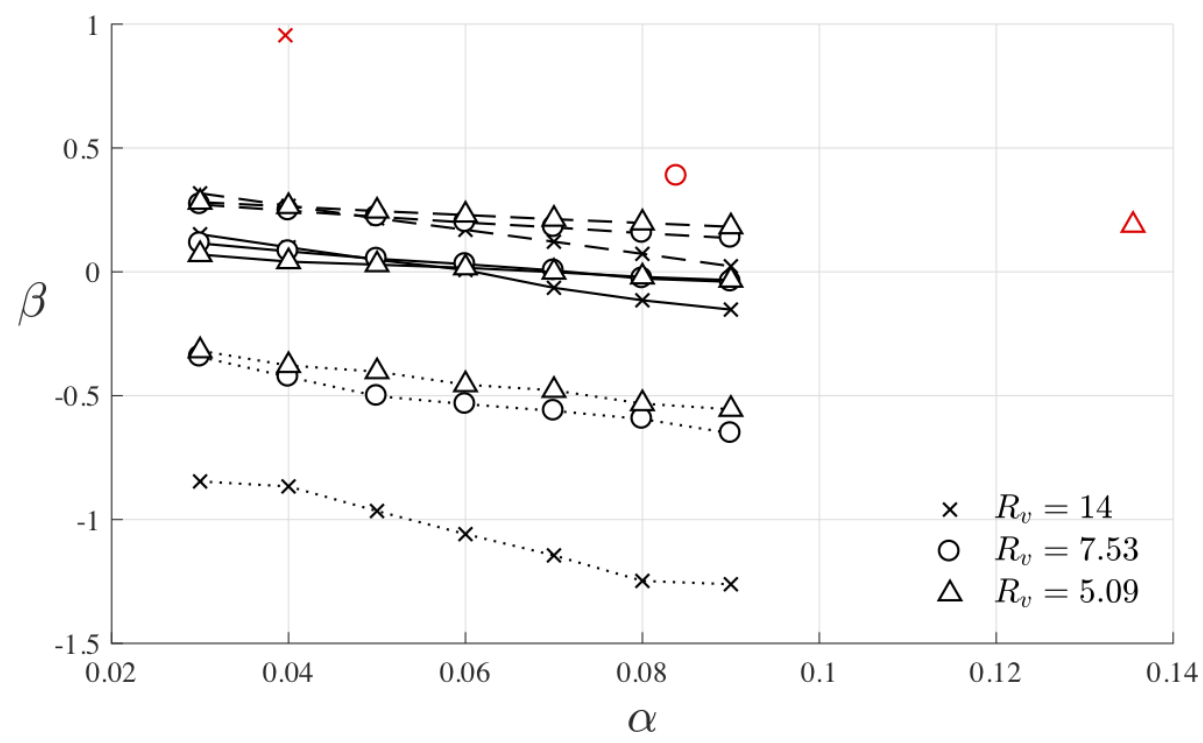

Figure 4.56: Optimal $\alpha$ and $\beta$ to predict centerline (solid), center-plane area (dashed) and near-field volume (dotted) for three inflow velocities. Computed $\alpha$ and $\beta$ from mean flow statistics (red) are included for comparison.

The Plumeria outputs were converted to conic sections at each height using the method in Appendix C. Resolved data only allowed for analysis through $y_{*}=6$, which suggests that only the complex deformations would be optimized. In Figure 4.20, no change in area was observed near the floor when $y_{*}<3$. Therefore, to achieve a reasonable solution, $\beta$ must account for these features. In Figure 4.56 (dotted lines), the optimal $\beta$ varied greatly with inflow conditions. For low crossflow velocity, optimal closure terms ranged from $-1.25<\beta<-0.8$, due to the nearly negligible jet deflection angle in the near-field. With increased inflow velocity, $\beta$ decreased to $\sim-0.5$. While generally accepted, the negative sign indicated that this method was not reliable for optimizing entrainment coefficients. 


\subsubsection{Direct Calculation of Closure Terms}

From Chapter 2.2, the entrainment coefficients were used to summarize the entrainment velocity, $u_{e}$ (defined in (2.17) as $u_{e}=\alpha\left|u_{c}-u_{\infty} \cos \theta_{C L}\right|+\beta\left|u_{\infty} \sin \theta_{C L}\right|$ ). These coefficients were directly solved from the collected velocity data. First, the mean flow statistics on the relative flow-field (from Figure 4.5) was evaluated at the edge of the JCB in Chapter 4.1.3. Secondly, the mean flow statistics on the $x-z$ plane were transformed using polar coordinates at the edge of the jet (from Figure 4.18). A system of equations, $\mathbf{A} \vec{x}=\vec{b}$ were then designed to solve for $\alpha$ and $\beta$ directly from the definition of the entrainment velocity, where

$$
A=\left[\begin{array}{cc}
a_{1}\left(\xi_{1}\right) & a_{2}\left(\xi_{1}\right) \\
a_{1}\left(\xi_{2}\right) & a_{2}\left(\xi_{2}\right) \\
\vdots & \vdots \\
a_{1}\left(\xi_{n}\right) & a_{2}\left(\xi_{n}\right)
\end{array}\right], \quad b=\left[\begin{array}{c}
u_{e}\left(\xi_{1}\right) \\
u_{e}\left(\xi_{2}\right) \\
\vdots \\
u_{e}\left(\xi_{n}\right)
\end{array}\right]
$$

and

$$
\begin{aligned}
& a_{1}(\xi)=\left|u_{C L}(\xi)-u_{\infty} \cos \theta_{C L}(\xi)\right| \\
& a_{2}(\xi)=\left|u_{\infty} \sin \theta_{C L}(\xi)\right| \\
& u_{e}(\xi)=\bar{U}_{J C B}(\xi) \cos \theta_{C L}(\xi)-\bar{V}_{J C B}(\xi) \sin \theta_{C L}(\xi) .
\end{aligned}
$$

On the $x-y$ plane, this system of equations was applied along the leading edge and lee-side JCB through the entire length of the jet to simulate constant entrainment coefficients. In Figure 4.57 ( $\mathrm{a}$ and $\mathrm{b}$ ), $\alpha$ and $\beta$ varied as cross-flow velocity and turbulence intensity were changed. For increasing cross-flow velocity, the radial 

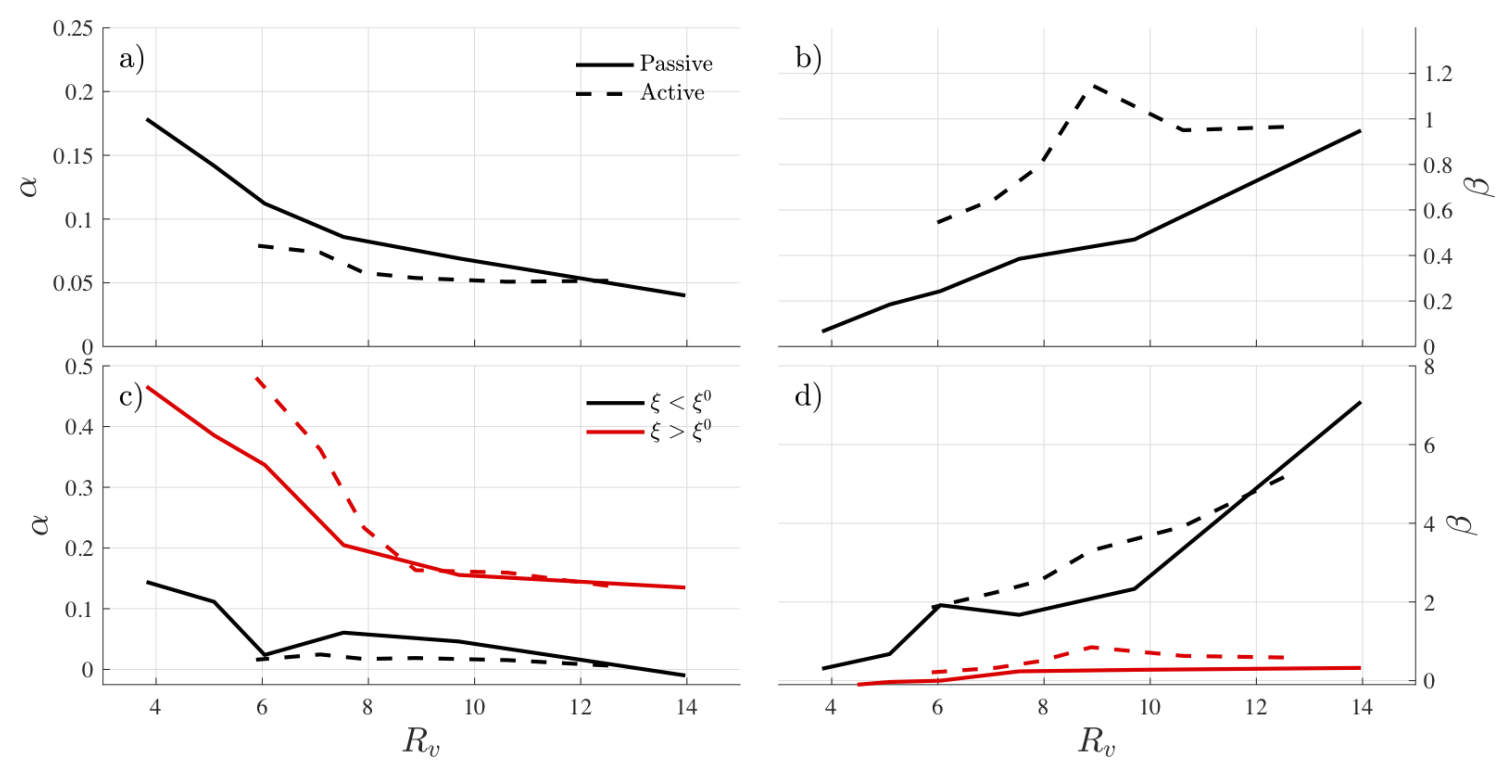

Figure 4.57: Entrainment coefficients a) $\alpha$ and b) $\beta$ for low and high inflow turbulence. The jet was separated and the near- and far-field components were reevaluated $(\mathrm{c}, \mathrm{d})$.

entrainment, $\alpha$, increased while the cross-flow entrainment, $\beta$, decreased. Both closure terms were within the range of accepted values, with $\alpha$ ranging from 0.04 to 0.18 and $\beta$ from $\sim 0.05$ to 1 . For low cross-flow turbulence, $\alpha>\beta$ when $R_{v}<5$. This was attributed to the formation of the counter-rotating vortices and secondary flow. The additional fluid near this region was entrained by the vortex systems, increasing the contributions of cross-flow entrainment.

The trends were similar for high inflow turbulence, with $\alpha$ increasing and $\beta$ decreasing for increasing cross-flow velocity. When compared to the PG results, a decrease in $\alpha$ and increase in $\beta$ was observed, with increases in $\beta$ greater than the losses in $\alpha$. This had significant impact on the expansion of the shear layer (as observed in the left panel of Figure 4.54). The large impact on the plume size indicated that the entrainment coefficients accounted for the increased growth for 
high cross-flow turbulence. Therefore, these values provided a useful range of $\alpha$ and $\beta$ to test within the Plumeria model.

The near- and far-field behavior would behave quite differently, specifically before and after $\xi^{0}$. In Figure 4.57c and d, those two regions of the flow were separated and the entrainment coefficients were evaluated. Within the near-field, where the shear layer expansion was muted and the jet deflection was nearly negligible, $\alpha$ behaved similar to the total entrainment, but was smaller in magnitude across inflow velocity and turbulence intensity. For $\beta$, an increase of nearly $800 \%$ was determined to compensate for low $\theta_{C L}$. After the collapse of the core, when shear layer expansion increased and the jet bent downstream, $\alpha$ more than doubled and $\beta$ dropped back into the range of accepted values $(\beta<1)$. These results provided additional ranges with which to test in Plumeria, with the goal of identifying a method for compensating for reduced near-field entrainment.

Identification of the near-field dynamics in terms of $\alpha$ and $\beta$ was further investigated on the $x$ - $z$ plane. Equation (4.9) was applied to each plane at the JCB and closure terms were evaluated. Due to the nearly negligible centerline deflection, $\theta_{C L}$, a solution for $\beta$ was not found. From (4.9), when $a_{2}(\xi)=0$, only $\alpha$ could be determined, as presented in Figure 4.58, only one unknown existed and $\alpha$ was evaluated at all locations along the JCB as a function of $\theta$ (from Figure 4.18).

Across all cases, three main trends were observed. First, along the leading edge of the jet, $\alpha$ was greatest close to the floor and increased with elevation. Second, on the lee-side, the formation of counter-rotating vortices that accelerated flow around the jet created a rapidly increasing concentration of $\alpha$ at $\theta \sim 45$ and 315. And third, these regions exceeded the leading edge ranges for high cross-flow velocity. The 


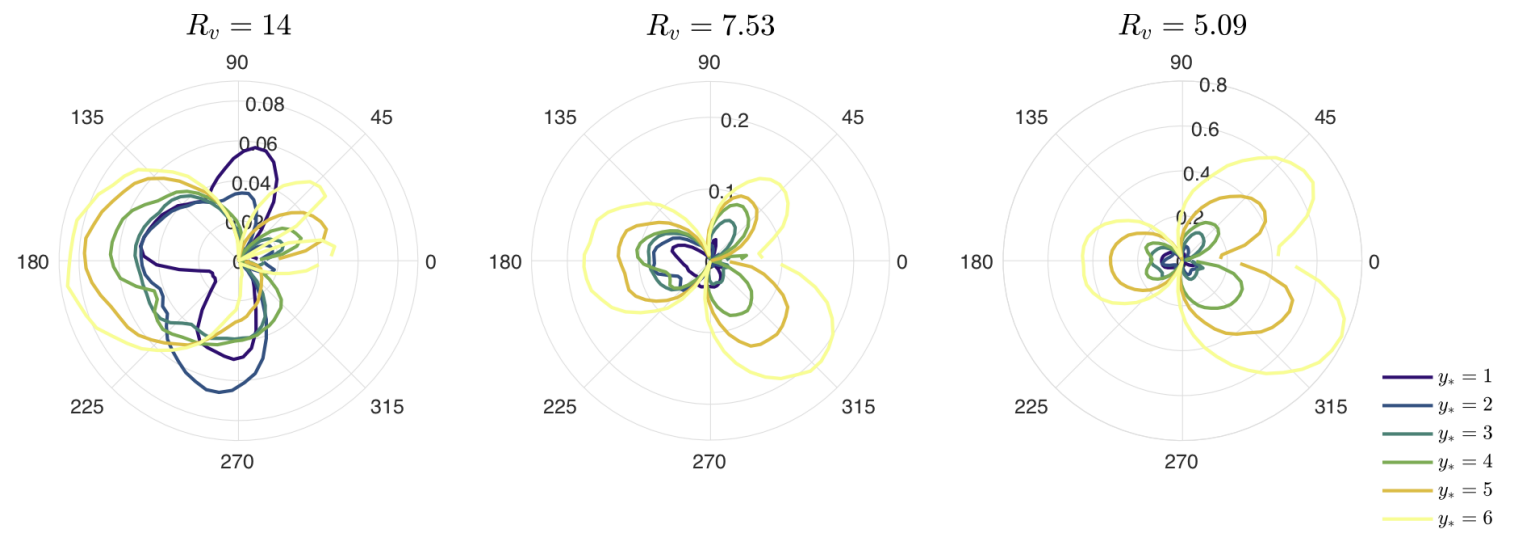

Figure 4.58: Entrainment coefficients in polar coordinates from $y_{*}=1 \rightarrow 6$. The leading edge of the jet is at $\theta=180$ for simplified recurring calculations.

ranges of $\alpha$ were found to be highly dependent on approaching inflow conditions. Near the floor, ranges of $0.04<\alpha<0.08$ were present for all cases due to the boundary layer. Increases in elevation and inflow velocity produced greater $\alpha$. These solutions provided a useful description of the entrainment dynamics, but were too complex and sensitive for such a simplified model. By way of example, at higher elevations of $R_{v}=5.09$ (right panel of Figure 4.58), $\alpha$ was found to exceed the extreme case values of $\alpha=0.15$ as cited by Hewett et al.[96].

As an alternative approach, a single coefficient was determined for each slice (similar to the analysis in Figure 4.57). The entrainment velocity was evaluated around the perimeter of the JCB and (4.9) was assessed at each height. Due to a nearly negligible deflection angle, all solutions of $\beta$ were negligible and only $\alpha$ was presented in Figure 4.59a. Similar to previous results (Figure 4.58), $\alpha$ was dependent on elevation and cross-flow velocity. For $R_{v}=14, \alpha$ is nearly constant and well within accepted values $(\alpha \approx 0.03)$. As the inflow velocity increased, $\alpha$ was negligible or negative. Without net entrainment (as was the case for the convection 

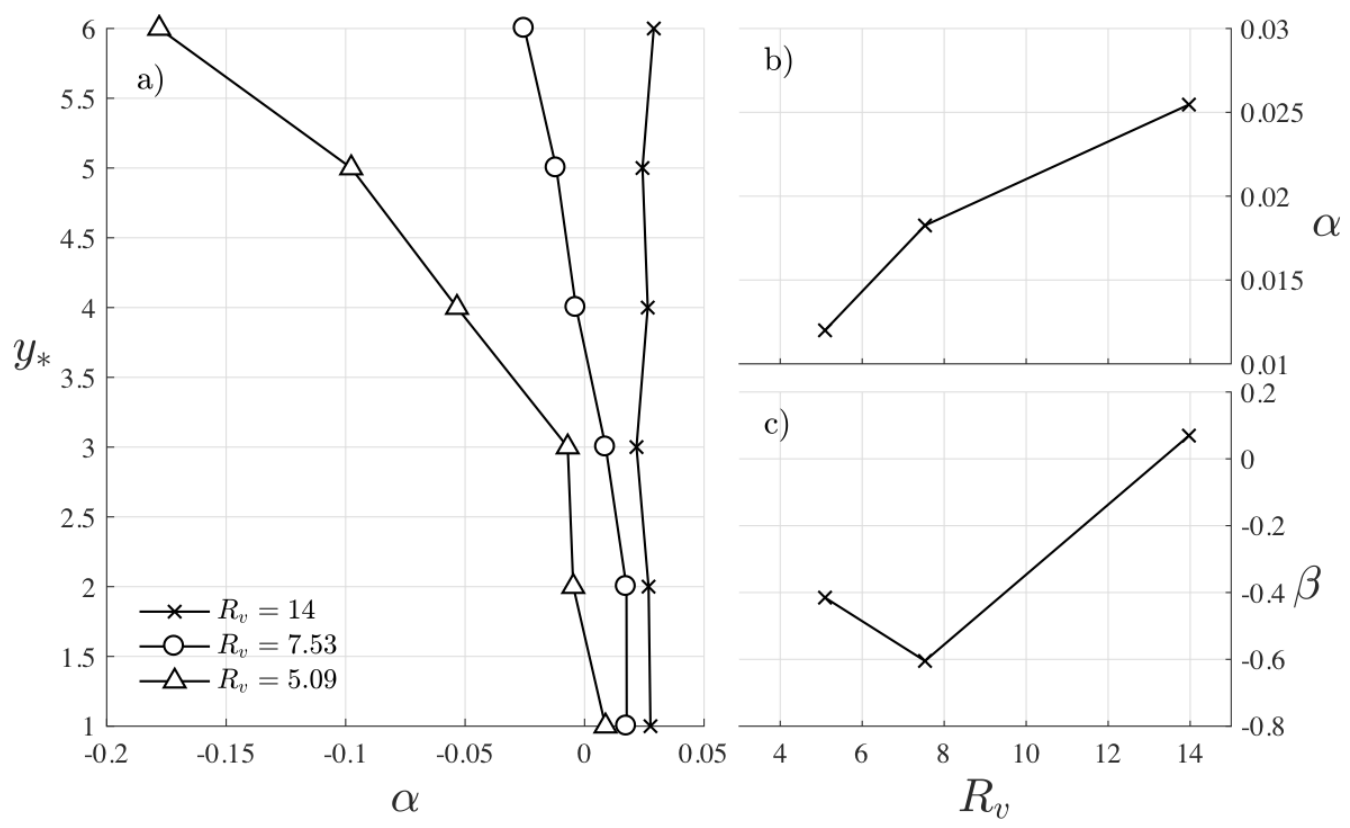

Figure 4.59: Entrainment coefficients extracted from the mean flow statistics a) as a function of elevation, and b) as a single solution for each case.

term in Figure 4.21), the closure terms must be compensated through negligible or negative solutions. On the center-plane, the out-of-plane dynamics observed in the $x-z$ plane were ignored, which yielded acceptable coefficients.

As part of the analysis to solve for all available elevations and produce a single solution, $\beta$ was reintroduced. For low cross-flow velocities, solutions using $\alpha=0.025$ and $\beta=0.07$ were closer but well below generally accepted values. For increased cross-flow velocity and the resultant increased deformation, $\beta$ was again negative. In total, this current method has proven to be ineffective in extracting entrainment coefficients. The near-field behavior could not be adequately compensated for due to the absence of inertial entrainment and asymmetric deformation. Therefore, to select the best approximation, direct calculations on the center-plane (from Figure 4.57) were investigated. 

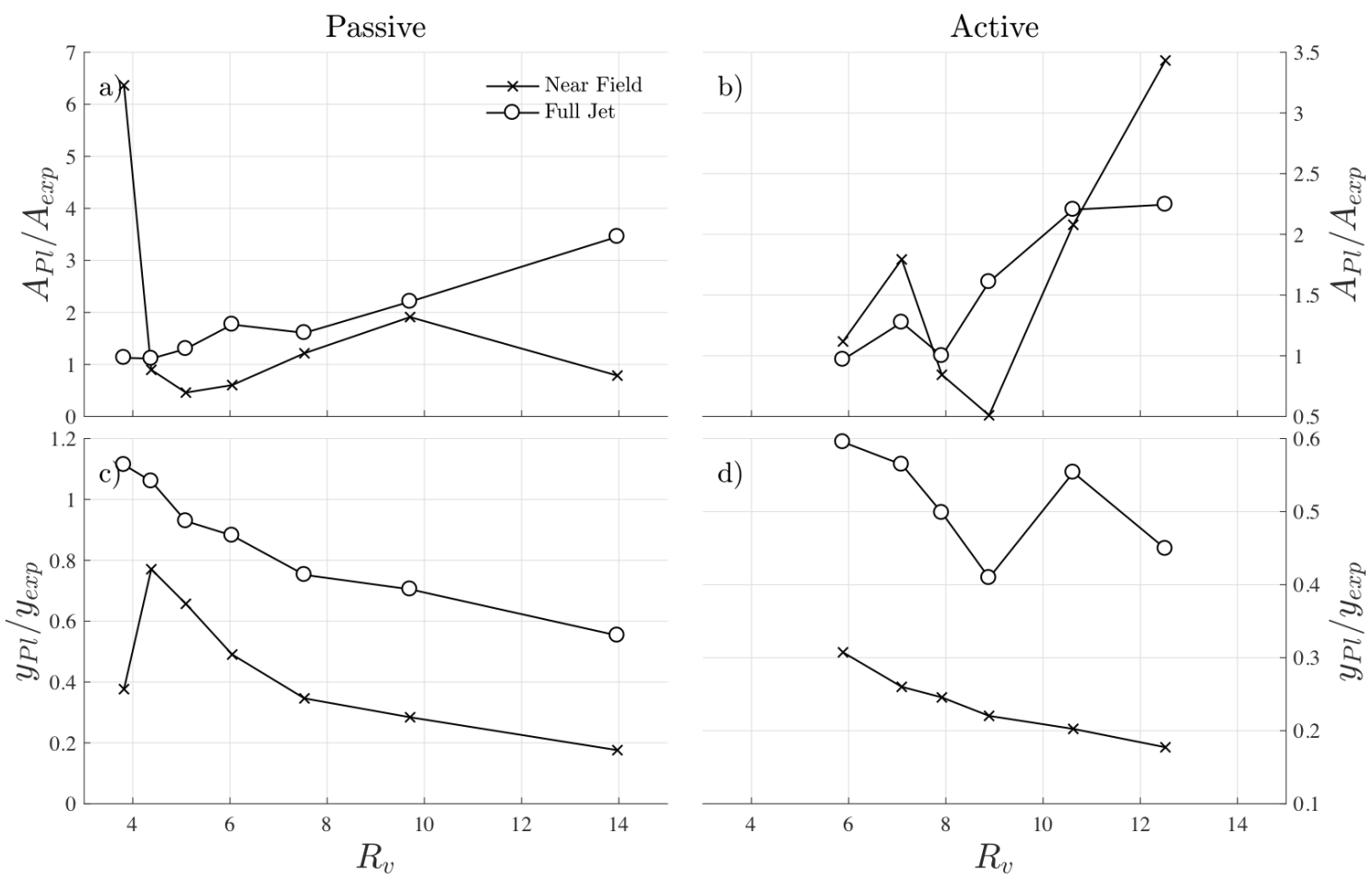

Figure 4.60: Comparison between the Plumeria outputs and experimental data for the cross-sectional area $(\mathrm{a}, \mathrm{b})$ and the final rise difference, $\Delta y,(\mathrm{c}, \mathrm{d})$ at the end of the experiment centerline.

\subsection{2 $\quad$ Extracted Closure Terms}

The range of computed closure terms from the center-plane were used as inputs for Plumeria to simulate identical conditions. To account for near-field behavior, the values for the full length of the jet and the near-field were separately evaluated. The model efficiency was determined through both the cross-plane area ratio, $A_{P l} / A_{\text {exp }}$, and the final rise height ratio, $y_{P l} / y_{e x p}$, where $X_{P l}$ and $X_{\text {exp }}$ indicated Plumeria and experimental data, respectively (Figure 4.60).

A comparison between center-plane cross-sectional areas (Figure 4.60a) indicated that Plumeria was able to reasonably predict the expansion in low inflow turbulence. 
The near-field accounted for the delay in entrainment, which was present until the expansion of the shear layer, yielding a better prediction of the expansion. For the full jet and far-field, the expansion of the shear layer overcompensated for the nearfield behavior, significantly over-predicting the entrainment and resulting expansion. Interestingly, the opposite effect was observed for the final rise height (Figure 4.60c). The near-field model, which uses a very high $\beta$, only rose to $\sim 20 \%$ of the true plume height. While improvements were found, the full jet predictions appeared to correctly predict both the area and final rise height for $R_{v} \approx 5$. Therefore, it can be concluded that compensation through consideration of near-field behavior suffered from other contributing factors.

For high inflow turbulence (Figure 4.60b and d), the dramatic increase in shear layer expansion after a region of constant cross-sectional area could not be accounted for accurately. Predictions for high $R_{v}$ were more than double the true area and rose less than $50 \%$ of the true height. While the full jet predictions were closer than the near-field, the presence of turbulent cross-flow added additional complexity that could not be accounted for in the current entrainment model. These results have also been observed for "slightly bent" plumes in Mastin [9]. The low deflection angle under-predicts $\beta$ contributions, which then rapidly increased after the final rise was achieved. These results revealed that a different methodology must be employed to reduce the dependence on the high $\beta$ extracted from the velocity fields.

\subsubsection{Summary}

Validation of reduced order closure terms for the one-dimensional model Plumeria was investigated through two methods. The first built upon the work of McNeal et 
al. [157], comparing output centerline trajectories to experimental data. For three inflow velocities, a range of $\alpha$ and $\beta$ were used to generate the centerline and plume radius. Residual analysis compared the trajectories and center-plane plume area using the JCB results from Chapter 4.1.3. For a each $\alpha$, the results provided an optimal $\beta$ that best matched experimental data.

Comparisons to experimental trajectories yielded reasonable ranges of entrainment coefficients well within accepted values. The addition of the plume area halved the optimal $\beta$, due to the added complexity of near-field behavior. The constant plume area near the floor changed the cross-flow entrainment to a correction term that would reduce entrainment for over-predictions in radial entrainment. Further analyses added volumetric comparisons utilizing the conic slices from the $x-z$ plane. These results identified that the constant area and negligible net entrainment had prevented accurate near-field predictions, overcompensating with negative $\beta$.

The coefficients determined from the center-plane shear layers were tested as inputs for Plumeria. The results identified compensation in the near-field that dramatically reduced the far-field efficiency. Additionally, the errors associated with "slightly bending" plumes from Mastin [9] appeared for $R_{v}>5$. Through this research, it became very clear that the simplicity of the current model could not account for near-field behavior, and that the contributions of additional parameters must be sought to fully capture the complexity in the Plumeria model. 


\section{Chapter 5}

\section{Conclusions}

The goal of this work was to explore simplified JICF models of fluids injected into oncoming cross-flow and quantify subsequent mixing as the jet was carried downstream. There are a host of reasons why it is beneficial to accurately predict JICF behavior, especially for optimizing engineering applications and predicting natural occurrences. The presented work was designed to test existing simplified volcanic

plume models, which present many significant uncertainties because they assume negligible turbulence. For the simplified model Plumeria, the underlying assumptions have been shown to yield significant errors for "slightly bent" plumes. This presents problems for two reasons: first, throughout literature, complex turbulent features have been found within the near-field where the plume develops and second, turbulence is prevalent throughout the atmosphere. The experiments covered in this work were designed to focus on the subtleties of the near-field interaction and decipher the contributions of turbulence, both within the jet and cross-flow, that simplified models have ignored.

For a range of inflow velocities with varying free-stream turbulence intensity, a large data set of instantaneous velocity fields was collected. These were decomposed to quantify the mean flow statistics and Reynolds stresses, each of which identified 
distinct differences when compared to the initial interaction, as the incoming crossflow turbulence intensity was increased. Most significantly, the mean flow-statistics identified variations in movement of cross-flow around the jet and alterations to the lee-side wake behavior. To further evaluate variations in behavior within the jet, the velocity data was transformed from the $x-y$ plane to relative components normal and tangential to the jet centerline. Once transformed, the Reynolds stresses identified two distinct features: the collapse of the potential core in the radial fluctuations and the edges of the shear layer (JCB) in the shear stress component. Scaling through the near-field factor $R_{v}^{2} D$ collapsed the data, thereby allowing for the description of physical features of the jet relative to the collapse of the core. This analysis identified the contributions of turbulent cross-flow, which increased the entrainment and dramatically increased the shear layer expansion.

The role of inflow conditions on inertial and Reynolds stress contributions were quantified through momentum and energy transport. Six $x-z$ PIV planes (parallel to the floor) were collected to provide a full description of the near-field behavior. Within the jet, the majority of momentum and energy losses occurred before the collapse of the core and turbulent entrainment process of viscous nibbling was observed in the Reynolds stress terms. The absence of net entrainment and near constant area of the jet close to the floor identified a distinct feature ignored in many simplified models. With turbulent cross-flow, dramatic changes to the wake behavior and recovery were observed, similar to descriptions of bluff body wakes in literature. These observations were confirmed through analysis of the wakes on $x-z$ planes, which identified additional changes to the turbulent vortex systems.

The significant variations in near-wake and near-field jet behavior required fur- 
ther investigation, specifically the changes to the vortex systems that contribute to entrainment. Decomposing the velocity field using snapshot POD identified the most energetic flow features within the jet, located in the lee-side wake region and followed by smaller contributions at the convergence of the shear layers. Additionally, an increase in inflow turbulence intensity added small features present in the cross-flow that contributed additional energy and significantly altered the description of the jet and JCB.

These small scale features were then quantified by evaluating the unresolved components through sub-grid models. Evaluation of a bulk eddy viscosity term provided details on the dissipation of energy and importance of turbulence in predicting these flows. The additional turbulence contributions present in the cross-flow were observed in the jet and increased the magnitude of the unresolved energy until the return to self-similarity in the far-field. Evaluation of the turbulent dissipation through the Modified Structure Function Method further quantified the energy present at the smallest scales. This confirmed the presence of energy in the smallest scale for AG cases further downstream.

The largest features within the flow were then investigated. Critical point analysis identified the vortex systems present along the leading edge of the jet and within the wake region. Critical points were classified by the surrounding flow structure, which confirmed the presence of the unstable focus near the floor and critical point pairs near vortices. The $Q$-criterion was used to classify critical points as vortices and allowed calculations of the area and circulation. The significant quantity of these features leaves significant room for additional analysis.

Comparisons of coherent structures throughout the interaction using two-point 
correlation functions confirmed the increased wake recovery for high inflow turbulence. Increased coherence throughout the wake region and within the lee-side of the jet identified that similar features within the wake were entrained by the jet. The anti-correlations with the leading edge and extended coherence near the shear layer vortices identified two different flow structures for high inflow turbulence. The leading edge vortices, which continuously evolved and did not correlate for PG, expanded into the cross-flow which allowed the structures inside to persist through the jet. The strong separation between the leading edge and lee-side indicated a reduction in mixing that could be explained by the dissipation of the counter-rotating vortices.

Finally, these results were used to test the simplified model, Plumeria. Summarizing the entrainment through two closure terms, the predicted plume shapes were compared to experimental data. This amplified the inability for Plumeria to account for the near-field behavior and turbulent features. The near-field constant area and rapid expansion also conflicted, yielding either results that emphasized efficient plume rise models or results optimized to demonstrate shear layer expansion. Therefore, in future studies, additional considerations must be included to detect, understand and accurately account for the near-field behavior.

These observations provided a complete description of the role of cross-flow turbulence on the behavior of JICF. Most importantly, this research highlighted the negligible entrainment near the exit and impact of small scale turbulent features that must be included for any simplified model to yield reasonable predictions. By laying the foundation for a more nuanced approach to JICF research, it is possible to more precisely summarize the complex features observed in this work through sim- 
plified descriptions that can be of benefit to both engineering design and geophysical modelling. 


\section{Chapter 6}

\section{Future Work}

\subsection{Low Order Model Comparisons}

From section 4.7, the closure terms for the reduced order model Plumeria have been computed directly from experimental data and optimized through comparisons to experimental centerline, area or volume data. Following current theoretical models, these methods did not compensate for near-field behavior or high inflow turbulence. Consequently, the dependence on a single coefficient yielded negative closure terms to account for the constant volume through three diameters, which over-predicted the volume and bending of the jet significantly. In addition, the assumption of axisymmetric expansion did not account for the out-of-plane deformations, which were present until self-similarity returned in the far-field. Attempts to separate regions for analysis resulted in predictions that did not align with the experimental data.

From Mastin [9], the behavior of "slightly bent" plumes created significant errors due to the drastically reduced deflection angle before the final plume height was reached (see Figure 6.1). Similar results were found in section 4.7.2. Therefore, the current model could not compensate for complex turbulent features, and the 


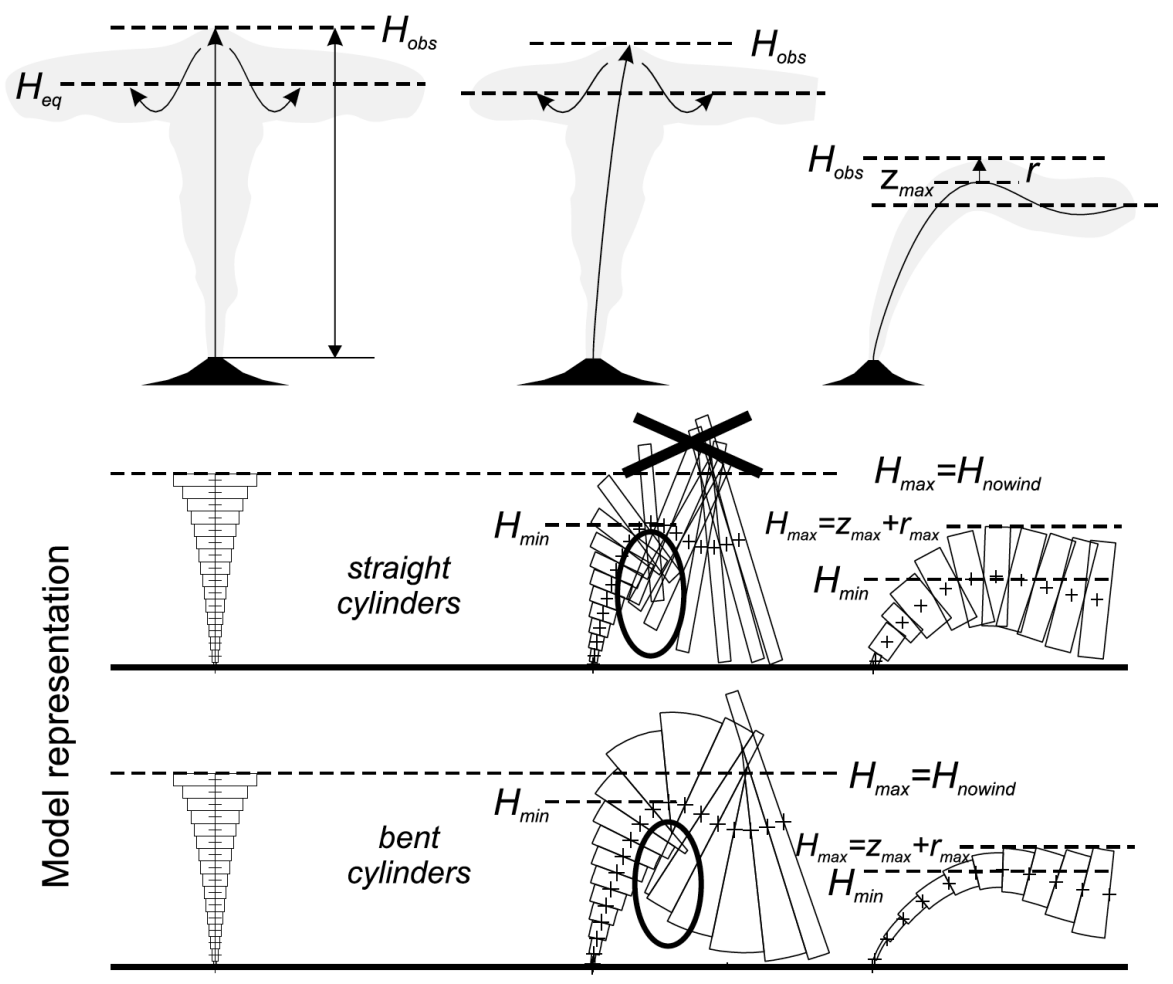

Figure 6.1: Simplified model complications for bending plume from Mastin [9, fig. 1].

near-field behavior must be compensated for through additional descriptions of that interaction.

From the definition of the entrainment velocity, (Equation 2.17) $u_{e}=$ $\alpha\left|u_{c}-u_{\infty} \cos \theta_{C L}\right|+\beta\left|u_{\infty} \sin \theta_{C L}\right|$ utilized both the behavior of the jet and the cross-flow in tangent with the radial entrainment term $\alpha$. The $\beta$ term, which had a large impact on both plume growth and centerline deflection, only accounted for the cross-flow after the jet had bent downstream. As a result, the next stage of this analysis will focus on accounting for the negligible near-field entrainment observed in Figure 4.21. Three trends will also be investigated for their contributions in the 
subsections below.

\section{Lee-side Wake and Secondary Flow Contributions}

The secondary flow observed on the lee-side of the jet in the PG cases is a product of the turbulent behavior of JICF. The flow entrained in the recirculation region creates a pressure change that allows the counter-rotating vortices to form. This entrained flow is then carried along the lee-side of the jet and entrained. These features are muted in higher turbulence intensities. Therefore, to quantify these contributions, the secondary flow properties (and reduction for AG) will be investigated and compared across cases.

\section{Additional Inflow Considerations}

From Costa et al. [90], a variety of simplified plume models were tested to determine their efficiency. One of the inputs incorporated atmospheric conditions, including the wind velocity at varying elevations (Costa et al. [90, fig. 1]). From this research, it is clear that the analyses must also consider the role of turbulent inflow as a significant influence on the entrainment and expansion of the shear layer. In Riedel \& Sitzmann [158], the measurements of the atmospheric turbulence intensity collected in flight found a dependence on wind speed that was similar to previous wind tunnel experiments. Therefore, further investigations will relate the inflow turbulence intensity profile (from Figure 3.4) and the resulting change in entrainment. 


\section{Near-field Compensation and Scaling}

From section 4.1.2, the power law coefficients and location of the collapse of the potential core were described through the near-field scaling term $R_{v}^{2} D$. With this insight, a description of the near-field using these relationships may allow for the delayed expansion and negligible entrainment. An addition to the cross-flow term is proposed, one that would neutralize $\alpha$ when $\xi / \xi^{0}<1$, enhancing these features of the jet. While this relationship was already present in the $\beta$ term through the $u_{\infty} \sin \theta_{C L}$, the impact was negligible at low deflections angles.

\subsection{Vortex System Entrainment Contributions}

From the results in section 4.5, the identification of critical points along the leading edge of the jet and lee-side wake revealed the presence of features from two of the vortex systems. Classification of the surrounding flow further identified critical point pairs present in the flow that entrain surrounding fluid (Figure 4.46). Calculation of the second invariant, known as the $Q$-criterion, allowed for a measurement of the vortex sizes by isolating positive peaks with critical points.

In future work, analyses of these results will be focused on two physical properties. First, the circulation of the vortices, $\Gamma$, defined as $\Gamma=\oint \omega \mathrm{d} s$, will be determined for each vortex and compared across inflow conditions. The development and decay of the leading edge vortices will be quantified as a function of jet length. With the results obtained in section 4.4 .2 and 4.4.3, the turbulent properties will be examined to provide ranges of scales that will aid reduced order models and increase their predictive behavior.

Within the wake, the shed vortices entrain boundary layer fluid, which were then 


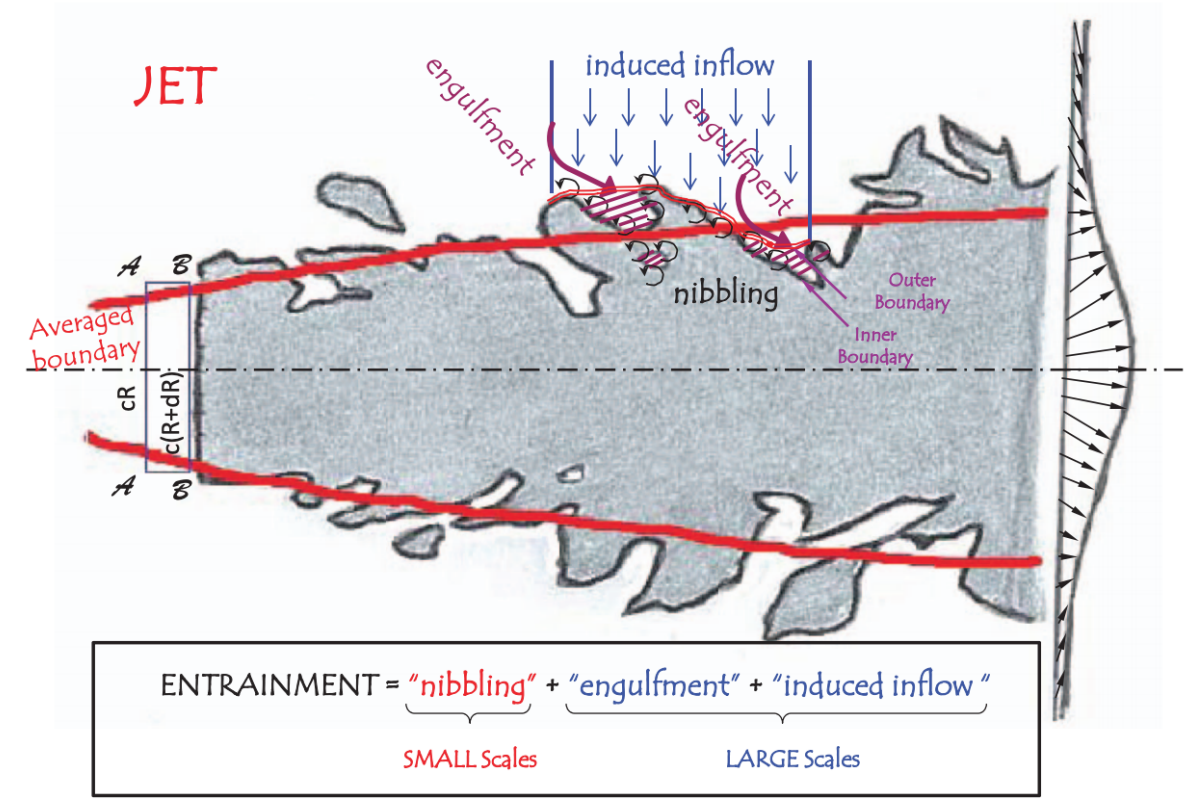

Figure 6.2: Cartoon of entrainment processes at the edge of the shear layer of a jet from Philip \& Marusic [10, fig. 6].

entrained by the counter-rotating vortices. The approximate location of the wake vorticies contacting the JCB had been noted in previous results (Figure 4.13). The movement of fluid vertically from the floor had been observed in mean flow statistics (Figure 4.17) and were measured within the boundary of the wake vortices identified in section 4.5.2. Therefore, further investigation to identify the wake vortices and measure the transport of fluid vertically will allow for the quantification of this source of entrainment.

To expand on this analysis, these shear layer vortices will be used to quantify the entrainment of surrounding flow by large scale engulfment. In Figure 6.2, the superposition of the mean shear layer edge over the instantaneous edge allows for the quantification of surrounding of the flow that has been "engulfed" (red striped area at top of Figure 6.2). This was previously investigated for the mean transport 
in section 4.6.1. From the traced shape of the vortices and evaluation of the raw PIV images, the instantaneous boundary of the jet will be defined. The cross-flow within the mean JCB will then be measured and a quantification of engulfment will be performed through transport analysis. While the contributions of viscous nibbling cannot be measured through PIV data, a quantified comparison between the net entrained momentum and energy will allow for the approximate contributions of small scale entrainment.

\subsection{Buoyant Plumes}

Unlocking how a jet of air mixes with air under a variety of conditions has seemingly unlimited applications for solving pressing real world engineering and environmental problems. From volcanic plume models to coolant transport, the complexity of this phenomenon involves interactions between varying density mediums. For this reason, analysis of positively and negatively buoyant plumes will provide important details on how a variety of gases interact with cross-flow.

The pilot experiments conducted in the Portland State University wind tunnel in 2018 compared jets of helium (positively buoyant) and argon (negatively buoyant) for a range of inflow velocities. Expanding on the work already completed (see section 3.4), additional cases will be investigated for similar scaling parameters and will undergo entrainment analyses. In addition, these gases will be used to investigate the the empirical component $n$ (Equation (2.18)) and the density term (Equation (2.19)) described in section 2.2. Ultimately, Plumeria data will be generated for a wider variety of conditions, and trajectory and jet width comparisons will be made. This basic research will add to our foundational understanding of JICF, 
and will move the field one step closer to scientific breakthroughs in engineering design efficiencies and predictive models that will impact millions of lives. 


\section{References}

[1] R. E. Holasek and S. Self. GOES weather satellite observations and measurements of the May 18, 1980, Mount St. Helens eruption. Journal of Geophysical Research: Solid Earth, 100(B5):8469-8487, 1995.

[2] D. K. Bisset, J. C. R. Hunt, and M. M. Rogers. The turbulent/non-turbulent interface bounding a far wake. Journal of Fluid Mechanics, 451:383-410, January 2002.

[3] T. F. Fric and A. Roshko. Vortical structure in the wake of a transverse jet. Journal of Fluid Mechanics, 279:1, November 1994.

[4] S. L. V. Coelho and J. C. R. Hunt. The dynamics of the near field of strong jets in crossflows. Journal of Fluid Mechanics, 200:95-120, March 1989.

[5] L. G. Mastin, M. Guffanti, R. Servranckx, P. Webley, S. Barsotti, K. Dean, A. Durant, J.W. Ewert, A. Neri, W.I. Rose, D. Schneider, L. Siebert, B. Stunder, G. Swanson, A. Tupper, A. Volentik, and C.F. Waythomas. A multidisciplinary effort to assign realistic source parameters to models of volcanic ash-cloud transport and dispersion during eruptions. Journal of Volcanology and Geothermal Research, 186(1-2):10-21, September 2009.

[6] D. Xu and J. Chen. Accurate estimate of turbulent dissipation rate using PIV data. Experimental Thermal and Fluid Science, 44:662-672, January 2013.

[7] A. E. Perry and M. S. Chong. A Description of Eddying Motions and Flow Patterns Using Critical-Point Concepts. Annual Review of Fluid Mechanics, 19(1):125-155, 1987.

[8] L.G. Mastin. A user-friendly one-dimensional model for wet volcanic plumes. Geochemistry, Geophysics, Geosystems, 8(3), 2007.

[9] L. G. Mastin. Testing the accuracy of a 1-D volcanic plume model in estimating mass eruption rate. Journal of Geophysical Research: Atmospheres, 119(5):2013JD020604, March 2014. 
[10] J. Philip and I. Marusic. Large-scale eddies and their role in entrainment in turbulent jets and wakes. Physics of Fluids, 24(5):055108, May 2012. Publisher: American Institute of Physics.

[11] F. Bazdidi-Tehrani, A. Shahmir, and A. Haghparast-Kashani. Numerical analysis of a single row of coolant jets injected into a heated crossflow. Journal of Computational and Applied Mathematics, 168(1):53-63, July 2004.

[12] Asif Hoda and Sumanta Acharya. Predictions of a Film Coolant Jet in Crossflow With Different Turbulence Models. Journal of Turbomachinery, 122(3):558-569, July 2000. Publisher: American Society of Mechanical Engineers Digital Collection.

[13] G. A. Briggs. Optimum Formulas for Buoyant Plume Rise. Philosophical Transactions of the Royal Society of London. Series A, Mathematical and Physical Sciences, 265(1161):197-203, 1969. Publisher: The Royal Society.

[14] G. A. Briggs. Chimney plumes in neutral and stable surroundings. Atmospheric Environment, 6(7):507-510, July 1972.

[15] Nejla Mahjoub Saïd, Hatem Mhiri, Georges Le Palec, and Philippe Bournot. Experimental and numerical analysis of pollutant dispersion from a chimney. Atmospheric Environment, 39(9):1727-1738, March 2005.

[16] R. C. Y. Koh and N. H. Brooks. Fluid Mechanics of Waste-Water Disposal in the Ocean. Annual Review of Fluid Mechanics, 7:187-211, 1975.

[17] L. Wilson, R. S. J. Sparks, T. C. Huang, and N. D. Watkins. The control of volcanic column heights by eruption energetics and dynamics. Journal of Geophysical Research: Solid Earth, 83(B4):1829-1836, 1978.

[18] R. J. Margason. Fifty years of jet in cross flow research. volume 534 of $A G A R D$ Conference Proceedings AGARD CP, pages 1-2, Winchester, November 1993. NATO.

[19] J. F. Keffer and W. D. Baines. The round turbulent jet in a cross-wind. Journal of Fluid Mechanics, 15(04):481-496, April 1963.

[20] G. A. Briggs. A Plume Rise Model Compared with Observations. Journal of the Air Pollution Control Association, 15(9):433-438, September 1965.

[21] B. D. Pratte and W. D. Baines. Profiles of the Round Turbulent Jet in A Cross Flow. Journal of the Hydraulics Division, 93(6):53-64, 1967. 
[22] R. J. Margason. The path of a jet directed at large angles to a subsonic free stream. NASA Technical Note NASA TN D-4919, Langley Research Center, National Aeronautics and Space Administration, 1968.

[23] Y. Kamotami and I. Greber. Experiments on a Turbulent Jet in a Cross Flow. AIAA Journal, 10(11):1425-1429, 1972.

[24] P. Chassaing, J. George, A. Claria, and F. Sananes. Physical characteristics of subsonic jets in a cross-stream. Journal of Fluid Mechanics, 62(01):41-64, January 1974.

[25] J. Sucec and W. W. Bowley. Prediction of the Trajectory of a Turbulent Jet Injected Into a Crossflowing Stream. Journal of Fluids Engineering, 98(4):667672, December 1976.

[26] S. V. Patankar, D. K. Basu, and S. A. Alpay. Prediction of the ThreeDimensional Velocity Field of a Deflected Turbulent Jet. Journal of Fluids Engineering, 99(4):758-762, December 1977.

[27] W. K. George and H. J. Hussein. Locally axisymmetric turbulence. Journal of Fluid Mechanics, 233:1-23, December 1991.

[28] W. K. George and M. M. Gibson. The self-preservation of homogeneous shear flow turbulence. Experiments in Fluids, 13(4):229-238, August 1992.

[29] H. J. Hussein, S. P. Capp, and W. K. George. Velocity measurements in a high-Reynolds-number, momentum-conserving, axisymmetric, turbulent jet. Journal of Fluid Mechanics, 258:31-75, January 1994.

[30] S. B. Pope. Turbulent Flows. Measurement Science and Technology, 12(11):2020, 2001.

[31] S. H. Smith and M. G. Mungal. Mixing, structure and scaling of the jet in crossflow. Journal of Fluid Mechanics, 357:83-122, February 1998.

[32] H. E. Fiedler. Coherent structures in turbulent flows. Progress in Aerospace Sciences, 25(3):231-269, January 1988.

[33] A. A. Townsend. The properties of equilibrium boundary layers. Journal of Fluid Mechanics, 1(6):561-573, December 1956. Publisher: Cambridge University Press.

[34] A. N. Kolmogorov. Dissipation of Energy in Locally Isotropic Turbulence. Akademiia Nauk SSSR Doklady, 32:16, April 1941. 
[35] W. K. George. Lectures in Turbulence for the 21st Century.

[36] S. Corrsin and A. L. Kistler. Free-Stream Boundaries of Turbulent Flows. January 1955.

[37] C. B. da Silva and R. R. Taveira. The thickness of the turbulent/nonturbulent interface is equal to the radius of the large vorticity structures near the edge of the shear layer. Physics of Fluids, 22(12):121702, December 2010.

[38] G. L. Brown and A. Roshko. On density effects and large structure in turbulent mixing layers. Journal of Fluid Mechanics, 64(4):775-816, July 1974.

[39] W. J. A. Dahm and P. E. Dimotakis. Measurements of entrainment and mixing in turbulent jets. AIAA Journal, 25(9):1216-1223, 1987.

[40] D. Liepmann and M. Gharib. The role of streamwise vorticity in the nearfield entrainment of round jets. Journal of Fluid Mechanics, 245:643-668, December 1992.

[41] J. Mathew and A. J. Basu. Some characteristics of entrainment at a cylindrical turbulence boundary. Physics of Fluids, 14(7):2065-2072, May 2002.

[42] J. Westerweel, C. Fukushima, J. M. Pedersen, and J. C. R. Hunt. Mechanics of the Turbulent-Nonturbulent Interface of a Jet. Physical Review Letters, 95(17):174501, October 2005.

[43] M. Holzner and B. Luthi. Laminar Superlayer at the Turbulence Boundary. Physical Review Letters, 106(13):134503, March 2011.

[44] T. S. Silva, M. Zecchetto, and C. B. da Silva. The scaling of the turbulent/nonturbulent interface at high Reynolds numbers. Journal of Fluid Mechanics, 843:156-179, May 2018.

[45] M. Wolf, B. Lüthi, M. Holzner, D. Krug, W. Kinzelbach, and A. Tsinober. Investigations on the local entrainment velocity in a turbulent jet. Physics of Fluids, 24(10):105110, October 2012.

[46] R. R. Taveira, J. S. Diogo, D. C. Lopes, and C. B. da Silva. Lagrangian statistics across the turbulent-nonturbulent interface in a turbulent plane jet. Physical Review E, 88(4):043001, October 2013.

[47] D. Mistry, J. Philip, J. R. Dawson, and I. Marusic. Entrainment at multi-scales across the turbulent/non-turbulent interface in an axisymmetric jet. Journal of Fluid Mechanics, 802:690-725, September 2016. 
[48] S. Megerian, J. Davitian, L. S. D. B. Alves, and A. R. Karagozian. Transversejet shear-layer instabilities. Part 1. Experimental studies. Journal of Fluid Mechanics, 593:93-129, December 2007.

[49] A. Glezer. The formation of vortex rings. Physics of Fluids (1958-1988), 31(12):3532-3542, December 1988.

[50] H. A. Becker and T. A. Massaro. Vortex evolution in a round jet. Journal of Fluid Mechanics, 31(3):435-448, February 1968.

[51] E. Gutmark and C. M. Ho. Preferred modes and the spreading rates of jets. The Physics of Fluids, 26(10):2932-2938, October 1983.

[52] A. R. Karagozian. An analytical model for the vorticity associated with a transverse jet. AIAA Journal, 24(3):429-436, 1986.

[53] L. Cortelezzi and A. R. Karagozian. On the formation of the counter-rotating vortex pair in transverse jets. Journal of Fluid Mechanics, 446:347-373, November 2001.

[54] L. K. Su and M. G. Mungal. Simultaneous measurements of scalar and velocity field evolution in turbulent crossflowing jets. Journal of Fluid Mechanics, 513:1-45, August 2004.

[55] T. H. New, T. T. Lim, and S. C. Luo. Effects of jet velocity profiles on a round jet in cross-flow. Experiments in Fluids, 40(6):859-875, June 2006.

[56] R. A. Antonia and Q. Zhao. Effect of initial conditions on a circular jet. Experiments in Fluids, 31(3):319-323, September 2001.

[57] M. Breda and O. R. H. Buxton. Influence of coherent structures on the evolution of an axisymmetric turbulent jet. Physics of Fluids, 30(3):035109, March 2018.

[58] A. Krothapalli, L. Lourenco, and J. M. Buchlin. Separated flow upstream of a jet in a crossflow. AIAA Journal, 28(3):414-420, 1990.

[59] R. M. Kelso, T. T. Lim, and A. E. Perry. An experimental study of round jets in cross-flow. Journal of Fluid Mechanics, 306:111-144, January 1996.

[60] P. B. V. Johansson and W. K. George. The far downstream evolution of the high-Reynolds-number axisymmetric wake behind a disk. Part 1. Single-point statistics. Journal of Fluid Mechanics, 555:363-385, May 2006. Publisher: Cambridge University Press. 
[61] H. Higuchi, R. W. Anderson, and J. Zhang. Three-dimensional wake formations behind a family of regular polygonal plates. AIAA Journal, 34(6):11381145, 1996. Publisher: American Institute of Aeronautics and Astronautics.

[62] J. C. Hu and Y. Zhou. Aerodynamic Characteristics of Asymmetric Bluff Bodies. Journal of Fluids Engineering, 131(1), January 2009. Publisher: American Society of Mechanical Engineers Digital Collection.

[63] J. Nedić, J. C. Vassilicos, and B. Ganapathisubramani. Axisymmetric Turbulent Wakes with New Nonequilibrium Similarity Scalings. Physical Review Letters, 111(14):144503, October 2013. Publisher: American Physical Society.

[64] J. Nedić, B. Ganapathisubramani, and J. C. Vassilicos. Drag and near wake characteristics of flat plates normal to the flow with fractal edge geometries. Fluid Dynamics Research, 45(6):061406, August 2013. Publisher: IOP Publishing.

[65] J. Nedić, O. Supponen, B. Ganapathisubramani, and J. C. Vassilicos. Geometrical influence on vortex shedding in turbulent axisymmetric wakes. Physics of Fluids, 27(3):035103, March 2015. Publisher: American Institute of Physics.

[66] R. I. Sykes, W. S. Lewellen, and S. F. Parker. On the vorticity dynamics of a turbulent jet in a crossflow. Journal of Fluid Mechanics, 168:393-413, July 1986.

[67] E. F. Hasselbrink and M. G. Mungal. Transverse jets and jet flames. Part 1. Scaling laws for strong transverse jets. Journal of Fluid Mechanics, 443:1-25, September 2001.

[68] G. I. Taylor. Experiments with rotating fluids. Proceedings of the Royal Society of London. Series A, Containing Papers of a Mathematical and Physical Character, 100(703):114-121, November 1921.

[69] S. A. Orszag. Analytical theories of turbulence. Journal of Fluid Mechanics, 41(2):363-386, April 1970.

[70] F. H. Harlow and J. E. Welch. Numerical Calculation of Time-Dependent Viscous Incompressible Flow of Fluid with Free Surface. The Physics of Fluids, 8(12):2182-2189, December 1965.

[71] K. Aziz and J. D. Hellums. Numerical Solution of the Three-Dimensional Equations of Motion for Laminar Natural Convection. The Physics of Fluids, 10(2):314-324, February 1967. 
[72] A. J. Chorin. Numerical solution of the Navier-Stokes equations. Mathematics of Computation, 22(104):745-762, 1968.

[73] D. K. Lilly and H. H. Goldstine. Proceedings of the IBM Scientific Computing Symposium on Environmental Sciences. IBM, 1967.

[74] C. W. Hirt. Computer Studies of Time-Dependent Turbulent Flows. The Physics of Fluids, 12(12):II-219, December 1969.

[75] J. W. Deardorff. A numerical study of three-dimensional turbulent channel flow at large Reynolds numbers. Journal of Fluid Mechanics, 41(2):453-480, April 1970.

[76] J. Boussinesq. Theorie de l'ecoulement tourbillant. Mem. Acad. Sci., 23:46, 1877.

[77] F. G. Schmitt. About Boussinesq's turbulent viscosity hypothesis: historical remarks and a direct evaluation of its validity. Comptes Rendus Mécanique, 335(9):617-627, September 2007.

[78] B. E. Launder and D. B. Spalding. The numerical computation of turbulent flows. Computer Methods in Applied Mechanics and Engineering, 3(2):269289, March 1974.

[79] J. Smagorinsky. General circulation experiments with the primitive equations. Monthly Weather Review, 91(3):99-164, March 1963.

[80] D. Crabb, D. F. G. Durao, and J. H. Whitelaw. A Round Jet Normal to a Crossflow. Journal of Fluids Engineering, 103(1):142-153, March 1981.

[81] J. Andreopoulos and W. Rodi. Experimental investigation of jets in a crossflow. Journal of Fluid Mechanics, 138:93-127, January 1984.

[82] M. K. W. Wille. Large Eddy Simulation of jets in cross-flows. PhD thesis, Imperial College London (University of London), 1997.

[83] B. Wegner, Y. Huai, and A. Sadiki. Comparative study of turbulent mixing in jet in cross-flow configurations using LES. International Journal of Heat and Fluid Flow, 25(5):767-775, October 2004.

[84] S. M. Dash, D. C. Kenzakowski, J. M. Seiner, and T. R. S. Bhat. Recent advances in jet flowfield simulation. I - Steady flows. In 15th Aeroacoustics Conference, Long Beach, California, 1993. American Institute of Aeronautics and Astronautics. 
[85] L. L. Yuan and R. L. Street. Trajectory and entrainment of a round jet in crossflow. Physics of Fluids, 10(9):2323-2335, September 1998.

[86] G. Chochua, D. Lischinsky, W. Shyy, S. Thakur, A. Brankovic, J. Lienau, and L. Porter. A Computational and Experimental Investigation of Turbulent Jet and Crossflow Interaction. Numerical Heat Transfer, Part A: Applications, 38(6):557-572, November 2000.

[87] N. A. Durando. Vortices induced in a jet by a subsonic cross flow. AIAA Journal, 9(2):325-327, 1971.

[88] D. P. Hoult and J. C. Weil. Turbulent plume in a laminar cross flow. Atmospheric Environment (1967), 6(8):513-531, August 1972.

[89] M. Bursik. Effect of wind on the rise height of volcanic plumes. Geophysical Research Letters, 28(18):3621-3624, 2001.

[90] A. Costa, Y. J. Suzuki, M. Cerminara, B. J. Devenish, T. Esposti Ongaro, M. Herzog, A. R. Van Eaton, L. C. Denby, M. Bursik, M. de' Michieli Vitturi, S. Engwell, A. Neri, S. Barsotti, A. Folch, G. Macedonio, F. Girault, G. Carazzo, S. Tait, E. Kaminski, L. G. Mastin, M. J. Woodhouse, J. C. Phillips, A. J. Hogg, W. Degruyter, and C. Bonadonna. Results of the eruptive column model inter-comparison study. Journal of Volcanology and Geothermal Research, 326:2-25, October 2016.

[91] B. R. Morton, G. Taylor, and J. S. Turner. Turbulent gravitational convection from maintained and instantaneous sources. In Proceedings of the Royal Society of London A: Mathematical, Physical and Engineering Sciences, volume 234, pages 1-23. The Royal Society, 1956.

[92] G. A. Briggs. Plume Rise Predictions. In Lectures on Air Pollution and Environmental Impact Analyses, pages 59-111. American Meteorological Society, 1982.

[93] F. P. Ricou and D. B. Spalding. Measurements of entrainment by axisymmetrical turbulent jets. Journal of Fluid Mechanics, 11(1):21-32, August 1961.

[94] A. M. Falcone and J. C. Cataldo. Entrainment Velocity in an Axisymmetric Turbulent Jet. Journal of Fluids Engineering, 125(4):620, 2003.

[95] S. A. Solovitz and L. G. Mastin. Experimental study of near-field air entrainment by subsonic volcanic jets. Journal of Geophysical Research, 114(B10), October 2009. 
[96] T. A. Hewett, J. A. Fay, and D. P. Hoult. Laboratory experiments of smokestack plumes in a stable atmosphere. Atmospheric Environment (1967), 5(9):767-789, September 1971.

[97] R. Jordinson. Flow in a jet directed normal to the wind. Ministry of Supply, Aeronautical Research Council, London, 1956. Open Library ID: OL21500225M.

[98] D. P. Hoult, J. A. Fay, and L. J. Forney. A Theory of Plume Rise Compared with Field Observations. Journal of the Air Pollution Control Association, 19(8):585-590, August 1969.

[99] P. Huq and E. J. Stewart. A laboratory study of buoyant plumes in laminar and turbulent crossflows. Atmospheric Environment, 30(7):1125-1135, April 1996.

[100] Y. J. Suzuki and T. Koyaguchi. Effects of wind on entrainment efficiency in volcanic plumes: wind effects on volcanic plume dynamics. Journal of Geophysical Research: Solid Earth, 120(9):6122-6140, September 2015.

[101] M. Bursik, M. Jones, S. Carn, K. Dean, A. Patra, M. Pavolonis, E. B. Pitman, T. Singh, P. Singla, P. Webley, H. Bjornsson, and M. Ripepe. Estimation and propagation of volcanic source parameter uncertainty in an ash transport and dispersal model: application to the Eyjafjallajokull plume of 14-16 April 2010. Bulletin of Volcanology, 74(10):2321-2338, December 2012.

[102] M. J. Woodhouse, A. J. Hogg, J. C. Phillips, and R. S. J. Sparks. Interaction between volcanic plumes and wind during the 2010 Eyjafjallajökull eruption, Iceland. Journal of Geophysical Research: Solid Earth, 118(1):92-109, January 2013.

[103] Simmons L. F. G. and Salter C. Experimental investigation and analysis of the velocity variations in turbulent flow. Proceedings of the Royal Society of London. Series A, Containing Papers of a Mathematical and Physical Character, 145(854):212-234, June 1934.

[104] E. A. Novikov. Intermittency and scale similarity in the structure of a turbulent plow. Journal of Applied Mathematics and Mechanics, 35(2):231-241, January 1971.

[105] B. B. Mandelbrot. Intermittent turbulence in self-similar cascades: divergence of high moments and dimension of the carrier. Journal of Fluid Mechanics, 62(2):331-358, January 1974. 
[106] U. Frisch, P. L. Sulem, and M. Nelkin. A simple dynamical model of intermittent fully developed turbulence. Journal of Fluid Mechanics, 87(4):719-736, August 1978.

[107] T. S. Lundgren. Strained spiral vortex model for turbulent fine structure. The Physics of Fluids, 25(12):2193-2203, December 1982.

[108] D. Queiros-Conde and J. C. Vassilicos. Turbulent Wakes of 3D Fractal Grids. In Intermittency in Turbulent Flows, volume 12 of 11, pages 136-167. Cambridge University Press, 1 edition, 2000.

[109] B. Mazzi, F. Okkels, and J. C. Vassilicos. A shell-model approach to fractalinduced turbulence. The European Physical Journal B - Condensed Matter and Complex Systems, 28(2):243-251, July 2002.

[110] M. Gad-El-Hak and S. Corrsin. Measurements of the nearly isotropic turbulence behind a uniform jet grid. Journal of Fluid Mechanics, 62(1):115-143, January 1974.

[111] Y. Tassa and Y. Kamotani. Experiments on turbulence behind a grid with jet injection in downstream and upstream direction. The Physics of Fluids, 18(4):411-414, April 1975.

[112] S. C. Ling and C. A. Wan. Decay of Isotropic Turbulence Generated by a Mechanically Agitated Grid. The Physics of Fluids, 15(8):1363-1369, August 1972 .

[113] K. Sato and Y. Saito. Unstable Cavitation Behavior in a Circular-Cylindrical Orifice Flow. January 2001.

[114] H. Makita and K. Sassa. Active Turbulence Generation in a Laboratory Wind Tunnel. In A. V. Johansson and P. H. Alfredsson, editors, Advances in Turbulence 3, pages 497-505. Springer Berlin Heidelberg, January 1991.

[115] A. Talamelli, L. Riparbelli, and J. Westin. An active grid for the simulation of atmospheric boundary layers in a wind tunnel. Wind and Structures, 7(2):131144, 2004.

[116] P. Sagaut. Large Eddy Simulation for Incompressible Flows: An Introduction. Scientific Computation. Springer-Verlag, Berlin Heidelberg, 3 edition, 2006.

[117] A. Leonard. Energy Cascade in Large-Eddy Simulations of Turbulent Fluid Flows. In F. N. Frenkiel and R. E. Munn, editors, Advances in Geophysics, 
volume 18 of Turbulent Diffusion in Environmental Pollution, pages 237-248. Elsevier, January 1975.

[118] P. R. Spalart and S. Allmaras. A one-equation turbulence model for aerodynamic flows. Reno, N.V., 1992. American Institute of Aeronautics and Astronautics.

[119] D. Anderson, J. C. Tannehill, and R. H. Pletcher. Computational Fluid Mechanics and Heat Transfer. CRC Press, Boca Raton, 3rd edition edition, April 2011.

[120] B. E. Launder, A. Morse, W. Rodi, and D. B. Spalding. Prediction of free shear flows: A comparison of the performance of six turbulence models. page 66 . NASA, January 1973.

[121] W. Rodi. The prediction of free turbulent boundary layers by use of a twoequation model of turbulence. PhD thesis, Imperial College London (University of London), London, 1973.

[122] D. C. Wilcox. Turbulence Modeling for CFD. D C W Industries, La Cãnada, Calif, 2nd edition edition, July 1998.

[123] P. Doron, L. Bertuccioli, J. Katz, and T. R. Osborn. Turbulence Characteristics and Dissipation Estimates in the Coastal Ocean Bottom Boundary Layer from PIV Data. Journal of Physical Oceanography, 31(8):2108-2134, August 2001.

[124] K. R. Sreenivasan. On the universality of the Kolmogorov constant. Physics of Fluids, 7(11):2778-2784, November 1995.

[125] J. de Jong, L. Cao, S. H. Woodward, J. P. L. C. Salazar, L. R. Collins, and H. Meng. Dissipation rate estimation from PIV in zero-mean isotropic turbulence. Experiments in Fluids, 46(3):499, October 2008.

[126] A. E. Perry and B. D. Fairlie. Critical Points in Flow Patterns. In F. N. Frenkiel and R. E. Munn, editors, Advances in Geophysics, volume 18 of International Union of Theoretical and Applied Mechanics and International Union of Geodesy and Geophysics, pages 299-315. Elsevier, January 1975.

[127] A. Davey. Boundary-layer flow at a saddle point of attachment. Journal of Fluid Mechanics, 10(4):593-610, June 1961. 
[128] G. Scheuermann, H. Hagen, H. Kruger, and A. Rockwood. Visualizing Critical Points of Arbitrary Poincaré -Index. In Scientific Visualization Conference (dagstuhl '97), pages 277-277, June 1997.

[129] G. Scheuermann. Topological Vector Field Visualization with Clifford Algebra. In H. Fiedler, O. Günther, W. Grass, S. Hölldobler, G. Hotz, R. Reischuk, B. Seeger, and D. Wagner, editors, Ausgezeichnete Informatikdissertationen 1999, GI-Dissertationspreis, pages 213-222. Vieweg+Teubner Verlag, Wiesbaden, 2000.

[130] K. Gjøystdal. Classifying zeros in three dimensional vector fields. Master's thesis, The Norwegian University of Science and Technology, Trondheim, Norway, 2004.

[131] J. M. Greene. Vortex Nulls and Magnetic Nulls. In Topological Fluid Mechanics, pages 478-484. Cambridge University Press, Cambridge, UK, 1990.

[132] J. M. Greene. Locating three-dimensional roots by a bisection method. Journal of Computational Physics, 98(2):194-198, February 1992.

[133] M. S. Chong, A. E. Perry, and B. J. Cantwell. A general classification of threedimensional flow fields. Physics of Fluids A: Fluid Dynamics, 2(5):765-777, May 1990.

[134] J. W. Reyn. Classification and description of the singular points of a system of three linear differential equations. Zeitschrift für angewandte Mathematik und Physik ZAMP, 15(5):540-557, September 1964.

[135] K. Furuheim and M. F. Aasen. Classification and Visualization of Critical Points in 3D Vector Fields. Master's thesis, University of Oslo, Oslo, Norway, 2008.

[136] J. Zhou, R. J. Adrian, S. Balachandar, and T. M. Kendall. Mechanisms for generating coherent packets of hairpin vortices in channel flow. Journal of Fluid Mechanics, 387:353-396, May 1999. Publisher: Cambridge University Press.

[137] R. J. Adrian, K. T. Christensen, and Z.-C. Liu. Analysis and interpretation of instantaneous turbulent velocity fields. Experiments in Fluids, 29(3):275-290, September 2000.

[138] J. C. R. Hunt, A. A. Wray, and P. Moin. Eddies, streams, and convergence zones in turbulent flows. In Proceedings of the Summer Program 1988, December 1988. 
[139] J. L. Lumley. Similarity and the Turbulent Energy Spectrum. The Physics of Fluids, 10(4):855-858, April 1967.

[140] L. Sirovich. Turbulence and the Dynamics of Coherent Structures. Part I. Coherent Structures. Quarterly of Applied Mathematics, 45(3):561-571, 1987.

[141] G. Berkooz, P. Holmes, and J. L. Lumley. The proper orthogonal decomposition in the analysis of turbulent flows. Annual Review of Fluid Mechanics, 25:539-575, 1993.

[142] S. Bernero and H. E. Fiedler. Application of particle image velocimetry and proper orthogonal decomposition to the study of a jet in a counterflow. Experiments in Fluids, 29(1):S274-S281, December 2000.

[143] L. Graftieaux, M. Michard, and N. Grosjean. Combining PIV, POD and vortex identification algorithms for the study of unsteady turbulent swirling flows. Measurement Science and Technology, 12(9):1422-1429, August 2001.

[144] J. Pedersen and K. Meyer. POD analysis of flow structures in a scale model of a ventilated room. Experiments in Fluids, 33(6):940-949, December 2002.

[145] K. E. Meyer, J. M. Pedersen, and O. Özcan. A turbulent jet in crossflow analysed with proper orthogonal decomposition. Journal of Fluid Mechanics, 583:199-227, July 2007.

[146] B. Wieneke. PIV uncertainty quantification from correlation statistics. Measurement Science and Technology, 26(7):074002, June 2015.

[147] J.-S. Wu and G. M. Faeth. Effect of ambient turbulence intensity on sphere wakes at intermediate Reynolds number. AIAA Journal, 33(1):171-173, 1995.

[148] D. Legendre, A. Merle, and J. Magnaudet. Wake of a spherical bubble or a solid sphere set fixed in a turbulent environment. Physics of Fluids, 18(4):048102, April 2006.

[149] I. Eames, C. Jonsson, and P. B. Johnson. The growth of a cylinder wake in turbulent flow. Journal of Turbulence, 12:N39, January 2011.

[150] F. Diaz, J. Gavalda, J. G. Kawall, J. F. Keffer, and F. Giralt. Asymmetrical wake generated by a spinning cylinder. AIAA Journal, 23(1):49-54, 1985.

[151] H. Akima. A New Method of Interpolation and Smooth Curve Fitting Based on Local Procedures. Journal of the ACM, 17(4):589-602, October 1970. 
[152] S. J. Beresh, N. E. Miller, and B. Smith. Practical Challenges in the Calculation of Turbulent Viscosity from PIV Data. In 2018 Aerodynamic Measurement Technology and Ground Testing Conference, Atlanta, Georgia, June 2018. American Institute of Aeronautics and Astronautics.

[153] H. Akima. A Method of Bivariate Interpolation and Smooth Surface Fitting for Values Given at Irregularly Distributed Points. 1975.

[154] G. A. Freedland, G. Eliason, S. A. Solovitz, and R. B. Cal. The role of turbulent inflow on the development of a round jet in cross-flow. International Journal of Heat and Fluid Flow, 84:108592, August 2020.

[155] P. Saarenrinne and M. Piirto. Turbulent kinetic energy dissipation rate estimation from PIV velocity vector fields. Experiments in Fluids, 29(1):S300-S307, December 2000.

[156] N. E. Miller, S. J. Beresh, and M. F. Barone. Direct Calculation of RANS Model Parameters from Jet-in-Crossflow PIV Data. In AIAA Scitech 2019 Forum. American Institute of Aeronautics and Astronautics, 2019.

[157] J. S. McNeal, G. Freedland, L. G. Mastin, R. B. Cal, and S. A. Solovitz. Investigating the Accuracy of One-Dimensional Volcanic Plume Models using Laboratory Experiments and Field Data. Journal of Geophysical Research: Solid Earth, 124(11):11290-11304, 2019.

[158] H Riedel and M Sitzmann. In-flight investigations of atmospheric turbulence. Aerospace Science and Technology, 2(5):301-319, July 1998. 


\section{Appendix A}

\section{Shear Layer Identification}

Isolation of the leading edge and lee-side shear layer edge is accomplished by using two components, a) the square of the shear stress component, $\left\langle u^{\prime} v^{\prime}\right\rangle_{r}^{2}$, and b) the square of the vorticity, $\omega_{z, r}=\left\langle\partial_{r_{*}} V_{r}-\partial_{\xi_{*}} U_{r}\right\rangle^{2}$. These components produced two distinct peaks that separate the two sides of the jet, as seen in figure 4.8 in Chapter 4 and figure A.1 below.

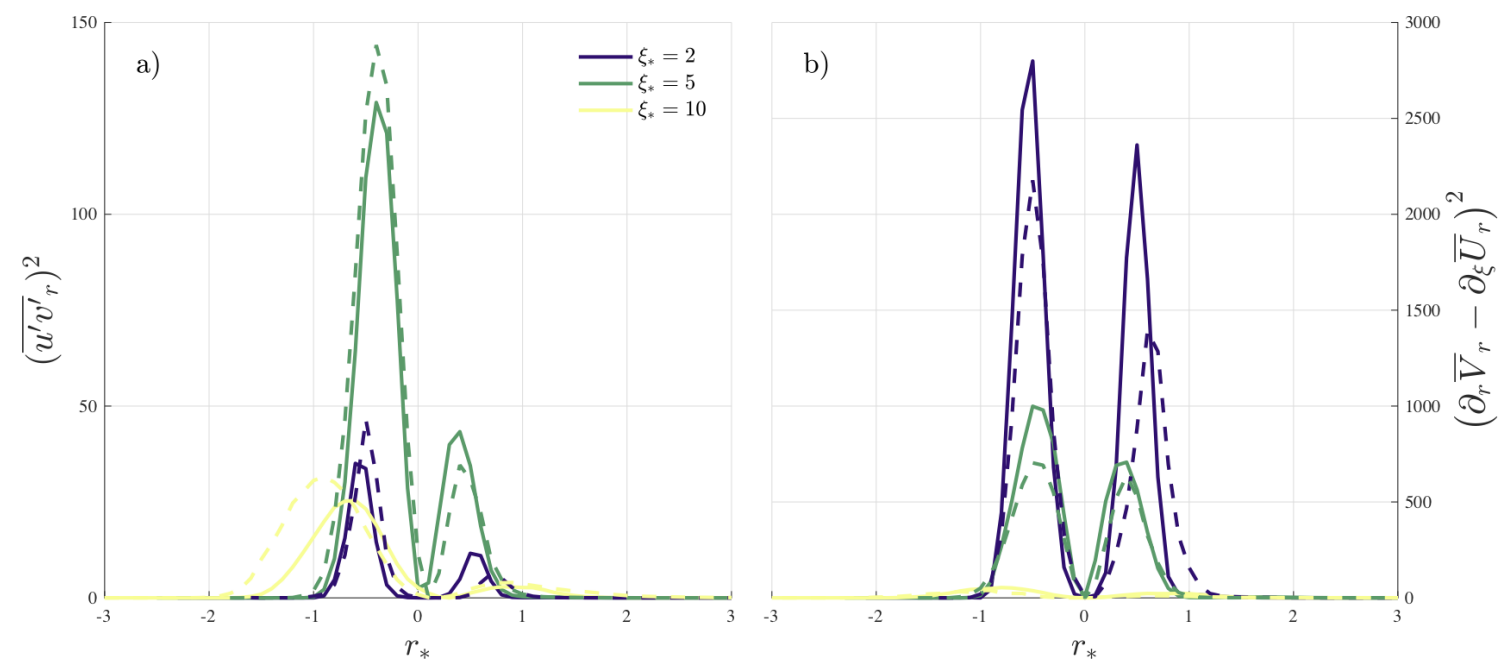

Figure A.1: Jet profiles for the squared a) Reynolds shear stress, $\left\langle u^{\prime} v^{\prime}\right\rangle_{r}^{2}$, and b) vorticity, $\left\langle\partial_{r_{*}} \bar{V}_{R}-\partial_{\xi_{*}} \bar{U}_{r}\right\rangle^{2}$ for one $\mathrm{PG}$ and one AG case at three locations.

To evaluate the width of the jet, these profiles are separated into leading edge and lee-side peaks. The curves are fitted with a Gaussian normal distribution of the 
form

$$
f\left(r_{*}\right)=\frac{1}{\sigma \sqrt{2 \pi}} \exp \left(\frac{-\left(r_{*}-\mu\right)^{2}}{2 \sigma^{2}}\right)
$$

where $f\left(r_{*}\right)$ is either the profile chosen, $r_{*}$ is the radial location, $\sigma$ is the standard deviation and $\mu$ is the location of the maxima. To ensure the validity of the results, a minimum coefficient of determination was set as $R^{2} \geq 0.95$.

From the coefficients output, the edges of the shear layer were defined by evaluating the full width at tenth maximum, defined as

$$
\mathrm{FWTM}=2 \sigma \sqrt{2 \ln 10},
$$

allowing the shear layer to be evaluated at each height as

$$
R_{L E}=\mu_{L E}-\sigma \sqrt{2 \ln 10} \quad R_{L S}=\mu_{L S}+\sigma \sqrt{2 \ln 10}
$$

For each location along the jet, the leading edge and lee-side are evaluated so as to provide a raw boundary for the data. This includes capturing stray points that would disrupt additional computations. Therefore, additional filtering is required. The radial locations are averaged over $1 D$ increments and fit with a smoothing spline. The fitted data is then compared to the raw data with the coefficient of determination restricted to $R^{2} \geq 0.99$. The resulting figure (A.2) illustrates the shear layer boundaries. 


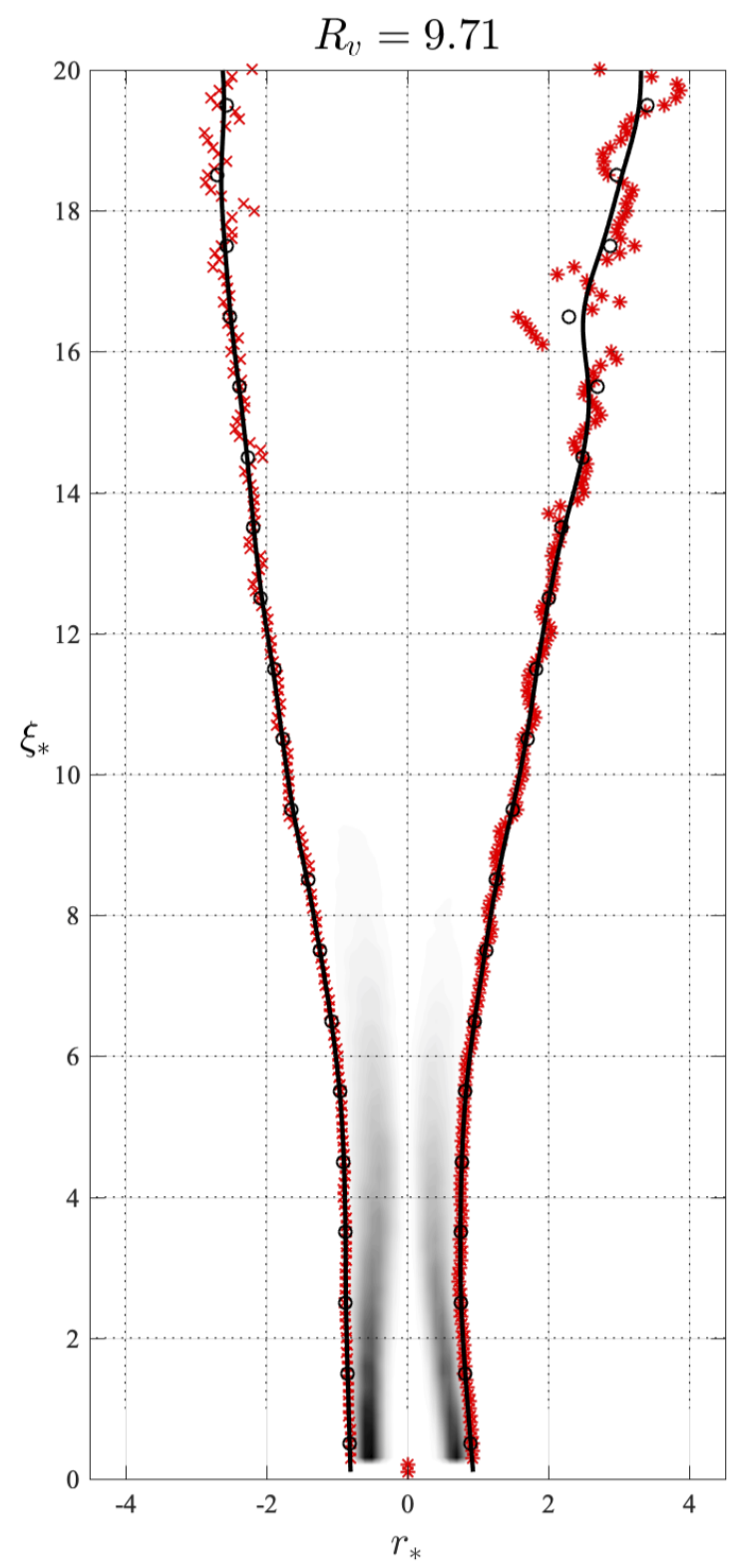

Figure A.2: Shear layer evaluation and filtering for PG case $R_{v}=9.71$. 


\section{Appendix B}

\section{Relative Axis Transformations}

For a bending JICF, a consistent boundary definition is required to compare transport across various $R_{v}$ and inflow conditions. For each case, the trajectory of the jet will vary, and so by considering each flow relative to this path, the behavior can be compared through identification of common features.

The trajectory is found by evaluating the mean velocity component $\bar{V}_{*}$ and isolating the maximum at each height. The data is then smoothed by fitting with a power law of the form $y=A x^{b}$, which also provides a smoothed slope. At each location along the trajectory, the inverse of the slope is used to extend a normal line both up- and downstream of the centerline. This normal line, defined as the radial distance off the centerline, $r_{*}$, is spaced at increments of $r_{*}=0.1$. All velocity components are interpolated using a bivariate spline interpolation method [151]. Using the centerline angle relative to the cross-flow, defined as $\theta_{C L}$, the velocity components are transformed to deliniate the velocity normal to the centerline, $U_{r}$, and tangential to the centerline, $V_{r}$, using the matrix transform $x_{j}^{\prime}=Q_{i j} \cdot x_{j}$ for a 
three dimensional coordinate system,

$$
\left[\begin{array}{c}
\bar{U}_{r} \\
\bar{V}_{r} \\
\bar{W}_{r}
\end{array}\right]=\left[\begin{array}{ccc}
\cos \theta_{C L} & -\sin \theta_{C L} & 0 \\
\sin \theta_{C L} & \cos \theta_{C L} & 0 \\
0 & 0 & 1
\end{array}\right]\left[\begin{array}{c}
\bar{U} \\
\bar{V} \\
\bar{W}
\end{array}\right] .
$$

In figure 2.1 (section 2.1), $r_{*}$ and $\xi_{*}$ are the distances normal to the centerline and the jet length, respectively, non-dimensionalized by the diameter of the jet. The resultant transform "unbends" the jet as shown in the right panel of figure 2.1. 


\section{Appendix C}

\section{Plumeria Conic Area Extraction}

From the Plumeria outputs, the radius of the jet is defined at three points, the leading edge $\left(r_{L E}\right)$, the centerline $\left(r_{C L}\right)$ and the lee-side $\left(r_{L S}\right)$. The points are extracted by the corresponding value at each height from $y_{*}=0 \rightarrow 6$ and arranged to form two half ellipses that follow the form

$$
1=\frac{x^{2}}{a^{2}}+\frac{y^{2}}{b^{2}} .
$$

The spatial coordinates are transformed into cylindrical coordinates,

$$
1=\frac{[r \cos (\theta)]^{2}}{a^{2}}+\frac{[r \sin (\theta)]^{2}}{b^{2}},
$$

and reorganized to solve for $r$ gives us the final form,

$$
r(\theta)=\sqrt{\frac{a^{2} b^{2}}{a^{2} \sin ^{2}(\theta)+b^{2} \cos ^{2}(\theta)}}
$$




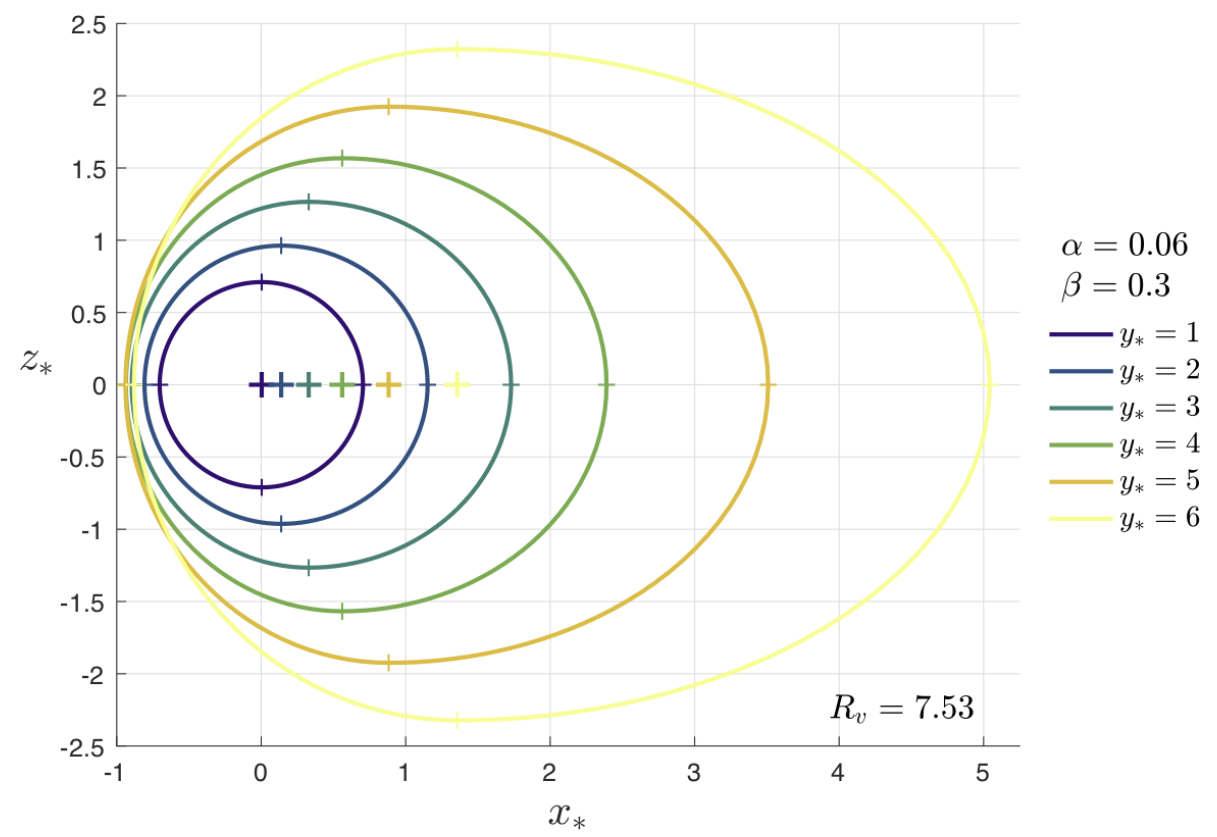

Figure C.1: Computed plume cross-sectional area for one case $(\alpha=0.06, \beta=$ $\left.0.3, R_{v}=7.53\right)$. These profiles will be superimposed over the PIV planes to determine the ideal coefficients for predicting plume expansion.

where

$$
\begin{aligned}
& a=r_{L E}, b=r_{C L} \quad \text { for }-\pi / 2 \leq \theta<\pi / 2 \\
& a=r_{L S}, b=r_{C L} \quad \text { for } \pi / 2 \leq \theta<3 \pi / 2
\end{aligned}
$$

The radius is then transformed back onto cartesian coordinates. The output of this transformations can be seen in figure C.1. For analysis, these will then be superimposed on the velocity fields to identify the optimal settings for $\alpha$ and $\beta$, as well as identifying any unique behaviors that would significantly impact future models.

Additionally, these plumes can be reconstructed to evaluate the $3 \mathrm{D}$ volume of 
the plume (figure C.2).

The reconstructions of the jet, shown in figure 4.19, are compared to the Plumeria data by evaluating the total volume of the jet and the change in area between heights. 


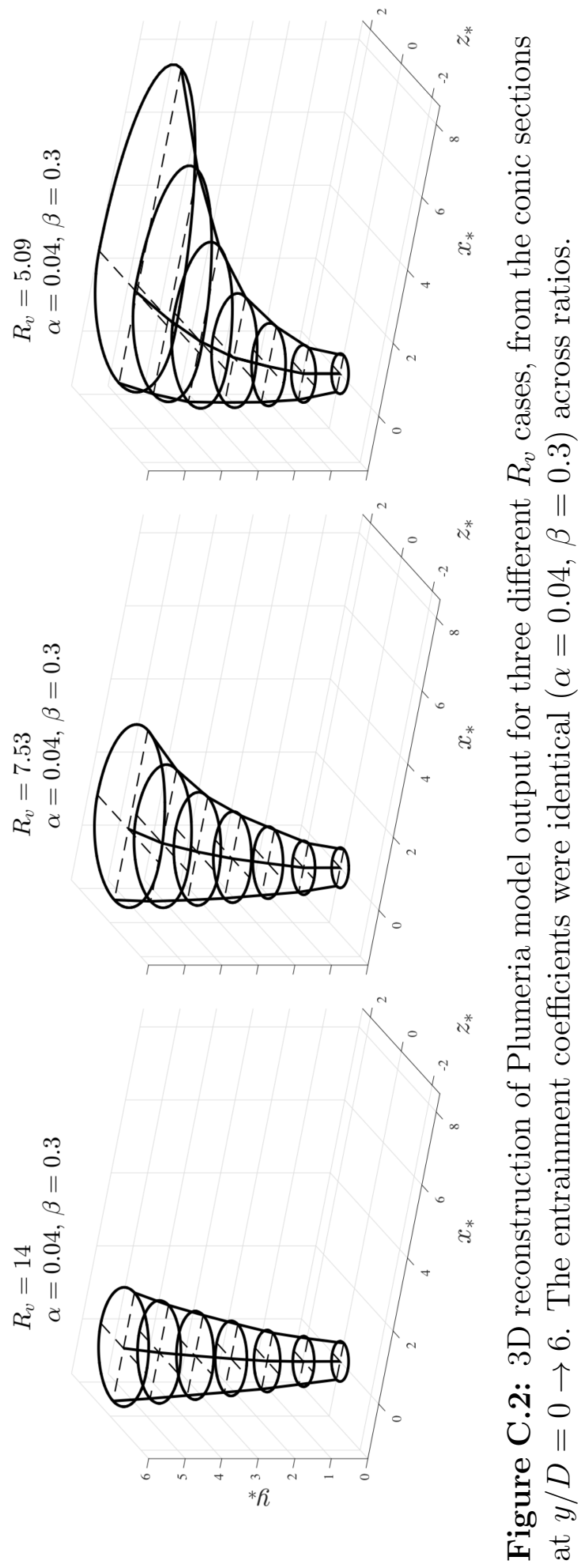

